\title{
CEsifo \\ WORKING

\section{A Residual-based Threshold Method for Detection of Units that are Too Big to Fail in Large Factor Models}

G. Kapetanios, M. H. Pesaran, S. Reese 


\section{Impressum:}

CESifo Working Papers

ISSN 2364-1428 (electronic version)

Publisher and distributor: Munich Society for the Promotion of Economic Research - CESifo $\mathrm{GmbH}$

The international platform of Ludwigs-Maximilians University's Center for Economic Studies and the ifo Institute

Poschingerstr. 5, 81679 Munich, Germany

Telephone +49 (o)89 2180-2740, Telefax +49 (o)89 2180-17845, email office@cesifo.de

Editors: Clemens Fuest, Oliver Falck, Jasmin Gröschl

www.cesifo-group.org/wp

An electronic version of the paper may be downloaded

- from the SSRN website: $\quad$ www.SSRN.com

- from the RePEc website: $\quad$ www.RePEc.org

- from the CESifo website: www.CESifo-group.org/wp 


\title{
A Residual-based Threshold Method for Detection of Units that are Too Big to Fail in Large Factor Models
}

\begin{abstract}
The importance of units with pervasive impacts on a large number of other units in a network has become increasingly recognized in the literature. In this paper we propose a new method to detect such influential or dominant units by basing our analysis on unit-specific residual error variances in the context of a standard factor model, subject to suitable adjustments due to multiple testing. Our proposed method allows us to estimate and identify the dominant units without the a priori knowledge of the interconnections amongst the units, or using a short list of potential dominant units. It is applicable even if the cross section dimension exceeds the time dimension, and most importantly it could end up with none of the units selected as dominant when this is in fact the case. The sequential multiple testing procedure proposed exhibits satisfactory small-sample performance in Monte Carlo simulations and compares well relative to existing approaches. We apply the proposed detection method to sectoral indices of US industrial production, US house price changes by states, and the rates of change of real GDP and real equity prices across the world's largest economies.
\end{abstract}

JEL-Codes: C180, C230, C550.

Keywords: dominant units, factor models, systemic risk, cross-sectional dependence, networks.

\author{
George Kapetanios \\ King's College, London \\ London / United Kingdom \\ george.kapetanios@kcl.ac.uk
}

\author{
M. Hashem Pesaran \\ University of Southern California \\ Los Angeles / CA / USA \\ pesaran@usc.edu
}

\author{
Simon Reese \\ USC Dornsife INET \\ Los Angeles / CA / USA \\ sreese@usc.edu
}

November 29, 2018

We are grateful to Natalia Bailey, Alexander Chudik, Ron Smith, Vanessa Smith, Donggyu Sul, Takashi Yamagata and Cynthia Yang for constructive comments on a preliminary version of the paper. 


\section{Introduction}

Detecting the presence of economic agents, whose behavior influences a large number of peers, has become a relevant issue in several areas of economics. For example, in banking and finance it is of interest to consider formal ways of identifying whether particular financial institutions are dominant and present systemic risks. At times of economic and financial crises it is often of interest to know if a certain corporation, particularly among financial institutions, is so large and interconnected that their failure could lead to cascade effects with associated systemic risk for the economy as a whole. Such units are often referred to as 'too big to fail' and their existence is debated in the press and in public policy forums, although empirical evidence in support of such claims is often lacking. In cases where information on the interconnections across the units exist, it is possible to use such information to detect the most influential unit in the network and examine its degree of dominance. An important example is inputoutput data used to analyse the role that individual production units, such as industrial sectors, play in propagating shocks across the economy. A major recent contribution in this area is by Acemoglu, Carvalho, Ozdaglar, and Tahbaz-Salehi (2012) who suggest using the shape parameter of a power law assumed for the degree sequence of a network to measure the extent to which variations in aggregate volatility are affected by shocks to individual units within the network. Further developments are provided by Acemoglu, Akcigit, and Kerr (2016) and Acemoglu, Autor, Dorn, Hanson, and Price (2016). In related work, Pesaran and Yang (2016) propose extremum estimators based on outdegrees of a network to detect and identify the identity of the dominant units in the network and to estimate their degrees of pervasiveness.

In cases where information on network connections is not available, it is still possible to identify the dominant units in a network, if there is a sufficient number of time series observations $(T)$ on all the units $(N)$ in the network. In this paper we suppose that such time series observations are available and address the problem of jointly determining the number as well as the identity of units in the network that are dominant, in the sense that they influence almost all other units in the network. The central hub in a Star network provides a simple example of a dominant unit. We identify dominant units by considering statistical significance of the correlations across the units once possible effects of external influences on the network have been filtered out. The presence of dominant units is also closely related to the notion of 'too big to fail' often used in the context of financial and production networks. However, it is important to bear in mind that the two concepts are not identical. For example, a unit that is too big to fail may become influential mainly in crisis periods, implying a nonlinear behaviour that our linear model may not be best equipped to handle.

Our approach shares some features with previous contributions on the same subject (see e.g. Bai and Ng, 2006; Parker and Sul, 2016; Brownlees and Mesters, 2018) but significantly improves on existing research in a number of respects. First, we allow for the possibility that the network under consideration does not include any dominant unit in the first place. This is a leading case of interest and, in fact, some of our empirical work confirms its practical importance. Secondly, we do not require a priori information on a potential list of dominant units or observations on network linkages. This is a key advantage relative to contributions in the production network literature which relies on the availability of input-output tables. Third, our detection procedure can determine dominant units from a large number of potential candidates, even in the presence of external common factors that could potentially influence all the units (including the dominant units) in the network. Finally, our procedure applies even if 
$N>T$, which is an important consideration in practice where in many applications of interest the number of time series observations is limited either because of unavailability of data or due to structural breaks.

A major implication of the existence of dominant units is that the data can be represented by a factor model where variation in the dominant units is perfectly explained by the true factors. This view on dominant units reflects the fact that an influential unit can be viewed as a common factor for all other units in the network. Consequently, factor estimates obtained from the dataset will have close to perfect explanatory power for true dominant units. Using this result, we consider the residual variance from regressions of individual units on the factor estimates as a metric that quantifies the explanatory ability of the estimated factors. Based on ideas from multiple testing we then construct thresholds that determine whether the residual variance estimated for a given unit is sufficiently small to identify that unit as dominant. We find that thresholding residual variances across the units provides a powerful approach with a number of desirable characteristics and good small sample performance.

A further defining characteristic of our work is to consider refinements that again make use of multiple testing to allow for the possibility that identified dominant units may not be fully pervasive - that is they may only affect a subset of cross-sectional units. This further distinguishes our work from existing methods which either do not pay much attention to such weak cross-sectional dependence structures or are unclear about the motivation and nature of these structures. The use of multiple testing focuses on the possibility that some units selected as dominant might only affect a majority of the units in the network rather than being fully pervasive with non-zero effects on all units. We feel that local to zero representations of factor loadings, which are sometimes used in the literature, where the size of the loadings depend on the sample size and tend to zero as this size rises, are less persuasive as a model for economic networks than the weak dependence formulation that we consider in this paper.

Monte Carlo simulations suggest that our refined thresholding method performs very well in finite-sample, and most importantly, it reliably detects the absence of dominant units from a dataset with many potential candidates. Furthermore, if influential cross section units are part of the model specification, our detection methodology succeeds in jointly detecting their total number and their identities. The proposed method also works well even if $N$ is much larger than $T$, and unlike other methods proposed in the literature, its false discovery rate is very low and tends to zero as $N$ and $T \rightarrow \infty$.

The proposed detection procedure is applied to sectoral indices of U.S. industrial production (already investigated in the literature), as well as to the rates of change of real GDP and real equity prices across the world's largest economies over the period 1979Q2-2016Q4. Unlike other detection methods proposed in the literature, we do not find convincing evidence that there are dominant sectors within the U.S. industrial production, or that there exist dominant economies or equity markets in the global system, once we adequately allow for the presence of common factors. Finally, we apply the new method to real U.S. house price changes across the 48 mainland states, and find evidence that New York is dominant, in contrast to the other methods that select states such as New Hampshire, Nevada, North Carolina, Maryland and Virginia (just to mention a few) and not New York as dominant.

The paper is structured as follows. Section 2 presents a review of the existing literature. Section 3 provides the main setup of our approach and details our theoretical results. Further refinements are discussed in Section 5. Sections 6 and 7 present simulation and empirical evidence on the relative performance of our method compared to existing ones. Formal proofs 
and additional simulation results are relegated to Appendix A and an online supplement, respectively.

Notation: Generic positive finite constants are denoted by $C$ when large, and $c$ when small. They can take different values at different instances. $\rightarrow^{p}$ denotes convergence in probability as $N, T \rightarrow \infty . \lambda_{\max }(\boldsymbol{A})$ and $\lambda_{\min }(\boldsymbol{A})$ denote the maximum and minimum eigenvalues of matrix A. $\boldsymbol{A}>0$ denotes that $\boldsymbol{A}$ is a positive definite matrix. $\|\boldsymbol{A}\|$ and $\|\boldsymbol{A}\|_{F}$ denote the spectral and Frobenius norm of matrix $\boldsymbol{A}$. If $\left\{f_{n}\right\}_{n=1}^{\infty}$ is any real sequence and $\left\{g_{n}\right\}_{n=1}^{\infty}$ is a sequences of positive real numbers, then $f_{n}=O\left(g_{n}\right)$, if there exists $C$ such that $\left|f_{n}\right| / g_{n} \leq C$ for all $n$. $f_{n}=o\left(g_{n}\right)$ if $f_{n} / g_{n} \rightarrow 0$ as $n \rightarrow \infty$. If $\left\{f_{n}\right\}_{n=1}^{\infty}$ and $\left\{g_{n}\right\}_{n=1}^{\infty}$ are both positive sequences of real numbers, then $f_{n}=\ominus\left(g_{n}\right)$ if there exists $n_{0} \geq 1$ and positive finite constants $C_{0}$ and $C_{1}$, such that $\inf _{n \geq n_{0}}\left(f_{n} / g_{n}\right) \geq C_{0}$, and $\sup _{n \geq n_{0}}\left(f_{n} / g_{n}\right) \leq C_{1}$.

\section{Related literature}

Asset pricing models have motivated the earliest approaches aimed at determining whether a given set of observed time series coincides with one of the estimated common factors (principal components) from a large panel dataset. Bai and Ng (2006) regress each observed candidate series onto the estimated factors and propose statistics to test the equality between the model fit from the aforementioned regression and the observed values of a list of (assumed) potential influential variables. The framework considered by these authors is one where economic theory reduces the number of potential influential variables to a small, fixed number of economic indicators that are not part of the large dataset at hand. Consequently, using their framework to identify dominant units in large datasets without any means of reducing the number of candidates is problematic. This pitfall was recognized by Parker and Sul (2016) who provide an alternative approach to that suggested by Bai and $\mathrm{Ng}$ (2006) and consider the identification of dominant units in a large dataset as a special case. ${ }^{1}$ Parker and Sul focus on the idiosyncratic components of the estimated factor model and identify an observed series as a dominant unit if it can replace at least one of the estimated factors in the factor model without introducing common factors in the idiosyncratic components. ${ }^{2}$ In order to address multiple testing concerns, a rule of thumb is suggested to restrict the number of potential dominant units. However, this only mitigates the problem rather than providing a full solution. A more general solution is provided by Brownlees and Mesters (2018), who use the sample concentration matrix of all the units in the network to identify as dominant units those units whose concentration matrix column norms are considerably larger than those of the remaining units. ${ }^{3}$ Under certain regularity conditions, Brownlees and Mesters show that their procedure consistently partitions the units into dominant and non-dominant by ordering column norms in descending order and choosing the maximum ratio between two successive, ordered column norms.

While suggesting a consistent detection procedure without needing to estimate the common factors in the data, Brownlees and Mesters (2018) require the number of time periods to be larger than the number of cross-section units $(T>N)$, and assume that there exists at least

\footnotetext{
${ }^{1}$ In Parker and Sul (2016) a dominant unit is referred to as the dominant leader.

${ }^{2}$ Further empirical applications of the Parker and Sul method (in a simpler form) are provided by Gaibulloev, Sandler, and Sul (2013) and Greenaway-McGrevy, Mark, Sul, and Wu (2018). Soofi-Siavash (2018) also considers a version of the Parker and Sul method which is applicable to any cross-section unit taken as potentially dominant, and provides an application to the industrial sectors in the U.S..

${ }^{3}$ Brownlees and Mesters (2018) employ the term granular unit to denote a dominant unit.
} 
one dominant unit in the network. These two requirements result in considerable restrictions in empirical practice. First, many datasets, notably those involving aggregate economic indicators, have a number of cross section units that is approximately as large as the number of time periods, if not larger. Even if the time dimension of the dataset is sufficiently large, sub-samples of interest (due to structural breaks) might be too short to allow for a separate investigation. Second, it is crucial to allow for the possibility that none of the units in the network is particularly influential. The relevance of this case is given by recent contributions that track the effect of sector-specific shocks on aggregate fluctuations. For example, application of a structural model to data on U.S. industrial production leads Foerster, Sarte, and Watson (2011, p.21) to conclude that "[...] linkages alone and uncorrelated sector-specific shocks implies noticeably less co-movement across sectors than in U.S. data." Further evidence is given in Pesaran and Yang (2016) who develop an estimator for the degree of dominance of the most pervasive unit in a network. Their application on U.S. input-output tables reveals that there is "[...] some evidence of sector-specific shock propagation, but [that] such effects do not seem sufficiently strong and long-lasting [...]" in the sense that the aggregate effect of sectoral shocks vanishes as the number of sectors in the economy increases. Finally, while the two studies cited above allow for the absence of dominant units, they crucially rely on the availability of input-output matrices as a measure of linkages between cross-sections. Comparable information may not always be available, thus making it impossible to use the techniques in these studies. By contrast, the approach proposed in the current paper is applicable to any large dimensional panels without requiring the presence of a minimum number of dominant units in the panel.

\section{Panel data models with dominant units}

Suppose $T$ time series observations are available on $N$ cross section units denoted by $x_{i t}$, for $i=1,2, \ldots, N$ and $t=1,2, \ldots, T$. We are interested in determining the number and identity of dominant or pervasive units (if any), in this panel. To define the concept of a dominant unit, we proceed by specifying that all cross section units can be modeled using unobserved common factors. More formally, we consider the following data generating process (DGP)

$$
\begin{aligned}
\mathbf{x}_{a t} & =\mathbf{A}_{h} \mathbf{h}_{t}+\mathbf{A}_{g} \mathbf{g}_{t}, \\
\mathbf{x}_{b t} & =\mathbf{B}_{a} \mathbf{x}_{a t}+\mathbf{B}_{g} \mathbf{g}_{t}+\mathbf{u}_{t},
\end{aligned}
$$

for $t=1,2, \ldots, T$, where $\mathbf{x}_{a t}$ and $\mathbf{x}_{b t}$ are $m \times 1$ and $n \times 1$ vectors of observations at time $t$ on the dominant and non-dominant units, respectively. Thus $N=m+n$.

The $m$ dominant units, $x_{a, j t}, j=1,2, \ldots, m$ affect the non-dominant units, $x_{b, i t}, i=m+$ $1, m+2, \ldots, N$ via the $n \times m$ matrix of loading coefficients $\mathbf{B}_{a}=\left(b_{a, i j}\right)$, where $n=N-m$. For $x_{a, j t}$ to be a dominant unit we must have

$$
\sum_{i=m+1}^{N}\left|b_{a, i j}\right|=\ominus(n), j=1,2, \ldots, m
$$

In other words, for a unit to be dominant it must have non-zero effects on almost all other units in the panel or network. Following Chudik, Pesaran, and Tosetti (2011), we could also consider units that are not dominant but still quite influential. Suppose that there exists an ordering 
of the non-dominant units such that unit $x_{a, j t}$ only affects the non-dominant units, $x_{b, i t}$, whose index $i \leq\left\lfloor n^{\alpha_{j}}\right\rfloor$, where $\alpha_{j}\left(0<\alpha_{j} \leq 1\right)$ is an exponent parameter that measures the degree of the dominance of $x_{a, j t}$ in the panel. ${ }^{4}$ This requirement can be written equivalently as

$$
\sum_{i=m+1}^{N}\left|b_{a, i j}\right|=\ominus\left(n^{\alpha_{j}}\right), \text { for } j=1,2, \ldots, m,
$$

which is a natural generalization of (3). The unit $x_{a, j t}$ with $\alpha_{j}<1$ can be viewed as a weak factor, but as argued in Bailey, Kapetanios, and Pesaran (2016) and Bailey, Kapetanios, and Pesaran (2018), for $x_{a, j t}$ to have pervasive effects on other units we need $\alpha_{j}$ to be reasonably close to unity. Clearly, the values of $\alpha_{j} \leq 1 / 2$ can be ruled out since for such values, $x_{a, j t}$ becomes so weak that it loses many of the standard characteristics, associated with factor variables. In practice we might need to focus on exponents that fall in the range $2 / 3<\alpha_{j} \leq 1$ before we can be confident that unit $x_{a, j t}$ has non-negligible impacts on other units in the network. In terms of the general definition (4), for all elements of $\mathbf{x}_{a t}$ to be dominant it is required that $\alpha_{j}=1$ for $j=1,2, \ldots, m$, and dominant units can be regarded as strong factors. While our theory focuses on $\alpha_{j}=1, j=1,2, \ldots, m$, it can be extended to $\alpha_{j} \leq 1$, using ideas in the above cited papers. It is also possible to estimate the exponent $\alpha_{j}$ once the unit $x_{a, j t}$ is selected as dominant/influential. However, such extensions are beyond the scope of the present paper.

The $k \times 1$ vector $\mathbf{g}_{t}$ contains common "external" factors affecting both dominant and nondominant units via the $m \times k$ and the $n \times k$ loading matrix $\mathbf{A}_{g}$ and $\mathbf{B}_{g}$, respectively. The dominant units can also be viewed as "internal" factors. Lastly, the $m \times 1$ vector $\mathbf{h}_{t}$ as well as the $n \times 1$ vector $\mathbf{u}_{t}$ model stochastic variation that originates in the dominant and non-dominant units, respectively. To simplify the exposition we abstract from deterministic effects such as intercepts or linear trends and without loss of generality assume that $x_{i t}$ have zero means and finite variances. Define now the $p \times 1$ vector $\mathbf{f}_{t}=\left(\mathbf{h}_{t}^{\prime}, \mathbf{g}_{t}^{\prime}\right)^{\prime}=\left(f_{1 t}, f_{2 t}, \ldots, f_{p t}\right)^{\prime}$ where $p=m+k .^{5}$ Using this vector, the dominant unit model (1)-(2) can be written as a restricted static factor model, given by

$$
\begin{aligned}
\left(\begin{array}{c}
\mathbf{x}_{a t} \\
\mathbf{x}_{b t}
\end{array}\right) & =\left(\begin{array}{c}
\mathbf{A}_{a} \\
\mathbf{A}_{b}
\end{array}\right) \mathbf{f}_{t}+\left(\begin{array}{c}
\mathbf{0} \\
\mathbf{u}_{t}
\end{array}\right) \\
& =\mathbf{A f}_{t}+\mathbf{v}_{t},
\end{aligned}
$$

where $\mathbf{A}_{a}=\left(\mathbf{A}_{h}, \mathbf{A}_{g}\right)$ and $\mathbf{A}_{b}=\left(\mathbf{B}_{a} \mathbf{A}_{h}, \mathbf{B}_{a} \mathbf{A}_{g}+\mathbf{B}_{g}\right)$. Additionally, denote by $\mathbf{a}_{i}$ the $i$-th row of $\mathbf{A}=\left(\mathbf{A}_{a}^{\prime}, \mathbf{A}_{b}^{\prime}\right)^{\prime}$. Since a dominant unit is de facto a common factor, then $m \leq p$. It is also shown in Chudik, Pesaran, and Tosetti (2011), that $p$ must be a fixed integer to ensure that $\operatorname{Var}\left(x_{i t}\right)$ is bounded in $N$. Accordingly, we assume that $0 \leq m \leq p<p_{\max }$, where $p_{\max }$ is an upper bound on $p$.

We shall also make the following assumptions:

\section{Assumption 1}

\section{1. $\mathbf{f}_{t}$ is a covariance-stationary stochastic process with $E\left(\mathbf{f}_{t} \mathbf{f}_{t}^{\prime}\right)=\mathbf{I}_{p}$.}

\footnotetext{
${ }^{4}\lfloor a\rfloor$ denotes the integer part of $a$.

${ }^{5}$ The magnitude of $m$ relative to $k$ is immaterial as long as both are fixed.
} 
2. There exist sufficiently large positive constants $C_{0}$ and $C_{1}$ and $s_{f}>0$ such that

$$
\sup _{t} \operatorname{Pr}\left(\left|f_{j t}\right|>a\right) \leq C_{0} \exp \left(-C_{1} a^{s_{f}}\right) \text { for each } j=1,2, \ldots, p \text {. }
$$

3. $T^{-1} \sum_{t=1}^{T} \mathbf{f}_{t} \mathbf{f}_{t}^{\prime} \rightarrow^{p} \mathbf{I}_{p}$ and $\frac{1}{T} \sum_{t=1}^{T}\left[\left\|\mathbf{f}_{t}\right\|^{j}-E\left(\left\|\mathbf{f}_{t}\right\|^{j}\right)\right] \rightarrow^{p} \mathbf{0}, j=3,4$.

\section{Assumption 2}

1. $\mathbf{A}_{a}$ and $\mathbf{A}_{b}$ are parameter matrices, the former satisfying Rank $\left(\mathbf{A}_{a}\right)=m \geq 0$.

2. $\inf _{i}\left\|\mathbf{a}_{i}\right\|>c$, and $\sup _{i}\left\|\mathbf{a}_{i}\right\|<C$, and for any $N=n+m$ ( $m$ being a finite integer)

$$
\begin{aligned}
& \lambda_{\max }\left(n^{-1} \sum_{i=m+1}^{N} \mathbf{a}_{i} \mathbf{a}_{i}^{\prime}\right)<C<\infty, \\
& \lambda_{\min }\left(n^{-1} \sum_{i=m+1}^{N} \mathbf{a}_{i} \mathbf{a}_{i}^{\prime}\right)>c>0 .
\end{aligned}
$$

\section{Assumption 3}

1. The $n \times 1$ vector $\mathbf{u}_{t}$ is defined by

$$
\mathbf{u}_{t}=\mathbf{H} \varepsilon_{t}
$$

where

$$
\boldsymbol{\varepsilon}_{t}=\left(\varepsilon_{m+1, t}, \varepsilon_{m+2, t}, \ldots, \varepsilon_{N t}\right)^{\prime} \sim \operatorname{IID}\left(\mathbf{0}, \mathbf{I}_{n}\right),
$$

and $\sup _{i} T^{-1} \sum_{t=1}^{T} \sum_{t^{\prime}=1}^{T}\left|\operatorname{Cov}\left(\varepsilon_{i t}, \varepsilon_{i t^{\prime}}\right)\right|<C<\infty$.

2. There exist sufficiently large positive constants $C_{0}$ and $C_{1}$ and $s_{\varepsilon}>0$ such that

$$
\sup _{i, t} \operatorname{Pr}\left(\left|\varepsilon_{i t}\right|>a\right) \leq C_{0} \exp \left(-C_{1} a^{s_{\varepsilon}}\right) .
$$

3. $\mathbf{H}=\left(h_{i j}\right)$ is an $n \times n$ matrix with fixed coefficients, with bounded row and column sum norms, formally $\|\mathbf{H}\|_{1}=\sup _{j} \sum_{i=1}^{n}\left|h_{i j}\right|<C$, and $\|\mathbf{H}\|_{\infty}=\sup _{i} \sum_{j=1}^{n}\left|h_{i j}\right|<C$. Furthermore, $\lambda_{\min }\left(\mathbf{H H}^{\prime}\right)>c>0$.

Assumption $4 \mathbf{f}_{t}$ and $\varepsilon_{i s}$ are independent for all $i, s, t$.

Remark 1 Most of the above assumptions relate closely to those made in the literature on the large dimensional factor models (see Remark 2 below). Restricting the covariance matrix of $\mathbf{f}_{t}$ to be the identity matrix is an innocuous simplification, since the factors are identified only up to a p-dimensional rotation. However, since the methodology proposed in this article goes beyond estimation in a large-dimensional factor model, some of the assumptions made above are slightly stronger than those made in the literature. Covariance stationarity of the common factors is one such restriction but does not rule out conditional heteroskedasticity. Our use of results from the multiple testing literature assumes that the probability distributions of $\varepsilon_{i t}$ and $f_{i t}$ have exponentially decaying tails. While this assumption is standard in high-dimensional 
statistics, it implies that all moments of $\varepsilon_{i t}$ and $f_{i t}$ exist and thus sharpens our assumptions beyond those required for the estimation of unobserved factors. This assumption simplifies the theoretical analysis. It can be relaxed, considerably in the case of $f_{i t}$, and replaced with moment assumptions, at the cost of more complex proofs. We choose to avoid this complexity as we are mainly focused on suggesting and analyzing a new methodology. Furthermore, to establish consistency of our proposed criterion, we assume $\varepsilon_{i t}$ to be independently distributed across $i$ and $t .{ }^{6}$ Still, dependence between the elements of the unit-specific component $\mathbf{u}_{t}$ is allowed for by Assumption 3 which admits weak cross-section correlation. The rank condition on $\mathbf{A}_{a}$ in Assumption 2 ensures that $m$ is identified and that $\mathbf{x}_{a t}$ is dominant. Assumption 2 implies strong factors in the sense that the fraction of cross-section units affected is asymptotically nonnegligible. This is a standard property of latent factors in the related literature, as is Assumption 4. On this see, for example, Assumptions L and LFE in Bai and $\mathrm{Ng}$ (2008).

Remark 2 A further consideration concerns how the above assumptions relate to those of the standard factor model literature as set out, for example, in Bai (2003). As noted above our assumptions are stricter, and therefore imply the assumptions made by Bai (2003). In particular, Assumption 1 implies Assumption A of Bai (2003), Assumption 2 implies Assumption B of Bai (2003) and Assumptions 3 and 4 imply Assumptions C, D, E and F1-F2 of Bai (2003) while we note that we have no need for Assumptions F3-F4 of Bai (2003).

As shown in the next section, it is possible to consistently estimate the parameters of the static factor model (5), even if the variance matrix of the $N \times 1$ vector $\mathbf{v}_{t}=\left(\mathbf{0}_{m \times 1}^{\prime}, \mathbf{u}_{t}^{\prime}\right)^{\prime}$, containing the idiosyncratic errors is singular when $m>0$. In our theoretical derivations and Monte Carlo simulations we only require that $p_{\max } \geq p$ is known and base our analysis on $p_{\max }$ principal components of $x_{i t}$ for $i=1,2, \ldots, N$ and $t=1,2, \ldots, T$.

\section{Identification of dominant units via thresholding of error variances}

The idea behind our detection procedure is simple. It exploits the fact that there is a clear separation between the fit of dominant and non-dominant units in terms of the factors, $\mathbf{f}_{t}$ for sufficiently large sample sizes. The fit is expected to be perfect for the dominant units but not for the non-dominant units, as $N$ and $T \rightarrow \infty$. With this in mind we first extract the first $p_{\max }$ principle components (PC) of the observations $x_{i t}$ for $i=1,2, \ldots, N ; t=1,2, \ldots, T$. We then compute the residual sum of squares from the regressions of $x_{i t}$, for $i=1,2, \ldots, N$ on $\hat{\mathbf{f}}_{t}$, where $\hat{\mathbf{f}}_{t}$ is the PC estimator of $\mathbf{f}_{t}$ with $p=p_{\max }$. Specifically, we compute

$$
\hat{\sigma}_{i T}^{2}=\frac{\mathbf{x}_{i}^{\prime} \mathbf{M}_{\hat{\mathbf{F}}} \mathbf{x}_{i}}{T}, \text { for } i=1,2, \ldots, N
$$

where $\mathbf{x}_{i}=\left(x_{i 1}, x_{i 2}, \ldots, x_{i T}\right)^{\prime}$

$$
\mathbf{M}_{\hat{\mathbf{F}}}=\mathbf{I}_{T}-\hat{\mathbf{F}}\left(\hat{\mathbf{F}}^{\prime} \hat{\mathbf{F}}\right)^{-1} \hat{\mathbf{F}}^{\prime}
$$

\footnotetext{
${ }^{6}$ This assumption can be relaxed considerably by requiring $\varepsilon_{i t}$ to follow a martingale difference process over $t$, or even to be a strong mixing process with sufficiently small mixing coefficients.
} 
and $\hat{\mathbf{F}}=\left(\hat{\boldsymbol{f}_{1}}, \hat{\boldsymbol{f}_{2}} \ldots, \hat{\boldsymbol{f}_{T}}\right)^{\prime}$. We then determine a threshold, $C_{N T}^{2}>0$, such that if, and only if, $N \hat{\sigma}_{i T}^{2}<C_{N T}^{2}$ then unit $i$ is selected as dominant. Below we proceed by analyzing the asymptotic properties of $\hat{\sigma}_{i T}^{2}$, and sketching steps that lead to a procedure that consistently, namely with probability tending to one, selects only dominant units. Formal proofs are given in the Appendix. But first we provide an overview of the literature on estimation of $\mathbf{F}$ and $\mathbf{A}$ and their asymptotic properties that we need for establishing our main theoretical results.

\subsection{Consistent estimation of $\mathrm{A}$ and $\mathrm{F}$ by principal components}

Let $\mathbf{X}=\left(\mathbf{x}_{1}, \mathbf{x}_{2}, \ldots, \mathbf{x}_{N}\right)$ be the $T \times N$ matrix of observations on $x_{i t}$ for $i=1,2, \ldots, N ; t=$ $1,2, \ldots, T$. It is well known that the $T \times T$ matrix $\mathbf{X} \mathbf{X}^{\prime}$ and the $N \times N$ matrix $\mathbf{X}^{\prime} \mathbf{X}$ have the same eigenvalues. Denote the first $p$ largest eigenvalues of these two matrices by $\left(\hat{\rho}_{1}, \hat{\rho}_{2}, \ldots, \hat{\rho}_{p}\right)$ and the associated orthonormal eigenvectors of $\mathbf{X} \mathbf{X}^{\prime}$ and $\mathbf{X}^{\prime} \mathbf{X}$ by the $T \times p$ matrix $\hat{\mathbf{P}}$ and the $N \times p$ matrix $\hat{\mathbf{Q}}$, respectively, and note that by construction $\hat{\mathbf{P}}^{\prime} \hat{\mathbf{P}}=\mathbf{I}_{p}$, and $\hat{\mathbf{Q}}^{\prime} \hat{\mathbf{Q}}=\mathbf{I}_{p}$, where $\mathbf{I}_{p}$ is an $p \times p$ identity matrix. Consider now the following $\mathrm{PC}$ estimators of $\mathbf{F}$ and $\mathbf{A}$ :

$$
\begin{aligned}
& \hat{\mathbf{A}}=\sqrt{N} \hat{\mathbf{Q}}, \\
& \hat{\mathbf{F}}=\frac{1}{\sqrt{N}} \mathbf{X} \hat{\mathbf{Q}}=\frac{1}{N} \mathbf{X} \hat{\mathbf{A}} .
\end{aligned}
$$

Given orthonormality of the eigenvectors $\hat{\mathbf{Q}}$, note that $N^{-1} \hat{\mathbf{A}}^{\prime} \hat{\mathbf{A}}=\mathbf{I}_{p}$. The factor estimator (13) satisfies

$$
\frac{\hat{\mathbf{F}}^{\prime} \hat{\mathbf{F}}}{T}=\hat{\mathbf{D}}_{N T}
$$

where $\hat{\mathbf{D}}_{N T}=(N T)^{-1} \operatorname{Diag}\left(\hat{\rho}_{1}, \hat{\rho}_{2}, \ldots, \hat{\rho}_{p}\right)$. This follows since $T^{-1} \hat{\mathbf{F}}^{\prime} \hat{\mathbf{F}}=(N T)^{-1} \hat{\mathbf{Q}}^{\prime} \mathbf{X}^{\prime} \mathbf{X} \hat{\mathbf{Q}}$, and noting that $\hat{\mathbf{Q}}$ are orthonormal eigenvectors of $\mathbf{X}^{\prime} \mathbf{X}$. Alternative estimators of $\mathbf{A}$ and $\mathbf{F}$, suggested by Bai and Ng (2002), are given by

$$
\begin{aligned}
\tilde{\mathbf{F}} & =\sqrt{T} \hat{\mathbf{P}}, \\
\tilde{\mathbf{A}} & =\frac{1}{T} \mathbf{X}^{\prime} \tilde{\mathbf{F}},
\end{aligned}
$$

where (15) satisfies $T^{-1} \tilde{\mathbf{F}}^{\prime} \tilde{\mathbf{F}}=\mathbf{I}_{p}$. Bai and $\mathrm{Ng}$ (2002) also consider the following transformation ${ }^{7}$

$$
\tilde{\mathbf{F}}=\hat{\mathbf{F}}\left(\frac{\hat{\mathbf{F}}^{\prime} \hat{\mathbf{F}}}{T}\right)^{-1 / 2}=\hat{\mathbf{F}} \hat{\mathbf{D}}_{N T}^{-1 / 2}
$$

where the last step follows from equation (14). In the first instance and to proceed with the derivation of our dominant unit detection procedure, we discuss the relationships between $\hat{\mathbf{A}}$, $\hat{\mathbf{F}}$ and $\tilde{\mathbf{A}}, \tilde{\mathbf{F}}$, and show that they are equivalent in the context of this paper. This equivalence result considerably simplifies the derivations and proofs since the probability limit results in Bai and $\mathrm{Ng}$ (2002), as well as the additional results established in the Appendix, relate to $\tilde{\mathbf{F}}$

\footnotetext{
${ }^{7}$ Note the typo in the corresponding equation in Bai and $\mathrm{Ng}$ (2002).
} 
and $\tilde{\mathbf{A}}$, whilst for the derivations of our proposed thresholding criterion, it is much simpler to work in terms of $\hat{\mathbf{F}}$ and $\hat{\mathbf{A}}$.

We first note that $\tilde{\mathbf{F}}$ and $\hat{\mathbf{F}}$ only differ by the non-singular rotation matrix $\hat{\mathbf{D}}_{N T}^{-1 / 2}$. Then, using (17) it readily follows that

$$
\mathbf{M}_{\hat{\mathbf{F}}}=\mathbf{I}_{T}-\hat{\mathbf{F}}\left(\hat{\mathbf{F}}^{\prime} \hat{\mathbf{F}}\right)^{-1} \hat{\mathbf{F}}=\mathbf{M}_{\tilde{\mathbf{F}}}=\mathbf{I}_{T}-\tilde{\mathbf{F}}\left(\tilde{\mathbf{F}}^{\prime} \tilde{\mathbf{F}}\right)^{-1} \tilde{\mathbf{F}}^{\prime}
$$

and hence

$$
\hat{\sigma}_{i T}^{2}=\frac{\mathbf{x}_{i}^{\prime} \mathbf{M}_{\hat{\mathbf{F}}} \mathbf{x}_{i}}{T}=\frac{\mathbf{x}_{i}^{\prime} \mathbf{M}_{\tilde{\mathbf{F}}} \mathbf{x}_{i}}{T}
$$

It is helpful to bear in mind the following relationship between the two sets of estimators $(\tilde{\mathbf{F}}, \tilde{\mathbf{A}})$ and $(\hat{\mathbf{F}}, \hat{\mathbf{A}})$. Note that, by Lemma A.3 in Bai $(2003), \hat{\mathbf{D}}_{N T} \stackrel{p}{\rightarrow} \mathbf{D}$, where $\mathbf{D}$ is a diagonal matrix with finite elements (see the proof of Lemma A.1 in Bai (2003)). It follows that

$$
\left\|\hat{\mathbf{D}}_{N T}\right\|_{F}=O_{p}(1) \text {. }
$$

Further, $\tilde{\mathbf{F}} \tilde{\mathbf{A}}^{\prime}=\hat{\mathbf{F}} \hat{\mathbf{A}}^{\prime}$ holds since the common component of $\mathbf{X}$, i.e. $\mathbf{F A}^{\prime}$, is uniquely determined by the separation assumption which requires $\mathbf{f}_{t}^{\prime} \mathbf{a}_{i}$ to be strongly cross-sectionally dependent whilst $v_{i t}$ is cross-sectionally weakly correlated. (See Assumptions 2 and 3). Hence, recalling the relation (17) we must have

$$
\hat{\mathbf{F}}=\tilde{\mathbf{F}} \hat{\mathbf{D}}_{N T}^{1 / 2}, \text { and } \hat{\mathbf{A}}=\tilde{\mathbf{A}} \hat{\mathbf{D}}_{N T}^{-1 / 2} \text {. }
$$

Using the estimators (15)-(16), Bai and $\mathrm{Ng}$ (2002) show in their equation (5) that

$$
\frac{1}{T}\left\|\tilde{\mathbf{F}}-\mathbf{F H}_{N T}\right\|_{F}^{2}=O_{p}\left(\frac{1}{\delta_{N T}^{2}}\right),
$$

where $\delta_{N T}^{2}=\min (N, T)$, and $\mathbf{H}_{N T}$ is a non-singular $p \times p$ matrix that could depend on $N$ and $T$, so long as its probability limit exists and is non-singular. Using (21) and the fact that $\left\|\mathbf{H}_{N T}^{-1}\right\|=O_{p}(1)$ holds by the properties of $\mathbf{H}_{N T}$, then

$$
\frac{1}{T}\left\|\mathbf{F}-\tilde{\mathbf{F}} \mathbf{Q}_{N T}\right\|_{F}^{2}=O_{p}\left(\frac{1}{\delta_{N T}^{2}}\right)
$$

where $\mathbf{Q}_{N T}=\mathbf{H}_{N T}^{-1}$, noting that this matrix is non-singular and satisfies $\left\|\mathbf{Q}_{N T}\right\|_{F}=O_{p}(1)$. Setting $\mathbf{Q}_{N T}=\hat{\mathbf{D}}_{N T}^{1 / 2}$, and noting the relation between $\tilde{\mathbf{F}}$ and $\hat{\mathbf{F}}$, we also have $T^{-1}\|\mathbf{F}-\hat{\mathbf{F}}\|_{F}^{2}=$ $O_{p}\left(\delta_{N T}^{-2}\right)$, and more generally,

$$
\frac{1}{T}\left\|\mathbf{F}-\hat{\mathbf{F}} \mathbf{S}_{N T}\right\|_{F}^{2}=O_{p}\left(\frac{1}{\delta_{N T}^{2}}\right),
$$

for any non-singular $p \times p$ matrix $\mathbf{S}_{N T}$ that satisfies $\left\|\mathbf{S}_{N T}\right\|_{F}=O_{p}(1)$. It is obvious that (21) is an important, well known, result that plays an important role in our analysis. However, we need further basic results that, to some limited extent, go beyond those existing in the literature. We provide those in the following proposition. To simplify the exposition and without loss of generality, we set $\mathbf{S}_{N T}=\mathbf{I}_{p}$. Since only the product $\mathbf{F} \mathbf{A}^{\prime}$ is identified, this restriction is innocuous and implies the normalization $N^{-1} \mathbf{A}^{\prime} \mathbf{A}=\mathbf{I}_{p}$. 
Proposition 1 Under Assumptions 1-4, and setting $\mathbf{S}_{N T}=\mathbf{I}_{p}$, we have

$$
\begin{aligned}
\left\|\mathbf{F}_{0}-\hat{\mathbf{F}}\right\|_{F} & =O_{p}\left(\frac{\sqrt{T}}{\delta_{N T}}\right), \\
\left\|\mathbf{A}_{0}-\hat{\mathbf{A}}\right\|_{F} & =O_{p}\left(\frac{\sqrt{N}}{\delta_{N T}}\right), \\
\left\|\mathbf{V}\left(\mathbf{A}_{0}-\hat{\mathbf{A}}\right)\right\|_{F} & =O_{p}\left(\frac{\sqrt{N T}}{\delta_{N T}}\right), \\
\|\mathbf{V A}\|_{F} & =O_{p}(\sqrt{N T}), \\
\left\|\mathbf{A}_{0}^{\prime}\left(\mathbf{A}_{0}-\hat{\mathbf{A}}\right)\right\| & =O_{p}\left(\frac{N}{\delta_{N T}}\right),
\end{aligned}
$$

where $\hat{\mathbf{F}}$ and $\hat{\mathbf{A}}$ are defined by (13) and (12), and $\mathbf{A}_{0}$ denotes the true value of $\mathbf{A}$, and $\delta_{N T}^{2}=$ $\min (N, T)$.

The above proposition follows from Lemmas 3-6 set out in the Appendix. For general rotation matrices $\mathbf{H}_{N T}, \mathbf{Q}_{N T}$ and $\mathbf{S}_{N T}$, Proposition 1 can be used to obtain

$$
\left\|\frac{\mathbf{V}\left(\tilde{\mathbf{A}}-\mathbf{A}_{0} \mathbf{H}_{N T}^{-1}\right)}{N}\right\|_{F}=O_{p}\left(\sqrt{\frac{T}{N}} \frac{1}{\delta_{N T}}\right) .
$$

The matrix $\mathbf{H}_{N T}$ has been introduced into the expression above in order to ensure compatibility with the results (21) and (23). Again, $\mathbf{V}\left(\tilde{\mathbf{A}}-\mathbf{A}_{0} \mathbf{H}_{N T}^{-1}\right)=\mathbf{V}\left(\tilde{\mathbf{A}} \mathbf{H}_{N T}-\mathbf{A}_{0}\right) \mathbf{H}_{N T}^{-1}$, so that

$$
\left\|\mathbf{V}\left(\tilde{\mathbf{A}} \mathbf{H}_{N T}-\mathbf{A}_{0}\right)\right\|_{F} \leq\left\|\mathbf{H}_{N T}\right\|_{F}\left\|\mathbf{V}\left(\tilde{\mathbf{A}}-\mathbf{A}_{0} \mathbf{H}_{N T}^{-1}\right)\right\|_{F}
$$

and letting $\mathbf{H}_{N T}=\hat{\mathbf{D}}_{N T}^{-1 / 2} \mathbf{S}_{N T}^{-1}$, we have

$$
\left\|\mathbf{V}\left(\mathbf{A}_{0}-\tilde{\mathbf{A}} \hat{\mathbf{D}}_{N T}^{-1 / 2} \mathbf{S}_{N T}^{-1}\right)\right\|_{F} \leq O_{p}(1) O_{p}\left[\left\|\mathbf{V}\left(\tilde{\mathbf{A}}-\mathbf{A}_{0} \mathbf{H}_{N T}^{-1}\right)\right\|_{F}\right] .
$$

Recall that $\hat{\mathbf{A}}=\tilde{\mathbf{A}} \hat{\mathbf{D}}_{N T}^{-1 / 2}$ by equation (20). Hence,

$$
\begin{aligned}
\left\|\mathbf{V}\left(\mathbf{A}_{0}-\hat{\mathbf{A}} \mathbf{S}_{N T}^{-1}\right)\right\|_{F} & =O_{p}\left[\left\|\mathbf{V}\left(\tilde{\mathbf{A}}-\mathbf{A}_{0} \hat{\mathbf{D}}_{N T}^{1 / 2} \mathbf{S}_{N T}\right)\right\|_{F}\right] \\
& =O_{p}\left(\frac{\sqrt{N T}}{\delta_{N T}}\right)
\end{aligned}
$$

by the equality $\mathbf{H}_{N T}^{-1}=\hat{\mathbf{D}}_{N T}^{1 / 2} \mathbf{S}_{N T}$ and application of result (24). This concludes our discussion of principal components estimators. The preceding results will be extensively used in the next section under the simplifying assumption $\mathbf{S}_{N T}^{-1}=\mathbf{I}_{p}$. 


\subsection{Thresholding of $\hat{\sigma}_{i T}^{2}$}

We begin by considering the asymptotic properties of $\hat{\sigma}_{i T}^{2}$, defined by (10). Using (5), we have $\mathbf{x}_{i}=\mathbf{F}_{0} \mathbf{a}_{i}$, where $\mathbf{F}_{0}$ is the $T \times m$ matrix of observations on the true factors. Hence, when unit $i$ is dominant we have

$$
\begin{aligned}
\hat{\sigma}_{i T}^{2} & =\frac{\mathbf{a}_{i}^{\prime} \mathbf{F}_{0}^{\prime} \mathbf{M}_{\hat{\mathbf{F}}} \mathbf{F}_{0} \mathbf{a}_{i}}{T} \\
& =\frac{\mathbf{a}_{i}^{\prime}\left(\mathbf{F}_{0}-\hat{\mathbf{F}}\right)^{\prime} \mathbf{M}_{\hat{\mathbf{F}}}\left(\mathbf{F}_{0}-\hat{\mathbf{F}}\right) \mathbf{a}_{i}}{T},
\end{aligned}
$$

by noting that $\mathbf{F}_{0}^{\prime} \mathbf{M}_{\hat{\mathbf{F}}} \mathbf{F}_{0}=\left(\mathbf{F}_{0}-\hat{\mathbf{F}} \mathbf{S}_{N T}\right)^{\prime} \mathbf{M}_{\hat{\mathbf{F}}}\left(\mathbf{F}_{0}-\hat{\mathbf{F}} \mathbf{S}_{N T}\right)$, for any positive definite matrix, $\mathbf{S}_{N T}$. Now, using (5) and post-multiplying both sides by $\mathbf{A}_{0}$ we also have

$$
\frac{\mathbf{X} \mathbf{A}_{0}}{N}=\frac{\mathbf{F}_{0} \mathbf{A}_{0}^{\prime} \mathbf{A}_{0}}{N}+\frac{\mathbf{V A}_{0}}{N}=\mathbf{F}_{0}+\frac{\mathbf{V A} \mathbf{A}_{0}}{N}
$$

where to derive the last step we have made use of the normalization $N^{-1} \mathbf{A}_{0}^{\prime} \mathbf{A}_{0}=\mathbf{I}_{m}$. Furthermore, since $\hat{\mathbf{F}}=N^{-1} \mathbf{X} \hat{\mathbf{A}}$ by equation (13), then

$$
\left(\mathbf{F}_{0}-\hat{\mathbf{F}}\right)=\frac{\mathbf{X}\left(\mathbf{A}_{0}-\hat{\mathbf{A}}\right)}{N}-\frac{\mathbf{V A}_{0}}{N},
$$

and

$$
\mathbf{M}_{\hat{\mathbf{F}}} \mathbf{F}_{0}=\mathbf{M}_{\hat{\mathbf{F}}}\left[\frac{\mathbf{X}\left(\mathbf{A}_{0}-\hat{\mathbf{A}}\right)}{N}-\frac{\mathbf{V A}_{0}}{N}\right]
$$

Using (5) here to substitute out $\mathbf{X}$,

$$
\begin{aligned}
\mathbf{M}_{\hat{\mathbf{F}}} \mathbf{F}_{0} & =\mathbf{M}_{\hat{\mathbf{F}}}\left[\frac{\mathbf{F}_{0} \mathbf{A}_{0}^{\prime}\left(\mathbf{A}_{0}-\hat{\mathbf{A}}\right)}{N}+\frac{\mathbf{V}\left(\mathbf{A}_{0}-\hat{\mathbf{A}}\right)}{N}\right]-\mathbf{M}_{\hat{\mathbf{F}}}\left(\frac{\mathbf{V A}}{N}\right) \\
& =\frac{\mathbf{M}_{\hat{\mathbf{F}}}\left(\mathbf{F}_{0}-\hat{\mathbf{F}}\right) \mathbf{A}_{0}^{\prime}\left(\mathbf{A}_{0}-\hat{\mathbf{A}}\right)}{N}+\frac{\mathbf{M}_{\hat{\mathbf{F}}} \mathbf{V}\left(\mathbf{A}_{0}-\hat{\mathbf{A}}\right)}{N}-\mathbf{M}_{\hat{\mathbf{F}}}\left(\frac{\mathbf{V} \mathbf{A}_{0}}{N}\right) .
\end{aligned}
$$

Using the above results in $(26), \hat{\sigma}_{i T}^{2}$ can be written as

$$
N \hat{\sigma}_{i T}^{2}=B_{i 1}+B_{i 2}+\ldots+B_{i 6}
$$


where

$$
\begin{aligned}
B_{i 1} & =\frac{\mathbf{a}_{i}^{\prime} \mathbf{A}_{0}^{\prime} \mathbf{V}^{\prime} \mathbf{M}_{\hat{\mathbf{F}}} \mathbf{V} \mathbf{A}_{0} \mathbf{a}_{i}}{N T}, \\
B_{i 2} & =2 \frac{\mathbf{a}_{i}^{\prime} \mathbf{A}_{0}^{\prime} \mathbf{V}^{\prime} \mathbf{M}_{\hat{\mathbf{F}}} \mathbf{V}\left(\mathbf{A}_{0}-\hat{\mathbf{A}}\right) \mathbf{a}_{i}}{N T}, \\
B_{i 3} & =2 \frac{\mathbf{a}_{i}^{\prime} \mathbf{A}_{0}^{\prime} \mathbf{V}^{\prime} \mathbf{M}_{\hat{\mathbf{F}}}\left(\mathbf{F}_{0}-\hat{\mathbf{F}}\right) \mathbf{A}_{0}^{\prime}\left(\mathbf{A}_{0}-\hat{\mathbf{A}}\right) \mathbf{a}_{i}}{N T}, \\
B_{i 4} & =\frac{\mathbf{a}_{i}^{\prime}\left(\mathbf{A}_{\mathbf{0}}-\hat{\mathbf{A}}\right)^{\prime} \mathbf{V}^{\prime} \mathbf{M}_{\hat{\mathbf{F}}} \mathbf{V}\left(\mathbf{A}_{0}-\hat{\mathbf{A}}\right) \mathbf{a}_{i}}{N T}, \\
B_{i 5} & =2 \frac{\mathbf{a}_{i}^{\prime}\left(\mathbf{A}_{\mathbf{0}}-\hat{\mathbf{A}}\right)^{\prime} \mathbf{V}^{\prime} \mathbf{M}_{\hat{\mathbf{F}}}\left(\mathbf{F}_{0}-\hat{\mathbf{F}}\right) \mathbf{A}_{0}^{\prime}\left(\mathbf{A}_{0}-\hat{\mathbf{A}}\right) \mathbf{a}_{i}}{N T}, \\
B_{i 6} & =\frac{\mathbf{a}_{i}^{\prime}\left(\mathbf{A}_{\mathbf{0}}-\hat{\mathbf{A}}\right)^{\prime} \mathbf{A}_{0}\left(\mathbf{F}_{0}-\hat{\mathbf{F}}\right)^{\prime} \mathbf{M}_{\hat{\mathbf{F}}}\left(\mathbf{F}_{0}-\hat{\mathbf{F}}\right) \mathbf{A}_{0}^{\prime}\left(\mathbf{A}_{0}-\hat{\mathbf{A}}\right) \mathbf{a}_{i}}{N T} .
\end{aligned}
$$

If unit $i$ is not dominant, then it is easy to see that (28) is augmented by two extra terms, given $\mathrm{by}^{8}$

$$
\begin{aligned}
B_{i 7} & =\frac{N \mathbf{v}_{i}^{\prime} \mathbf{M}_{\hat{\mathbf{F}}} \mathbf{v}_{i}}{T}, \\
B_{i 8} & =\frac{N \mathbf{a}_{i}^{\prime} \mathbf{F}_{0}^{\prime} \mathbf{M}_{\hat{\mathbf{F}}} \mathbf{v}_{i}}{T}=\frac{N \mathbf{a}_{i}^{\prime}\left(\mathbf{F}_{0}-\hat{\mathbf{F}}\right)^{\prime} \mathbf{M}_{\hat{\mathbf{F}}} \mathbf{v}_{i}}{T},
\end{aligned}
$$

which will be formally taken into account in the proof of Theorem 1 set out in Section A.2 of the Appendix. Here, we simply note that $B_{i 7}=O_{p}(N)$ and $B_{i 8}=o_{p}(N)$.

Using Lemma 1 , in the Appendix, we have, assuming that $x_{i t}$ is a dominant unit,

$$
N \hat{\sigma}_{i T}^{2}=B_{i 1}+O_{p}\left(\frac{1}{\delta_{N T}}\right)+O_{p}\left(\frac{\sqrt{N}}{\delta_{N T}^{2}}\right) .
$$

Consider now $B_{i 1}$ and note that

$$
B_{i 1}=\frac{\mathbf{a}_{i}^{\prime} \mathbf{A}_{0}^{\prime} \mathbf{V}^{\prime} \mathbf{M}_{\hat{\mathbf{F}}} \mathbf{V} \mathbf{A}_{0} \mathbf{a}_{i}}{N T} \leq \frac{\mathbf{d}_{i}^{\prime} \mathbf{V}^{\prime} \mathbf{V} \mathbf{d}_{i}}{N T}
$$

where $\mathbf{d}_{i}=\mathbf{A}_{0} \mathbf{a}_{i}$. If $x_{i t}$ is a dominant unit,

$$
\operatorname{Pr}\left(N \hat{\sigma}_{i T}^{2}>C_{N T}^{2}\right) \leq \operatorname{Pr}\left(\frac{\mathbf{d}_{i}^{\prime} \mathbf{V}^{\prime} \mathbf{V} \mathbf{d}_{i}}{N T}>C_{N T}^{2}\right)+o(1),
$$

if $\sqrt{N} / \delta_{N T}^{2} \rightarrow 0$. Note that

$$
\frac{\sqrt{N}}{\delta_{N T}^{2}}=\left\{\begin{array}{l}
\frac{\sqrt{N}}{T} \text { if } T \leq N \\
\frac{1}{\sqrt{N}} \text { otherwise }
\end{array}\right.
$$

\footnotetext{
${ }^{8}$ Note that in the absence of any dominant units $\mathbf{v}_{i}=\mathbf{u}_{i}$. In general, we use $\mathbf{v}_{i}$ (and $\mathbf{V}$ ) in line with the general factor model given by (5).
} 
and so the condition for the remainder term to vanish is $\frac{\sqrt{N}}{T} \rightarrow 0$, as $N, T \rightarrow \infty$. It is possible that this condition can be relaxed if one finds a tighter upper bound for $\left\|\mathbf{A}_{0}^{\prime}\left(\mathbf{A}_{0}-\hat{\mathbf{A}}\right)\right\|$ than result $(\mathrm{E})$ in Proposition 1 , and if stationarity is imposed on $E\left(v_{i t} v_{i t^{\prime}}\right)$, where $\mathbf{V}=\left(v_{i t}\right)$. For now, we adhere to Assumptions A-F of Bai (2003), and require that $\frac{\sqrt{N}}{T} \rightarrow 0$, as $N, T \rightarrow \infty$. Under these conditions, we focus on the first probability term in (38) and note that

$$
\mathbf{d}_{i}^{\prime} \mathbf{V}^{\prime} \mathbf{V} \mathbf{d}_{i}=\sum_{t=1}^{T}\left(\sum_{j=1}^{N} v_{j t} d_{i j}\right)^{2}=\sum_{t=1}^{T}\left(\mathbf{d}_{i}^{\prime} \mathbf{v}_{t}\right)^{2}
$$

where $\mathbf{d}_{i}=\mathbf{A}_{0} \mathbf{a}_{i}=\left(d_{i 1}, d_{i 2}, \ldots, d_{i N}\right)^{\prime}$. Note that if the panel contains $m$ dominant units, $\mathbf{v}_{t}=\left(\mathbf{0}_{1 \times m}, \mathbf{u}_{t}^{\prime}\right)^{\prime}$, where $\mathbf{u}_{t}=\mathbf{H} \boldsymbol{\varepsilon}_{t}$. See (8). Partition $\mathbf{d}_{i}=\left(\mathbf{d}_{i 1}^{\prime}, \mathbf{d}_{i 2}^{\prime}\right)^{\prime}$, where $\mathbf{d}_{i 1}$ and $\mathbf{d}_{i 2}$ are the $m \times 1$ and $n \times 1$ sub-vectors of $\mathbf{d}_{i}$ (recall that $n=N-m$ ). Hence

$$
\mathbf{d}_{i}^{\prime} \mathbf{V}^{\prime} \mathbf{V} \mathbf{d}_{i}=\sum_{t=1}^{T}\left(\mathbf{d}_{i 2}^{\prime} \mathbf{u}_{t}\right)^{2}=\sum_{t=1}^{T}\left(\mathbf{d}_{i 2}^{\prime} \mathbf{H} \varepsilon_{t}\right)^{2}
$$

where by assumption $\mathbf{H}$ is an $n \times n$ matrix with bounded row and column absolute sum norms, and $\varepsilon_{t}=\left(\varepsilon_{m+1, t}, \varepsilon_{m+2, t}, \ldots, \varepsilon_{N, t}\right) \sim \operatorname{IID}\left(\mathbf{0}, \mathbf{I}_{n}\right)$. Using the above results we can now write

$$
\begin{aligned}
\frac{\mathbf{d}_{i}^{\prime} \mathbf{V}^{\prime} \mathbf{V} \mathbf{d}_{i}}{N T} & =\left(\frac{n}{N}\right) \frac{1}{T} \sum_{t=1}^{T}\left(\frac{\mathbf{d}_{i 2}^{\prime} \mathbf{H} \varepsilon_{t}}{\sqrt{n}}\right)^{2} \\
& =\left(\frac{n}{N}\right) \frac{1}{T} \sum_{t=1}^{T}\left(\varphi_{i}^{\prime} \varepsilon_{t}\right)^{2}
\end{aligned}
$$

where $\varphi_{i}=n^{-1 / 2} \mathbf{H}^{\prime} \mathbf{d}_{i 2}=\left(\varphi_{i 1}, \varphi_{i 2}, \ldots, \varphi_{i n}\right)^{\prime}$. Let

$$
\eta_{i n}^{2}=\varphi_{i}^{\prime} \varphi_{i}=\frac{1}{n} \mathbf{d}_{i 2}^{\prime} \mathbf{H H}^{\prime} \mathbf{d}_{i 2}=\frac{1}{n} \mathbf{d}_{i 2}^{\prime} \boldsymbol{\Sigma}_{u} \mathbf{d}_{i 2},
$$

where $\boldsymbol{\Sigma}_{u}=E\left(\mathbf{u}_{t} \mathbf{u}_{t}^{\prime}\right)=\mathbf{H H}^{\prime}$ and by assumption is time-invariant. ${ }^{9}$ We also have

$$
\sup _{i} \eta_{i n}^{2} \leq \sup _{i}\left(n^{-1} \mathbf{d}_{i 2}^{\prime} \mathbf{d}_{i 2}\right) \lambda_{\max }\left(\boldsymbol{\Sigma}_{u}\right), \text { and } \inf _{i} \eta_{i n}^{2} \geq \inf _{i}\left(n^{-1} \mathbf{d}_{i 2}^{\prime} \mathbf{d}_{i 2}\right) \lambda_{\min }\left(\boldsymbol{\Sigma}_{u}\right),
$$

where by assumption $0<c<\lambda_{\min }\left(\boldsymbol{\Sigma}_{u}\right) \leq \lambda_{\max }\left(\boldsymbol{\Sigma}_{u}\right)<C<\infty$. Noting that, in view of $(6)$ and (7), we have

$$
\sup _{i}\left(n^{-1} \mathbf{a}_{i}^{\prime} \mathbf{A}_{b}^{\prime} \mathbf{A}_{b} \mathbf{a}_{i}\right) \leq \sup _{i}\left\|\mathbf{a}_{i}\right\|^{2} \lambda_{\max }\left(n^{-1} \sum_{j=m+1}^{N} \mathbf{a}_{j} \mathbf{a}_{j}^{\prime}\right)<C<\infty
$$

and

$$
\inf _{i}\left(n^{-1} \mathbf{a}_{i}^{\prime} \mathbf{A}_{b}^{\prime} \mathbf{A}_{b} \mathbf{a}_{i}\right) \geq \inf _{i}\left\|\mathbf{a}_{i}\right\|^{2} \lambda_{\min }\left(n^{-1} \sum_{j=m+1}^{N} \mathbf{a}_{j} \mathbf{a}_{j}^{\prime}\right)>c>0,
$$

we also have $\sup _{i}\left(n^{-1} \mathbf{d}_{i 2}^{\prime} \mathbf{d}_{i 2}\right)<C$, and $\inf _{i}\left(n^{-1} \mathbf{d}_{i 2}^{\prime} \mathbf{d}_{i 2}\right)>0$, and overall

\footnotetext{
${ }^{9}$ However, one can still allow for conditionally time-varying covariances.
} 


$$
\sup _{i} \eta_{i n}^{2}<C, \text { and } \inf _{i} \eta_{i n}^{2}>0, \text { for all } n .
$$

Now using (39) in (38) and applying Lemma A11 in the online supplement to Chudik, Kapetanios, and Pesaran (2018), we have

$$
\begin{aligned}
\operatorname{Pr}\left[n \hat{\sigma}_{i T}^{2}>\left(\frac{n}{N}\right) C_{N T}^{2}\right] & \leq \operatorname{Pr}\left[\sum_{t=1}^{T}\left(\varphi_{i}^{\prime} \varepsilon_{t}\right)^{2}>T\left(\frac{n}{N}\right) C_{N T}^{2}\right]+o(1) \\
& \leq \sum_{t=1}^{T} \operatorname{Pr}\left[\left(\varphi_{i}^{\prime} \varepsilon_{t}\right)^{2}>\left(\frac{n}{N}\right) C_{N T}^{2}\right]+o(1) .
\end{aligned}
$$

Additionally, letting $\varphi_{i}^{\prime} \varepsilon_{t}=\sum_{j=1}^{n} \varphi_{i j} \varepsilon_{j t}$, we can write

$$
\begin{aligned}
\operatorname{Pr}\left[\left(\varphi_{i}^{\prime} \varepsilon_{t}\right)^{2}>\left(\frac{n}{N}\right) C_{N T}^{2}\right] & =\operatorname{Pr}\left(\left|\varphi_{i}^{\prime} \varepsilon_{t}\right|>\left(\frac{n}{N}\right)^{1 / 2} C_{N T}\right) \\
& =\operatorname{Pr}\left(\left|\sum_{j=1}^{n} \varphi_{i j} \varepsilon_{j t}\right|>\left(\frac{n}{N}\right)^{1 / 2} C_{N T}\right) .
\end{aligned}
$$

In order to proceed from the above expression, we note that under Assumption $3, \operatorname{Var}\left(\sum_{j=1}^{n} \varphi_{i j} \varepsilon_{j t}\right)=$ $\sum_{j=1}^{n} \varphi_{i j}^{2}=\eta_{i n}^{2}$, and

$$
\operatorname{Pr}\left(\left|\varepsilon_{j t}\right|>a\right) \leq C_{0} \exp \left(-C_{1} a^{s}\right)
$$

for all $a>0, s>0$ and some fixed constants $C_{0}$ and $C_{1}$. This allows us to apply Lemma A3 of Chudik, Kapetanios, and Pesaran (2018) to obtain

$$
\operatorname{Pr}\left(\left|\sum_{j=1}^{n} \varphi_{i j} \varepsilon_{j t}\right|>C_{N T}\right) \leq \exp \left[\frac{-(1-\pi)^{2} C_{N T}^{2}}{2 \eta_{i n}^{2}}\right],
$$

for some $0<\pi<1$ and $C_{N T}=O\left(n^{\lambda}\right)$, with $0<\lambda<\frac{s+1}{s+2}$ (note that $n / N=1-m / N \approx 1$ ), where using (44) in (43) and assuming that unit $i$ is dominant yields

$$
\operatorname{Pr}\left(N \hat{\sigma}_{i T}^{2}>\left(\frac{n}{N}\right) C_{N T}^{2}\right) \leq T \exp \left[\frac{-(1-\pi)^{2} C_{N T}^{2}}{2 \eta_{i n}^{2}}\left(\frac{n}{N}\right)\right]+o(1),
$$

for some $0<\pi<1$ and $\eta_{i n}^{2}$ as defined by (40). Hence,

$$
\operatorname{Pr}\left[N \hat{\sigma}_{i T}^{2} \leq\left(\frac{n}{N}\right) C_{N T}^{2} \mid i \text { is dominant }\right] \geq 1-\exp \left[\log (T)-\frac{(1-\pi)^{2} C_{N T}^{2}}{2 \eta_{i n}^{2}}\left(\frac{n}{N}\right)\right],
$$

and

$$
\operatorname{Pr}\left[N \hat{\sigma}_{i T}^{2}>\left(\frac{n}{N}\right) C_{N T}^{2} \mid i \text { is dominant }\right] \rightarrow 0,
$$

as $N, T \rightarrow \infty$, and $\frac{\sqrt{N}}{T} \rightarrow 0$, and if (note that $n / N \rightarrow 1$ )

$$
\log (T)-\frac{(1-\pi)^{2} C_{N T}^{2}}{2 \eta_{i n}^{2}} \rightarrow-\infty .
$$


This last condition is satisfied if (again setting $n / N$ to unity)

$$
C_{N T}^{2}>\frac{2 \log (T) \eta_{i n}^{2}}{(1-\pi)^{2}}
$$

or if

$$
C_{N T}^{2}=2 C \eta_{i n}^{2} \log (T),
$$

for some $C>1$. Accordingly, $i$ can be selected as a dominant unit if, for some $C>1$, $\hat{\sigma}_{i T}^{2} \leq \frac{2 C \eta_{i n}^{2} \log (T)}{N}$.

We note further that in the Appendix we formally show that all remainder terms in (37), will not exceed the threshold, with probability approaching one if the unit is dominant. We also show that $B_{i 7}=O_{p}(N)$ and $B_{i 8}=o_{p}(N)$, and further, using (52), that the residual variance, $\hat{\sigma}_{i T}^{2}$, will exceed the threshold with probability approaching one if the unit is not dominant.

An important issue relates to the estimation of $\eta_{i n}^{2}$. Since $n=N-m$ and in practice $m$ is not known, at the estimation stage we assume $m=0$, and note that under $m=0$, then $\eta_{i N}^{2}=\frac{1}{N} \mathbf{a}_{i}^{\prime} \mathbf{A}_{0}^{\prime} \boldsymbol{\Sigma}_{u} \mathbf{A}_{0} \mathbf{a}_{i}$ for which a consistent estimator can be obtained using the PC estimators of $\mathbf{a}_{i}$ and $\mathbf{A}_{0}$, and a suitable threshold estimator of $\boldsymbol{\Sigma}_{v}$. Recall that when $\boldsymbol{\Sigma}_{u}=\mathbf{H H}^{\prime}$ and since by assumption $\mathbf{H}$ is a row and column bounded (see Assumption 3), then $\boldsymbol{\Sigma}_{u}$ is also rowbounded and hence satisfies usual sparsity conditions assumed in the literature on estimation of large covariance matrices (see, e.g., El Karoui, 2008 or Bickel and Levina, 2008). Then, $\eta_{i N}^{2}$ can be consistently estimated by

$$
\hat{\eta}_{i N}^{2}=N^{-1} \hat{\mathbf{a}}_{i}^{\prime} \hat{\mathbf{A}}^{\prime} \tilde{\mathbf{\Sigma}}_{u} \hat{\mathbf{A}} \hat{\mathbf{a}}_{i}
$$

where $\hat{\mathbf{A}}$ is given by (12), $\hat{\mathbf{a}}_{i}$ is the OLS estimator of $\mathbf{a}_{i}$ in the regression of $\mathbf{x}_{i}$ (the selected dominant unit) on $\hat{\mathbf{F}}$, where the latter is given by $(13)$, and $\tilde{\boldsymbol{\Sigma}}_{u}=\left(\tilde{\sigma}_{i j}\right)$ is a consistent estimator of $\boldsymbol{\Sigma}_{u}$. Here we use the multiple testing estimator of Bailey, Pesaran, and Smith (2018) given by

$$
\begin{aligned}
& \tilde{\sigma}_{i j}=\hat{\sigma}_{i j} I\left(\hat{\rho}_{i j}>\frac{c_{\pi}(N)}{\sqrt{T}}\right), c_{\pi}(N)=\Phi^{-1}\left(1-\frac{\pi}{2 N^{\delta}}\right), \\
& \hat{\sigma}_{i j}=\frac{1}{T} \sum_{t=1}^{T} \hat{u}_{i t} \hat{u}_{j t}, \hat{\rho}_{i j}=\frac{\hat{\sigma}_{i j}}{\hat{\sigma}_{i i}^{1 / 2} \hat{\sigma}_{j j}^{1 / 2}},
\end{aligned}
$$

where $\hat{u}_{i t}, t=1,2, \ldots, T$ are the OLS residuals from the regression of $x_{i t}$ (the selected dominant unit) on $\hat{\mathbf{F}}$ (including an intercept in all regressions), $\Phi^{-1}(\cdot)$ is the inverse of cumulative distribution function of a standard normal variate, $\pi$ is the nominal size for the multiple testing procedure, which we set to $1 \%$, and $\delta$ is set to 1.5 , which allows for possible departures from Gaussian errors, $u_{i t}$. Other estimators can also be used such as the universal thresholding by El Karoui (2008) and Bickel and Levina (2008), and the adaptive thresholding by Cai and Liu (2011).

Our threshold detection algorithm, referred to as $\sigma^{2}$ thresholding, can be summarized as follows:

Algorithm 1 Let $\mathbf{x}_{i}$ be the $T \times 1$ vector of observations on the $i$-th unit in the panel, and $\mathbf{X}=\left(\mathbf{x}_{1}, \mathbf{x}_{2}, \ldots, \mathbf{x}_{N}\right)$ be the $T \times N$ matrix of observations on all the $N$ units in the panel. Suppose that $p \leq p_{\max }$, where $p_{\max }$ is selected a priori to be sufficiently large. Compute $\hat{\mathbf{F}}=$ 
$\frac{1}{\sqrt{N}} \mathbf{X} \hat{\mathbf{Q}}$, where $\hat{\mathbf{Q}}$ is the $N \times p_{\max }$ matrix whose columns are the orthonormal eigenvectors of $\mathbf{X}^{\prime} \mathbf{X}$, such that $N^{-1} \hat{\mathbf{Q}}^{\prime} \hat{\mathbf{Q}}=\mathbf{I}_{p_{\max }}$. Compute $\hat{\mathbf{a}}_{i}, \hat{v}_{i t}$ and $\hat{\sigma}_{i T}^{2}$ to be the OLS estimator, residual and residual variance of the regression of $\mathbf{x}_{i}$ on $\hat{\mathbf{F}}$, namely

$$
\begin{aligned}
\hat{\mathbf{a}}_{i} & =\left(\hat{\mathbf{F}}^{\prime} \hat{\mathbf{F}}\right)^{-1} \hat{\mathbf{F}}^{\prime} \mathbf{x}_{i}, \\
\hat{\mathbf{u}}_{i} & =\left(\hat{u}_{i 1}, \hat{u}_{i 2}, \ldots, \hat{u}_{i T}\right)^{\prime}=\mathbf{M}_{\hat{\mathbf{F}}} \mathbf{x}_{i}=\left[\mathbf{I}_{T}-\hat{\mathbf{F}}\left(\hat{\mathbf{F}}^{\prime} \hat{\mathbf{F}}\right)^{-1} \hat{\mathbf{F}}^{\prime}\right] \mathbf{x}_{i}, \\
\hat{\sigma}_{i T}^{2} & =T^{-1} \mathbf{x}_{i}^{\prime} \mathbf{M}_{\hat{\mathbf{F}}} \mathbf{x}_{i} .
\end{aligned}
$$

Then, select unit $i$ to be dominant if

$$
\hat{\sigma}_{i T}^{2} \leq \frac{2 \hat{\eta}_{i N}^{2} \log T}{N}
$$

where $\hat{\eta}_{i N}^{2}$ is given by (45).

The following theorem provides a formal summary statement of the preceding analysis.

Theorem 1 Suppose that observations on $x_{i t}$, for $i=1,2, \ldots, N$, and $t=1,2, \ldots, T$ are generated according to the general linear factor model given by (1) and (2) with $m$ dominant units. Let $I_{D}$ be the set of indices of the dominant units, and $I_{N D}$ its complement, with $I_{D}$ allowed to be an empty set. Denote by $\hat{I}_{D}$ and $\hat{I}_{D N}$ their estimates based on the threshold criteria (46). Let Assumptions 1-4 hold and $\frac{\sqrt{N}}{T} \rightarrow 0$. Then as $N$ and $T \rightarrow \infty$, jointly, we have

$$
\lim _{N, T \rightarrow \infty} \operatorname{Pr}\left(\left\{\hat{I}_{D}=I_{D}\right\} \cap\left\{\hat{I}_{N D}=I_{N D}\right\}\right)=1
$$

This theorem establishes that the proposed error variance threshold criterion is consistent, in the sense that it correctly selects the dominant (if any) and the non-dominant units asymptotically.

Remark 3 Note that both the theoretical exposition above and the formal arguments of the Appendix apply both to the case of no external factors as well as the case where all units are affected by a finite number of external factors, represented by $\mathbf{g}_{t}$ in (1) and (2).

\section{A sequential, multiple testing version of the $\sigma^{2}$ thresh- olding}

The $\sigma^{2}$ thresholding procedure, has good but not exceptional small sample properties as we illustrate in the online supplement to this article. However, it provides a basis for further development. The first point to note is that while the method is good at detecting the presence of dominant units, in general it tends to pick too many units as dominant. Finite sample adjustments are needed to achieve a more conservative detection outcome. A simple and effective refinement of the main method is a sequential algorithm that detects dominant units one at a time. Considering a sequential algorithm suggests the use of dominant units that have 
been identified at earlier steps of the procedure as observed factors. This reduces the number of unobserved factors to be estimated given a maximum number of considered factors, $p_{\max }$. Therefore, the static factor model (5) employed to conduct $\sigma^{2}$ thresholding is replaced by the augmented factor model

$$
x_{i t}=\mathbf{f}_{t}^{* \prime} \mathbf{a}_{i}^{*}+\mathbf{x}_{a t}^{* \prime} \mathbf{b}_{a i}^{*}+v_{i t}, t=1,2, \ldots, T ; i=1, \ldots N_{1},
$$

where $\mathbf{x}_{a t}^{*}$ is a $r \times 1$ vector of identified dominant units (the row $t$ of the $T \times r$ matrix $\mathbf{X}_{a}^{*}$ ), $\mathbf{f}_{t}^{*}$ is a $p_{\max }-r$ vector of unobserved common factors and $v_{i t}$ constitutes the idiosyncratic variation of unit $i$ at time point $t$. With regards to the procedure of Section 4.2, the role of $\sigma^{2}$ thresholding is not to determine the number and the identities of the dominant units directly. Instead, $\sigma^{2}$ thresholding is used to determined whether or not there is evidence of remaining dominant units in the data, given the dominant units that have been identified. Being initiated with $r=0$ (i.e. no identified dominant units), $N_{1}=N-r=N$ and some chosen value of $p_{\max }$ subject to the condition $p_{\max } \geq m+1$, the sequential algorithm, referred to as $S-\sigma^{2}$ thresholding is an iteration of the following two steps:

Algorithm 2 1. Conduct $\sigma^{2}$ thresholding using model (47), with $m^{*}=p_{\max }-r$ estimated factors. Let $\widetilde{m}$ be the estimated number of dominant units estimated using Algorithm 1. If $\widetilde{m}=0$, stop and conclude that there are $r$ dominant units.

2. If $\hat{m}>0$, obtain $i^{*}=\arg \min _{i} \hat{\sigma}_{i}^{2}$. Append $\mathbf{x}_{i^{*}}$ to $\mathbf{X}_{a}^{*}$ and drop $\mathbf{x}_{i^{*}}$ from $\mathbf{X}$. Update $r$ to $r+1$ and $N_{1}$ to $N_{1}-1$.

The two steps are repeated until either $\widetilde{m}=0$ in the first step or $r=p_{\max }$ at the end of step 2. The number of dominant units is then $\hat{m}=r$ and their identities correspond to the indices of the columns in the initial $T \times N$ vector $\mathbf{X}$ that are found in the $T \times r$ matrix $\mathbf{X}_{a}^{*}=\left(\mathbf{x}_{a 1}^{*} ; \ldots ; \mathbf{x}_{a T}^{*}\right)^{\prime}$.

Effectively the method constructs residuals of the remaining units on the selected units and repeats the selection on these residuals. The use of residuals in the algorithm's steps requires further theoretical refinements. These are discussed in Section A.3 of the Appendix where it is shown that our proposed threshold is valid only if $N<T$, which is a more restrictive condition than that of Theorem 1. In particular, we prove the following result, in Section A.3 of the Appendix.

Corollary 1 Suppose that observations on $x_{i t}$, for $i=1,2, \ldots, N$, and $t=1,2, \ldots, T$ are generated according to the general linear factor model given by (1) and (2) with $m$ dominant units. Let $I_{D}$ be the set of indices of the dominant units, and $I_{N D}$ its complement, with $I_{D}$ allowed to be an empty set. Denote by $\hat{I}_{D}$ and $\hat{I}_{D N}$ their estimates based on $S-\sigma^{2}$ thresholding. Let Assumptions 1-4 hold and $\frac{N}{T} \rightarrow 0$. Then as $N$ and $T \rightarrow \infty$, jointly, we have

$$
\lim _{N, T \rightarrow \infty} \operatorname{Pr}\left(\left\{\hat{I}_{D}=I_{D}\right\} \cap\left\{\hat{I}_{N D}=I_{N D}\right\}\right)=1 .
$$

If $N \geq T$, then an alternative threshold could be considered. This is given by

$$
\hat{\sigma}_{i T}^{2} \leq \frac{2 \hat{\sigma}_{i u}^{2} \log T}{T}
$$


where $\hat{\sigma}_{i u}^{2}=T^{-1} \sum_{t=1}^{T}\left(x_{i t}-\mathbf{X}_{a t}^{* \prime} \hat{\gamma}_{i}^{*}\right)^{2}$, with $\hat{\gamma}_{i}^{*}$ being the estimated vector of slope coefficients from a regression of $\mathbf{M}_{\hat{\mathbf{F}}} \mathbf{x}_{i}$ on $\mathbf{M}_{\hat{\mathbf{F}}} \mathbf{X}_{a}^{*}$. This is justified in Section A.3. As its small sample properties are inferior to those of our main procedure we do not pursue this further in the main paper but only in the online supplement. However, it is important to note that it provides a theoretical justification for our general methodology when $N>T$.

Finally, the sequential algorithm can be supplemented with an additional multiple testing hurdle in order to reduce the risk of falsely detecting a dominant unit in small samples. Analogous to the plain sequential algorithm discussed above, the extended algorithm is initiated with $r=0, N_{1}=N$ and some chosen value of $p_{\max }$ subject to the condition $p_{\max } \geq m+1$. It consists of the following five steps which are repeated until the estimated number of dominant units $\widetilde{m}$ in the first step is equal to zero:

Algorithm 3 1. Conduct $\sigma^{2}$ thresholding using model (47) and $m^{*}=p_{\max }-r$ estimated factors. Let $\widetilde{m}$ be the estimated number of dominant units estimated using Algorithm 1. If $\widetilde{m}=0$, stop and conclude that there are $r$ dominant units.

2. If $\widetilde{m}>0$, obtain $i^{*}=\arg \min _{i} \hat{\sigma}_{i}^{2}$. For each $j=1, \ldots i^{*}-1, i^{*}+1, \ldots, N_{1}$ estimate the model

$$
x_{j t}=x_{i^{*} t} \gamma_{j}^{*}+\mathbf{f}_{t}^{* \prime} \mathbf{a}_{j}^{*}+\mathbf{x}_{a t}^{* \prime} \mathbf{b}_{a j}^{*}+v_{j t}, t=1,2, \ldots, T,
$$

where the unobserved factors $\mathbf{f}_{t}^{*}$ are estimated by the eigenvectors associated to the $p_{\max }-$ $r-1$ largest eigenvalues of $\mathbf{X}_{-i^{*}} \mathbf{X}_{-i^{*}}^{\prime}$ with $\mathbf{X}_{-i^{*}}=\left(\mathbf{x}_{1} ; \ldots ; \mathbf{x}_{i^{*}-1} ; \mathbf{x}_{i^{*}+1} ; \ldots ; \mathbf{x}_{N_{1}}\right)$.

3. Carry out $N_{1}-1$ individual $t$ tests to check the statistical significance of the slope parameters $\hat{\gamma}_{j}^{*}$ for all $j \neq i$ in (48), using the multiple testing critical value, $\Phi^{-1}\left[1-\frac{\pi}{2\left(N_{1}-2\right)}\right]$, where the nominal size of the individual tests, $\pi$, is chosen by the investigator. In our analysis we set $\pi=0.01$.

4. Let $M$ denote the number of rejections among these $N_{1}-1$ tests. If $\log (M) / \log (N) \leq 1 / 2$, stop and conclude that there are $r$ dominant units.

5. If $\log (M) / \log (N)>1 / 2$, append $\mathbf{x}_{i^{*}}$ to $\mathbf{X}_{a}^{*}$ and eliminate $\mathbf{x}_{i^{*}}$ from $\mathbf{X}$. Update $r$ to $r+1$ and $N_{1}$ to $N_{1}-1$

We refer to this algorithm as Sequential-MT $\sigma^{2}$ thresholding or $S M T-\sigma^{2}$ thresholding for short. Note that the rule $\log (M) / \log (N) \leq 1 / 2$, or $M \leq N^{1 / 2}$ is motivated by that fact that if a factor enters only $M$ units, where $M=o\left(N^{1 / 2}\right)$, then, it is considered to be a very weak factor, and under certain conditions, it is not detectable using principal components - see, e.g., Bailey, Kapetanios, and Pesaran (2016). Again, after stopping the sequential algorithm, the number of dominant units is $\hat{m}=r$ and their identities correspond to the indices of the columns in the initial $T \times N$ vector $\mathbf{X}$ that are found in the $T \times r$ matrix $\mathbf{X}_{a}^{*}=\left(\mathbf{x}_{a 1}^{*} ; \ldots ; \mathbf{x}_{a T}^{*}\right)^{\prime}$. The additional multiple testing step ends up being very effective in small samples and is therefore our preferred approach. While we do not provide a fully rigorous proof for the consistency properties of the multiple testing step we refer the reader to Chudik, Kapetanios, and Pesaran (2018) where a full analysis of multiple testing, within a multiple regression setting, is provided. From that analysis and, in particular, Theorem 1 of that paper, it readily follows that the multiple testing step selects with probability approaching one, as $N, T \rightarrow \infty$, only dominant units. 


\section{A Comparative analysis of detection procedures by Monte Carlo simulations}

Using Monte Carlo simulations we now investigate the small sample performance of our new method relative to the methods proposed by Parker and Sul (2016, henceforth PS) and Brownlees and Mesters (2018, BM in the following). ${ }^{10}$ The PS method yields identical outcomes irrespective of whether the observations are standardized to have in sample zero means and unit variances or not. Our proposed method, being based on residuals, is not affected by demeaning of the observations and the scaling is done through the determination of the unitspecific thresholds, and hence standardization will not be an issue. In contrast, BM's detection method can be quite sensitive to standardization in finite samples, although asymptotically it should not matter whether the individual series in the panel are standardized. The BM method is also applied either including all the $N$ units, or only the $N / 2$ most connected units when selecting the dominant units. ${ }^{11}$ Accordingly, we consider four variants of the BM method: modified and unmodified with and without standardization. We shall refer to these variants as $B M$ and $B M$ (standardized) when only the $N / 2$ most connected units are considered at the selection stage, and unmodified BM and unmodified $B M$ (standardized) when all the $N$ units are included when selecting the dominant units. In the paper we focus on the modified version of BM, and give the results for their unmodified version in the online supplement. It is clear that fewer units will be detected when the modified version is used, even though the effect of standardization is less clear cut. Amongst the various $\sigma^{2}$ thresholding procedures discussed, we focus on SMT $-\sigma^{2}$ thresholding as described by Algorithm $3 .^{12}$

In accordance with the formal presentation in Section 3, we simulate the dominant unit model as

$$
\begin{aligned}
\mathbf{x}_{t a} & =\boldsymbol{\mu}_{a}+\boldsymbol{\Lambda}_{a} \mathbf{g}_{t}+\mathbf{h}_{t} \\
\mathbf{x}_{t b} & =\boldsymbol{\mu}_{b}+\mathbf{B} \mathbf{x}_{t a}+\boldsymbol{\Lambda}_{b} \mathbf{g}_{t}+\mathbf{u}_{t}
\end{aligned}
$$

for $t=1,2, \ldots, T$. The $N \times 1$ vector of fixed effects, $\boldsymbol{\mu}=\left(\boldsymbol{\mu}_{a} ; \boldsymbol{\mu}_{b}\right)^{\prime}$, are drawn from $\operatorname{IIDU}(0,1)$. The $k_{0} \times 1$ vectors $\mathbf{g}_{t}$, for $t=1,2, \ldots, T$, representing the unobserved common factors, are generated as $\mathbf{g}_{t}=\mathbf{R}_{g}^{1 / 2}\left(\mathbf{g}_{*, t}-2 \boldsymbol{\tau}_{k}\right) / 2$, where $\boldsymbol{\tau}_{k}=(1,1, \ldots, 1)^{\prime}, \mathbf{g}_{*, t}$ is a $k \times 1$ vector generated as $I I D \chi^{2}(2), \mathbf{R}_{g}^{1 / 2}$ is the square root of the $k_{0} \times k_{0}$ matrix $\mathbf{R}_{g}$ defined by

$$
\mathbf{R}_{g}=\left(1-\rho_{g}\right) \mathbf{I}_{k}+\rho_{g} \boldsymbol{\tau}_{k} \boldsymbol{\tau}_{k}^{\prime},
$$

where $\rho_{g}$ represents the pair-wise correlation coefficients of the distinct $(i, j)$ elements of $\mathbf{g}_{t}$, assumed to be the same across all $i$ and $j=1,2, . ., k$. Specifically, $\operatorname{Cov}\left(\mathbf{g}_{t}\right)=\mathbf{R}_{g}$. Similarly, the $m_{0} \times 1$ vector $\mathbf{h}_{t}$ is generated analogously as

$$
\mathbf{h}_{t}=\mathbf{R}_{h}^{1 / 2}\left(\mathbf{h}_{*, t}-2 \tau_{m}\right) / 2, \mathbf{R}_{h}=\left(1-\rho_{h}\right) \mathbf{I}_{m}+\rho_{h} \boldsymbol{\tau}_{m} \boldsymbol{\tau}_{m}^{\prime}
$$

\footnotetext{
${ }^{10}$ The detection methods of Parker and Sul and Brownless and Mesters are described in some detail in the online supplement.

${ }^{11}$ In their simulation analysis BM seem to be using the unmodified version of their method without standardization, whilst in their empirical applications they apply the modified version after standardization. See Section 6 of Brownlees and Mesters (2018).

${ }^{12}$ Simulation results for other two variants of $\sigma^{2}$ thresholding, described by Algorithms 1 and 2 , are provided in the online supplement.
} 
where $\mathbf{h}_{*, t} \sim I I D \chi^{2}(2)$. It follows that $\operatorname{Cov}\left(\mathbf{h}_{t}\right)=\mathbf{R}_{h}$, and $\rho_{h}$ represents the pair-wise correlations of the elements of $\mathbf{h}_{t}$, assumed to be the same across all pairs. The $m_{0} \times k_{0}$ matrix $\boldsymbol{\Lambda}_{a}$ and the $n \times k_{0}$ matrix $\boldsymbol{\Lambda}_{b}$ are obtained as $I I D U(0,1)$. The correlation coefficients $\rho_{g}$ and $\rho_{h}$ are drawn from $U(0.2,0.8)$, and are allowed to vary across replications.

The importance of the dominant units for the non-dominant units is represented by the $\left(N-m_{0}\right) \times m_{0}$ loading matrix $\mathbf{B}=\left(b_{i j}\right)$. To allow for the possibility of both strong and weak dominant units, $b_{i j}$ are generated as

$$
b_{i j}\left\{\begin{array}{ll}
\sim \operatorname{IIDU}(0,1) & \text { if } i \leq\left\lfloor\left(N-m_{0}\right)^{\alpha}\right\rfloor \\
=0 & \text { otherwise. }
\end{array} \text { for } i=1,2, \ldots, N-m_{0} ; j=1,2, \ldots, m_{0},\right.
$$

where as introduced in (4), $\alpha$ is the exponent that measures the degree of dominance of $\mathbf{x}_{t a}$ in the panel. For the sake of simplicity, all dominant units are assumed to have the same degree of dominance so that a subscript on $\alpha$ is redundant. When $\alpha=1$ the units are dominant, in the sense that they have non-zero effects on all the $N-m_{0}$ non-dominant units. This is the standard case in the common factor literature and ensures that $\lim _{N \rightarrow \infty}\left(N-m_{0}\right)^{-1} \mathbf{B}^{\prime} \mathbf{B}$ is a positive definite $m_{0} \times m_{0}$ matrix. This condition clearly breaks down when $\alpha<1$. As we noted before, $\mathbf{x}_{t a}$ are then referred to as influential units. ${ }^{13}$

The errors $\mathbf{u}_{t}=\left(u_{i t}\right)$ are generated as heterogeneous first order autoregressive processes

$$
u_{i t}=\rho_{i} u_{i t-1}+\left(1-\rho_{i}^{2}\right)^{1 / 2} \varepsilon_{i t}, \text { for } t=-49, \ldots, 0,1,2, \ldots, T ; i=1,2, \ldots, N-m_{0},
$$

where $\rho_{i} \sim \operatorname{IIDU}(0.2,0.5)$. The errors $\varepsilon_{i t}$ are allowed to be cross-sectionally weakly correlated. To achieve this we set $\varepsilon_{t}=\left(\varepsilon_{1 t}, \varepsilon_{2 t}, \ldots, \varepsilon_{n t}\right)^{\prime}=\boldsymbol{\Sigma}^{1 / 2} \mathbf{R}_{u}^{1 / 2} \boldsymbol{\zeta}_{t}, n=N-m$, with $\Sigma=\operatorname{diag}\left(\sigma_{11}, \sigma_{22}, \ldots, \sigma_{n n}\right)$, and

$$
\mathbf{R}_{u}=\left(\begin{array}{ccccc}
1 & \rho_{u} & \rho_{u}^{2} & \ldots & \rho_{u}^{n-1} \\
\rho_{u} & 1 & \rho_{u} & \ldots & \rho_{u}^{n-2} \\
\rho_{u}^{2} & \rho_{u} & 1 & \ldots & \rho_{u}^{n-3} \\
\vdots & \vdots & \vdots & \ddots & \vdots \\
\rho_{u}^{n-1} & \rho_{u}^{n-2} & \rho_{u}^{n-3} & \ldots & 1
\end{array}\right) .
$$

We set $\rho_{u}=0.5, \sigma_{i i}=\sigma_{*, i i} / 4+0.5$, and $\sigma_{*, i i} \sim I I D \chi^{2}(2)$, thus ensuring that $E\left(\sigma_{i i}\right)=1$. Lastly, $\zeta_{i t}=\left(\zeta_{*, i t}-2\right) / 2$, where $\zeta_{*, i t} \sim I I D \chi^{2}(2)$. In order to avoid dependence of $\mathbf{u}_{t}$ on its starting values we discard the first 50 observations. All random variables are redrawn at the start of each replication of the simulation experiments.

We carry out all the different experiments for the following $N$ and $T$ combinations:

$$
N \in\{50,100,200,500\} \text { and } T \in\{60,110,210,250\} .
$$

These $N$ and $T$ values allow for both cases where $T>N$, which is required for the BM procedure to be applicable, as well as when $T<N$, which often arises in empirical applications, and can be considered using our proposed method and the PS procedure.

\footnotetext{
${ }^{13} \mathrm{~A}$ unit is viewed as weakly dominant if it affects a number of cross section units, but the number of the units that it affects does not rise as fast as the total number of units in the panel (network). See also Chudik, Pesaran, and Tosetti (2011).
} 
The above setup allows us to control the number of dominant units, $m_{0}$, the number of external factors, $k_{0}$, as well as the degree of dominance of the dominant unit, $\alpha$. We consider all $m_{0} \leq 2$ and $k_{0} \leq 2$ combinations, namely

$$
\left\{m_{0}, k_{0}\right\}=\{0,0\},\{0,1\},\{0,2\},\{1,0\},\{1,1\},\{1,2\},\{2,0\},\{2,1\},\{2,2\} .
$$

In cases where $m_{0}>0$, we experiment with two values of $\alpha=1$ and $\alpha=0.8$. Our theoretical derivations relate to the case of strongly dominant units, namely when $\alpha=1$. However, in practice it is more likely that the dominant units are not strong, but still quite influential, which we represent by the choice of $\alpha=0.8$. In the production network literature where the degree of the dominance can be computed from input-ouput tables, $\alpha$ is estimated to lie in the region of 0.7 - 0.8. See Acemoglu, Carvalho, Ozdaglar, and Tahbaz-Salehi (2012) and Pesaran and Yang (2016, Definition 1).

Finally, all simulations in this section are conducted with 2,000 replications.

\subsection{MC results}

The first scenario to consider is one without any dominant units $\left(m_{0}=0\right)$. The results for SMT $-\sigma^{2}$ and the PS procedures are summarized in Table 1, which gives the empirical frequency of correctly estimating $m_{0}$ to be 0 . This table does not include the detection procedure proposed by BM, since the BM method pre-assumes that $m_{0}>0$, and therefore always incorrectly selects at least one dominant unit. As can be seen from this table, the SMT $-\sigma^{2}$ thresholding performs very well, even in the presence of external common factor (namely when $k_{0}>0$ ), so long as $N$ is sufficiently large. It is only outperformed by the PS procedure when $N$ is small $(N=50)$ and there are external factors $\left(k_{0}>0\right)$. Table 2 reports the average number of non-dominant units (across replications) that are falsely selected as dominant by SMT $-\sigma^{2}$, PS and BM. In this regard, SMT $-\sigma^{2}$ and PS perform perfectly when there are no external factors $\left(k_{0}=0\right)$, and register a small number of incidence of false discovery when $k_{0}=1$, and $N$ relatively small. However, the PS procedure seems to break down when the number of external factors is increased to $k_{0}=2$, and its average number of false discoveries reaches 41 with $N=500$ and $T=250$. However, the SMT $-\sigma^{2}$ thresholding continues to perform well even for $k_{0}=2$. As can be seen from Table 2, the average number of false discoveries of SMT $-\sigma^{2}$ thresholding is at most 0.7 over all values of $N$ and $T$, and tends to zero as $N$ is increased. By contrast, the BM procedure will always falsely selects non-dominant units as dominant even for panels with $N$ and $T$ large (subject to $T>N$ ). The average number of false discoveries for the BM procedure lies in range of 3 to 4 , and is unaffected by standardization. However, modification of the BM procedure seems to play a crucial role in controlling the number of false discoveries. If we use the unmodified version of BM the average number of false discoveries rise dramatically and can reach around 100 for $N=200$ and $T=250$, with standardization only helping marginally. See Section S5 of the online supplement for details.

Consider now cases where the DGP contains one or two dominant units. Table 3 reports the empirical frequency of correctly estimating the number and the identity of the dominant units by all the three detection procedures. The top panel of the table gives the results for all the three detection procedures when there is one dominant unit $\left(m_{0}=1\right)$, with and without external 
Table 1: Empirical frequency of correctly identifying the absence of a dominant unit

\begin{tabular}{|c|c|c|c|c|c|c|c|c|c|}
\hline \multicolumn{6}{|c|}{$\begin{array}{c}\mathrm{SMT}-\sigma^{2} \\
k_{0}=0\end{array}$} & \multicolumn{4}{|c|}{$\begin{array}{c}\text { PS } \\
k_{0}=0\end{array}$} \\
\hline $\mathrm{N} \backslash \mathrm{T}$ & 60 & 110 & 210 & 250 & $\mathrm{~N} \backslash \mathrm{T}$ & 60 & 110 & 210 & 250 \\
\hline 50 & 100 & 100 & 100 & 100 & 50 & 99.4 & 99.2 & 99.6 & 99.8 \\
\hline 100 & 100 & 100 & 100 & 100 & 100 & 100 & 100 & 100 & 100 \\
\hline 200 & 100 & 100 & 100 & 100 & 200 & 100 & 100 & 100 & 100 \\
\hline 500 & 100 & 100 & 100 & 100 & 500 & 100 & 100 & 100 & 100 \\
\hline \multicolumn{6}{|c|}{$k_{0}=1$} & \multicolumn{4}{|c|}{$k_{0}=1$} \\
\hline $\mathrm{N} \backslash \mathrm{T}$ & 60 & 110 & 210 & 250 & $\mathrm{~N} \backslash \mathrm{T}$ & 60 & 110 & 210 & 250 \\
\hline 50 & 88.4 & 86.4 & 82.7 & 80.3 & 50 & 53.2 & 92.0 & 97.3 & 97.7 \\
\hline 100 & 94.1 & 92.3 & 90.7 & 88.9 & 100 & 75.5 & 98.5 & 100 & 100 \\
\hline 200 & 99.8 & 99.2 & 99.4 & 99.2 & 200 & 90.6 & 100 & 100 & 100 \\
\hline 500 & 100 & 100 & 100 & 100 & 500 & 92.9 & 100 & 100 & 100 \\
\hline \multicolumn{6}{|c|}{$k_{0}=2$} & \multicolumn{4}{|c|}{$k_{0}=2$} \\
\hline $\mathrm{N} \backslash \mathrm{T}$ & 60 & 110 & 210 & 250 & $\mathrm{~N} \backslash \mathrm{T}$ & 60 & 110 & 210 & 250 \\
\hline 50 & 61.6 & 55.9 & 47.7 & 44.3 & 50 & 81.0 & 80.1 & 69.5 & 69.5 \\
\hline 100 & 84.0 & 74.5 & 64.2 & 60.9 & 100 & 86.6 & 85.7 & 63.1 & 57.4 \\
\hline 200 & 98.6 & 97.7 & 94.2 & 94.1 & 200 & 82.5 & 66.1 & 46.3 & 39.7 \\
\hline 500 & 100 & 100 & 100 & 99.9 & 500 & 99.4 & 46.8 & 22.6 & 17.6 \\
\hline
\end{tabular}

Notes: SMT $-\sigma^{2}$ thresholding is implemented using Algorithm 3 , with $p_{\max }=$
$m_{0}+k_{0}+1$, where $m_{0}$ is the true number of dominant units (if any) and $k_{0}$ is
the number of external factors. Threshold in the $\sigma^{2}$ thresholding step is given by
$\hat{\sigma}_{i T}^{2} \leq 2 \hat{\eta}_{i N}^{2} N^{-1} \log (T)$. PS refers to the Parker and Sul $(2016)$ method by setting
the number of potential dominant units to $N / 10$ per estimated factor, with the num-
ber of factors selecting using $I C_{p 2}$ criterion of Bai and $\mathrm{Ng}(2002)$. See also online
supplement.

factors, namely for $k_{0}=0,1$ and 2 . The lower part of the table gives the empirical frequencies when $m_{0}=2$, and $k_{0}=0,1$ and 2 . For the BM procedure we are only able to provide results when $T>N$. The relative performance of the three detection procedures very much depends on whether the observations are affected by an external factor, and the relative sizes of $N$ and $T$. For example, the PS method works very well only if $m_{0}=1$ and $k_{0}=0$, and breaks down completely if there are external factors or if there is more than one dominant unit. The BM method performs well when it is known that $m_{0} \geq 1$ and $T>N$. By contrast, our proposed method works reasonably well for all values of $m_{0}$ and $k_{0}$, and continues to be applicable even if $T<N$. Amongst the three methods considered only the SMT $-\sigma^{2}$ thresholding method is able to select the true dominant units with probability approaching unity as both $N$ and $T$ become large. Not surprisingly, the small sample performance of SMT $-\sigma^{2}$ thresholding deteriorates as the number of common factors, be it dominant units or external factors, is increased. In Table 4 we again consider the average number of false discoveries. The results are similar to the ones obtained earlier, with SMT $-\sigma^{2}$ procedure performing best overall. It is also interesting to note that standardization of observations affect the BM procedure adversely. This is particularly pronounced when $m_{0}=2$. Again, the modification of the BM procedure is critical for its performance. When the BM procedure is applied without modification we again obtain a large number of false discoveries, as can be seen from the results in Section S5 of the online supplement. 
Table 2: Average number of non-dominant units falsely selected as dominant $\left(m_{0}=0\right)$

\begin{tabular}{|c|c|c|c|c|c|c|c|c|c|c|c|c|c|c|}
\hline \multirow[b]{2}{*}{$\mathrm{N} \backslash \mathrm{T}$} & \multicolumn{4}{|c|}{$\begin{array}{c}k_{0}=0 \\
\mathrm{SMT}-\sigma^{2}\end{array}$} & \multicolumn{6}{|c|}{$\begin{array}{c}k_{0}=1 \\
\mathrm{SMT}-\sigma^{2}\end{array}$} & \multicolumn{4}{|c|}{$\begin{array}{c}k_{0}=2 \\
\mathrm{SMT}-\sigma^{2}\end{array}$} \\
\hline & 60 & 110 & 210 & 250 & $\mathrm{~N} \backslash \mathrm{T}$ & 60 & 110 & 210 & 250 & $\mathrm{~N} \backslash \mathrm{T}$ & 60 & 110 & 210 & 250 \\
\hline 50 & 0 & 0 & 0 & 0 & 50 & 0.1 & 0.2 & 0.2 & 0.2 & 50 & 0.4 & 0.5 & 0.6 & 0.7 \\
\hline 100 & 0 & 0 & 0 & 0 & 100 & 0.1 & 0.1 & 0.1 & 0.1 & 100 & 0.2 & 0.3 & 0.4 & 0.4 \\
\hline 200 & 0 & 0 & 0 & 0 & 200 & 0 & 0 & 0 & 0 & 200 & 0 & 0 & 0.1 & 0.1 \\
\hline 500 & 0 & 0 & 0 & 0 & 500 & 0 & 0 & 0 & 0 & 500 & 0 & 0 & 0 & 0 \\
\hline & \multicolumn{4}{|c|}{ PS } & \multicolumn{6}{|c|}{ PS } & \multicolumn{4}{|c|}{ PS } \\
\hline $\mathrm{N} \backslash \mathrm{T}$ & 60 & 110 & 210 & 250 & $\mathrm{~N} \backslash \mathrm{T}$ & 60 & 110 & 210 & 250 & $\mathrm{~N} \backslash \mathrm{T}$ & 60 & 110 & 210 & 250 \\
\hline 50 & 0 & 0 & 0 & 0 & 50 & 0.9 & 0.2 & 0.2 & 0.1 & 50 & 0.7 & 1.2 & 1.8 & 1.8 \\
\hline 100 & 0 & 0 & 0 & 0 & 100 & 0.3 & 0 & 0 & 0 & 100 & 1.0 & 1.4 & 3.7 & 4.2 \\
\hline 200 & 0 & 0 & 0 & 0 & 200 & 0.1 & 0 & 0 & 0 & 200 & 3.2 & 6.7 & 10.7 & 12.0 \\
\hline \multirow[t]{2}{*}{500} & 0 & 0 & 0 & 0 & 500 & 0.1 & 0 & 0 & 0 & 500 & 0 & 26.2 & 38.4 & 41.0 \\
\hline & \multicolumn{4}{|c|}{$\mathrm{BM}$} & \multicolumn{6}{|c|}{$\mathrm{BM}$} & \multicolumn{4}{|c|}{ BM } \\
\hline $\mathrm{N} \backslash \mathrm{T}$ & 60 & 110 & 210 & 250 & $\mathrm{~N} \backslash \mathrm{T}$ & 60 & 110 & 210 & 250 & $\mathrm{~N} \backslash \mathrm{T}$ & 60 & 110 & 210 & 250 \\
\hline 50 & 4.1 & 3.7 & 4.7 & 4.9 & 50 & 3.9 & 4.0 & 4.5 & 4.9 & 50 & 3.9 & 3.8 & 4.5 & 4.7 \\
\hline 100 & $\mathrm{n} / \mathrm{a}$ & 3.6 & 3.6 & 4.1 & 100 & $\mathrm{n} / \mathrm{a}$ & 3.5 & 3.7 & 4.2 & 100 & $\mathrm{n} / \mathrm{a}$ & 3.7 & 3.6 & 4.0 \\
\hline 200 & $\mathrm{n} / \mathrm{a}$ & $\mathrm{n} / \mathrm{a}$ & 3.2 & 3.1 & 200 & $\mathrm{n} / \mathrm{a}$ & $\mathrm{n} / \mathrm{a}$ & 3.2 & 3.0 & 200 & $\mathrm{n} / \mathrm{a}$ & $\mathrm{n} / \mathrm{a}$ & 3.1 & 3.0 \\
\hline \multirow[t]{2}{*}{500} & \multirow{2}{*}{\multicolumn{4}{|c|}{ BM (standardized) }} & 500 & $\mathrm{n} / \mathrm{a}$ & $\mathrm{n} / \mathrm{a}$ & $\mathrm{n} / \mathrm{a}$ & $\mathrm{n} / \mathrm{a}$ & 500 & $\mathrm{n} / \mathrm{a}$ & $\mathrm{n} / \mathrm{a}$ & $\mathrm{n} / \mathrm{a}$ & $\mathrm{n} / \mathrm{a}$ \\
\hline & & & & & \multicolumn{6}{|c|}{ BM (standardized) } & \multicolumn{4}{|c|}{ BM (standardized) } \\
\hline $\mathrm{N} \backslash \mathrm{T}$ & 60 & 110 & 210 & 250 & $\mathrm{~N} \backslash \mathrm{T}$ & 60 & 110 & 210 & 250 & $\mathrm{~N} \backslash \mathrm{T}$ & 60 & 110 & 210 & 250 \\
\hline 50 & 4.1 & 3.4 & 3.3 & 3.2 & 50 & 3.9 & 3.3 & 3.6 & 3.9 & 50 & 3.6 & 3.4 & 3.5 & 3.6 \\
\hline 100 & $\mathrm{n} / \mathrm{a}$ & 4.1 & 3.0 & 2.9 & 100 & $\mathrm{n} / \mathrm{a}$ & 3.4 & 3.4 & 3.4 & 100 & $\mathrm{n} / \mathrm{a}$ & 3.2 & 3.3 & 3.4 \\
\hline 200 & $\mathrm{n} / \mathrm{a}$ & $\mathrm{n} / \mathrm{a}$ & 3.4 & 2.8 & 200 & $\mathrm{n} / \mathrm{a}$ & $\mathrm{n} / \mathrm{a}$ & 3.0 & 2.8 & 200 & $\mathrm{n} / \mathrm{a}$ & $\mathrm{n} / \mathrm{a}$ & 3.0 & 2.7 \\
\hline 500 & $\mathrm{n} / \mathrm{a}$ & $\mathrm{n} / \mathrm{a}$ & $\mathrm{n} / \mathrm{a}$ & $\mathrm{n} / \mathrm{a}$ & 500 & $\mathrm{n} / \mathrm{a}$ & $\mathrm{n} / \mathrm{a}$ & $\mathrm{n} / \mathrm{a}$ & $\mathrm{n} / \mathrm{a}$ & 500 & $\mathrm{n} / \mathrm{a}$ & $\mathrm{n} / \mathrm{a}$ & $\mathrm{n} / \mathrm{a}$ & $\mathrm{n} / \mathrm{a}$ \\
\hline
\end{tabular}

Notes: The SMT $-\sigma^{2}$ and PS methods are as described in the notes to Table 1. BM refers to the modified detection method used in Section 6 of Brownlees and Mesters (2018). BM (standardized) stands for application of BM to data that have been recentered and rescaled so that each cross-section specific time series has an average of zero and a variance of one. BM methods are not applicable (n/a) if $T<N$.

The above findings continue to hold when the DGP contains influential units instead of dominant units. Table 5 reports the results for models with influential rather than strong dominant units, where the exponent of cross-sectional dependence of the dominant unit(s), is set to $\alpha=0.8$ instead of $\alpha=1$. (see (51) for a definition of $\alpha$ ). Not surprisingly, the empirical frequency of correctly identifying the true influential units is generally lower as compared to the case where the dominant units are strong. Nevertheless, SMT $-\sigma^{2}$ thresholding and BM procedure perform reasonably well even in this case. Of course, the BM method is applicable only in the case of panels with $T>N$ and if it is known that $m_{0}>0$. In cases where both BM and SMT $-\sigma^{2}$ thresholding are applicable, the proposed method seems to perform somewhat better, particularly when $T-N$ is not that large. Finally, considering the average number of non-dominant units, falsely selected, in Table 6 we again note very similar patterns to those present in Table 4, with SMT- $\sigma^{2}$ again performing best.

\section{Empirical Applications}

In this section we present empirical applications that showcase our proposed detection methodology. We consider three different applications, and report the dominant units (if any) selected 


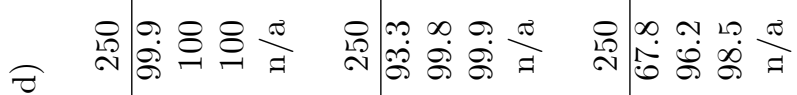

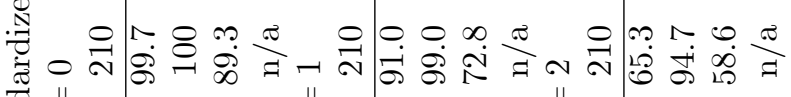
चै।

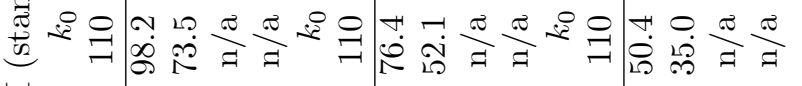
$\sum_{\oplus}$

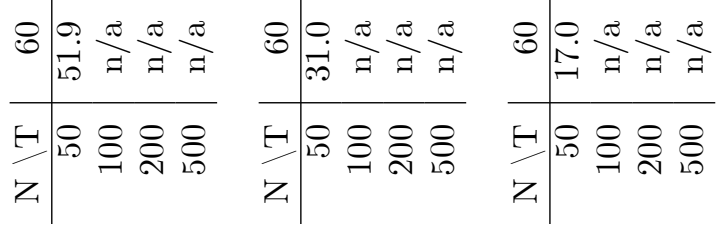

온

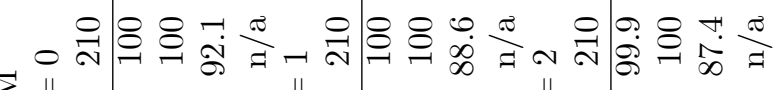
$\sum^{m}$

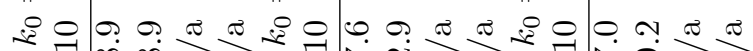

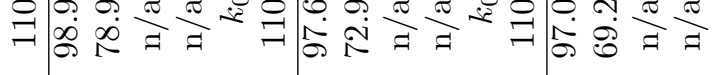

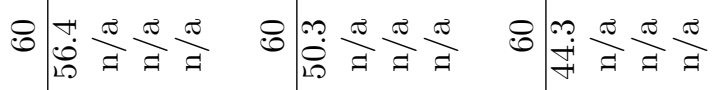

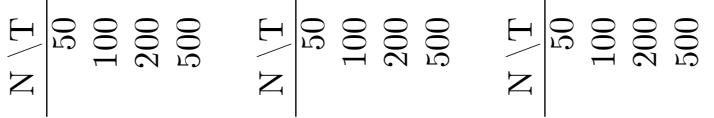

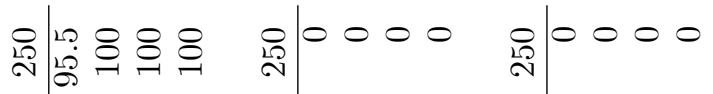

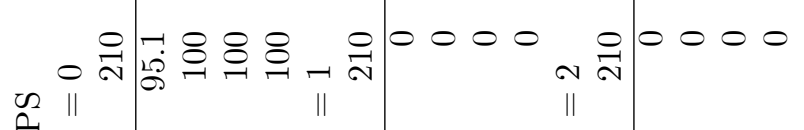

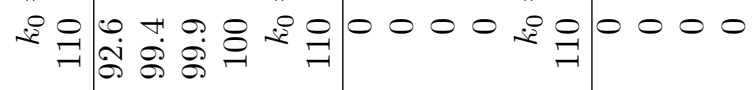

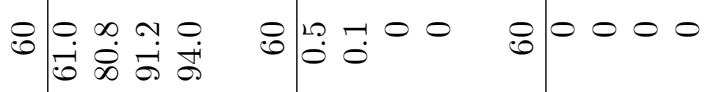

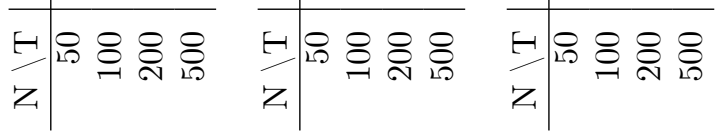

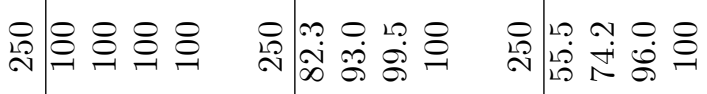
ن 卢

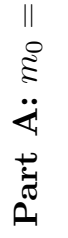

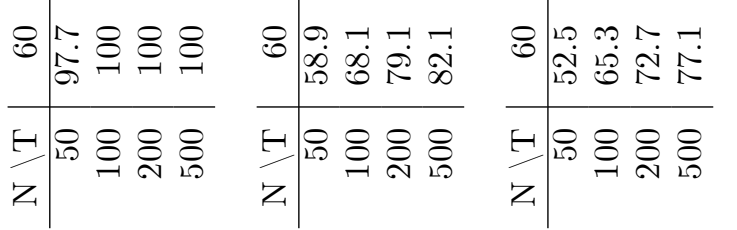

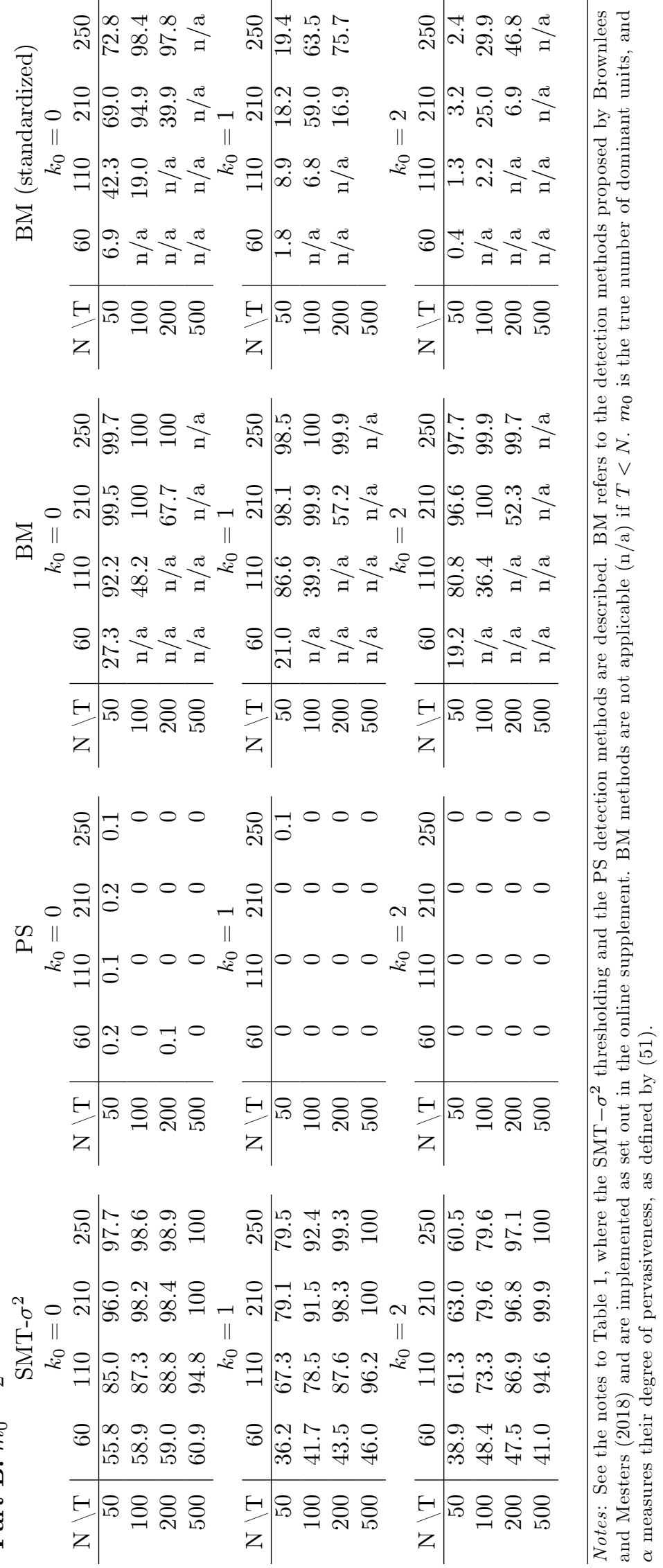


马 预 曾

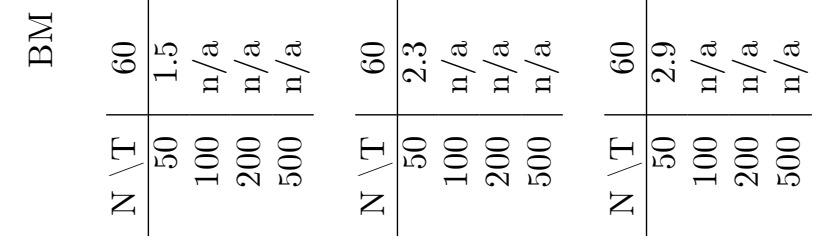

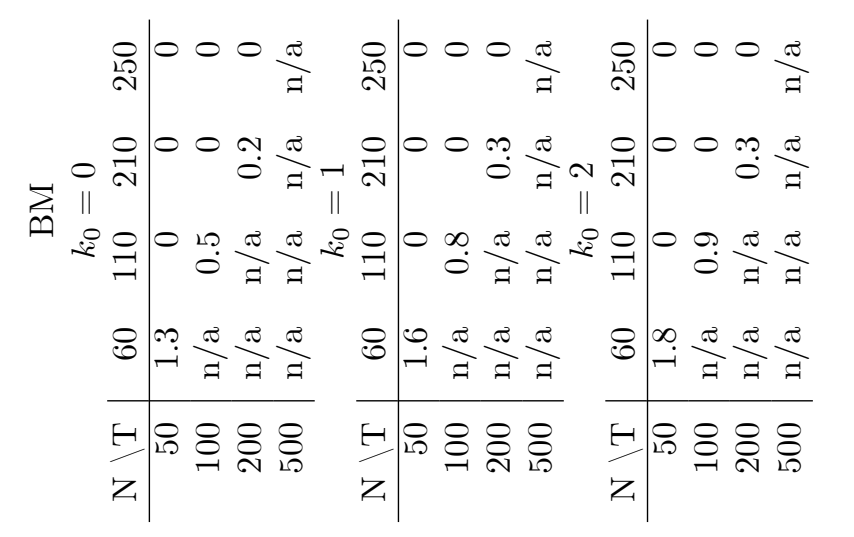

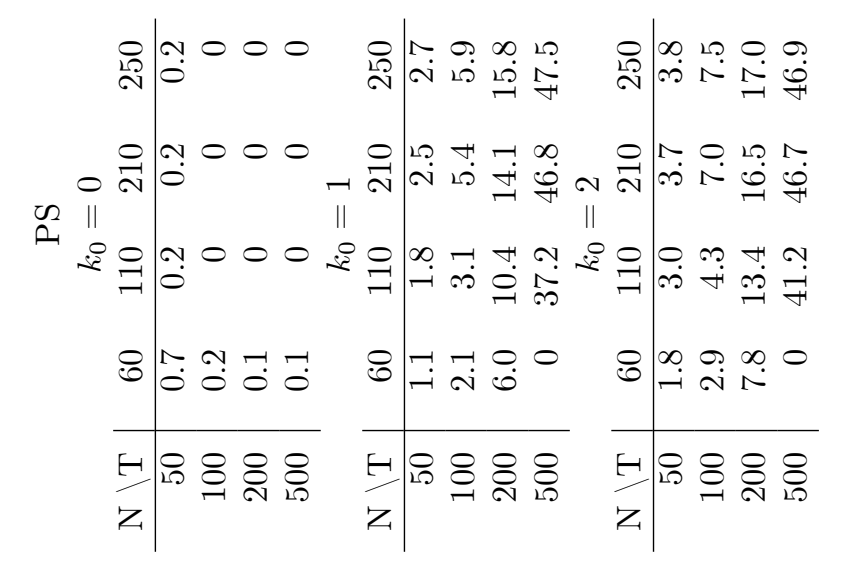

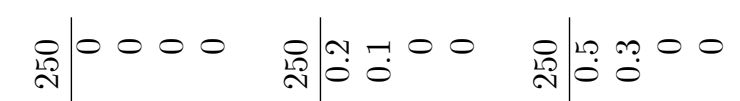

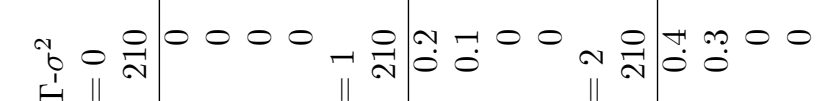
-

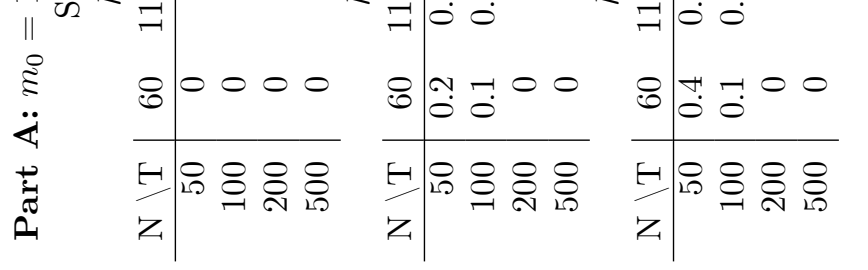
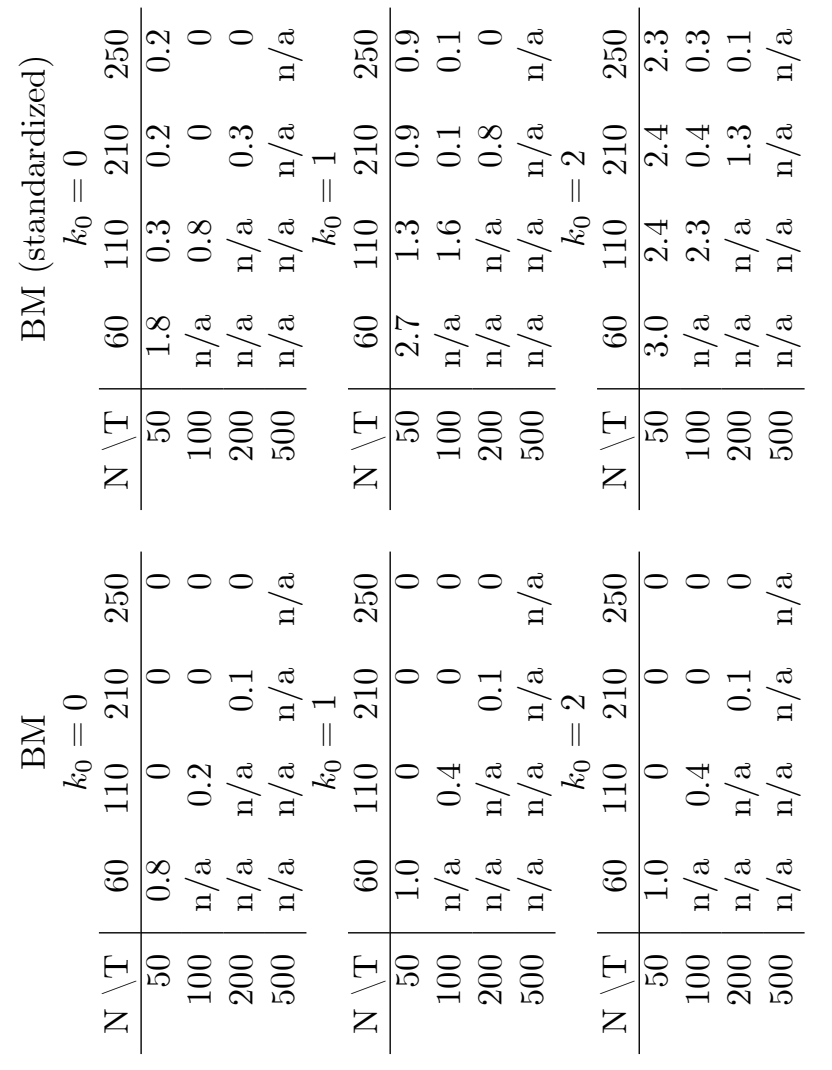

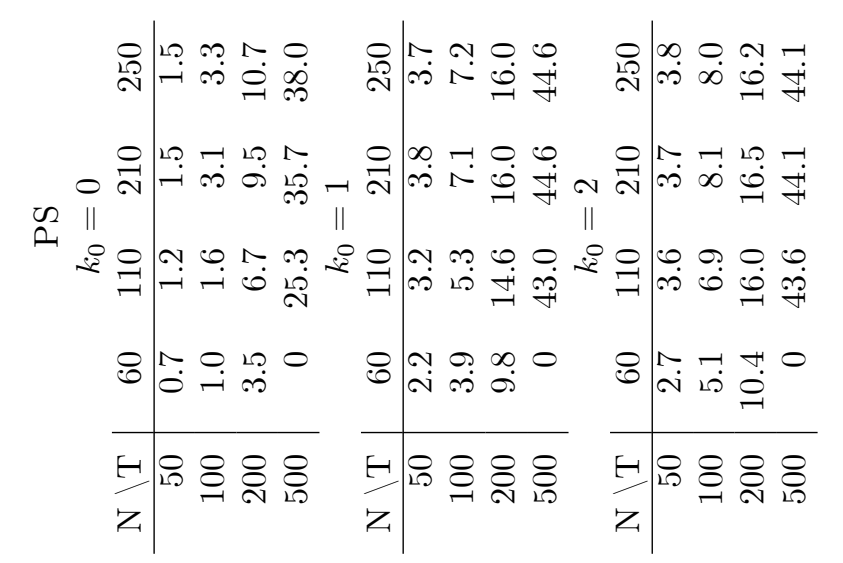

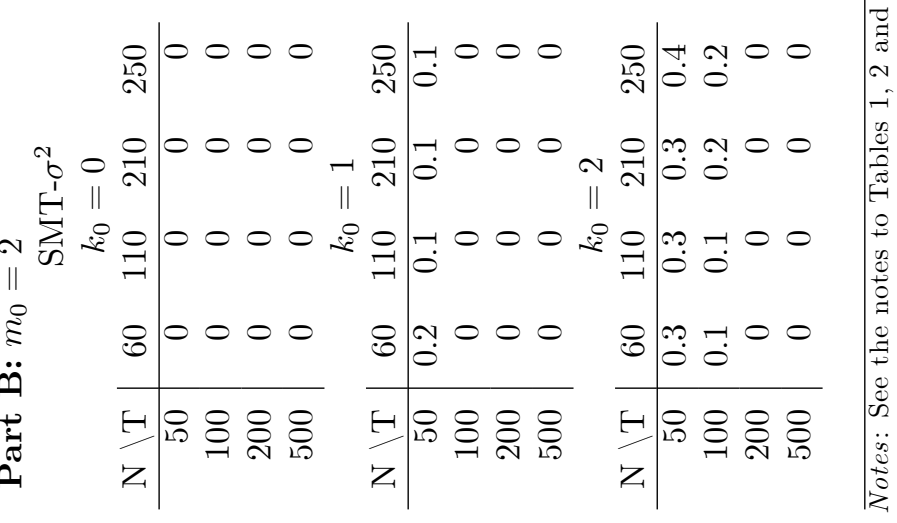




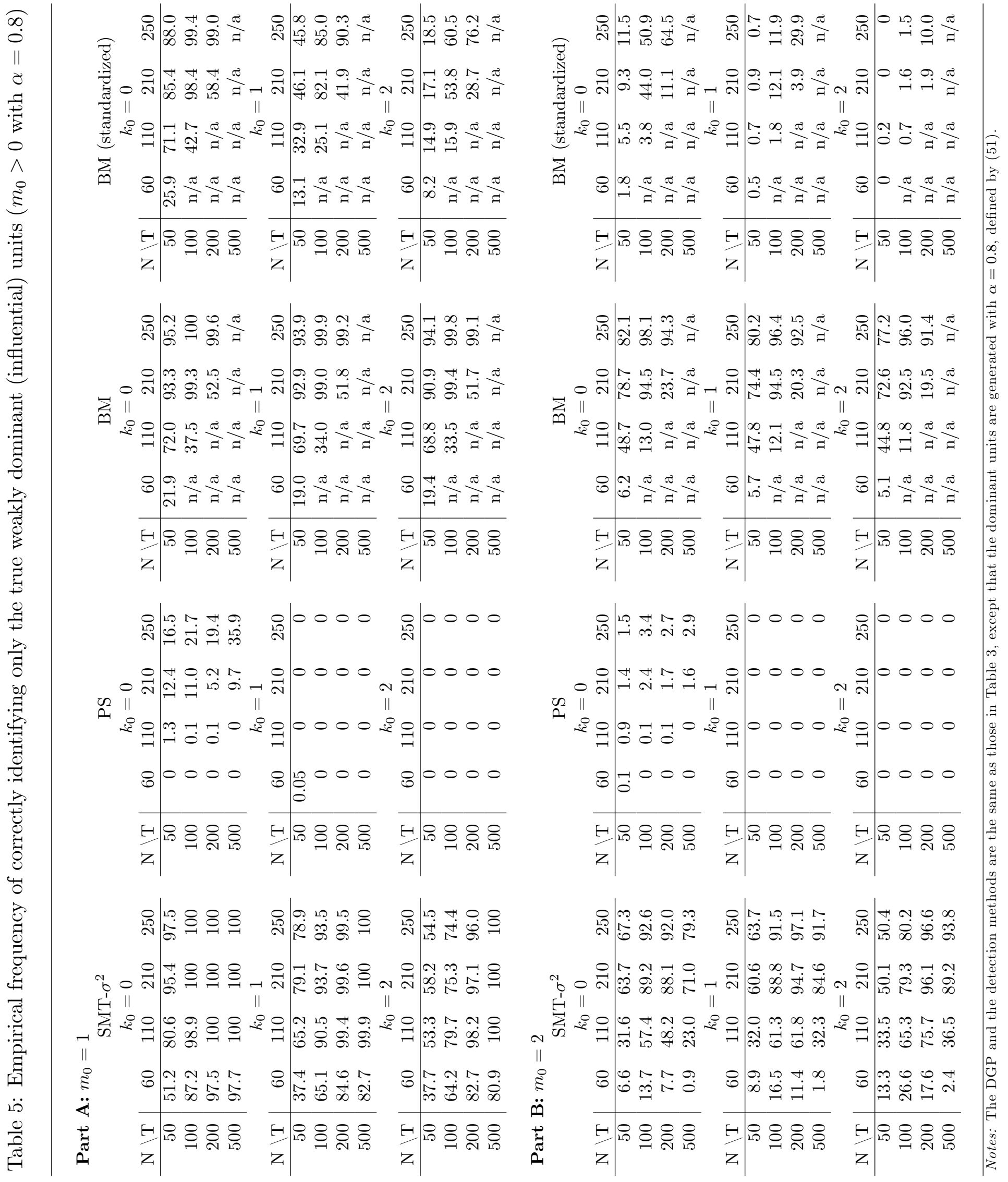




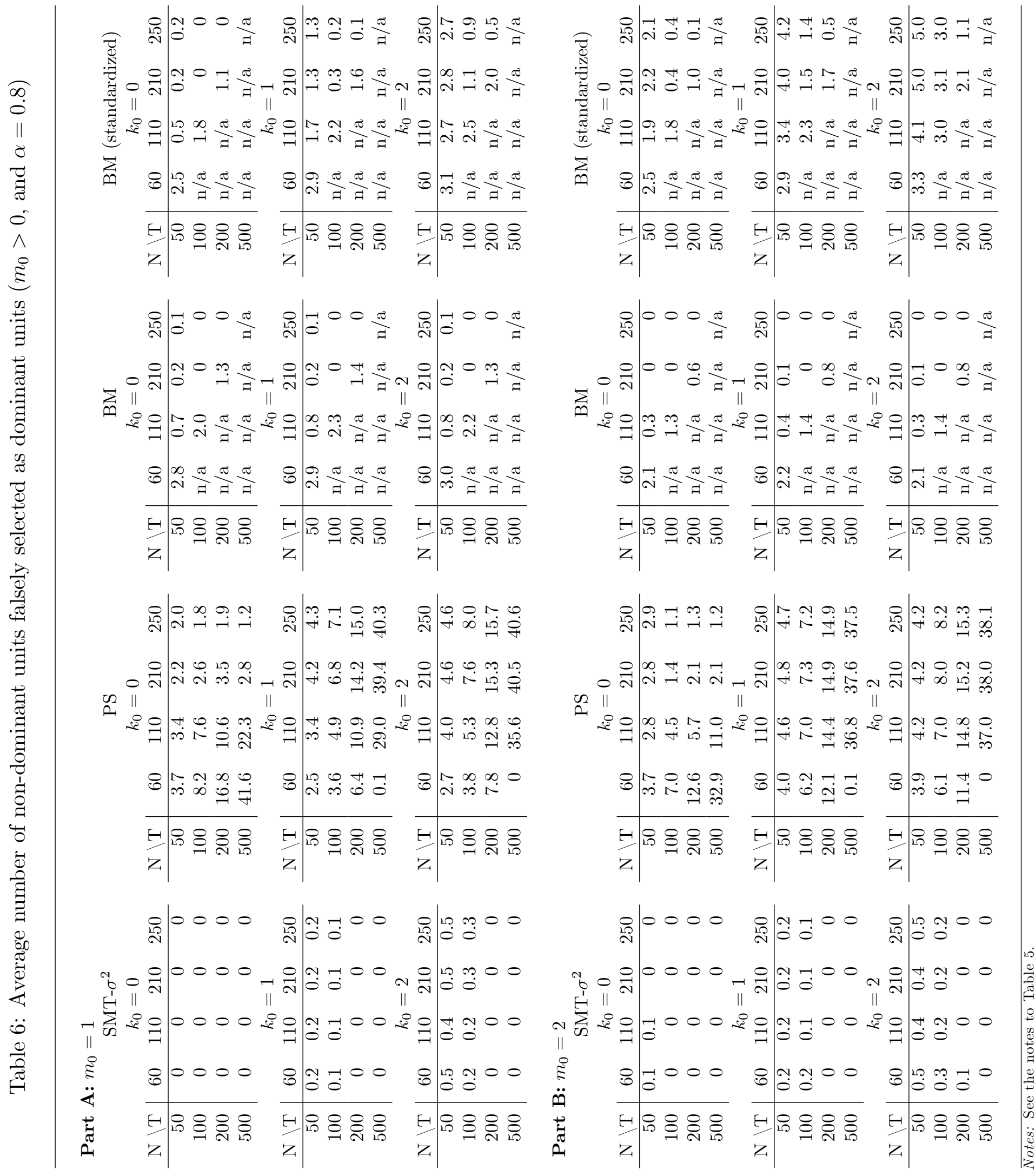


by SMT $-\sigma^{2}$ thresholding, as well as the methods of Parker and Sul (2016) and Brownlees and Mesters (2018). As in the MC section, we focus on the modified version of the BM procedure (where selection is based only on the $N / 2$ most connected units), but report results with and without standardization of the individual time series. ${ }^{14}$

\subsection{U.S. industrial production}

We begin with a panel of monthly observations on production of $N=138$ industrial sectors of the U.S. economy over the period 1972m1-2007m12. This data set has been compiled by Foerster, Sarte, and Watson (2011), and used by Brownlees and Mesters (2018) to study the presence of dominant production sectors in the U.S. ${ }^{15}$ As noted previously, by construction BM method will end up finding at least one dominant sector. In fact, Brownlees and Mesters (2018) find between 2 and 5 dominant sectors, predominantly related to the production of light motor vehicles and aluminum products. They arrive at these results by applying their modified detection procedure to sectoral growth rates after standardization. In addition to determining which sectors are dominant, the authors rank different sectors according to their level of dominance by ordering the column norms of the inverse sample covariance matrix. A comparison of this ranking with one based on the explanatory power of estimated common factors on sector-specific series is provided, revealing substantial differences in the suggested list of highly influential sectors.

We apply all the three detection methods to the full dataset as well as to the two subsamples, 1972m1-1983m12 and 1984m1-2007m12, investigated in Foerster, Sarte, and Watson (2011). For application of the PS method we selected the number of factors using the $I C_{p 2}$ criterion of Bai and $\mathrm{Ng}$ (2002). We set the maximum number of factors to 10 and obtain 1 common factor for the full sample and the first sub-sample, and 2 common factors for the second sub-sample. In application of the SMT $-\sigma^{2}$ we do not need to estimate the number of factors, but set a maximum value for $p=m+k$. To this end and to cover a wide range of possible factors, and to check the robustness of the SMT $-\sigma^{2}$ thresholding to the choice of $p_{\max }$, we tried all the values of $p_{\max }$ in the range $\{2,3,4,5,6\}$.

The results are summarized in Table 7 . The top panel of the table gives the results for the full sample, followed by the two sub-sample results. Starting with SMT $-\sigma^{2}$ thresholding, we find that no sector is identified as dominant, with the result being robust to the choice of $p_{\max }$ and the sample period. This conclusion is in line with the estimates obtained by Pesaran and Yang (2016) who make use of input-output tables for the whole U.S. economy. The PS procedure arrives at the same outcome and does not detect any dominant sector when the full sample is used, but identifies Plastic Products as dominant in the first sub-sample, and as many as 19 sectors as dominant in the second sub-sample. The list of these 19 sectors is given at the bottom of Table 7, and includes a diverse array of sectors such as Cheese, Breweries, Plastic Products, Shipping Containers, and more.

The results from the application of the BM procedure are mixed and depend on whether the observations are standardized, and the sample period considered. ${ }^{16}$ As can be seen from the

\footnotetext{
${ }^{14}$ Estimation results for unmodified BM without restrictions on the maximum number of dominant units can be found in Section S6 of the online supplement.

${ }^{15}$ In their study, Foerster, Sarte, and Watson (2011) make use of a quarterly version of this data set, and BM choose monthly frequency to ensure $T>N$, which their detection procedure requires.

${ }^{16}$ The detection outcomes also very much depend on whether one uses the modification of the BM procedure or not. The results for unmodified BM is in the online supplement.
} 
last two columns of Table 7, for the full sample BM selects Fluid Milk as the dominant sector if observations are not standardized, and selects Automobiles and Light Duty Motor Vehicles, and Motor Vehicle Parts, as dominant when observations are standardized. For the two sub-samples the results are much more dispersed, and only Motor Vehicle Parts is included in the list of the dominant sectors for all sub-samples when the observations are standardized. Considering that by construction BM will end up with at least one sector as dominant, and the Monte Carlo evidence suggests that BM is particularly prone to false discovery when observations are standardized, the detection outcome of the BM procedure for this application should be approached with caution.

In addition to splitting the sample at the end of 1983, we also applied our detection method to rolling samples with window sizes of 10, 12, 15 and 20 years, in order to obtain further evidence on how the number and identity of dominant units could be subject to change. As previously, the maximum admissible number of common factors and dominant units is set to $p_{\max } \in\{2,3,4,5,6\}$. For the sake of brevity, only SMT $-\sigma^{2}$ thresholding is considered. The results unanimously confirm our previous finding that there is no dominant sector in the U.S. industrial production.

\subsection{Are there dominant economies or equity markets in the global economy?}

In a second application, we use quarterly observations on real GDP and real equity prices over a number of countries and equity markets spanning the period 1979Q2-2016Q4, providing $T=151$ observations for each country. ${ }^{17}$ Data on real GDP is available for 33 countries and account for over 90 percent of global output. The equity price observations are available for 26 countries, and include all major equity markets.

\subsubsection{Cross country output growths}

A recent investigation of cross country correlation of real GDP growth rates is given in CesaBianchi, Pesaran, and Rebucci (2018), and shows that accounting for one common factor is enough to reduce average pairwise cross country correlations to almost zero. Despite this suggestive evidence for the presence of only one factor in GDP, we consider a wider set of choices concerning the number of latent factors, and experiment with $p_{\max } \in\{2,3,4,5,6\}$ when applying $\sigma^{2}$ thresholding. As in the previous application, the results from the application of SMT- $\sigma^{2}$ thresholding are compared to the other two detection procedures (BM and BM standardized as well as PS). The results are summarized in Table 8. In this application SMT $-\sigma^{2}$ thresholding selects 1 country (France) as dominant in terms of GDP growth when $p_{\max }=3,4$ or 5 , and selects no dominant country if $p_{\max }=2$ or 6 . Given the cross country growth evidence provided by Cesa-Bianchi, Pesaran, and Rebucci (2018) it is more reasonable to rely on the detection evidence when $p_{\max }=2$, which is compatible with assuming one common global technology factor (i.e. $k_{0}=1$ ) with one possible dominant country, say U.S., (with $m_{0}=1$ ) which gives $p_{\max }=2$. Also if we use the $I C_{p 2}$ criterion of Bai and $\mathrm{Ng}$ (2002) to select the number of factors across country growth rates we also end up with one factor. (see footnote 1 of Table 8). So we conclude that there is no compelling evidence for the presence of a dominant

\footnotetext{
${ }^{17}$ Cross country data is taken from the latest vintage of the GVAR data set as described in Mohaddes and Raissi (2018).
} 


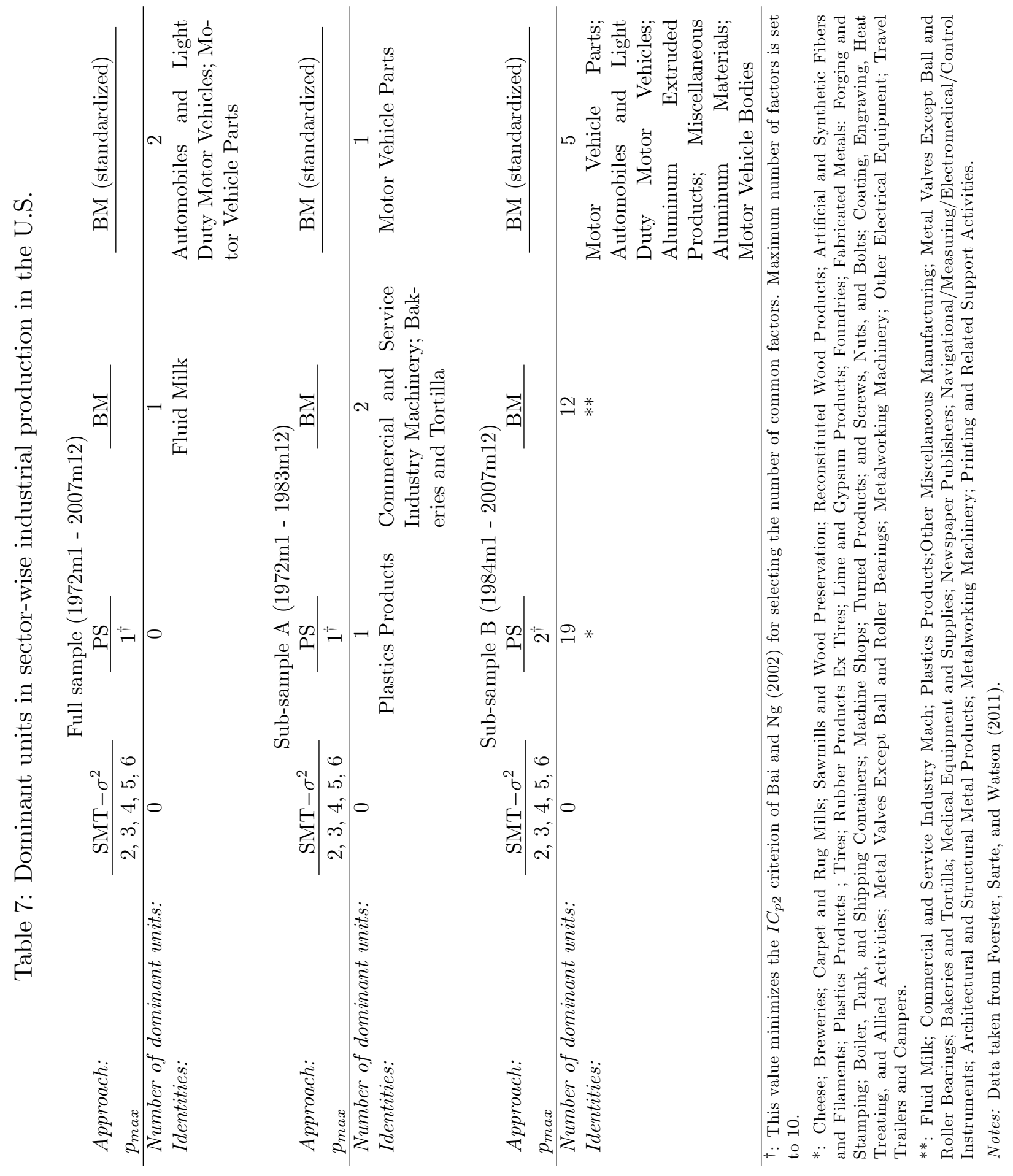


country in terms of output growth, and the detection of France as a dominant economy when $p_{\max }=3,4$ and 5, is most likely a false discovery. This conclusion is also supported when we consider the result obtained from the application of the PS procedure to the GDP growth series. In contrast, BM procedure selects France and Spain as dominant economies when the growth series are not standardized, and selects an additional 9 economies (a total of 11 economies out of 33) as dominant, if observations are standardized. This outcome is difficult to interpret and most likely reflects the tendency of the BM procedure to over-select as documented in the MC section.

Table 8: Dominant unit detection methods applied to cross country rates of change of real GDP (33 countries) and real equity prices (26 markets) over the period 1979Q2-2016Q4 (151 time periods)

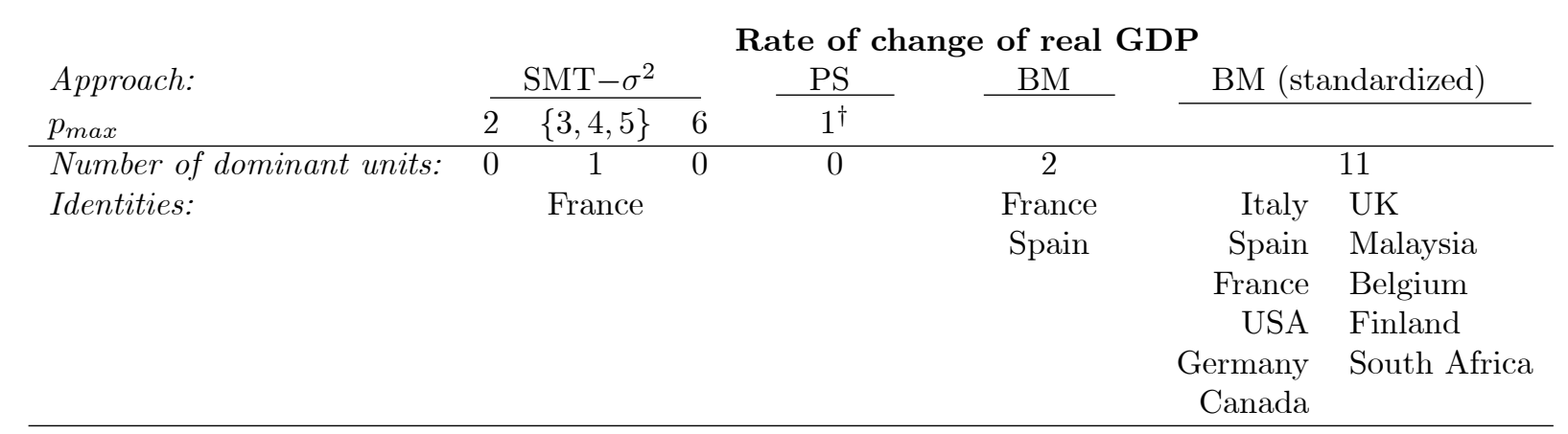

\begin{tabular}{|c|c|c|c|c|}
\hline \multirow[b]{2}{*}{ Approach: } & \multicolumn{4}{|c|}{ Rate of change of real equity prices } \\
\hline & $\mathrm{SMT}-\sigma^{2}$ & PS & $\mathrm{BM}$ & BM (standardized) \\
\hline$p_{\max }$ & $2,3,4,5,6$ & $2^{\dagger}$ & & \\
\hline Number of dominant units: & 0 & 6 & 6 & 1 \\
\hline Identities: & & France & USA & Netherlands \\
\hline & & Germany & Netherlands & \\
\hline & & Malaysia & UK & \\
\hline & & Netherlands & Canada & \\
\hline & & Singapore & Switzerland & \\
\hline & & Thailand & Germany & \\
\hline
\end{tabular}

\subsubsection{Cross market rate of change of real equity prices}

The results for the rate of change of real equity prices are summarized in the lower panel of Table 8. In this application SMT $-\sigma^{2}$ thresholding is the only method not identifying any of the equity markets as dominant. Both PS and BM procedures select 6 markets as dominant, and agree only on Germany and Netherlands as the dominant equity markets. Interestingly enough, BM only selects Netherlands as dominant when observations are standardized. Once again we find the BM detection method to be highly sensitive to standardization of observations.

Finally, it is important to bear in mind, that not finding a dominant unit does not mean that the global economy is not subject to global shocks. Our results suggest that once we allow for the possibility of global shocks, it is difficult to find convincing evidence that any country can be singled out as dominant. This result is also compatible with the presence of influential 
economies such as U.S., China, Japan and Germany as having important global and regional impacts in the world economy.

\subsection{U.S. house price changes}

It is well established that house price changes in the U.S. are governed by common national and regional factors (see e.g. Holly, Pesaran, and Yamagata, 2010; Bailey, Holly, and Pesaran, 2016), and it is of interest to investigate if any of these common factors are due to the dominance of particular states amongst the 48 mainland states of the U.S.. To this end we consider statelevel quarterly data on real house prices over the 1975Q1-2014Q4 period $(T=160) \cdot{ }^{18}$ In our analysis we use the rate of change of real house prices, after seasonal adjustment, with nominal house prices deflated by the state-level consumer price indices.

Table 9: Estimated U.S. states with dominant housing market

\begin{tabular}{|c|c|c|c|c|c|c|}
\hline Approach: & & $\mathrm{SMT}-\sigma^{2}$ & & PS & $\mathrm{BM}$ & BM (standardized) \\
\hline$p_{\max }$ & 2 & 3 & $4,5,6$ & $5^{\dagger}$ & & \\
\hline $\begin{array}{l}\text { Number of dom- } \\
\text { inant units: }\end{array}$ & 1 & 2 & 0 & 2 & 4 & 6 \\
\hline Identities: & New York & $\begin{array}{l}\text { Kentucky } \\
\text { New York }\end{array}$ & & $\begin{array}{c}\text { New Hampshire } \\
\text { Nevada }\end{array}$ & $\begin{array}{c}\text { North Carolina } \\
\text { Maryland } \\
\text { Virginia } \\
\text { Connecticut }\end{array}$ & $\begin{array}{c}\text { Connecticut } \\
\text { New Hampshire } \\
\text { Massachusetts } \\
\text { Maryland } \\
\text { Virginia } \\
\text { Rhode Island }\end{array}$ \\
\hline
\end{tabular}

$\dagger$ : This value minimizes the $I C_{p 2}$ criterion of Bai and $\mathrm{Ng}$ (2002) for selecting the number of common factors. Maximum number of factors is set to 10 .

Notes: Data taken from Freddie Mac House Price Indexes and Yang (2018).

To investigate whether house price changes in any of 48 mainland U.S. states could be regarded as dominant or pervasive for the rest of the states, as in the previous applications, we implement SMT $-\sigma^{2}$ thresholding with $p_{\max }=\{2,3,4,5,6\}$. The PS and BM methods are applied as before. The results are summarized in Table 9. As can be seen there are significant differences in the outcomes depending on the method used. In the case of SMT $-\sigma^{2}$ thresholding New York is identified as dominant when the maximum number of common factors is set to 2 and 3. No dominant unit is found for $p_{\max } \in\{4,5,6\}$, and Kentucky is also selected as dominant when $p_{\max }=3$, which could be false discovery. The BM procedure identifies many more states as dominant with no clear geographical patterns. Without standardization, BM selects North Carolina, Maryland, Virginia and Connecticut as dominant, whilst with standaridization three additional states are selected as dominant, namely New Hampshire, Massachusetts and Rhode Island. Connecticut is not selected when we use BM (standardized). We take these results as weak evidence for the influential role of the north-eastern part of the United States with New York being the most plausible candidate. By contrast, PS detects two dominant units in two opposite corners of the U.S., namely New Hampshire and Nevada, thus providing a less coherent picture compared to the other two approaches.

\footnotetext{
${ }^{18}$ House price data is taken from Freddie Mac House Price Indexes (http://www.freddiemac.com/research/ indices/house-price-index.html). State-level consumer price indexes were taken from Yang (2018) who updated a previously constructed dataset of Bailey, Holly, and Pesaran (2016).
} 


\section{Concluding remarks}

Recent developments in network and panel literature have emphasized the importance of some key units for interdependencies in networks. For example, financial networks can be resilient with no units playing an unduly important (i.e. 'systemic') role while others may have dominant units that need close monitoring. There is a small literature on how to detect such units but all existing methods are either not rigorously analyzed or have drawbacks such as assuming, rather that ascertaining, the presence of at least one dominant unit, or considering networks with a relatively small number of units in the network.

We contribute to this literature by proposing a new thresholding method which is rigorously developed using theory on large factor models as well as recent developments on multiple testing. It has good small sample properties and allows for the presence of no dominant units while being able to detect weakly influential cross-section entities. Furthermore, our method is versatile in that it can be applied for a wider combination of cross-sectional and time sample dimensions and that it is able to handle the presence of external common factors. 


\section{Appendix}

This appendix is in two parts. Part A provides the proof of the main results, whilst Part B states and proves a number of auxiliary lemmas needed in the main proofs.

\section{A Proof of main results}

\section{A.1 Proof of Theorem 1}

We need to show that

$$
\lim _{N, T \rightarrow \infty} \operatorname{Pr}\left(\left\{\hat{I}_{D}=I_{D}\right\} \cap\left\{\hat{I}_{N D}=I_{N D}\right\}\right)=1 .
$$

It suffices to show that

$$
\lim _{N, T \rightarrow \infty} \operatorname{Pr}\left(\cap_{i \in I_{D}}\left\{\hat{\sigma}_{i T}^{2} \leq \frac{2 \hat{\eta}_{i N}^{2} \log T}{N}\right\}\right)=1,
$$

and

$$
\lim _{N, T \rightarrow \infty} \operatorname{Pr}\left(\cup_{i \in I_{N D}}\left\{\hat{\sigma}_{i T}^{2} \leq \frac{2 \hat{\eta}_{i N}^{2} \log T}{N}\right\}\right)=0
$$

Let

$$
\eta_{i N}^{2}=\frac{\mathbf{a}_{i}^{\prime} \mathbf{A}_{0}^{\prime} \boldsymbol{\Sigma}_{v} \mathbf{A}_{0} \mathbf{a}_{i}}{N} \text {, and } C_{i N T}=\frac{2 \eta_{i N}^{2} \log T}{N} .
$$

Then, we need to show equivalently that

$$
\lim _{N, T \rightarrow \infty} \operatorname{Pr}\left(\cap_{i \in I_{D}}\left\{\hat{\sigma}_{i T}^{2}+\frac{2 \log T}{N}\left(\eta_{i N}^{2}-\hat{\eta}_{i N}^{2}\right) \leq C_{i N T}\right\}\right)=1,
$$

and

$$
\lim _{N, T \rightarrow \infty} \operatorname{Pr}\left(\cup_{i \in I_{N D}}\left\{\hat{\sigma}_{i T}^{2}+\frac{2 \log T}{N}\left(\eta_{i N}^{2}-\hat{\eta}_{i N}^{2}\right) \leq C_{i N T}\right\}\right)=0 .
$$

Proceeding from (28), if $i \in I_{D}$, we have $N \hat{\sigma}_{i T}^{2}=\sum_{j=1}^{6} B_{i j}$, where $B_{i j}$ are defined below equation (28), and

$$
B_{i j}=o_{p}(1), \text { for all } i, \text { and } j=2,3, \ldots, 6,
$$

as long as $\frac{\sqrt{N}}{T} \rightarrow 0$. If $i \in I_{N D}$ then $N \hat{\sigma}_{i T}^{2}=\sum_{j=1}^{8} B_{i j}$, where, recalling (35) and (36),

$$
B_{i 7}=\frac{N \mathbf{v}_{i}^{\prime} \mathbf{M}_{\hat{\mathbf{F}}} \mathbf{v}_{i}}{T}, \text { and } B_{i 8}=\frac{N \mathbf{a}_{i}^{\prime} \mathbf{F}_{0}^{\prime} \mathbf{M}_{\hat{\mathbf{F}}} \mathbf{v}_{i}}{T}=\frac{N \mathbf{a}_{i}^{\prime}\left(\mathbf{F}_{0}-\hat{\mathbf{F}}\right)^{\prime} \mathbf{M}_{\hat{\mathbf{F}}} \mathbf{v}_{i}}{T}
$$

It is straightforward to show that $B_{i 7}=O_{p}(N)$. Further, $B_{i 8}=O_{p}\left(\frac{N}{\min (\sqrt{N}, T)}\right)=o_{p}(N)$. A detailed analysis of $B_{i 7}$ and $B_{i 8}$ is provided in Section A.2. Terms $B_{i j}, j=1, \ldots, 6$ depend on $i$ only through $\mathbf{a}_{i}$ and it is assumed that $\sup _{i}\left\|\mathbf{a}_{i}\right\|^{2}<C<\infty$. Therefore, it follows immediately that

$$
\lim _{N, T \rightarrow \infty} \operatorname{Pr}\left(\sup _{i}\left|B_{i j}\right| \leq D_{N T}\right)=1, j=1,2, \ldots, 6,
$$


for any sequence $D_{N T}$ bounded away from zero. Further, we need to show that

$$
\lim _{N, T \rightarrow \infty} \operatorname{Pr}\left(\cap_{i \in I_{N D}}\left\{\left|B_{i 7}+B_{i 8}\right|>C_{i N T}\right\}\right)=1
$$

and it will be sufficient (assuming $N C_{i N T}=o\left(\min (\sqrt{N}, T)^{a}\right)$ ) to show that

$$
\lim _{N, T \rightarrow \infty} \sum_{i \in I_{N D}} \operatorname{Pr}\left(\left|B_{i 7}\right|<\min (\sqrt{N}, T)^{a}\right)=0,
$$

and

$$
\lim _{N, T \rightarrow \infty} \sum_{i \in I_{N D}} \operatorname{Pr}\left(\left|B_{i 8}\right|>\min (\sqrt{N}, T)^{a}\right)=0,
$$

for some $0<a<1$. This result follows straightforwardly by noting from a direct application of Lemma A7 of Chudik, Kapetanios, and Pesaran (2018) that

$$
\operatorname{Pr}\left(\left|\mathbf{v}_{i}^{\prime} \mathbf{M}_{\mathbf{F}} \mathbf{v}_{i}-T \sigma_{v_{i}}^{2}\right|>T N C_{N T}\right) \leq \exp \left(-C T N^{2} C_{N T}^{2}\right)=\exp \left[-C T \eta_{i N}^{4}(\log T)^{2}\right],
$$

for some $C>0$. It is easily seen that $N \exp \left(-C T \eta_{i N}^{4}(\log T)^{2}\right)=o(1)$, noting that $\sup _{i}\left(\eta_{i N}^{4}\right)>$ 0. A similar result obtains for $\operatorname{Pr}\left(\left|\mathbf{v}_{i}^{\prime} \mathbf{M}_{\hat{\mathbf{F}}} \mathbf{v}_{i}-\mathbf{v}_{i}^{\prime} \mathbf{M}_{\mathbf{F}} \mathbf{v}_{i}\right|>T N C_{N T}\right)$, along the lines of our analysis below for $\eta_{i N}$ starting with (55).

To complete the proof it now suffices to show that

$$
\lim _{N, T \rightarrow \infty} \operatorname{Pr}\left(\cap_{i=1,2, \ldots, N}\left\{\left|B_{i 1}\right| \leq N C_{i N T}\right\}\right)=1
$$

and

$$
\lim _{N, T \rightarrow \infty} \operatorname{Pr}\left(\cap_{i=1,2, \ldots, N}\left\{\left|\eta_{i N}^{2}-\hat{\eta}_{i N}^{2}\right| \leq \eta_{i N}^{2}\right\}\right)=1,
$$

or $\lim _{N, T \rightarrow \infty} \operatorname{Pr}\left(\sup _{i}\left|\eta_{i N}^{2}-\hat{\eta}_{i N}^{2}\right| \leq C\right)=1$, for some finite $C>0$, since $\eta_{i N}^{2}$ is uniformly bounded away from zero and infinity. (53) follows from auxiliary Lemmas 3-6.

Consider now (54), and note that by equations (40) and (45), we have

$$
\eta_{i N}^{2}-\hat{\eta}_{i N}^{2}=\frac{\mathbf{a}_{i}^{\prime} \mathbf{A}_{0}^{\prime} \boldsymbol{\Sigma}_{v} \mathbf{A}_{0} \mathbf{a}_{i}}{N}-\frac{\hat{\mathbf{a}}_{i}^{\prime} \hat{\mathbf{A}}^{\prime} \tilde{\boldsymbol{\Sigma}}_{v} \hat{\mathbf{A}} \hat{\mathbf{a}}_{i}}{N}
$$

Then,

$$
\begin{aligned}
\left|\eta_{i N}^{2}-\hat{\eta}_{i N}^{2}\right| & \leq \\
& C_{1}\left|\frac{\mathbf{a}_{i}^{\prime} \mathbf{A}_{0}^{\prime} \boldsymbol{\Sigma}_{v} \mathbf{A}_{0}\left(\mathbf{a}_{i}-\hat{\mathbf{a}}_{i}\right)}{N}\right|+C_{2}\left|\frac{\mathbf{a}_{i}^{\prime} \mathbf{A}_{0}^{\prime}\left(\tilde{\boldsymbol{\Sigma}}_{v}-\boldsymbol{\Sigma}_{v}\right) \mathbf{A}_{0} \mathbf{a}_{i}}{N}\right|+C_{3}\left|\frac{\mathbf{a}_{i}^{\prime} \mathbf{A}_{0}^{\prime} \boldsymbol{\Sigma}_{v}\left(\hat{\mathbf{A}}-\mathbf{A}_{0}\right) \mathbf{a}_{i}}{N}\right| \\
& =A_{i 1}+A_{i 2}+A_{i 3},
\end{aligned}
$$

where $A_{i 2}$ and $A_{i 3}$ depend on $i$ only via $\mathbf{a}_{i}$. By the boundedness of $\mathbf{a}_{i}$, auxiliary Lemmas $3-6$, and Theorem 1 of Bailey, Pesaran, and Smith (2018), $A_{i 2}=o_{p}(1)$ and $A_{i 3}=o_{p}(1)$. Hence

$$
\lim _{N, T \rightarrow \infty} \operatorname{Pr}\left(\sup _{i} A_{i 2} \leq C\right)=1, \text { and } \lim _{N, T \rightarrow \infty} \operatorname{Pr}\left(\sup _{i} A_{i 3} \leq C\right)=1 .
$$


Now consider $A_{i 1}$, and note that each element of $N^{-1} \mathbf{a}_{i}^{\prime} \mathbf{A}_{0}^{\prime} \boldsymbol{\Sigma}_{v} \mathbf{A}_{0}$ is uniformly bounded. Therefore, it suffices to show that $\lim _{N, T \rightarrow \infty} \operatorname{Pr}\left(\sup _{i}\left\|\mathbf{a}_{i}-\hat{\mathbf{a}}_{i}\right\| \leq C\right)=1$. We have

$$
\mathbf{a}_{i}-\hat{\mathbf{a}}_{i}=\frac{1}{T} \sum_{t=1}^{T} \mathbf{f}_{t} v_{i t}+\frac{1}{T} \sum_{t=1}^{T} x_{i t}\left(\hat{\mathbf{f}}_{t}-\mathbf{f}_{t}\right)
$$

So we need to show that

$$
\lim _{N, T \rightarrow \infty} \operatorname{Pr}\left(\sup _{i}\left\|\sum_{t=1}^{T} \mathbf{f}_{t} v_{i t}\right\| \leq T C\right)=1
$$

and

$$
\lim _{N, T \rightarrow \infty} \operatorname{Pr}\left[\sup _{i}\left\|\sum_{t=1}^{T} x_{i t}\left(\hat{\mathbf{f}}_{t}-\mathbf{f}_{t}\right)\right\| \leq T C\right]=1 .
$$

(56) follows easily. We focus on (57). For example, by (A1) of Bai (2003) we note that

$$
\hat{f}_{j t}-f_{j t}=\frac{1}{T} \sum_{l=1}^{T} \hat{f}_{j l} \gamma_{l t}+\frac{1}{T} \sum_{l=1}^{T} \hat{f}_{j l} \zeta_{l t}+\frac{1}{T} \sum_{l=1}^{T} \hat{f}_{j l} \varkappa_{l t}+\frac{1}{T} \sum_{l=1}^{T} \hat{f}_{j l} \xi_{l t}
$$

where $\gamma_{l t}=\gamma_{N, l t}=N^{-1} \sum_{i=1}^{N} E\left(v_{i t} v_{i l}\right), \zeta_{l t}=N^{-1} \mathbf{v}_{l}^{\prime} \mathbf{v}_{t}-\gamma_{l t}, \varkappa_{l t}=N^{-1} \mathbf{f}_{l}^{\prime} \mathbf{A}_{0}^{\prime} \mathbf{v}_{t}$, and $\xi_{l t}=\varkappa_{t l}$. So we need to show the following ( $C$ changes from instance to instance).

$$
\begin{aligned}
& \lim _{N, T \rightarrow \infty} \operatorname{Pr}\left[\sup _{i}\left|\sum_{t=1}^{T} x_{i t}\left(\frac{1}{T} \sum_{l=1}^{T} \hat{f}_{j l} \gamma_{l t}\right)\right| \leq T C\right]=1, \\
& \lim _{N, T \rightarrow \infty} \operatorname{Pr}\left[\sup _{i}\left|\sum_{t=1}^{T} x_{i t}\left(\frac{1}{T} \sum_{l=1}^{T} \hat{f}_{j l} \zeta_{l t}\right)\right| \leq T C\right]=1,
\end{aligned}
$$

and

$$
\lim _{N, T \rightarrow \infty} \operatorname{Pr}\left[\sup _{i}\left|\sum_{t=1}^{T} x_{i t}\left(\frac{1}{T} \sum_{l=1}^{T} \hat{f}_{j l} \varkappa_{l t}\right)\right| \leq T C\right]=1 .
$$

We proceed in turn.

$$
\begin{aligned}
\operatorname{Pr} & {\left[\sup _{i}\left|\sum_{t=1}^{T} x_{i t}\left(\frac{1}{T} \sum_{l=1}^{T} \hat{f}_{j l} \gamma_{l t}\right)\right|>T C\right] \leq \operatorname{Pr}\left[\sup _{i}\left|\frac{1}{T} \sum_{t=1}^{T} \sum_{l=1}^{T} x_{i t}\left(\hat{f}_{j l}-f_{j l}\right) \gamma_{l t}\right|>T C\right]+} \\
& \operatorname{Pr}\left(\sup _{i}\left|\frac{1}{T} \sum_{t=1}^{T} \sum_{l=1}^{T} x_{i t} f_{j l} \gamma_{l t}\right|>T C\right)=A_{11 i}+A_{12 i} .
\end{aligned}
$$


We have, for some $0<a<1$,

$$
\begin{aligned}
A_{11 i} & =\operatorname{Pr}\left(\sup _{i}\left|\frac{1}{T} \sum_{t=1}^{T} \sum_{l=1}^{T} x_{i t}\left(\hat{f}_{j l}-f_{j l}\right) \gamma_{l t}\right|>T C\right) \leq \\
& \operatorname{Pr}\left\{\begin{array}{l}
\left.\frac{1}{T^{1 / 2}}\left[\frac{\min (N, T)^{a}}{T} \sum_{l=1}^{T}\left(\hat{f}_{j l}-f_{j l}\right)^{2}\right]^{1 / 2}\left[\frac{1}{T} \sum_{t=1}^{T} \sum_{l=1}^{T} \gamma_{l t}^{2}\right]^{1 / 2}\right\} \\
\sup _{i}\left[\frac{\min (N, T)^{-a}}{T} \sum_{t=1}^{T} x_{i t}^{2}\right]^{1 / 2}>C
\end{array}\right\} \\
& \leq \operatorname{Pr}\left\{\frac{1}{T^{1 / 2}}\left[\frac{\min (N, T)^{a}}{T} \sum_{l=1}^{T}\left(\hat{f}_{j l}-f_{j l}\right)^{2}\right]^{1 / 2}\left[\frac{1}{T} \sum_{t=1}^{T} \sum_{l=1}^{T} \gamma_{l t}^{2}\right]^{1 / 2}>C\right\}+ \\
& \operatorname{Pr}\left[\sup _{i}\left(\frac{\min (N, T)^{-a}}{T} \sum_{t=1}^{T} x_{i t}^{2}\right)^{1 / 2}>C\right] .
\end{aligned}
$$

But using Theorem 1 in Bai and Ng (2002),

$$
\frac{1}{T^{1 / 2}}\left[\frac{\min (N, T)^{a}}{T} \sum_{l=1}^{T}\left(\hat{f}_{j l}-f_{j l}\right)^{2}\right]^{1 / 2}\left[\frac{1}{T} \sum_{t=1}^{T} \sum_{l=1}^{T} \gamma_{l t}^{2}\right]^{1 / 2}=o_{p}(1),
$$

and using Lemma 2 to show

$$
\operatorname{Pr}\left[\sup _{i}\left(\frac{\min (N, T)^{-a}}{T} \sum_{t=1}^{T} x_{i t}^{2}\right)^{1 / 2}>C\right]=o(1) .
$$

Hence, it follows that $A_{11 i}=o(1)$. Next

$$
\begin{aligned}
& A_{12 i}=\operatorname{Pr}\left(\sup _{i}\left|\frac{1}{T^{2}} \sum_{t=1}^{T} \sum_{l=1}^{T} x_{i t} f_{j l} \gamma_{l t}\right|>C\right) \leq \operatorname{Pr}\left(\left\|\frac{1}{T^{2}} \sum_{t=1}^{T} \sum_{l=1}^{T} \mathbf{f}_{t} f_{j l} \gamma_{l t}\right\|>C\right)+ \\
& \operatorname{Pr}\left(\sup _{i}\left|\frac{1}{T^{2}} \sum_{t=1}^{T} \sum_{l=1}^{T} v_{i t} f_{j l} \gamma_{l t}\right|>C\right) .
\end{aligned}
$$

But

$$
\operatorname{Pr}\left(\left\|\frac{1}{T^{2}} \sum_{t=1}^{T} \sum_{l=1}^{T} \mathbf{f}_{t} f_{j l} \gamma_{l t}\right\|>C\right)=o(1)
$$

We have

$$
\operatorname{Pr}\left[\sup _{i}\left|\frac{1}{T} \sum_{t=1}^{T} v_{i t}\left(\frac{1}{T} \sum_{l=1}^{T} f_{j l} \gamma_{l t}\right)\right|>C\right] .
$$

By the independence of $f_{j t}$ and $v_{i t}$ and the martingale difference (m.d.) property of $v_{i t}$, $\left(\frac{1}{T} \sum_{l=1}^{T} f_{j l} \gamma_{l t}\right) v_{i t}$ is also m.d., and by the martingale difference exponential inequality of Lemma A3 of Chudik, Kapetanios, and Pesaran (2018),

$$
\operatorname{Pr}\left[\sup _{i}\left|\frac{1}{T} \sum_{t=1}^{T} v_{i t}\left(\frac{1}{T} \sum_{l=1}^{T} f_{j l} \gamma_{l t}\right)\right|>C\right]=o(1) .
$$


Next, for $(60)$,

$$
\begin{aligned}
\operatorname{Pr} & {\left[\sup _{i}\left|\sum_{t=1}^{T} x_{i t}\left(\frac{1}{T} \sum_{l=1}^{T} \hat{f}_{j l} \zeta_{l t}\right)\right|>T C\right] \leq \operatorname{Pr}\left[\sup _{i}\left|\frac{1}{T} \sum_{t=1}^{T} \sum_{l=1}^{T} x_{i t}\left(\hat{f}_{j l}-f_{j l}\right) \zeta_{l t}\right|>T C\right]+} \\
& \operatorname{Pr}\left(\sup _{i}\left|\frac{1}{T} \sum_{t=1}^{T} \sum_{l=1}^{T} x_{i t} f_{j l} \zeta_{l t}\right|>T C\right)=A_{21 i}+A_{22 i} .
\end{aligned}
$$

But

$$
\begin{aligned}
& A_{21 i}=\operatorname{Pr}\left[\sup _{i}\left|\frac{1}{T^{2}} \sum_{t=1}^{T} \sum_{l=1}^{T} x_{i t}\left(\hat{f}_{j l}-f_{j l}\right) \zeta_{l t}\right|>C\right] \leq \\
& \operatorname{Pr}\left\{\left[\frac{\min (N, T)^{a}}{T} \sum_{l=1}^{T}\left(\hat{f}_{j l}-f_{j l}\right)^{2}\right]^{1 / 2} \sup _{i}\left[\frac{\min (N, T)^{-a}}{T^{2}} \sum_{t=1}^{T} \sum_{l=1}^{T} x_{i t}^{2} \zeta_{l t}^{2}\right]^{1 / 2}>C\right\} \leq \\
& \operatorname{Pr}\left\{\left[\frac{\min (N, T)^{a}}{T} \sum_{l=1}^{T}\left(\hat{f}_{j l}-f_{j l}\right)^{2}\right]^{1 / 2}>C\right\}+ \\
& \operatorname{Pr}\left\{\sup _{i}\left[\frac{\min (N, T)^{-a}}{T^{2}} \sum_{t=1}^{T} \sum_{l=1}^{T} x_{i t}^{2} \zeta_{l t}^{2}\right]>C\right\} .
\end{aligned}
$$

As before

$$
\operatorname{Pr}\left\{\left[\frac{\min (N, T)^{a}}{T} \sum_{l=1}^{T}\left(\hat{f}_{j l}-f_{j l}\right)^{2}\right]^{1 / 2}>C\right\}=o(1) .
$$

Then,

$$
\begin{aligned}
& \operatorname{Pr}\left\{\sup _{i}\left[\frac{\min (N, T)^{-a}}{T^{2}} \sum_{t=1}^{T} \sum_{l=1}^{T} x_{i t}^{2} \zeta_{l t}^{2}\right] \leq C\right\} \\
& \leq \operatorname{Pr}\left\{\sup _{i}\left[\left(\frac{\min (N, T)^{-2 a}}{T^{2}} \sum_{t=1}^{T} \sum_{l=1}^{T} x_{i t}^{4}\right)^{1 / 2}\left(\frac{1}{T^{2}} \sum_{t=1}^{T} \sum_{l=1}^{T} \zeta_{l t}^{4}\right)^{1 / 2}\right] \leq C\right\} \leq \\
& \operatorname{Pr}\left(\frac{1}{T^{2}} \sum_{t=1}^{T} \sum_{l=1}^{T} \zeta_{l t}^{4} \leq C\right)+ \\
& \operatorname{Pr}\left[\sup _{i}\left(\frac{1}{T} \sum_{t=1}^{T} x_{i t}^{4}\right) \leq C \min (N, T)^{2 a}\right] .
\end{aligned}
$$

But since $T^{-2} \sum_{t=1}^{T} \sum_{l=1}^{T} \zeta_{l t}^{4}=o_{p}(1)$, then $\operatorname{Pr}\left(T^{-2} \sum_{t=1}^{T} \sum_{l=1}^{T} \zeta_{l t}^{4}>C\right)=o(1)$, and using Lemma 2 we obtain

$$
\operatorname{Pr}\left[\sup _{i}\left(\frac{1}{T} \sum_{t=1}^{T} x_{i t}^{4}\right)>C \min (N, T)^{2 a}\right]=o(1) .
$$

A very similar analysis can be applied to (61), proving the required result. 


\section{A.2 Analyzing terms $B_{i 7}$ and $B_{i 8}$ for non dominant units}

We consider the terms $B_{i 7}$ and $B_{i 8}$, defined in (35) and (36), and wish to show that $B_{i 8}=$ $o_{p}\left(B_{i 7}\right)$. We note that

$$
B_{i 7}=\frac{N \mathbf{v}_{i}^{\prime} \mathbf{M}_{\hat{\mathbf{F}}} \mathbf{v}_{i}}{T}=N\left(\frac{\mathbf{v}_{i}^{\prime} \mathbf{M}_{F} \mathbf{v}_{i}}{T}\right)+\frac{N \mathbf{v}_{i}^{\prime}\left(\mathbf{M}_{\hat{\mathbf{F}}}-\mathbf{M}_{F}\right) \mathbf{v}_{i}}{T}
$$

and since $T^{-1} \mathbf{v}_{i}^{\prime} \mathbf{M}_{F} \mathbf{v}_{i}=O_{p}(1)$, so clearly $B_{i 7}=O_{p}(N)$. Consider now $B_{i 8}$, and note that by expression (27),

$$
\mathbf{M}_{\hat{\mathbf{F}}} \mathbf{F}_{0}=\frac{\mathbf{M}_{\hat{\mathbf{F}}}\left(\mathbf{F}_{0}-\hat{\mathbf{F}}\right) \mathbf{A}_{0}^{\prime}\left(\mathbf{A}_{0}-\hat{\mathbf{A}}\right)}{N}+\frac{\mathbf{M}_{\hat{\mathbf{F}}} \mathbf{V}\left(\mathbf{A}_{0}-\hat{\mathbf{A}}\right)}{N}-\frac{\mathbf{M}_{\hat{\mathbf{F}}} \mathbf{V A} \mathbf{A}_{0}}{N} .
$$

Hence

$$
\begin{aligned}
\mathbf{v}_{i}^{\prime} \mathbf{M}_{\hat{\mathbf{F}}} \mathbf{F}_{0} \mathbf{a}_{i} & =\frac{\mathbf{v}_{i}^{\prime} \mathbf{M}_{\hat{\mathbf{F}}}\left(\mathbf{F}_{0}-\hat{\mathbf{F}}\right) \mathbf{A}_{0}^{\prime}\left(\mathbf{A}_{0}-\hat{\mathbf{A}}\right) \mathbf{a}_{i}}{N} \\
& +\frac{\mathbf{v}_{i}^{\prime} \mathbf{M}_{\hat{\mathbf{F}}} \mathbf{V}\left(\mathbf{A}_{0}-\hat{\mathbf{A}}\right) \mathbf{a}_{i}}{N}-\frac{\mathbf{v}_{i}^{\prime} \mathbf{M}_{\hat{\mathbf{F}}} \mathbf{V} \mathbf{A}_{0} \mathbf{a}_{i}}{N} \\
& =\frac{B_{i 81}}{N}+\frac{B_{i 82}}{N}-\frac{B_{i 83}}{N}
\end{aligned}
$$

We examine $B_{i 81}, B_{i 82}$ and $B_{i 83}$. For $B_{i 81}$ we have

$$
\left|\mathbf{v}_{i}^{\prime} \mathbf{M}_{\hat{\mathbf{F}}}\left(\mathbf{F}_{0}-\hat{\mathbf{F}}\right) \mathbf{A}_{0}^{\prime}\left(\mathbf{A}_{0}-\hat{\mathbf{A}}\right) \mathbf{a}_{i}\right| \leq\left\|\mathbf{v}_{i}\right\|\left\|\mathbf{M}_{\hat{\mathbf{F}}}\right\|\left\|\mathbf{F}_{0}-\hat{\mathbf{F}}\right\|\left\|\mathbf{A}_{0}^{\prime}\left(\mathbf{A}_{0}-\hat{\mathbf{A}}\right)\right\|\left\|\mathbf{a}_{i}\right\| .
$$

Recall that by Assumption 2, $\left\|\mathbf{a}_{i}\right\|=O_{p}(1)$, whereas results (A) and (E) yield

$$
\left\|\mathbf{F}_{0}-\hat{\mathbf{F}}\right\|_{F}=O_{p}\left(\frac{\sqrt{T}}{\sqrt{\min (N, T)}}\right)
$$

and

$$
\left\|\mathbf{A}_{0}^{\prime}\left(\mathbf{A}_{0}-\hat{\mathbf{A}}\right)\right\|_{F}=O_{p}\left(\frac{N}{\sqrt{\min (N, T)}}\right) .
$$

Furthermore, since $\mathbf{M}_{\hat{\mathbf{F}}}$ is an idempotent matrix we also have $\left\|\mathbf{M}_{\hat{\mathbf{F}}}\right\|=O_{p}(1)$. Lastly, note that $\left\|\mathbf{v}_{i}\right\|=O_{p}(\sqrt{T})$ holds by Assumption 3 and the fact that $\mathbf{v}_{i}=\mathbf{u}_{i}$ for any non dominant unit, $i$. Consequently,

$$
\left|B_{i 81}\right|=\left|\mathbf{v}_{i}^{\prime} \mathbf{M}_{\hat{\mathbf{F}}}\left(\mathbf{F}_{0}-\hat{\mathbf{F}}\right) \mathbf{A}_{0}^{\prime}\left(\mathbf{A}_{0}-\hat{\mathbf{A}}\right) \mathbf{a}_{i}\right|=O_{p}\left(\frac{N T}{\min (N, T)}\right) .
$$

Next,

$$
\left|B_{i 82}\right|=\left|\mathbf{v}_{i}^{\prime} \mathbf{M}_{\hat{\mathbf{F}}} \mathbf{V}\left(\mathbf{A}_{0}-\hat{\mathbf{A}}\right) \mathbf{a}_{i}\right| \leq\left\|\mathbf{v}_{i}\right\|\left\|\mathbf{M}_{\hat{\mathbf{F}}}\right\|\left\|\mathbf{V}\left(\mathbf{A}_{0}-\hat{\mathbf{A}}\right)\right\|\left\|\mathbf{a}_{i}\right\| .
$$

Again, recall that by result $(\mathrm{C})$,

$$
\left\|\mathbf{V}\left(\mathbf{A}_{0}-\hat{\mathbf{A}}\right)\right\|_{F}=O_{p}\left(\frac{\sqrt{N T}}{\sqrt{\min (N, T)}}\right) .
$$


So

$$
\left|B_{i 82}\right|=\left|\mathbf{v}_{i}^{\prime} \mathbf{M}_{\hat{\mathbf{F}}} \mathbf{V}\left(\mathbf{A}_{0}-\hat{\mathbf{A}}\right) \mathbf{a}_{i}\right|=O_{p}\left(\frac{T \sqrt{N}}{\sqrt{\min (N, T)}}\right) .
$$

Next,

$$
\left|B_{i 83}\right|=\left|\mathbf{v}_{i}^{\prime} \mathbf{M}_{\hat{\mathbf{F}}} \mathbf{V} \mathbf{A}_{0} \mathbf{a}_{i}\right| \leq\left\|\mathbf{v}_{i}\right\|\left\|\mathbf{M}_{\hat{\mathbf{F}}}\right\|\left\|\mathbf{V} \mathbf{A}_{0}\right\|\left\|\mathbf{a}_{i}\right\|
$$

Here, result (D) yields $\left\|\mathbf{V A}_{0}\right\|_{F}=O_{p}(\sqrt{N T})$, and $\left|B_{i 83}\right|=O_{p}(\sqrt{N T})$. Overall,

$$
\begin{aligned}
B_{i 8} & =\frac{1}{T}\left(B_{i 81}+B_{i 82}-B_{i 83}\right) \\
& =O_{p}\left(\frac{N}{\min (N, T)}\right)+O_{p}\left(\frac{\sqrt{N}}{\sqrt{\min (N, T)}}\right)+O_{p}(\sqrt{N}) \\
& =o_{p}(N) .
\end{aligned}
$$

\section{A.3 Analysis of sequential $\sigma^{2}$ thresholding}

Consider the extension to a model of the form

$$
\begin{aligned}
& x_{i t}=\mathbf{a}_{i}^{\prime} \mathbf{f}_{t}+\mathbf{b}^{\prime} \mathbf{z}_{t}, \text { for } i=1,2, \ldots, m, \\
& x_{i t}=\mathbf{a}_{i}^{\prime} \mathbf{f}_{t}+\mathbf{b}^{\prime} \mathbf{z}_{t}+u_{i t}, \text { for } i=m+1, m+2, \ldots, N,
\end{aligned}
$$

where $\mathbf{z}_{t}$ is a known and observed vector of variables. We wish to repeat the analysis for $x_{i t}-\mathbf{b}^{\prime} \mathbf{z}_{t}$ but use OLS regression of $x_{i t}$ on $\mathbf{z}_{t}$ to obtain the OLS coefficient $\hat{\mathbf{b}}$ and construct $x_{i t}-\hat{\mathbf{b}}^{\prime} \mathbf{z}_{t}$. Repeating our earlier analysis without $\mathbf{z}_{t}$, we note that $N \hat{\sigma}_{i T}^{2}$ contains now a further term that potentially dominates other previously analyzed terms. This term is given by $\frac{N(\hat{\mathbf{b}}-\mathbf{b})^{\prime} \mathbf{Z}^{\prime} \mathbf{Z}(\hat{\mathbf{b}}-\mathbf{b})}{T}$. A possibility is to modify $N \hat{\sigma}_{i T}^{2}$ and consider $\min (N, T) \hat{\sigma}_{i T}^{2}$ instead. So we consider $(\hat{\mathbf{b}}-\mathbf{b})^{\prime} \mathbf{Z}^{\prime} \mathbf{Z}(\hat{\mathbf{b}}-\mathbf{b})$. We simplify the analysis by using a scalar $\mathbf{z}_{t}$. We wish to bound $\operatorname{Pr}\left[(\hat{\mathbf{b}}-\mathbf{b})^{\prime} \mathbf{Z}^{\prime} \mathbf{Z}(\hat{\mathbf{b}}-\mathbf{b})>C_{T}\right]$. We have

$$
\begin{aligned}
\operatorname{Pr}\left[(\hat{\mathbf{b}}-\mathbf{b})^{\prime} \mathbf{Z}^{\prime} \mathbf{Z}(\hat{\mathbf{b}}-\mathbf{b})>C_{T}\right] & =\operatorname{Pr}\left(\left|\left(\frac{\sum_{t=1}^{T} z_{t} v_{i t}}{\sum_{t=1}^{T} z_{t}^{2}}\right)^{2} \sum_{t=1}^{T} z_{t}^{2}\right|>C_{T}\right) \leq \\
& \operatorname{Pr}\left(\left|\left(\frac{\sum_{t=1}^{T} z_{t} v_{i t}}{\sum_{t=1}^{T} z_{t}^{2}}\right)^{2} \sum_{t=1}^{T} z_{t}^{2}\right|>C_{T}\right) .
\end{aligned}
$$

Using our derivations in the previous sections of the appendix, we have

$$
\begin{gathered}
\operatorname{Pr}\left(\left|\left(\frac{\sum_{t=1}^{T} z_{t} v_{i t}}{\sum_{t=1}^{T} z_{t}^{2}}\right)^{2} \sum_{t=1}^{T} z_{t}^{2}\right|>C_{T}\right) \leq \operatorname{Pr}\left(\left|\sum_{t=1}^{T} z_{t}^{2}-\sigma_{z}^{2}\right|>C / C_{T}\right)+ \\
\operatorname{Pr}\left(\left|\frac{1}{\sqrt{T}} \sum_{t=1}^{T} z_{t} v_{i t}\right|>C_{T}^{1 / 2}\right) .
\end{gathered}
$$


The right hand side of (62) can be bounded using a martingale difference exponential inequality, as before, thus providing justification for a criterion of the following form. Select unit $i$ to be dominant if

$$
\begin{aligned}
& \hat{\sigma}_{i T}^{2} \leq \frac{2 \hat{\eta}_{i N}^{2} \log T}{N}, \text { if } T \geq N \\
& \hat{\sigma}_{i T}^{2} \leq \frac{2 \hat{\sigma}_{i u}^{2} \log T}{T}, \text { if } T<N
\end{aligned}
$$

where $\hat{\sigma}_{i u}^{2}=\frac{1}{T} \sum_{t=1}^{T}\left(x_{i t}-\hat{\mathbf{b}}^{\prime} \mathbf{z}_{t}\right)^{2}$

\section{B Auxiliary Lemmas}

This section provides statements and proofs of the lemmas used in the paper. First we provide a lemma handling the remainder terms of $N \hat{\sigma}_{i T}^{2}$. We have

Lemma 1 Let $i$ denote a dominant unit, Assumptions 1-4 hold and $\frac{\sqrt{N}}{T} \rightarrow 0$. Then,

$$
N \hat{\sigma}_{i T}^{2}=\frac{\mathbf{a}_{i}^{\prime} \mathbf{A}_{0} \mathbf{V}^{\prime} \mathbf{M}_{\hat{\mathbf{F}}} \mathbf{V} \mathbf{A}_{0} \mathbf{a}_{i}}{N T}+O_{p}\left(\frac{1}{\delta_{N T}}\right)+O_{p}\left(\frac{\sqrt{N}}{\delta_{N T}^{2}}\right),
$$

where $\delta_{N T}^{2}=\min (N, T)$.

Proof. Since unit $i$ is dominant then using (28) we note that

$$
N \hat{\sigma}_{i T}^{2}=\frac{\mathbf{a}_{i}^{\prime} \mathbf{A}_{0} \mathbf{V}^{\prime} \mathbf{M}_{\hat{\mathbf{F}}} \mathbf{V} \mathbf{A}_{0} \mathbf{a}_{i}}{N T}+\sum_{j=2}^{6} B_{i j},
$$

where $B_{i j}$ for $j=2,3, \ldots, 6$ are given by (30)-(34) which we reproduce here for convenience

$$
\begin{aligned}
& B_{i 2}=2 \frac{\mathbf{a}_{i}^{\prime} \mathbf{A}_{0}^{\prime} \mathbf{V}^{\prime} \mathbf{M}_{\hat{\mathbf{F}}} \mathbf{V}\left(\mathbf{A}_{0}-\hat{\mathbf{A}}\right) \mathbf{a}_{i}}{N T}, \\
& B_{i 3}=2 \frac{\mathbf{a}_{i}^{\prime} \mathbf{A}_{0}^{\prime} \mathbf{V}^{\prime} \mathbf{M}_{\hat{\mathbf{F}}}\left(\mathbf{F}_{0}-\hat{\mathbf{F}}\right) \mathbf{A}_{0}^{\prime}\left(\mathbf{A}_{0}-\hat{\mathbf{A}}\right) \mathbf{a}_{i}}{N T}, \\
& B_{i 4}=\frac{\mathbf{a}_{i}^{\prime}\left(\mathbf{A}_{\mathbf{0}}-\hat{\mathbf{A}}\right)^{\prime} \mathbf{V}^{\prime} \mathbf{M}_{\hat{\mathbf{F}}} \mathbf{V}\left(\mathbf{A}_{0}-\hat{\mathbf{A}}\right) \mathbf{a}_{i}}{N T}, \\
& B_{i 5}=2 \frac{\mathbf{a}_{i}^{\prime}\left(\mathbf{A}_{\mathbf{0}}-\hat{\mathbf{A}}\right)^{\prime} \mathbf{V}^{\prime} \mathbf{M}_{\hat{\mathbf{F}}}\left(\mathbf{F}_{0}-\hat{\mathbf{F}}\right) \mathbf{A}_{0}^{\prime}\left(\mathbf{A}_{0}-\hat{\mathbf{A}}\right) \mathbf{a}_{i}}{N T}, \\
& B_{i 6}=\frac{\mathbf{a}_{i}^{\prime}\left(\mathbf{A}_{\mathbf{0}}-\hat{\mathbf{A}}\right)^{\prime} \mathbf{A}_{0}\left(\mathbf{F}_{0}-\hat{\mathbf{F}}\right)^{\prime} \mathbf{M}_{\hat{\mathbf{F}}}\left(\mathbf{F}_{0}-\hat{\mathbf{F}}\right) \mathbf{A}_{0}^{\prime}\left(\mathbf{A}_{0}-\hat{\mathbf{A}}\right) \mathbf{a}_{i}}{N T} .
\end{aligned}
$$

First, note that

$$
\left\|B_{i 2}\right\| \leq \frac{2}{N T}\left\|\mathbf{a}_{i}\right\|^{2}\left\|\mathbf{A}_{0}^{\prime} \mathbf{V}^{\prime}\right\|\left\|\mathbf{M}_{\hat{\mathbf{F}}}\right\|\left\|\mathbf{V}\left(\mathbf{A}_{\mathbf{0}}-\hat{\mathbf{A}}\right)\right\|
$$


But $\left\|\mathbf{M}_{\hat{\mathbf{F}}}\right\|=1$, since $\mathbf{M}_{\hat{\mathbf{F}}}$ is an idempotent matrix. Furthermore, $\sup _{i}\left\|\mathbf{a}_{i}\right\|^{2}<C$, by Assumption 2. Together with (C) and (D) of Proposition 1, these two results imply

$$
\left\|B_{i 2}\right\|=\frac{1}{N T} O_{p}(\sqrt{N T}) O_{p}\left(\frac{\sqrt{N T}}{\delta_{N T}}\right)=O_{p}\left(\frac{1}{\delta_{N T}}\right) .
$$

Similarly, using (A), (D) and (E) of Proposition 1,

$$
\begin{aligned}
\left\|B_{i 3}\right\| & \leq \frac{C}{N T}\left\|\mathbf{A}_{0}^{\prime} \mathbf{V}^{\prime}\right\|\left\|\mathbf{F}_{0}-\hat{\mathbf{F}}\right\|\left\|\mathbf{A}_{0}^{\prime}\left(\mathbf{A}_{\mathbf{0}}-\hat{\mathbf{A}}\right)\right\| \\
& =\frac{1}{N T} O_{p}(\sqrt{N T}) O_{p}\left(\frac{\sqrt{T}}{\delta_{N T}}\right) O_{p}\left(\frac{N}{\delta_{N T}}\right) \\
& =O_{p}\left(\frac{\sqrt{N}}{\delta_{N T}^{2}}\right)
\end{aligned}
$$

Next,

$$
\left\|B_{i 4}\right\| \leq \frac{C}{N T}\left\|\mathbf{V}\left(\mathbf{A}_{\mathbf{0}}-\hat{\mathbf{A}}\right)\right\|^{2}=O_{p}\left(\frac{1}{\delta_{N T}^{2}}\right),
$$

follows from (C). Using this latter result, as well as (A) and (E), we also obtain

$$
\begin{aligned}
\left\|B_{i 5}\right\| & \leq \frac{C}{N T}\left\|\mathbf{V}\left(\mathbf{A}_{\mathbf{0}}-\hat{\mathbf{A}}\right)\right\|\left\|\mathbf{F}_{0}-\hat{\mathbf{F}}\right\|\left\|\mathbf{A}_{0}^{\prime}\left(\mathbf{A}_{\mathbf{0}}-\hat{\mathbf{A}}\right)\right\| \\
& =\frac{1}{N T} O_{p}\left(\frac{\sqrt{N T}}{\delta_{N T}}\right) O_{p}\left(\frac{\sqrt{T}}{\delta_{N T}}\right) O_{p}\left(\frac{N}{\delta_{N T}}\right) \\
& =O_{p}\left(\frac{\sqrt{N}}{\delta_{N T}^{3}}\right) .
\end{aligned}
$$

Finally,

$$
\begin{aligned}
\left\|B_{i 6}\right\| & \leq \frac{C}{N T}\left\|\mathbf{A}_{0}^{\prime}\left(\mathbf{A}_{\mathbf{0}}-\hat{\mathbf{A}}\right)\right\|^{2}\left\|\mathbf{F}_{0}-\hat{\mathbf{F}}\right\|^{2} \\
& =\frac{1}{N T} O_{p}\left(\frac{N^{2}}{\delta_{N T}^{2}}\right) O_{p}\left(\frac{T}{\delta_{N T}^{2}}\right)=O_{p}\left(\frac{N}{\delta_{N T}^{4}}\right),
\end{aligned}
$$

by the same intermediate results. Summarizing the order results above and noting that $\frac{\sqrt{N}}{T} \rightarrow 0$, we have

$$
N \hat{\sigma}_{i T}^{2}=\frac{\mathbf{a}_{i}^{\prime} \mathbf{A}_{0}^{\prime} \mathbf{V}^{\prime} \mathbf{M}_{\hat{\mathbf{F}}} \mathbf{V} \mathbf{A}_{0} \mathbf{a}_{i}}{N T}+O_{p}\left(\frac{1}{\delta_{N T}}\right)+O_{p}\left(\frac{\sqrt{N}}{\delta_{N T}^{2}}\right),
$$

proving the required result.

Lemma 2 Let Assumptions 1-4 hold. Then,

$$
\operatorname{Pr}\left[\sup _{i}\left(\frac{1}{T} \sum_{t=1}^{T} x_{i t}^{j}\right)>C\right]=o(1), j=1,2,3,4 .
$$


Proof. We will prove the case for $j=4$ only. The cases for $j=1,2,3$ follow straightforwardly. We have

$$
x_{i t}=\mathbf{a}_{i}^{\prime} \mathbf{f}_{t}+v_{i t}=\varphi_{i t}+v_{i t}
$$

So

$$
x_{i t}^{4}=\varphi_{i t}^{4}+4 \varphi_{i t}^{3} v_{i t}+6 \varphi_{i t}^{2} v_{i t}^{2}+4 \varphi_{i t} v_{i t}^{3}+v_{i t}^{4}=\sum_{j}^{5} A_{j i t} .
$$

So

$$
\begin{aligned}
\operatorname{Pr}\left[\sup _{i}\left(\frac{1}{T} \sum_{t=1}^{T} x_{i t}^{4}\right)>C\right] & =\operatorname{Pr}\left[\sup _{i}\left[\sum_{j=1}^{5}\left(\frac{1}{T} \sum_{t=1}^{T} A_{j i t}\right)\right]>C\right] \\
& \leq \operatorname{Pr}\left[\sum_{j=1}^{5} \sup _{i}\left(\frac{1}{T} \sum_{t=1}^{T} A_{j i t}\right)>C\right] \\
& \leq \sum_{j=1}^{5} \operatorname{Pr}\left[\sup _{i}\left(\frac{1}{T} \sum_{t=1}^{T} A_{i j t}\right)>\pi_{j} C\right]=\sum_{j=1}^{5} B_{j},
\end{aligned}
$$

where $\pi_{j}>0$, and $\sum_{j=1}^{5} \pi_{j}=1$. We examine each $B_{j}$ in turn. We have that for sufficiently large finite constant $C$, there exists some constant $C_{1}$ such that

$$
\begin{aligned}
\operatorname{Pr}\left[\sup _{i}\left(\frac{1}{T} \sum_{t=1}^{T} A_{i 1 t}\right)>\pi_{1} C\right] & \leq \operatorname{Pr}\left[\sup _{i}\left|\frac{1}{T} \sum_{t=1}^{T}\left(\mathbf{a}_{i}^{\prime} \mathbf{f}_{t}\right)^{4}\right|>\pi_{1} C\right] \\
& =\operatorname{Pr}\left[\left(\sup _{i}\left\|\mathbf{a}_{i}\right\|^{4}\right)\left|\frac{1}{T} \sum_{t=1}^{T}\left\|\mathbf{f}_{t}\right\|^{4}\right|>\pi_{1} C\right] \\
& \leq \operatorname{Pr}\left[\mid \sum_{t=1}^{T}\left[\left\|\mathbf{f}_{t}\right\|^{4}-E\left(\left\|\mathbf{f}_{t}\right\|^{4}\right)\right]>T \pi_{1} C_{1}\right]
\end{aligned}
$$

However, since by Assumption $1, \frac{1}{T} \sum_{t=1}^{T}\left[\left\|\mathbf{f}_{t}\right\|^{4}-E\left(\left\|\mathbf{f}_{t}\right\|^{4}\right)\right]=o_{p}(1)$,

$$
\operatorname{Pr}\left[\left|\sum_{t=1}^{T}\left\|\mathbf{f}_{t}\right\|^{4}-E\left(\left\|\mathbf{f}_{t}\right\|^{4}\right)\right|>T \pi_{1} C_{1}\right]=o(1)
$$

for any finite $C_{1}>0$. For $B_{2}-B_{5}$ it is sufficient to note that $A_{j i t}, j=2, \ldots, 5$ are martingale difference processes since $\varphi_{i t}$ and $v_{i t}$ are independent and $v_{i t}^{j}-E\left(v_{i t}^{j}\right)$, for $j=1,2,3,4$ are martingale difference processes by the serial independence of $\varepsilon_{t}$ (see Assumption 3). Therefore, by the martingale difference exponential inequality Lemma A3 of Chudik, Kapetanios, and Pesaran (2018), we have that for $j=1, \ldots, 4$, and for a sufficiently large finite constant, $C$, there exist some constants $C_{1}$ and $C_{2}$ such that

$$
\operatorname{Pr}\left[\sup _{i}\left(\frac{1}{T} \sum_{t=1}^{T} A_{i j t}\right)>\pi_{j} C\right] \leq \operatorname{Pr}\left[\sup _{i}\left|\frac{1}{T} \sum_{t=1}^{T} A_{i j t}-E\left(A_{i j t}\right)\right|>\pi_{j} C_{1}\right] \leq \exp \left(-C_{2} T\right) \text {, }
$$

proving the result. 
The rest of the lemmas in this section prove the results of Proposition 1 in the main text. The five results $\mathrm{A}-\mathrm{E}$ are analysed in separate lemmas due to the length of the proofs. It is also important to note that the required assumptions for the subsequent lemmas are considerably weaker than those needed for consistency of the $\sigma^{2}$ thresholding procedure. The minimal conditions needed, which are satisfied by Assumptions 1-4 in the main text, as noted in Remark 2, are as follows:

1. $E\left\|\mathbf{f}_{t}\right\|^{4} \leq C<\infty, T^{-1} \sum_{t=1}^{T} \mathbf{f}_{t} \mathbf{f}_{t}^{\prime} \stackrel{p}{\rightarrow} \Sigma_{f}$ for some $m \times m$ positive definite matrix $\boldsymbol{\Sigma}_{f}$. $\mathbf{A}_{0}$ has bounded elements. Further $\left\|N^{-1} \mathbf{A}_{0}^{\prime} \mathbf{A}_{0}-\mathbf{D}\right\| \rightarrow 0$, as $N \rightarrow \infty$, where $\mathbf{D}$ is a positive definite matrix.

2. $E\left(v_{i t}\right)=0, E\left|v_{i t}\right|^{8} \leq C$ where $\mathbf{v}_{t}=\left(v_{1 t}, \ldots, v_{N t}\right)^{\prime}$ The variance of $\mathbf{v}_{t}$ is denoted by $\boldsymbol{\Sigma}_{v}$. $\mathbf{f}_{s}$ and $\mathbf{v}_{t}$ are independent for all $s, t$.

3. For $\tau_{i, j, t, s} \equiv E\left(v_{i t} v_{j s}\right)$ the following hold

- $(N T)^{-1} \sum_{s=1}^{T} \sum_{t=1}^{T}\left|\sum_{i=1}^{N} \tau_{i, i, t, s}\right| \leq C$.

- $\sum_{l=1}^{T}\left|1 / N \sum_{i=1}^{N} \tau_{i, i, s, l}\right| \leq C$ for all $s$.

- $N^{-1} \sum_{i=1}^{N} \sum_{j=1}^{N}\left|\tau_{i, j, s, s}\right| \leq C$.

- $(N T)^{-1} \sum_{s=1}^{T} \sum_{t=1}^{T} \sum_{i=1}^{N} \sum_{j=1}^{N}\left|\tau_{i, j, t, s}\right| \leq C$.

- For every $(t, s), E\left|(N)^{-1 / 2} \sum_{i=1}^{N}\left(v_{i s} v_{i t}-\tau_{i, i, s, t}\right)\right|^{4} \leq C$.

- For each $t, \frac{1}{\sqrt{N}} \sum_{i=1}^{N} \mathbf{a}_{i} v_{i t} \rightarrow^{d} N\left(0, \boldsymbol{\Gamma}_{t}\right)$ where $\boldsymbol{\Gamma}_{t}=\lim _{N \rightarrow \infty} \sum_{i=1}^{N} \sum_{j=1}^{N} E\left(\mathbf{a}_{i} \mathbf{a}_{j}^{\prime} v_{i t} v_{j t}\right)$.

The above list is essentially the set of assumptions in Bai (2003). Analogous to the definition in Section A.1, let $\gamma_{s t}=\gamma_{N, s t}=\frac{1}{N} \sum_{i=1}^{N} \tau_{i, i, t, s}$.

Lemma 3 Under Assumptions 1-4

$$
\left\|\frac{\mathbf{V}\left(\hat{\mathbf{A}}-\mathbf{A}_{0}\right)}{N}\right\|_{F}=O_{p}\left(\frac{\sqrt{T}}{\sqrt{N \min (N, T)}}\right)=O_{p}\left(\frac{\sqrt{T}}{N}\right)+O_{p}\left(\frac{1}{\sqrt{N}}\right) .
$$

Proof.

We have by the proof of Theorem 2 of Bai (2003, expression above (B.2)) that

$$
\hat{\mathbf{a}}_{i}-\mathbf{a}_{i}=\frac{1}{T} \sum_{t=1}^{T} \mathbf{f}_{t} v_{i t}+\frac{1}{T} \sum_{t=1}^{T} x_{i t}\left(\hat{\mathbf{f}}_{t}-\mathbf{f}_{t}\right) .
$$


We have

$$
\begin{aligned}
& \frac{1}{N^{2}}\left\|\mathbf{V}\left(\hat{\mathbf{A}}-\mathbf{A}_{0}\right)\right\|_{F}^{2}=\frac{1}{N^{2}} \sum_{t=1}^{T} \sum_{i=1}^{N} v_{i}^{2}\left(\hat{\mathbf{a}}_{i}-\mathbf{a}_{i}\right)^{\prime}\left(\hat{\mathbf{a}}_{i}-\mathbf{a}_{i}\right) \\
& \leq \frac{1}{N^{2}} \sum_{t=1}^{T} \sum_{i=1}^{N} v_{i t}^{2}\left(\frac{1}{T^{2}} \sum_{s=1}^{T} \mathbf{f}_{s}^{\prime} \mathbf{f}_{s} v_{i s}^{2}+\frac{1}{T^{2}} \sum_{s=1}^{T} \sum_{s^{\prime}, s \neq s^{\prime}}^{T} \mathbf{f}_{s}^{\prime} \mathbf{f}_{s^{\prime}} v_{i s} v_{i s^{\prime}}\right)+ \\
& \frac{1}{N^{2}} \sum_{t=1}^{T} \sum_{i=1}^{N} v_{i t}^{2}\left(\begin{array}{c}
\frac{1}{T^{2}} \sum_{s=1}^{T} x_{i s}^{2}\left\|\hat{\mathbf{f}}_{s}-\mathbf{f}_{s}\right\|_{F}^{2}+ \\
\frac{1}{T^{2}} \sum_{s=1}^{T} \sum_{s^{\prime}, s \neq s^{\prime}}^{T} \mid x_{i s} x_{i s^{\prime}}\left\|\hat{\mathbf{f}}_{s}-\mathbf{f}_{s}\right\|_{F}\left\|\hat{\mathbf{f}}_{s^{\prime}}-\mathbf{f}_{s^{\prime}}\right\|_{F}
\end{array}\right)+ \\
& \frac{1}{N^{2}} \sum_{t=1}^{T} \sum_{i=1}^{N} v_{i t}^{2}\left[\frac{1}{T^{2}} \sum_{s=1}^{T} \sum_{s^{\prime}=1}^{T} x_{i s^{\prime}} v_{i s} \mathbf{f}_{s}^{\prime}\left(\hat{\mathbf{f}}_{s^{\prime}}-\mathbf{f}_{s^{\prime}}\right)\right]= \\
& \frac{1}{N^{2}} \sum_{t=1}^{T} \sum_{i=1}^{N} v_{i t}^{2}\left(\frac{1}{T^{2}} \sum_{s=1}^{T} \mathbf{f}_{s}^{\prime} \mathbf{f}_{s} v_{i s}^{2}\right)+ \\
& \frac{1}{N^{2}} \sum_{t=1}^{T} \sum_{i=1}^{N} v_{i t}^{2}\left(\frac{1}{T^{2}} \sum_{s=1}^{T} \sum_{s^{\prime}, s \neq s^{\prime}}^{T} \mathbf{f}_{s}^{\prime} \mathbf{f}_{s^{\prime}} v_{i s} v_{i s^{\prime}}\right)+ \\
& \frac{1}{N^{2}} \sum_{t=1}^{T} \sum_{i=1}^{N} v_{i t}^{2}\left(\frac{1}{T^{2}} \sum_{s=1}^{T} x_{i s}^{2}\left\|\hat{\mathbf{f}}_{s}-\mathbf{f}_{s}\right\|_{F}^{2}\right)+ \\
& \frac{1}{N^{2}} \sum_{t=1}^{T} \sum_{i=1}^{N} v_{i t}^{2}\left(\frac{1}{T^{2}} \sum_{s=1}^{T} \sum_{s^{\prime}, s \neq s^{\prime}}^{T}\left|x_{i s} x_{i s^{\prime}}\right|\left\|\hat{\mathbf{f}}_{s}-\mathbf{f}_{s}\right\|_{F}\left\|\hat{\mathbf{f}}_{s^{\prime}}-\mathbf{f}_{s^{\prime}}\right\|_{F}\right)+ \\
& \frac{1}{N^{2}} \sum_{t=1}^{T} \sum_{i=1}^{N} v_{i t}^{2}\left[\frac{1}{T^{2}} \sum_{s=1}^{T} \sum_{s^{\prime}=1}^{T} x_{i s^{\prime}} v_{i s} \mathbf{f}_{s}^{\prime}\left(\hat{\mathbf{f}}_{s^{\prime}}-\mathbf{f}_{s^{\prime}}\right)\right] \\
& =\sum_{i=1}^{5} C_{i}
\end{aligned}
$$

We have

$$
C_{1}=\frac{1}{N^{2}} \sum_{t=1}^{T} \sum_{i=1}^{N} v_{i t}^{2}\left(\frac{1}{T} \sum_{s=1}^{T} \mathbf{f}_{s}^{\prime} \mathbf{f}_{s} v_{i s}^{2}\right)=\frac{1}{N^{2}} \sum_{i=1}^{N}\left(\frac{1}{T} \sum_{s=1}^{T} \mathbf{f}_{s}^{\prime} \mathbf{f}_{s} v_{i s}^{2}\right)\left(\frac{1}{T} \sum_{t=1}^{T} v_{i t}^{2}\right)=O_{p}\left(N^{-1}\right) .
$$

Also

$$
\begin{aligned}
C_{2} & =\frac{1}{N^{2}} \sum_{t=1}^{T} \sum_{i=1}^{N} v_{i t}^{2}\left(\frac{1}{T^{2}} \sum_{s=1}^{T} \sum_{s^{\prime}, s \neq s^{\prime}}^{T} \mathbf{f}_{s}^{\prime} \mathbf{f}_{s^{\prime}} v_{i s} v_{i s^{\prime}}\right) \\
& =\frac{1}{N^{2}} \frac{1}{T} \sum_{t=1}^{T} \sum_{i=1}^{N} v_{i t}^{2}\left[\frac{1}{\sqrt{T}} \sum_{s=1}^{T} v_{i s} \mathbf{f}_{s}^{\prime}\left(\frac{1}{\sqrt{T}} \sum_{s^{\prime}, s \neq s^{\prime}}^{T} \mathbf{f}_{s^{\prime}} v_{i s^{\prime}}\right)\right]=O_{p}\left(N^{-1}\right)
\end{aligned}
$$


Next, we have

$$
\begin{aligned}
C_{3} & =\frac{1}{T^{2} N^{2}} \sum_{t=1}^{T} \sum_{i=1}^{N} v_{i t}^{2}\left(\sum_{s=1}^{T} x_{i s}^{2}\left\|\hat{\mathbf{f}}_{s}-\mathbf{f}_{s}\right\|_{F}^{2}\right) \\
& =\sum_{j=1}^{m}\left[\frac{1}{T^{2} N^{2}} \sum_{t=1}^{T} \sum_{i=1}^{N} v_{i t}^{2}\left(\sum_{s=1}^{T} x_{i s}^{2}\left(\hat{f}_{j s}-f_{j s}\right)^{2}\right)\right] \leq \\
& \max _{j} \frac{1}{T^{2} N^{2}} \sum_{t=1}^{T} \sum_{i=1}^{N} v_{i t}^{2}\left(\sum_{s=1}^{T} x_{i s}^{2}\left(\hat{f}_{j s}-f_{j s}\right)^{2}\right) .
\end{aligned}
$$

But

$$
\begin{gathered}
\frac{1}{T^{2} N^{2}} \sum_{t=1}^{T} \sum_{i=1}^{N} v_{i t}^{2}\left(\sum_{s=1}^{T} x_{i s}^{2}\left(\hat{f}_{j s}-f_{j s}\right)^{2}\right)= \\
\frac{1}{T N^{2}} \sum_{s=1}^{T} \sum_{i=1}^{N}\left(\frac{1}{T} \sum_{t=1}^{T} v_{i t}^{2}\right) x_{i s}^{2}\left(\hat{f}_{j s}-f_{j s}\right)^{2}=\frac{1}{T N^{2}} \sum_{s=1}^{T} \sum_{i=1}^{N} z_{i s}\left(\hat{f}_{j s}-f_{j s}\right)^{2},
\end{gathered}
$$

where $z_{i t}=\left(\frac{1}{T} \sum_{s=1}^{T} v_{i s}^{2}\right) x_{i t}^{2}$. Note that $\sup _{i, t} E\left(z_{i t}^{2}\right)<\infty$. Then, by a similar analysis to term $A_{1}$ in (64) of Lemma 5,

$$
C_{3}=O_{p}\left(N^{-1} \min (N, T)^{-1}\right)
$$

Further,

$$
\begin{aligned}
C_{4} & =\frac{1}{N^{2}} \sum_{t=1}^{T} \sum_{i=1}^{N} v_{i t}^{2}\left(\frac{1}{T^{2}} \sum_{s=1}^{T} \sum_{s^{\prime}, s \neq s^{\prime}}^{T}\left|x_{i s} x_{i s^{\prime}}\right|\left\|\hat{\mathbf{f}}_{s}-\mathbf{f}_{s}\right\|_{F}\left\|\hat{\mathbf{f}}_{s^{\prime}}-\mathbf{f}_{s^{\prime}}\right\|_{F}\right) \\
& \leq \frac{1}{N^{2}} \sum_{t=1}^{T} \sum_{i=1}^{N} v_{i t}^{2}\left[\left(\frac{1}{T^{2}} \sum_{s=1}^{T} \sum_{s^{\prime}, s \neq s^{\prime}}^{T}\left(x_{i s} x_{i s^{\prime}}\right)^{2}\right)^{1 / 2}\right. \\
& \left.\left.\leq \frac{1}{N^{2}} \sum_{t=1}^{T} \sum_{i=1}^{T} \sum_{s^{\prime}, s \neq s^{\prime}}^{T}\left\|\hat{\mathbf{f}}_{s}-\mathbf{f}_{s}\right\|_{F}^{2}\left\|\hat{\mathbf{f}}_{s^{\prime}}-\mathbf{f}_{s^{\prime}}\right\|_{F}^{2}\right)^{1 / 2}\right] \\
& \left.\leq\left(\frac{1}{T} \sum_{s=1}^{T}\left\|\hat{\mathbf{f}}_{s}-\mathbf{f}_{s}\right\|_{F}^{2} \sum_{s}^{T}\left[\left\|\hat{\mathbf{f}}_{s}-\mathbf{f}_{s}\right\|_{F}^{2}\left(\frac{1}{T} \sum_{s^{\prime}, s \neq s^{\prime}}^{T}\left\|\hat{\mathbf{f}}_{s}-\mathbf{f}_{s}\right\|_{F}^{2}\right)\right]\right\}^{1 / 2}\right] \\
& \left\{\frac{1}{N^{2}} \sum_{t=1}^{T} \sum_{i=1}^{N} v_{i t}^{2}\left[\left(\frac{1}{T^{2}} \sum_{s=1}^{T} \sum_{s^{\prime}, s \neq s^{\prime}}^{T}\left|x_{i s} x_{i s^{\prime}}\right|\right)^{1 / 2}\right]\right\}
\end{aligned}
$$

But

$$
\sup _{i} E\left(\frac{1}{T^{2}} \sum_{s=1}^{T} \sum_{s^{\prime}, s \neq s^{\prime}}^{T}\left(x_{i s} x_{i s^{\prime}}\right)^{2}\right)^{1 / 2}=O(1)
$$


and therefore

$$
\left\{\frac{1}{N^{2}} \sum_{t=1}^{T} \sum_{i=1}^{N} v_{i t}^{2}\left[\left(\frac{1}{T^{2}} \sum_{s=1}^{T} \sum_{s^{\prime}, s \neq s^{\prime}}^{T}\left(x_{i s} x_{i s^{\prime}}\right)^{2}\right)^{1 / 2}\right]\right\}=O_{p}\left(T N^{-1}\right) .
$$

Further,

$$
E\left(\frac{1}{T} \sum_{s=1}^{T}\left\|\hat{\mathbf{f}}_{s}-\mathbf{f}_{s}\right\|_{F}^{2}\right)=O\left[\min (N, T)^{-1}\right],
$$

and overall we have $C_{4}=O_{p}\left(\frac{T}{N \min (N, T)}\right)$. Finally,

$$
\begin{aligned}
& \frac{1}{N^{2}} \sum_{t=1}^{T} \sum_{i=1}^{N} v_{i t}^{2}\left[\frac{1}{T^{2}} \sum_{s=1}^{T} \sum_{s^{\prime}=1}^{T} x_{i s^{\prime}} v_{i s} \mathbf{f}_{s}^{\prime}\left(\hat{\mathbf{f}}_{s^{\prime}}-\mathbf{f}_{s^{\prime}}\right)\right] \\
& =\left[\frac{1}{N^{2}} \sum_{t=1}^{T} \sum_{i=1}^{N} v_{i t}^{2}\left(\frac{1}{T} \sum_{s=1}^{T} v_{i s} \mathbf{f}_{s}^{\prime}\right)\right]\left[\frac{1}{T} \sum_{s=1}^{T} x_{i s^{\prime}}\left(\hat{\mathbf{f}}_{s^{\prime}}-\mathbf{f}_{s^{\prime}}\right)\right] .
\end{aligned}
$$

But using Lemma A.1 of Bai (2003) and $\sup _{i, t} E\left(x_{i t}^{2}\right)<\infty$,

$$
\left\|\frac{1}{T} \sum_{s=1}^{T} x_{i s^{\prime}}\left(\hat{\mathbf{f}}_{s^{\prime}}-\mathbf{f}_{s^{\prime}}\right)\right\|_{F}=O_{p}\left[\min (N, T)^{-1}\right]
$$

and

$$
\frac{1}{N^{2}} \sum_{t=1}^{T} \sum_{i=1}^{N} v_{i t}^{2}\left(\frac{1}{T} \sum_{s=1}^{T} v_{i s} \mathbf{f}_{s}^{\prime}\right)=O_{p}\left(T N^{-1}\right)
$$

So, we have

$$
\frac{1}{N^{2}} \sum_{t=1}^{T} \sum_{i=1}^{N} v_{i t}^{2}\left[\frac{1}{T^{2}} \sum_{s=1}^{T} \sum_{s^{\prime}=1}^{T} x_{i s^{\prime}} v_{i s} \mathbf{f}_{s}^{\prime}\left(\hat{\mathbf{f}}_{s^{\prime}}-\mathbf{f}_{s^{\prime}}\right)\right]=O_{p}\left[T N^{-1} \min (N, T)^{-1}\right],
$$

and hence

$$
\left\|\frac{\mathbf{V}\left(\hat{\mathbf{A}}-\mathbf{A}_{0}\right)}{N}\right\|_{F}=O_{p}\left(N^{-1 / 2}\right)+O_{p}\left(\frac{\sqrt{T}}{\sqrt{N \min (N, T)}}\right)=O_{p}\left(\frac{\sqrt{T}}{\sqrt{N \min (N, T)}}\right) .
$$

Lemma 4 Under Assumptions 1-4,

$$
\frac{\left\|\hat{\mathbf{F}}-\mathbf{F}_{0}\right\|_{F}^{2}}{T}=O_{p}\left(\frac{1}{\min (N, T)}\right) .
$$

Proof.

Since

$$
\frac{1}{T}\left\|\hat{\mathbf{F}}-\mathbf{F}_{0}\right\|_{F}^{2}=\frac{1}{T} \sum_{t=1}^{T}\left\|\hat{\mathbf{f}}_{t}-\mathbf{f}_{t}\right\|_{F}^{2},
$$

then the required result follows immediately from Theorem 1 of Bai and $\mathrm{Ng}$ (2002). 
Lemma 5 Under Assumptions 1-4,

$$
\left\|\frac{\mathbf{V}^{\prime}\left(\hat{\mathbf{F}}-\mathbf{F}_{0}\right)}{T}\right\|_{F}=O_{p}\left(\frac{\sqrt{N}}{T}\right)+O_{p}\left(\frac{1}{\sqrt{T}}\right)+O_{p}\left(\frac{N^{1 / 4}}{T^{3 / 4}}\right) .
$$

Proof. We have that

$$
\left\|\frac{\mathbf{V}^{\prime}\left(\hat{\mathbf{F}}-\mathbf{F}_{0}\right)}{T}\right\|_{F}^{2}=\sum_{i=1}^{N} \sum_{j=1}^{m}\left[\frac{1}{T} \sum_{t=1}^{T} v_{i t}\left(\hat{f}_{j t}-f_{j t}\right)\right]^{2} \leq \max _{j} \sum_{i=1}^{N}\left[\frac{1}{T} \sum_{t=1}^{T} v_{i t}\left(\hat{f}_{j t}-f_{j t}\right)\right]^{2} .
$$

But,

$$
\begin{aligned}
\sum_{i=1}^{N}\left[\frac{1}{T} \sum_{t=1}^{T} v_{i t}\left(\hat{f}_{j t}-f_{j t}\right)\right]^{2} & =\frac{1}{T^{2}} \sum_{i=1}^{N} \sum_{t=1}^{T} v_{i t}^{2}\left(\hat{f}_{j t}-f_{j t}\right)^{2}+ \\
\frac{2}{T^{2}} \sum_{i=1}^{N} \sum_{t=1}^{T} \sum_{s=1}^{T} v_{i t} v_{i s}\left(\hat{f}_{j t}-f_{j t}\right)\left(\hat{f}_{j s}-f_{j s}\right) & =A_{1}+A_{2} .
\end{aligned}
$$

By equation (58) we can write

$$
\hat{f}_{j t}-f_{j t}=\frac{1}{T} \sum_{l=1}^{T} \hat{f}_{j l} \gamma_{l t}+\frac{1}{T} \sum_{l=1}^{T} \hat{f}_{j l} \zeta_{l t}+\frac{1}{T} \sum_{l=1}^{T} \hat{f}_{j l} \varkappa_{l t}+\frac{1}{T} \sum_{l=1}^{T} \hat{f}_{j l} \xi_{l t}
$$

where $\zeta_{l t}=N^{-1} \mathbf{v}_{l}^{\prime} \mathbf{v}_{t}-\gamma_{l t}, \varkappa_{l t}=N^{-1} \mathbf{f}_{l}^{\prime} \mathbf{A}_{0}^{\prime} \mathbf{v}_{t}$, and $\xi_{l t}=\varkappa_{t l}$. We have

$$
\begin{aligned}
\frac{1}{T^{2}} \sum_{i=1}^{N} \sum_{t=1}^{T} v_{i t}^{2}\left(\hat{f}_{j t}-f_{j t}\right)^{2} & \leq \frac{4}{T^{2}} \sum_{i=1}^{N} \sum_{t=1}^{T} v_{i t}^{2}\left(\frac{1}{T} \sum_{l=1}^{T} \hat{f}_{j l} \gamma_{l t}\right)^{2}+ \\
& \frac{4}{T^{2}} \sum_{i=1}^{N} \sum_{t=1}^{T} v_{i t}^{2}\left(\frac{1}{T} \sum_{l=1}^{T} \hat{f}_{j l} \zeta_{l t}\right)^{2}+ \\
& \frac{4}{T^{2}} \sum_{i=1}^{N} \sum_{t=1}^{T} v_{i t}^{2}\left(\frac{1}{T} \sum_{l=1}^{T} \hat{f}_{j l} \varkappa_{l t}\right)^{2}+ \\
& \frac{4}{T^{2}} \sum_{i=1}^{N} \sum_{t=1}^{T} v_{i t}^{2}\left(\frac{1}{T} \sum_{l=1}^{T} \hat{f}_{j l} \xi_{l t}\right)^{2} \\
& =A_{11}+A_{12}+A_{13}+A_{14} .
\end{aligned}
$$

Now,

$$
A_{11}=\frac{1}{T^{4}} \sum_{i=1}^{N} \sum_{t=1}^{T} v_{i t}^{2}\left(\sum_{l=1}^{T} \hat{f}_{j l} \gamma_{l t}\right)^{2} \leq\left(\frac{1}{T} \sum_{l=1}^{T} \hat{f}_{j l}^{2}\right) \frac{1}{T^{3}} \sum_{i=1}^{N} \sum_{t=1}^{T} v_{i t}^{2}\left(\sum_{l=1}^{T} \gamma_{l t}^{2}\right) .
$$

But $T^{-1} \sum_{l=1}^{T} \hat{f}_{j l}^{2}=O_{p}(1)$, and $\sum_{l=1}^{T} \gamma_{l t}^{2}<C$. Hence

$$
\sum_{i=1}^{N} \sum_{t=1}^{T} v_{i t}^{2}\left(\sum_{l=1}^{T} \gamma_{l t}^{2}\right) \leq C \sum_{i=1}^{N} \sum_{t=1}^{T} v_{i t}^{2}=O_{p}(T N)
$$


So

$$
A_{11}=\frac{1}{T^{4}} \sum_{i=1}^{N} \sum_{t=1}^{T} v_{i t}^{2}\left(\sum_{l=1}^{T} \hat{f}_{j l} \gamma_{l t}\right)^{2}=O_{p}\left(N T^{-2}\right) .
$$

Next

$$
\begin{aligned}
A_{12} & =\frac{1}{T^{2}} \sum_{i=1}^{N} \sum_{t=1}^{T} v_{i t}^{2}\left(\frac{1}{T} \sum_{l=1}^{T} \hat{f}_{j l} \zeta_{l t}\right)^{2}=\frac{1}{T^{4}} \sum_{i=1}^{N} \sum_{t=1}^{T} v_{i t}^{2}\left(\sum_{l=1}^{T} \hat{f}_{j l} \zeta_{l t}\right)^{2} \\
& =\frac{1}{T^{4}}\left(\sum_{l=1}^{T} \sum_{u=1}^{T} \hat{f}_{j l} \hat{f}_{j u} \sum_{t=1}^{T} \zeta_{l t} \zeta_{u t}\left(\sum_{i=1}^{N} v_{i t}^{2}\right) \leq\right. \\
& \frac{N}{T^{2}}\left[\frac{1}{T^{2}} \sum_{l=1}^{T} \sum_{u=1}^{T}\left(\hat{f}_{j l} \hat{f}_{j u}\right)^{2}\right]^{1 / 2}\left\{\frac{1}{T^{2}} \sum_{l=1}^{T} \sum_{u=1}^{T}\left[\sum_{t=1}^{T} \zeta_{l t} \zeta_{u t}\left(\frac{1}{N} \sum_{i=1}^{N} v_{i t}^{2}\right)\right]^{2}\right\}^{1 / 2} \\
& \leq \frac{N}{T^{2}}\left(\frac{1}{T} \sum_{l=1}^{T} \hat{f}_{j l}^{2}\right)\left\{\frac{1}{T^{2}} \sum_{l=1}^{T} \sum_{u=1}^{T}\left[\sum_{t=1}^{T} \zeta_{l t} \zeta_{u t}\left(\frac{1}{N} \sum_{i=1}^{N} v_{i t}^{2}\right)\right]^{2}\right\}^{1 / 2} .
\end{aligned}
$$

But

$$
E\left[\left(\sum_{t=1}^{T} \zeta_{l t} \zeta_{u t}\left(\frac{1}{N} \sum_{i=1}^{N} v_{i t}^{2}\right)\right)^{2}\right] \leq T^{2} N^{-2}
$$

So

$$
A_{12}=\frac{N}{T^{2}} \cdot O_{p}(1) \cdot \sqrt{T^{2} N^{-2}}=O_{p}\left(T^{-1}\right) .
$$

Next,

$$
\begin{aligned}
A_{13} & =\frac{4}{T^{2}} \sum_{i=1}^{N} \sum_{t=1}^{T} v_{i t}^{2}\left(\frac{1}{T} \sum_{l=1}^{T} \hat{f}_{j l} \varkappa_{l t}\right)^{2}=\frac{4}{N^{2} T^{2}} \sum_{i=1}^{N} \sum_{t=1}^{T} v_{i t}^{2} \frac{1}{T^{2}}\left(\sum_{l=1}^{T} \hat{f}_{j l} \mathbf{f}_{l}^{\prime} \mathbf{A}_{0}^{\prime} \mathbf{v}_{t}\right)^{2} \leq \\
& \left(\frac{1}{T} \sum_{l=1}^{T} \hat{f}_{j l}^{2}\right)\left(\frac{1}{T} \sum_{l=1}^{T}\left\|\mathbf{f}_{l}\right\|^{2}\right) \frac{4}{N T^{2}} \sum_{t=1}^{T}\left(\frac{1}{N} \sum_{i=1}^{N} v_{i t}^{2}\right)\left\|\mathbf{A}_{0}^{\prime} \mathbf{v}_{t}\right\|^{2} \\
& =\left(\frac{1}{T} \sum_{l=1}^{T} \hat{f}_{j l}^{2}\right)\left(\frac{1}{T} \sum_{l=1}^{T}\left\|\mathbf{f}_{l}\right\|^{2}\right) \frac{4}{T^{2}} \sum_{t=1}^{T}\left(\frac{1}{N} \sum_{i=1}^{N} v_{i t}^{2}\right)\left\|\frac{\mathbf{A}_{0}^{\prime} \mathbf{v}_{t}}{\sqrt{N}}\right\|^{2}=O_{p}\left(T^{-1}\right) .
\end{aligned}
$$

Similarly for $A_{14}$. So, overall

$$
A_{1}=O_{p}\left(N T^{-2}\right)+O_{p}\left(T^{-1}\right)=O_{p}\left(\frac{N}{T \min (N, T)}\right) .
$$

Next we consider $A_{2}$, and note that 


$$
\begin{aligned}
\left(\hat{f}_{j t}-f_{j t}\right)\left(\hat{f}_{j s}-f_{j s}\right) & =\left(\frac{1}{T} \sum_{l=1}^{T} \hat{f}_{j l} \gamma_{l t}+\frac{1}{T} \sum_{l=1}^{T} \hat{f}_{j l} \zeta_{l t}+\frac{1}{T} \sum_{l=1}^{T} \hat{f}_{j l} \varkappa_{l t}+\frac{1}{T} \sum_{l=1}^{T} \hat{f}_{j l} \xi_{l t}\right) \\
\times & \left(\frac{1}{T} \sum_{l=1}^{T} \hat{f}_{j l} \gamma_{l s}+\frac{1}{T} \sum_{l=1}^{T} \hat{f}_{j l} \zeta_{l s}+\frac{1}{T} \sum_{l=1}^{T} \hat{f}_{j l} \varkappa_{l s}+\frac{1}{T} \sum_{l=1}^{T} \hat{f}_{j l} \xi_{l s}\right) \\
& =\frac{1}{T^{2}} \sum_{l=1}^{T} \sum_{u=1}^{T} \hat{f}_{j l} \hat{f}_{j u} \gamma_{l t} \gamma_{u s}+\frac{2}{T^{2}} \sum_{l=1}^{T} \sum_{u=1}^{T} \hat{f}_{j l} \hat{f}_{j u} \gamma_{l t} \zeta_{u s}+ \\
& \frac{2}{T^{2}} \sum_{l=1}^{T} \sum_{u=1}^{T} \hat{f}_{j l} \hat{f}_{j u} \gamma_{l t} \varkappa_{u s}+\frac{2}{T^{2}} \sum_{l=1}^{T} \sum_{u=1}^{T} \hat{f}_{j l} \hat{f}_{j u} \gamma_{l t} \xi_{u s}+ \\
& \frac{1}{T^{2}} \sum_{l=1}^{T} \sum_{u=1}^{T} \hat{f}_{j l} \hat{f}_{j u} \zeta_{l t} \zeta_{u s}+\frac{2}{T^{2}} \sum_{l=1}^{T} \sum_{u=1}^{T} \hat{f}_{j l} \hat{f}_{j u} \zeta_{l t} \varkappa_{u s}+\frac{2}{T^{2}} \sum_{l=1}^{T} \sum_{u=1}^{T} \hat{f}_{j l} \hat{f}_{j u} \zeta_{l t} \xi_{u s}+ \\
& \frac{1}{T^{2}} \sum_{l=1}^{T} \sum_{u=1}^{T} \hat{f}_{j l} \hat{f}_{j u} \varkappa_{l t} \varkappa_{u s}+\frac{2}{T^{2}} \sum_{l=1}^{T} \sum_{u=1}^{T} \hat{f}_{j l} \hat{f}_{j u} \varkappa_{l t} \xi_{u s}+\frac{1}{T^{2}} \sum_{l=1}^{T} \sum_{u=1}^{T} \hat{f}_{j l} \hat{f}_{j u} \xi_{l t} \xi_{u s} .
\end{aligned}
$$

Therefore

$$
A_{2}=\frac{2}{T^{2}} \sum_{i=1}^{N} \sum_{t=1}^{T} \sum_{s=1}^{T} v_{i t} v_{i s}\left(\hat{f}_{j t}-f_{j t}\right)\left(\hat{f}_{j s}-f_{j s}\right)=\sum_{i=1}^{10} A_{2 i} .
$$

Denoting equality in order of probability by $A \sim B$, we proceed term by term noting that $A_{23} \sim A_{24}, A_{26} \sim A_{27}$ and $A_{28} \sim A_{210}$. So the terms to consider are $A_{21}, A_{22}, A_{23}, A_{25}, A_{26}$, $A_{28}$ and $A_{29}$. Starting with $A_{21}$ we have

$$
\begin{aligned}
A_{21} & =\frac{2}{T^{4}} \sum_{i=1}^{N} \sum_{t=1}^{T} \sum_{s=1}^{T} v_{i t} v_{i s}\left(\sum_{l=1}^{T} \sum_{u=1}^{T} \hat{f}_{j l} \hat{f}_{j u} \gamma_{l t} \gamma_{u s}\right) \\
& =\frac{2}{T^{4}} \sum_{i=1}^{N} \sum_{l=1}^{T} \sum_{u=1}^{T} \hat{f}_{j l} \hat{f}_{j u}\left(\sum_{t=1}^{T} \sum_{s=1}^{T} v_{i t} v_{i s} \gamma_{l t} \gamma_{u s}\right) \\
& \left.\leq \frac{2}{T^{2}} \sum_{i=1}^{N}\left\{\left[\frac{1}{T^{2}} \sum_{l=1}^{T} \sum_{u=1}^{T}\left(\hat{f}_{j l} \hat{f}_{j u}\right)^{2}\right)^{1 / 2} \sum_{l=1}^{T} \sum_{u=1}^{T}\left(\sum_{t=1}^{T} \sum_{s=1}^{T} v_{i t} v_{i s} \gamma_{l t} \gamma_{u s}\right)^{2}\right]^{1 / 2}\right\} \\
= & \left\{\frac{2}{T^{2}} \sum_{i=1}^{N}\left[\frac{1}{T^{2}} \sum_{l=1}^{T} \sum_{u=1}^{T}\left(\sum_{t=1}^{T} \sum_{s=1}^{T} v_{i t} v_{i s} \gamma_{l t} \gamma_{u s}\right)^{1 / 2}\right\}\left(\frac{1}{T} \sum_{u=1}^{T} \hat{f}_{j u}^{2}\right) .\right.
\end{aligned}
$$

But, due to summability of $\gamma_{l t}$

$$
E\left[\left(\sum_{t=1}^{T} \sum_{s=1}^{T} v_{i t} v_{i s} \gamma_{l t} \gamma_{u s}\right)^{2}\right] \leq T C
$$


Noting further that, again due to summability of $\gamma_{l t}$, the double sum over $l$ and $u$ will only have terms bounded away from zero if $l$ and $u$ are close we obtain

$$
E\left[\frac{1}{T^{2}} \sum_{l=1}^{T} \sum_{u=1}^{T}\left(\sum_{t=1}^{T} \sum_{s=1}^{T} v_{i t} v_{i s} \gamma_{l t} \gamma_{u s}\right)^{2}\right]=O(1)
$$

and hence $A_{21}=O_{p}\left(N T^{-2}\right)$. Consider now

$$
\begin{aligned}
A_{22} & =\frac{2}{T^{4}} \sum_{i=1}^{N} \sum_{t=1}^{T} \sum_{s=1}^{T} v_{i t} v_{i s}\left(\sum_{l=1}^{T} \sum_{u=1}^{T} \hat{f}_{j l} \hat{f}_{j u} \gamma_{l t} \zeta_{u s}\right) \\
= & \frac{2}{T^{4}} \sum_{i=1}^{N} \sum_{l=1}^{T} \sum_{u=1}^{T} \hat{f}_{j l} \hat{f}_{j u}\left(\sum_{t=1}^{T} \sum_{s=1}^{T} v_{i t} v_{i s} \gamma_{l t} \zeta_{u s}\right) \\
& \left.\left.\leq \frac{2}{T^{3 / 2}} \sum_{i=1}^{N}\left[\left[\frac{1}{T^{2}} \sum_{l=1}^{T} \sum_{u=1}^{T}\left(\sum_{t=1}^{T} \sum_{s=1}^{T} v_{i t} v_{i s} \gamma_{l t} \zeta_{u s}\right)^{2}\right]^{1 / 2} \hat{f}_{j l} \hat{f}_{j u}\right)^{2}\right)^{1 / 2}\right] \\
& \leq\left[\frac{2}{T^{3 / 2}} \sum_{i=1}^{N}\left[\left[\frac{1}{T^{3}} \sum_{l=1}^{T} \sum_{u=1}^{T}\left(\sum_{t=1}^{T} \sum_{s=1}^{T} v_{i t} v_{i s} \gamma_{l t} \zeta_{u s}\right)^{2 / 2}\right]\right] \times\left(\frac{1}{T} \sum_{u=1}^{T} \hat{f}_{j u}^{2}\right) .\right.
\end{aligned}
$$

But

$$
E\left[\left(\sum_{t=1}^{T} \sum_{s=1}^{T} v_{i t} v_{i s} \gamma_{l t} \zeta_{u s}\right)^{2}\right] \leq T^{2} N^{-1}
$$

Further, due to summability of $\gamma_{l t}$ the double sum over $l$ and $t$ will only have terms bounded away from zero if $l$ and $t$ are close so

$$
E\left[\frac{1}{T^{3}} \sum_{l=1}^{T} \sum_{u=1}^{T}\left(\sum_{t=1}^{T} \sum_{s=1}^{T} v_{i t} v_{i s} \gamma_{l t} \zeta_{u s}\right)^{2}\right]=O\left(N^{-1}\right)
$$

and as a result $A_{22}=O_{p}\left(N^{1 / 2} T^{-3 / 2}\right)$. Next, and similarly to the previous terms

$$
\begin{aligned}
A_{23} & =\frac{2}{T^{4}} \sum_{i=1}^{N} \sum_{t=1}^{T} \sum_{s=1}^{T} v_{i t} v_{i s}\left(\sum_{l=1}^{T} \sum_{u=1}^{T} \hat{f}_{j l} \hat{f}_{j u} \gamma_{l t} \varkappa_{u s}\right) \leq \\
& \leq\left(\frac{2}{T^{3 / 2}} \sum_{i=1}^{N}\left\{\left[\frac{1}{T^{3}} \sum_{l=1}^{T} \sum_{u=1}^{T}\left(\sum_{t=1}^{T} \sum_{s=1}^{T} v_{i t} v_{i s} \gamma_{l t} \varkappa_{u s}\right)^{2}\right]^{1 / 2}\right\}\right)\left(\frac{1}{T} \sum_{u=1}^{T} \hat{f}_{j u}^{2}\right),
\end{aligned}
$$


which again, by a manipulation similar to that used for (65), yields $A_{23}=O_{p}\left(N^{1 / 2} T^{-3 / 2}\right)$. Next,

$$
\begin{aligned}
A_{25} & =\frac{2}{T^{4}} \sum_{i=1}^{N} \sum_{t=1}^{T} \sum_{s=1}^{T} v_{i t} v_{i s}\left(\sum_{l=1}^{T} \sum_{u=1}^{T} \hat{f}_{j l} \hat{f}_{j u} \zeta_{l t} \zeta_{u s}\right) \\
& =\frac{2}{T^{4}} \sum_{i=1}^{N}\left[\sum_{l=1}^{T} \sum_{u=1}^{T} \hat{f}_{j l} \hat{f}_{j u}\left(\sum_{t=1}^{T} \sum_{s=1}^{T} \zeta_{l t} \zeta_{u s} v_{i t} v_{i s}\right)\right] \\
& \leq \frac{2}{T} \sum_{i=1}^{N}\left\{\left[\frac{1}{T^{2}} \sum_{l=1}^{T} \sum_{u=1}^{T}\left(\hat{f}_{j l} \hat{f}_{j u}\right)^{2}\right)^{1 / 2}\right. \\
& \left.\left.\left.\leq \frac{2}{T} \sum_{i=1}^{N}\left\{\left(\frac{1}{T} \sum_{u=1}^{T} \hat{f}_{j u}^{2}\right)\left[\frac{1}{T^{4}} \sum_{l=1}^{T} \sum_{u=1}^{T}\left(\sum_{t=1}^{T} \sum_{s=1}^{T} \zeta_{l t} \zeta_{u s} v_{i t} v_{i s} v_{i s}\right)^{T}\right]^{T}\right]^{1 / 2} \zeta_{l t} \zeta_{u s} v_{i t} v_{i s}\right)^{2}\right]^{1 / 2}\right\} .
\end{aligned}
$$

But, by absolute summability of the autocovariance of $v_{i t}$,

$$
E\left[\left(\sum_{t=1}^{T} \sum_{s=1}^{T} \zeta_{l t} \zeta_{u s} v_{i t} v_{i s}\right)^{2}\right] \leq C T^{2} E\left(\zeta_{l t}^{4}\right) \leq C T^{4} N^{-2}
$$

So

$$
\begin{gathered}
{\left[\frac{1}{T^{4}} \sum_{l=1}^{T} \sum_{u=1}^{T}\left(\sum_{t=1}^{T} \sum_{s=1}^{T} \zeta_{l t} \zeta_{u s} v_{i t} v_{i s}\right)^{2}\right]^{1 / 2}=O_{p}\left(N^{-1}\right),} \\
\frac{2}{T} \sum_{i=1}^{N}\left\{\left(\frac{1}{T} \sum_{u=1}^{T} \hat{f}_{j u}^{2}\right)\left[\frac{1}{T^{4}} \sum_{l=1}^{T} \sum_{u=1}^{T}\left(\sum_{t=1}^{T} \sum_{s=1}^{T} \zeta_{l t} \zeta_{u s} v_{i t} v_{i s}\right)^{2}\right]^{1 / 2}\right\}=O_{p}\left(T^{-1}\right),
\end{gathered}
$$

and $A_{25}=O_{p}\left(T^{-1}\right)$. Next

$$
\begin{aligned}
A_{26} & =\frac{2}{T^{4}} \sum_{i=1}^{N} \sum_{t=1}^{T} \sum_{s=1}^{T} v_{i t} v_{i s}\left(\sum_{l=1}^{T} \sum_{u=1}^{T} \hat{f}_{j l} \hat{f}_{j u} \zeta_{l t} \varkappa_{u s}\right) \leq \\
& \leq\left(\frac{2}{T} \sum_{i=1}^{N}\left\{\left[\frac{1}{T^{4}} \sum_{l=1}^{T} \sum_{u=1}^{T}\left(\sum_{t=1}^{T} \sum_{s=1}^{T} v_{i t} v_{i s} \zeta_{l t} \varkappa_{u s}\right)^{2}\right]^{1 / 2}\right\}\right)\left(\frac{1}{T} \sum_{u=1}^{T} \hat{f}_{j u}^{2}\right) .
\end{aligned}
$$

But

$$
E\left[\left(\sum_{t=1}^{T} \sum_{s=1}^{T} v_{i t} v_{i s} \zeta_{l t} \varkappa_{u s}\right)^{2}\right] \leq T^{2} N^{-2}
$$

and

$$
E\left[\frac{1}{T^{3}} \sum_{l=1}^{T} \sum_{u=1}^{T}\left(\sum_{t=1}^{T} \sum_{s=1}^{T} v_{i t} v_{i s} \zeta_{l t} \varkappa_{u s}\right)^{2}\right]=O\left(N^{-2}\right) .
$$


So $A_{26}=O_{p}\left(T^{-1}\right)$. Similarly, we obtain $A_{28}=O_{p}\left(T^{-1}\right)$ and $A_{29}=O_{p}\left(T^{-1}\right)$. Collecting the terms, we have

$$
A_{2}=O_{p}\left(N T^{-2}\right)+O_{p}\left(N^{1 / 2} T^{-3 / 2}\right)+O_{p}\left(T^{-1}\right)
$$

Thus

$$
\left\|\frac{\mathbf{V}^{\prime}\left(\hat{\mathbf{F}}-\mathbf{F}_{0}\right)}{T}\right\|_{F}^{2}=O_{p}\left(N T^{-2}\right)+O_{p}\left(T^{-1}\right)+O_{p}\left(N^{1 / 2} T^{-3 / 2}\right),
$$

and hence

$$
\left\|\frac{\mathbf{V}^{\prime}\left(\hat{\mathbf{F}}-\mathbf{F}_{0}\right)}{T}\right\|_{F}=O_{p}\left(N^{1 / 2} T^{-1}\right)+O_{p}\left(T^{-1 / 2}\right)+O_{p}\left(N^{1 / 4} T^{-3 / 4}\right) .
$$

Lemma 6 Under Assumptions 1-4,

$$
\frac{\left\|\hat{\mathbf{A}}-\mathbf{A}_{0}\right\|_{F}^{2}}{N}=O_{p}\left(\frac{1}{\min (N, T)}\right)
$$

and

$$
\left\|\mathbf{A}_{0}^{\prime}\left(\hat{\mathbf{A}}-\mathbf{A}_{0}\right)\right\|_{F}=O_{p}\left(\frac{N}{\sqrt{\min (N, T)}}\right) .
$$

Proof. We have by the proof of Theorem 2 of Bai (2003, expression above (B.2)) that

$$
\hat{\mathbf{a}}_{i}-\mathbf{a}_{i}=\frac{1}{T} \sum_{t=1}^{T} \mathbf{f}_{t} v_{i t}+\frac{1}{T} \sum_{t=1}^{T} x_{i t}\left(\hat{\mathbf{f}}_{t}-\mathbf{f}_{t}\right) .
$$


This result can be used to obtain

$$
\begin{aligned}
\frac{1}{N}\left\|\left(\hat{\mathbf{A}}-\mathbf{A}_{0}\right)\right\|_{F}^{2} & =\frac{1}{N} \sum_{i=1}^{N}\left(\hat{\mathbf{a}}_{i}-\mathbf{a}_{i}\right)^{\prime}\left(\hat{\mathbf{a}}_{i}-\mathbf{a}_{i}\right) \\
& \leq \frac{1}{N} \sum_{i=1}^{N}\left(\frac{1}{T^{2}} \sum_{s=1}^{T} \mathbf{f}_{s}^{\prime} \mathbf{f}_{s} v_{i s}^{2}+\frac{1}{T^{2}} \sum_{s=1}^{T} \sum_{s^{\prime}, s \neq s^{\prime}}^{T} \mathbf{f}_{s}^{\prime} \mathbf{f}_{s^{\prime}} v_{i s} v_{i s^{\prime}}\right)+ \\
& \frac{1}{N} \sum_{i=1}^{N}\left(\frac{1}{T^{2}} \sum_{s=1}^{T} \sum_{s^{\prime}, s \neq s^{\prime}}^{T}\left|x_{i s} x_{i s^{\prime}}\right|\left\|\hat{\mathbf{f}}_{s}-\mathbf{f}_{s}\right\|_{F}\left\|\hat{\mathbf{f}}_{s^{\prime}}-\mathbf{f}_{s^{\prime}}\right\|_{F}\right)+ \\
& \frac{2}{N} \sum_{i=1}^{N}\left(\frac{1}{T^{2}} \sum_{s=1}^{T} \sum_{s^{\prime}=1}^{T} x_{i s^{\prime}} v_{i s} \mathbf{f}_{s}^{\prime}\left(\hat{\mathbf{f}}_{s^{\prime}}-\mathbf{f}_{s^{\prime}}\right)\right)= \\
& \frac{1}{N} \sum_{i=1}^{N}\left(\frac{1}{T^{2}} \sum_{s=1}^{T} \mathbf{f}_{s}^{\prime} \mathbf{f}_{s} v_{i s}^{2}\right)+ \\
& \frac{1}{N} \sum_{i=1}^{N}\left(\frac{1}{T^{2}} \sum_{s=1}^{T} \sum_{s^{\prime}, s \neq s^{\prime}}^{T} \mathbf{f}_{s}^{\prime} \mathbf{f}_{s^{\prime}} v_{i s} v_{i s^{\prime}}\right)+ \\
& \frac{1}{N} \sum_{i=1}^{N}\left(\frac{1}{T^{2}} \sum_{s=1}^{T} x_{i s}^{2}\left\|\hat{\mathbf{f}}_{s}-\mathbf{f}_{s}\right\|_{F}^{2}\right)+ \\
& \frac{1}{N} \sum_{i=1}^{N}\left(\frac{1}{T^{2}} \sum_{s=1}^{T} \sum_{s^{\prime}, s \neq s^{\prime}}^{T}\left|x_{i s} x_{i s^{\prime}}\right|\left\|\hat{\mathbf{f}}_{s}-\mathbf{f}_{s}\right\|_{F}\left\|\hat{\mathbf{f}}_{s^{\prime}}-\mathbf{f}_{s^{\prime}}\right\|_{F}\right)+ \\
& \frac{2}{N} \sum_{i=1}^{N}\left(\frac{1}{T^{2}} \sum_{s=1}^{T} \sum_{s^{\prime}=1}^{T} x_{i s^{\prime}} v_{i s} \mathbf{f}_{s}^{\prime}\left(\hat{\mathbf{f}}_{s^{\prime}}-\mathbf{f}_{s^{\prime}}\right)\right) \\
& =\sum_{i=1}^{5} C_{i} . \\
&
\end{aligned}
$$

We have

$$
C_{1}=\frac{1}{N} \sum_{i=1}^{N}\left(\frac{1}{T^{2}} \sum_{s=1}^{T} \mathbf{f}_{s}^{\prime} \mathbf{f}_{s} v_{i s}^{2}\right)=O_{p}\left(T^{-1}\right) .
$$

Also

$$
\begin{aligned}
C_{2} & =\frac{1}{N} \sum_{i=1}^{N}\left(\frac{1}{T^{2}} \sum_{s=1}^{T} \sum_{s^{\prime}, s \neq s^{\prime}}^{T} \mathbf{f}_{s}^{\prime} \mathbf{f}_{s^{\prime}} v_{i s} v_{i s^{\prime}}\right) \\
& =\frac{1}{N} \frac{1}{T} \sum_{i=1}^{N}\left[\frac{1}{\sqrt{T}} \sum_{s=1}^{T} v_{i s} \mathbf{f}_{s}^{\prime}\left(\frac{1}{\sqrt{T}} \sum_{s^{\prime}, s \neq s^{\prime}}^{T} \mathbf{f}_{s^{\prime}} v_{i s^{\prime}}\right)\right]=O_{p}\left(T^{-1}\right),
\end{aligned}
$$


and

$$
\begin{aligned}
C_{3} & =\frac{1}{N} \sum_{i=1}^{N}\left(\frac{1}{T^{2}} \sum_{s=1}^{T} x_{i s}^{2}\left\|\hat{\mathbf{f}}_{s}-\mathbf{f}_{s}\right\|_{F}^{2}\right) \\
& =\frac{1}{\min (N, T) N T} \sum_{i=1}^{N}\left(\frac{\min (N, T)}{T} \sum_{s=1}^{T} x_{i s}^{2}\left\|\hat{\mathbf{f}}_{s}-\mathbf{f}_{s}\right\|_{F}^{2}\right)=O_{p}\left(T^{-1} \min (N, T)^{-1}\right),
\end{aligned}
$$

noting that by Lemma A.1 of Bai (2003) and $\sup _{i} \sup _{t} E\left(x_{i t}^{2}\right)<\infty$,

$$
\sup _{i} E\left(\frac{\min (N, T)}{T} \sum_{s=1}^{T} x_{i s}^{2}\left\|\hat{\mathbf{f}}_{s}-\mathbf{f}_{s}\right\|_{F}^{2}\right)^{2}=O(1) .
$$

Further,

$$
\begin{aligned}
& C_{4}=\frac{1}{N} \sum_{i=1}^{N}\left(\frac{1}{T^{2}} \sum_{s=1}^{T} \sum_{s^{\prime}, s \neq s^{\prime}}^{T}\left|x_{i s} x_{i s^{\prime}}\right|\left\|\hat{\mathbf{f}}_{s}-\mathbf{f}_{s}\right\|_{F}\left\|\hat{\mathbf{f}}_{s^{\prime}}-\mathbf{f}_{s^{\prime}}\right\|_{F}\right) \\
& \leq \frac{1}{N} \sum_{i=1}^{N}\left[\begin{array}{c}
\left(\frac{1}{T^{2}} \sum_{s=1}^{T} \sum_{s^{\prime}, s \neq s^{\prime}}^{T}\left(x_{i s} x_{i s^{\prime}}\right)^{2}\right)^{1 / 2} \\
\left(\frac{1}{T^{2}} \sum_{s=1}^{T} \sum_{s^{\prime}, s \neq s^{\prime}}^{T}\left\|\hat{\mathbf{f}}_{s}-\mathbf{f}_{s}\right\|_{F}^{2}\left\|\hat{\mathbf{f}}_{s^{\prime}}-\mathbf{f}_{s^{\prime}}\right\|_{F}^{2}\right)^{1 / 2}
\end{array}\right] \\
& \leq \frac{1}{N} \sum_{i=1}^{N}\left[\begin{array}{c}
\left(\frac{1}{T^{2}} \sum_{s=1}^{T} \sum_{s^{\prime}, s \neq s^{\prime}}^{T}\left(x_{i s} x_{i s^{\prime}}\right)^{2}\right)^{1 / 2} \\
\left\{\frac{1}{T} \sum_{s=1}^{T}\left[\left\|\hat{\mathbf{f}}_{s}-\mathbf{f}_{s}\right\|_{F}^{2}\left(\frac{1}{T} \sum_{s^{\prime}, s \neq s^{\prime}}^{T}\left\|\hat{\mathbf{f}}_{s}-\mathbf{f}_{s}\right\|_{F}^{2}\right)\right]\right\}^{1 / 2}
\end{array}\right] \\
& \leq\left(\frac{1}{T} \sum_{s=1}^{T}\left\|\hat{\mathbf{f}}_{s}-\mathbf{f}_{s}\right\|_{F}^{2}\right)\left\{\frac{1}{N} \sum_{i=1}^{N}\left[\left(\frac{1}{T^{2}} \sum_{s=1}^{T} \sum_{s^{\prime}, s \neq s^{\prime}}^{T}\left(x_{i s} x_{i s^{\prime}}\right)^{2}\right)^{1 / 2}\right]\right\} \text {. }
\end{aligned}
$$

But

$$
\sup _{i} E\left(\frac{1}{T^{2}} \sum_{s=1}^{T} \sum_{s^{\prime}, s \neq s^{\prime}}^{T}\left(x_{i s} x_{i s^{\prime}}\right)^{2}\right)^{1 / 2}=O(1)
$$

and so

$$
\frac{1}{N} \sum_{i=1}^{N}\left[\left(\frac{1}{T^{2}} \sum_{s=1}^{T} \sum_{s^{\prime}, s \neq s^{\prime}}^{T}\left(x_{i s} x_{i s^{\prime}}\right)^{2}\right)^{1 / 2}\right]=O_{p}(1)
$$

Further,

$$
E\left(\frac{1}{T} \sum_{s=1}^{T}\left\|\hat{\mathbf{f}}_{s}-\mathbf{f}_{s}\right\|_{F}^{2}\right)=O\left[\min (N, T)^{-1}\right]
$$

So, overall $C_{4}=O_{p}\left(\frac{1}{\min (N, T)}\right)$. Finally, noting by Lemma A.1 of Bai (2003) (or can be proven by first principles) that

$$
\sup _{i}\left\{E\left[\frac{1}{T^{2}} \sum_{s=1}^{T} \sum_{s^{\prime}=1}^{T} x_{i s^{\prime}} v_{i s} \mathbf{f}_{s}^{\prime}\left(\hat{\mathbf{f}}_{s^{\prime}}-\mathbf{f}_{s^{\prime}}\right)\right]^{2}\right\}^{1 / 2}=O_{p}\left[\min (N, T)^{-1}\right]
$$


we have

$$
C_{5}=\frac{1}{N} \sum_{i=1}^{N}\left[\frac{1}{T^{2}} \sum_{s=1}^{T} \sum_{s^{\prime}=1}^{T} x_{i s^{\prime}} v_{i s} \mathbf{f}_{s}^{\prime}\left(\hat{\mathbf{f}}_{s^{\prime}}-\mathbf{f}_{s^{\prime}}\right)\right]=O_{p}\left[\min (N, T)^{-1}\right]
$$

So overall

$$
\frac{1}{N}\left\|\hat{\mathbf{A}}-\mathbf{A}_{0}\right\|_{F}^{2}=O_{p}\left(\frac{1}{\min (N, T)}\right) .
$$

To prove (66), recall that by equation (63),

$$
\hat{\mathbf{a}}_{i}-\mathbf{a}_{i}=\frac{1}{T} \sum_{t=1}^{T} \mathbf{f}_{t} v_{i t}+\frac{1}{T} \sum_{t=1}^{T} x_{i t}\left(\hat{\mathbf{f}}_{t}-\mathbf{f}_{t}\right) .
$$

Define $\mathbf{B}=\mathbf{A}_{0}^{\prime} \mathbf{A}_{0}$. Note that every element of $\mathbf{B}$ is $O(N)$ and so every element of $N^{-1} \mathbf{B}$ is bounded. We have

$$
\begin{aligned}
& \left\|\mathbf{A}_{0}^{\prime}\left(\hat{\mathbf{A}}-\mathbf{A}_{0}\right)\right\|_{F}^{2}=\operatorname{Tr}\left[\left(\hat{\mathbf{A}}-\mathbf{A}_{0}\right) \mathbf{A}_{0}^{\prime} \mathbf{A}_{0}\left(\hat{\mathbf{A}}-\mathbf{A}_{0}\right)^{\prime}\right]=\sum_{i=1}^{N}\left(\hat{\mathbf{a}}_{i}-\mathbf{a}_{i}\right)^{\prime} \mathbf{B}\left(\hat{\mathbf{a}}_{i}-\mathbf{a}_{i}\right) \\
& \leq \sum_{i=1}^{N}\left(\frac{1}{T^{2}} \sum_{s=1}^{T} \mathbf{f}_{s}^{\prime} \mathbf{B} \mathbf{f}_{s} v_{i s}^{2}+\frac{1}{T^{2}} \sum_{s=1}^{T} \sum_{s^{\prime}, s \neq s^{\prime}}^{T} \mathbf{f}_{s}^{\prime} \mathbf{B f}_{s^{\prime}} v_{i s} v_{i s^{\prime}}\right)+ \\
& \|\mathbf{B}\|_{F}\left[\sum_{i=1}^{N}\left(\begin{array}{c}
\frac{1}{T^{2}} \sum_{s=1}^{T} x_{i s}^{2}\left\|\hat{\mathbf{f}}_{s}-\mathbf{f}_{s}\right\|_{F}^{2}+ \\
\frac{1}{T^{2}} \sum_{s=1}^{T} \sum_{s^{\prime}, s \neq s^{\prime}}^{T}\left|x_{i s} x_{i s^{\prime}}\right|\left\|\hat{\mathbf{f}}_{s}-\mathbf{f}_{s}\right\|_{F}\left\|\hat{\mathbf{f}}_{s^{\prime}}-\mathbf{f}_{s^{\prime}}\right\|_{F}
\end{array}\right)\right]+ \\
& \sum_{i=1}^{N}\left[\frac{1}{T^{2}} \sum_{s=1}^{T} \sum_{s^{\prime}=1}^{T} x_{i s^{\prime}} v_{i s} \mathbf{f}_{s}^{\prime} \mathbf{B}\left(\hat{\mathbf{f}}_{s^{\prime}}-\mathbf{f}_{s^{\prime}}\right)\right]= \\
& \sum_{i=1}^{N}\left(\frac{1}{T^{2}} \sum_{s=1}^{T} \mathbf{f}_{s}^{\prime} \mathbf{B} \mathbf{f}_{s} v_{i s}^{2}\right)+\sum_{i=1}^{N}\left(\frac{1}{T^{2}} \sum_{s=1}^{T} \sum_{s^{\prime}, s \neq s^{\prime}}^{T} \mathbf{f}_{s}^{\prime} \mathbf{B} \mathbf{f}_{s^{\prime}} v_{i s} v_{i s^{\prime}}\right)+ \\
& \|\mathbf{B}\|_{F}\left[\sum_{i=1}^{N}\left(\frac{1}{T^{2}} \sum_{s=1}^{T} x_{i s}^{2}\left\|\hat{\mathbf{f}}_{s}-\mathbf{f}_{s}\right\|_{F}^{2}\right)\right]+ \\
& \|\mathbf{B}\|_{F}\left[\sum_{i=1}^{N}\left(\begin{array}{c}
\frac{1}{T^{2}} \sum_{s=1}^{T} \sum_{s^{\prime}, s \neq s^{\prime}}^{T}\left|x_{i s} x_{i s^{\prime}}\right| \times \\
\left\|\hat{\mathbf{f}}_{s}-\mathbf{f}_{s}\right\|_{F}\left\|\hat{\mathbf{f}}_{s^{\prime}}-\mathbf{f}_{s^{\prime}}\right\|_{F}
\end{array}\right)\right]+ \\
& \sum_{i=1}^{N}\left[\frac{1}{T^{2}} \sum_{s=1}^{T} \sum_{s^{\prime}=1}^{T} x_{i s^{\prime}} v_{i s} \mathbf{f}_{s}^{\prime} \mathbf{B}\left(\hat{\mathbf{f}}_{s^{\prime}}-\mathbf{f}_{s^{\prime}}\right)\right] \\
& =\sum_{i=1}^{5} \tilde{C}_{i} \text {. }
\end{aligned}
$$

We have

$$
\tilde{C}_{1}=\sum_{i=1}^{N}\left(\frac{1}{T^{2}} \sum_{s=1}^{T} \mathbf{f}_{s}^{\prime} \mathbf{B} \mathbf{f}_{s} v_{i s}^{2}\right)=O_{p}\left(N T^{-1}\right)
$$


Also

$$
\begin{aligned}
\tilde{C}_{2} & =\sum_{i=1}^{N}\left(\frac{1}{T^{2}} \sum_{s=1}^{T} \sum_{s^{\prime}, s \neq s^{\prime}}^{T} \mathbf{f}_{s}^{\prime} \mathbf{B} \mathbf{f}_{s^{\prime}} v_{i s} v_{i s^{\prime}}\right) \\
& =\frac{1}{T} \sum_{i=1}^{N}\left[\frac{1}{\sqrt{T}} \sum_{s=1}^{T} v_{i s} \mathbf{f}_{s}^{\prime} \mathbf{B}\left(\frac{1}{\sqrt{T}} \sum_{s^{\prime}, s \neq s^{\prime}}^{T} \mathbf{f}_{s^{\prime}} v_{i s^{\prime}}\right)\right]=O_{p}\left(N T^{-1}\right)
\end{aligned}
$$

and

$$
\begin{aligned}
\tilde{C}_{3} & =\|\mathbf{B}\|_{F} \sum_{i=1}^{N}\left(\frac{1}{T^{2}} \sum_{s=1}^{T} x_{i s}^{2}\left\|\hat{\mathbf{f}}_{s}-\mathbf{f}_{s}\right\|_{F}^{2}\right) \\
& =\|\mathbf{B}\|_{F} \frac{1}{\min (N, T) T} \sum_{i=1}^{N}\left(\frac{\min (N, T)}{T} \sum_{s=1}^{T} x_{i s}^{2}\left\|\hat{\mathbf{f}}_{s}-\mathbf{f}_{s}\right\|_{F}^{2}\right) \\
& =O_{p}\left[N^{2} T^{-1} \min (N, T)^{-1}\right] .
\end{aligned}
$$

Further,

$$
\begin{aligned}
\tilde{C}_{4} & =\|\mathbf{B}\|_{F} \sum_{i=1}^{N}\left(\frac{1}{T^{2}} \sum_{s=1}^{T} \sum_{s^{\prime}, s \neq s^{\prime}}^{T}\left|x_{i s} x_{i s^{\prime}}\right|\left\|\hat{\mathbf{f}}_{s}-\mathbf{f}_{s}\right\|_{F}\left\|\hat{\mathbf{f}}_{s^{\prime}}-\mathbf{f}_{s^{\prime}}\right\|_{F}\right) \\
& \leq\|\mathbf{B}\|_{F} \sum_{i=1}^{N}\left[\left(\frac{1}{T^{2}} \sum_{s=1}^{T} \sum_{s^{\prime}, s \neq s^{\prime}}^{T}\left|x_{i s} x_{i s^{\prime}}\right|\right)^{1 / 2}\right. \\
& \leq\|\mathbf{B}\|_{F} \sum_{i=1}^{N}\left[\left\{\frac{1}{T} \sum_{s=1}^{T} \sum_{s^{\prime}, s \neq s^{\prime}}^{T}\left\|\hat{\mathbf{f}}_{s}-\mathbf{f}_{s}\right\|_{F}^{2}\left\|\hat{\mathbf{f}}_{s^{\prime}}-\mathbf{f}_{s^{\prime}}\right\|_{F}^{2}\right)^{1 / 2}\right] \\
& \left.\leq\|\mathbf{B}\|_{F}\left(\frac{1}{T} \sum_{s=1}^{T}\left[\left\|\hat{\mathbf{f}}_{s}-\mathbf{f}_{s}\right\|_{F}^{T}\left(\frac{1}{T} \sum_{s^{\prime}, s \neq s^{\prime}}^{T}\left\|\hat{\mathbf{f}}_{s}-\mathbf{f}_{s}-\mathbf{f}_{s}\right\|_{s^{\prime}, s \neq s^{\prime}} \|_{F}^{2}\right) x_{i s} x_{i s^{\prime}} \mid\right)^{1 / 2}\right] \sum_{i=1}^{N}\left[\left(\frac{1}{T^{2}} \sum_{s=1}^{T} \sum_{s^{\prime}, s \neq s^{\prime}}^{T}\left|x_{i s} x_{i s^{\prime}}\right|\right)^{1 / 2}\right]\right\},
\end{aligned}
$$

and it follows that $\tilde{C}_{4}=O_{p}\left(\frac{N^{2}}{\min (N, T)}\right)$. Finally, since

$$
\sup _{i}\left\{E\left[\frac{1}{T^{2}} \sum_{s=1}^{T} \sum_{s^{\prime}=1}^{T} x_{i s^{\prime}} v_{i s} \mathbf{f}_{s}^{\prime}\left(\hat{\mathbf{f}}_{s^{\prime}}-\mathbf{f}_{s^{\prime}}\right)\right]^{2}\right\}^{1 / 2}=O_{p}\left(\min (N, T)^{-1}\right),
$$

we have

$$
\tilde{C}_{5}=\|\mathbf{B}\|_{F} \sum_{i=1}^{N}\left[\frac{1}{T^{2}} \sum_{s=1}^{T} \sum_{s^{\prime}=1}^{T} x_{i s^{\prime}} v_{i s} \mathbf{f}_{s}^{\prime}\left(\hat{\mathbf{f}}_{s^{\prime}}-\mathbf{f}_{s^{\prime}}\right)\right]=O_{p}\left[N^{2} \min (N, T)^{-1}\right] .
$$

So overall

$$
\left\|\mathbf{A}_{0}^{\prime}\left(\hat{\mathbf{A}}-\mathbf{A}_{0}\right)\right\|_{F}^{2}=O_{p}\left(\frac{N^{2}}{\min (N, T)}\right) .
$$


and

$$
\left\|\mathbf{A}_{0}^{\prime}\left(\hat{\mathbf{A}}-\mathbf{A}_{0}\right)\right\|_{F}=O_{p}\left(\frac{N}{\sqrt{\min (N, T)}}\right),
$$

proving the required result. 


\section{References}

Acemoglu, D., U. Akcigit, and W. Kerr (2016): "Networks and the macroeconomy: An empirical exploration," NBER Macroeconomics Annual, 30(1), 273-335.

Acemoglu, D., D. Autor, D. Dorn, G. H. Hanson, and B. Price (2016): "Import competition and the great US employment sag of the 2000s," Journal of Labor Economics, 34(S1), S141-S198.

Acemoglu, D., V. M. Carvalho, A. Ozdaglar, and A. Tahbaz-Salehi (2012): "The network origins of aggregate fluctuations," Econometrica, 80(5), 1977-2016.

Ahn, S. C., And A. R. Horenstein (2013): "Eigenvalue ratio test for the number of factors," Econometrica, 81(3), 1203-1227.

BAI, J. (2003): "Inferential theory for factor models of large dimensions," Econometrica, 71(1), $135-171$.

BAI, J., AND S. NG (2002): "Determining the number of factors in approximate factor models," Econometrica, 70(1), 191-221.

(2006): "Evaluating latent and observed factors in macroeconomics and finance," Journal of Econometrics, 131(1-2), 507-537.

(2008): "Large dimensional factor analysis," Foundations and Trends@ in Econometrics, 3(2), 89-163.

Bailey, N., S. Holly, and M. H. Pesaran (2016): "A Two-Stage Approach to SpatioTemporal Analysis with Strong and Weak Cross-Sectional Dependence," Journal of Applied Econometrics, 31(1), 249-280.

Bailey, N., G. Kapetanios, and M. H. Pesaran (2016): "Exponent of Cross Sectional Dependence: Estimation and Inference," Journal of Applied Econometrics, 31(6), 929-960.

(2018): "Exponent of Cross-sectional Dependence for Residuals," USC-INET Research Paper No. 18-15, Available at SSRN: https://papers.ssrn.com/sol3/papers . cfm? abstract_id=3256904.

Bailey, N., M. H. Pesaran, and L. V. Smith (2018): "A Multiple Testing Approach to the Regularisation of Large Sample Correlation Matrices," Journal of Econometrics (forthcoming).

Bickel, P. J., And E. Levina (2008): "Covariance regularization by thresholding," The Annals of Statistics, 36(6), 2577-2604.

Brownlees, C., and G. Mesters (2018): "Detecting Granular Time Series in Large Panels," Version dated to July 13, 2018. Available at SSRN: https://ssrn. com/abstract=3040636.

Cai, T., And W. LiU (2011): "Adaptive Thresholding for Sparse Covariance Matrix Estimation," Journal of American Statistical Association, 106(494), 672-684. 
Cesa-Bianchi, A., M. H. Pesaran, and A. Rebucci (2018): "Uncertainty and Economic Activity: A Multi-Country Perspective," Discussion paper, National Bureau of Economic Research, Working Paper 24325.

Chudik, A., G. Kapetanios, and M. H. Pesaran (2018): "A One Covariate at a Time, Multiple Testing Approach to Variable Selection in High-Dimensional Linear Regression Models," Econometrica, 86(4), 1479-1512.

Chudik, A., M. H. Pesaran, and E. Tosetti (2011): "Weak and strong cross-section dependence and estimation of large panels," The Econometrics Journal, 14(1), C45-C90.

Dungey, M., And V. Volkov (2018): "R\&D and wholesale trade are critical to the economy: Identifying dominant sectors from economic networks," Economics Letters, 162, 81-85.

El Karoui, N. (2008): "Operator norm consistent estimation of large-dimensional sparse covariance matrices," The Annals of Statistics, 36(6), 2717-2756.

Foerster, A. T., P.-D. G. Sarte, and M. W. Watson (2011): "Sectoral versus aggregate shocks: A structural factor analysis of industrial production," Journal of Political Economy, 119(1), 1-38.

Gaibulloev, K., T. Sandler, and D. Sul (2013): "Common drivers of transnational terrorism: Principal component analysis," Economic Inquiry, 51(1), 707-721.

Greenaway-McGrevy, R., N. C. Mark, D. Sul, and J.-L. Wu (2018): "Identifying exchange rate common factors," International Economic Review (forthcoming), https:// doi.org/10.1111/iere.12334.

Holly, S., M. H. Pesaran, and T. Yamagata (2010): "A spatio-temporal model of house prices in the USA," Journal of Econometrics, 158(1), 160-173.

Mohaddes, K., and M. Raissi (2018): "Compilation, Revision and Updating of the Global VAR (GVAR) Database, 1979Q2-2016Q4," (mimeo).

Parker, J., and D. Sul (2016): "Identification of Unknown Common Factors: Leaders and Followers," Journal of Business \& Economic Statistics, 34(2), 227-239.

Pesaran, M. H., and C. F. Yang (2016): "Econometric Analysis of Production Networks with Dominant Units," Discussion paper, USC-INET Research Paper No. 16-25, Available at SSRN: https://ssrn. com/abstract=2851148.

Soofi-Siavash, S. (2018): "Dominant U.S. Manufacturing Sectors: A Factor Model Analysis," (mimeo). Available at https://sites.google.com/site/sorooshsoofisiavash/ research.

YAnG, C. F. (2018): "Common Factors and Spatial Dependence: An Application to US House Prices," Available at https://sites.google.com/site/cynthiafanyang/research. 


\title{
Online Supplement to
}

\section{A Residual-based Threshold Method for Detection of Units that are Too Big to Fail in Large Factor Models}

by

\author{
G. Kapetanios, M. H. Pesaran and S. Reese
}

November 2018

This online supplement contains additional theoretical, simulation and empirical results that complement the main paper. It is composed of five sections. Section S1 gives a more detailed description of the steps required to implement the various variants of the basic $\sigma^{2}$ thresholding method proposed in the paper. A summary of other approaches proposed in the literature for the detection of dominant units is given in Section S2. The finite sample performance of the different variants of the $\sigma^{2}$ thresholding (that are not considered in Section 6 of the paper) is discussed in Section S3. Two additional $\sigma^{2}$ thresholding schemes, based on the difference and the ratio of two successive ordered error variance estimates, are considered in Section S4 and their small sample properties investigated using Monte Carlo simulations. Finally, Sections S5 and S6 report simulation and empirical results using an unmodified version of BM procedure.

\section{S1 Variants of the basic $\sigma^{2}$ thresholding Methods}

This section provides a step by step description of the various refinements of the basic $\sigma^{2}$ thresholindg advanced in Section 5 of the paper. Let $\mathbf{x}_{i}$ be the $T \times 1$ vector of observations on the $i$-th unit in the panel, and $\mathbf{X}=\left(\mathbf{x}_{1}, \mathbf{x}_{2}, \ldots, \mathbf{x}_{N}\right)$ be the $T \times N$ matrix of observations on all the $N$ units in the panel. Suppose that $p \leq p_{\max }$, where $p_{\max }$ is selected a priori to be sufficiently large. Denote by $\mathbf{X}_{a}^{*}$ the $T \times r$ matrix containing all dominant units that have been identified at a given step of the two algorithms described below. Analogously, let the $T \times N_{1}$ matrix $\mathbf{X}_{b}^{*}=\left(\mathbf{x}_{b, 1} ; \ldots ; \mathbf{x}_{b, N_{1}}\right)$ contain observations for the $N_{1}=N-r$ remaining cross-section units that have not been identified as dominant. Furthermore, let

$$
\mathbf{M}_{\mathbf{X}_{a}^{*}}=\left\{\begin{array}{c}
\mathbf{I}_{T}, \quad \text { if } r=0, \\
\mathbf{I}_{T}-\mathbf{X}_{a}^{*}\left(\mathbf{X}_{a}^{* \prime} \mathbf{X}_{a}^{*}\right)^{-1} \mathbf{X}_{a}^{* \prime}, \quad \text { if } r>0 .
\end{array}\right.
$$

Given the sequential nature of the two algorithms described below, the values of $r, N_{1}, \mathbf{X}_{a}^{*}$ and $\mathbf{X}_{b}^{*}$ and the dimensions of the latter two matrices change as the algorithm proceeds. Furthermore, $\mathbf{X}_{a}^{*}$ and $\mathbf{X}_{b}^{*}$ represent an estimated partition of the data into dominant and non-dominant units which is to be distinguish from the true partition $\mathbf{X}=\left(\mathbf{X}_{a} ; \mathbf{X}_{b}\right)$.

\section{Algorithm 4 (Sequential $\sigma^{2}$ thresholding)}

1. Set $r=0$. 
2. Compute $\hat{\mathbf{F}}=\frac{1}{\sqrt{N}} \mathbf{M}_{\mathbf{X}_{a}^{*}} \mathbf{X}_{b}^{*} \hat{\mathbf{Q}}$, where $\hat{\mathbf{Q}}$ is the $N \times\left(p_{\max }-r\right)$ matrix whose columns are the orthonormal eigenvectors of $\mathbf{X}_{b}^{* \prime} \mathbf{M}_{\mathbf{X}_{a}^{*}} \mathbf{X}_{b}^{*}$, such that $N^{-1} \hat{\mathbf{Q}}^{\prime} \hat{\mathbf{Q}}=\mathbf{I}_{p_{\max }}$. For each $i=1$, compute $\hat{\mathbf{a}}_{i}, \hat{v}_{i t}$ and $\hat{\sigma}_{i T}^{2}$ to be the OLS estimator, residual and residual variance of the regression of $\mathbf{x}_{b, i}^{*}$ on $\hat{\mathbf{F}}$, namely

$$
\begin{aligned}
\hat{\mathbf{a}}_{i} & =\left(\hat{\mathbf{F}}^{\prime} \hat{\mathbf{F}}\right)^{-1} \hat{\mathbf{F}}^{\prime} \mathbf{x}_{b, i}^{*}, \\
\hat{\mathbf{v}}_{i} & =\left(\hat{v}_{i 1}, \hat{v}_{i 2}, \ldots, \hat{v}_{i T}\right)^{\prime}=\mathbf{M}_{\hat{F}} \mathbf{x}_{b, i}^{*}=\left[\mathbf{I}_{T}-\hat{\mathbf{F}}\left(\hat{\mathbf{F}}^{\prime} \hat{\mathbf{F}}\right)^{-1} \hat{\mathbf{F}}^{\prime}\right] \mathbf{x}_{b, i}^{*}, \\
\hat{\sigma}_{i T}^{2} & =T^{-1} \mathbf{x}_{b, i}^{* \prime} \mathbf{M}_{\hat{\mathbf{F}}^{\prime}} \mathbf{x}_{b, i}^{*} .
\end{aligned}
$$

3. Compute

$$
\hat{\eta}_{i N}^{2}=\frac{\hat{\mathbf{a}}_{i}^{\prime} \hat{\mathbf{A}}^{\prime} \tilde{\boldsymbol{\Sigma}}_{v} \hat{\mathbf{A}} \hat{\mathbf{a}}_{i}}{N},
$$

for every $i=1,2, \ldots, N$ where $\tilde{\boldsymbol{\Sigma}}_{v}$ is the multiple testing estimator of $\boldsymbol{\Sigma}_{v}$ by Bailey, Pesaran, and Smith (2018), as described in Section 4.2 of the main paper. If for all $i$,

$$
\hat{\sigma}_{i T}^{2}>\frac{2 \hat{\eta}_{i N}^{2} \log T}{N},
$$

then stop the algorithm and conclude that there are $\hat{m}=r$ dominant units whose identities are given by the indices of the columns in $\mathbf{X}$ that coincide with columns in $\mathbf{X}_{a}^{*}$. Otherwise, proceed to step 4 .

4. Let $i^{*}=\arg \min _{i} \hat{\sigma}_{i}^{2}$. Update $\mathbf{X}_{a}^{*}=\left(\mathbf{X}_{a}^{*} ; \mathbf{x}_{b, i^{*}}\right)$ and eliminate $\mathbf{x}_{b, i^{*}}$ from $\mathbf{X}_{b}^{*}$. Update, $r:=r+1$ and $N_{1}:=N_{1}-1$ and return to step 2.

\section{Algorithm 5 (Sequential-MT $\sigma^{2}$ thresholding )}

1. Set $r=0$.

2. Compute $\hat{\mathbf{F}}=\frac{1}{\sqrt{N}} \mathbf{M}_{\mathbf{X}_{a}^{*}} \mathbf{X}_{b}^{*} \hat{\mathbf{Q}}$, where $\hat{\mathbf{Q}}$ is the $N \times\left(p_{\max }-r\right)$ matrix whose columns are the orthonormal eigenvectors of $\mathbf{X}_{b}^{* \prime} \mathbf{M}_{\mathbf{X}_{a}^{*}} \mathbf{X}_{b}^{*}$, such that $N^{-1} \hat{\mathbf{Q}}{ }^{\prime} \mathbf{Q}=\mathbf{I}_{p_{\max }}$. For each $i=1$, compute $\hat{\mathbf{a}}_{i}, \hat{v}_{i t}$ and $\hat{\sigma}_{i T}^{2}$ to be the OLS estimator, residual and residual variance of the regression of $\mathbf{x}_{b, i}^{*}$ on $\hat{\mathbf{F}}$, namely

$$
\begin{aligned}
\hat{\mathbf{a}}_{i} & =\left(\hat{\mathbf{F}}^{\prime} \hat{\mathbf{F}}\right)^{-1} \hat{\mathbf{F}}^{\prime} \mathbf{x}_{b, i}^{*}, \\
\hat{\mathbf{v}}_{i} & =\left(\hat{v}_{i 1}, \hat{v}_{i 2}, \ldots, \hat{v}_{i T}\right)^{\prime}=\mathbf{M}_{\hat{\mathbf{F}}} \mathbf{x}_{b, i}^{*}=\left[\mathbf{I}_{T}-\hat{\mathbf{F}}\left(\hat{\mathbf{F}}^{\prime} \hat{\mathbf{F}}\right)^{-1} \hat{\mathbf{F}}^{\prime}\right] \mathbf{x}_{b, i}^{*}, \\
\hat{\sigma}_{i T}^{2} & =T^{-1} \mathbf{x}_{b, i}^{* \prime} \mathbf{M}_{\hat{\mathbf{F}}} \mathbf{x}_{b, i}^{*} .
\end{aligned}
$$

3. Compute

$$
\hat{\eta}_{i N}^{2}=\frac{\hat{\mathbf{a}}_{i}^{\prime} \hat{\mathbf{A}}^{\prime} \tilde{\boldsymbol{\Sigma}}_{v} \hat{\mathbf{A}} \hat{\mathbf{a}}_{i}}{N}
$$


for every $i=1,2, \ldots, N$ where $\tilde{\boldsymbol{\Sigma}}_{v}$ is the multiple testing estimator of $\boldsymbol{\Sigma}_{v}$ by Bailey, Pesaran, and Smith (2018), as described in Section 4.2 of the main paper. If for all $i$,

$$
\hat{\sigma}_{i T}^{2}>\frac{2 \hat{\eta}_{i N}^{2} \log T}{N}
$$

then stop the algorithm and conclude that there are $\hat{m}=r$ dominant units whose identities are given by the indices of the columns in $\mathbf{X}$ that coincide with columns in $\mathbf{X}_{a}^{*}$. Otherwise, proceed to step 4 .

4. Let $i^{*}=\arg \min _{i} \hat{\sigma}_{i}^{2}$. For each $j=1, \ldots i^{*}-1, i^{*}+1, \ldots, N_{1}$ estimate the model

$$
\mathbf{M}_{\mathbf{X}_{a}^{*}} \mathbf{x}_{b, j}=\mathbf{M}_{\mathbf{X}_{a}^{*}} \mathbf{x}_{b, i^{*}} \gamma_{j}^{*}+\mathbf{f}_{t}^{* \prime} \mathbf{a}_{j}^{*}+\mathbf{v}_{j}
$$

where $\mathbf{f}_{t}^{*}$ is a $p_{\max }-r-1$ vector of unobserved factors which we estimate as in step 2 but using $\mathbf{M}_{\mathbf{X}_{a}^{*}} \mathbf{X}_{b,-i^{*}}^{*}$ with $\mathbf{X}_{b,-i^{*}}=\left(\mathbf{x}_{b, 1} ; \ldots ; \mathbf{x}_{b, i^{*}-1} ; \mathbf{x}_{b, i^{*}+1} ; \ldots ; \mathbf{x}_{b, N_{1}}\right)$ instead of $\mathbf{M}_{\mathbf{X}_{a}^{*}} \mathbf{X}_{b}^{*}$.

5. Apply individual significance tests to the $N_{1}-1$ estimated slope parameters $\hat{\gamma}_{1}^{*}, \ldots, \hat{\gamma}_{i^{*}-1}^{*}, \hat{\gamma}_{i^{*}+1}^{*}, \ldots, \hat{\gamma}_{N_{1}}^{*}$ using the critical value $\Phi^{-1}\left[1-\frac{\pi}{2\left(N_{1}-1\right)}\right]$ with $\Phi^{-1}(\cdot)$ denoting the inverse normal CDF, and $\pi$ is set to 0.01 .

6. Let $M$ denote the number of rejections among these $N_{1}-1$ tests. If $\log (M) / \log (N) \leq 1 / 2$, stop and conclude that there are $\hat{m}=r$ dominant units whose identities are given by the indices of the columns in $\mathbf{X}$ that coincide with the columns of $\mathbf{X}_{a}^{*}$. Otherwise proceed to step 7.

7. Update $\mathbf{X}_{a}^{*}=\left(\mathbf{X}_{a}^{*} ; \mathbf{x}_{b, i^{*}}\right)$ and eliminate $\mathbf{x}_{b, i^{*}}$ from $\mathbf{X}_{b}^{*}$. Update, $r:=r+1$ and $N_{1}:=N_{1}-1$ and return to step 2 .

\section{S2 Dominant unit detection procedures proposed in the literature}

\section{S2.1 Brownlees and Mesters (BM) procedure}

The model considered in (Brownlees and Mesters, 2018, BM in the following) has an equivalent reformulation of our dominant unit model, formally given by

$$
\begin{aligned}
\underset{m \times 1}{\mathbf{x}_{t a}} & =\mathbf{f}_{t}, \\
\underset{n \times 1}{\mathbf{x}_{t b}} & =\mathbf{B} \mathbf{x}_{t a}+\mathbf{u}_{t},
\end{aligned}
$$

where the covariance matrix of $\mathbf{f}_{t}$ may be any positive definite matrix. Brownlees and Mesters (2018) also allow for the presence of unobserved common factors, but we will be abstracting from such factors to simplify the exposition.

The number of dominant units ${ }^{19}$ and their identities are estimated from the precision matrix (i.e. the inverse covariance matrix) of the observed data X. Formally, let

$$
\hat{\mathbf{K}}=\left(T^{-1} \mathbf{X}^{\prime} \mathbf{X}-\overline{\mathbf{x x}}^{\prime}\right)^{-1},
$$

\footnotetext{
${ }^{19}$ Brownlees and Mesters (2018) employ the term granular shocks instead of dominant units.
} 
where $\overline{\mathbf{x}}=\left(\bar{x}_{1} ; \ldots ; \bar{x}_{N}\right)^{\prime}$, and $\bar{x}_{i}=T^{-1} \sum_{t=1}^{T} x_{i t}$. Additionally, let $\hat{\mathbf{K}}=\left(\begin{array}{lll}\hat{\mathbf{k}}_{1} & \ldots & \hat{\mathbf{k}}_{N}\end{array}\right)$. BM then compute $\hat{\kappa}_{i}=\left\|\hat{\mathbf{k}}_{i}\right\|, i=1,2, \ldots, N$, where $\left\|\hat{\mathbf{k}}_{i}\right\|=\sqrt{\hat{\mathbf{k}}_{i}^{\prime} \hat{\mathbf{k}}_{i}}$. These $N$ vector norms are then ordered in a descending manner, denoted as $\hat{\kappa}_{(1)}, \hat{\kappa}_{(2)}, \ldots, \hat{\kappa}_{(N)}$. The estimated number of dominant units is then

$$
\hat{m}=\underset{j=1,2, \ldots, N-1}{\arg \max } \frac{\hat{\kappa}_{(j)}}{\hat{\kappa}_{(j+1)}},
$$

and the dominant units are determined as columns with the norms $\hat{\kappa}_{(1)}, \hat{\kappa}_{(2)}, \ldots, \hat{\kappa}_{(\hat{m})}$. Monte Carlo simulations and empirical applications in the main paper employ a slight modification of this procedure, also used in Section 6 of Brownlees and Mesters (2018), whereby the above maximimzation problem is solved with respect to the first $N / 2$ ratios instead of all $N-1$ ratios. Supplementary simulation results obtained without this modification are reported in Sections S5 and S6.

BM detection method is subject to two main shortcomings. First, estimation of the precision matrix requires $T>N$. Second, by construction the estimated number of dominant units is at least one. Consequently, it is impossible to use the BM procedure to investigate whether there is in fact any dominant unit in the panel data set under consideration. As an illustration consider the simple factor specification

$$
x_{i t}=\beta_{i} f_{t}+u_{i t}
$$

where $f_{t} \sim(0,1)$ is the common factor, $\beta_{i}$ is the factor loading with sup $\left|\beta_{i}\right|<K$, and $u_{i t}$ is the unit-specific component which we assume to be $\operatorname{IID}\left(0, \sigma^{2}\right)$ over all $i$ and $t, i=1,2, \ldots, N ; t=$ $1,2, \ldots, T$. Assuming that $\sigma^{2}>0$ ensures that there is no dominant unit in this model. Let $\mathbf{x}_{t}=\left(x_{1 t}, x_{2 t}, \ldots, x_{N t}\right)^{\prime}, \boldsymbol{\beta}=\left(\beta_{1}, \beta_{2}, \ldots, \beta_{N}\right)$, and $\mathbf{u}_{t}=\left(u_{1 t}, u_{2 t}, \ldots, u_{N t}\right)$, and write $(69)$ as

$$
\mathbf{x}_{t}=\boldsymbol{\beta} f_{t}+\mathbf{u}_{t},
$$

and note that

$$
\operatorname{Cov}\left(\mathbf{x}_{t}\right)=\boldsymbol{\Sigma}=\boldsymbol{\beta} \boldsymbol{\beta}^{\prime}+\sigma^{2} \mathbf{I}_{N}
$$

Then,

$$
\begin{aligned}
& \mathbf{K}=\left(\begin{array}{llll}
\mathbf{k}_{1} & \mathbf{k}_{2} & \cdots & \mathbf{k}_{N}
\end{array}\right) \\
& =\boldsymbol{\Sigma}^{-1}=\left(\sigma^{2} \mathbf{I}_{N}+\boldsymbol{\beta} \boldsymbol{\beta}^{\prime}\right)^{-1}=\frac{1}{\sigma^{2}}\left(\mathbf{I}_{N}-\frac{\boldsymbol{\delta} \boldsymbol{\delta}^{\prime}}{1+\boldsymbol{\delta}^{\prime} \boldsymbol{\delta}}\right),
\end{aligned}
$$

where $\boldsymbol{\delta}=\left(\delta_{1}, \delta_{2}, \ldots, \delta_{N}\right)^{\prime}$ and $\delta_{i}=\beta_{i} / \sigma$. Then, it is easily seen that

$$
\left\|\mathbf{k}_{i}\right\|^{2}=\frac{1}{\sigma^{4}}\left[\left(1-\frac{\boldsymbol{\delta} \boldsymbol{\delta}^{\prime}}{1+\boldsymbol{\delta}^{\prime} \boldsymbol{\delta}}\right)^{2}+\frac{\delta_{i}^{2} \sum_{j \neq i}^{N} \delta_{j}^{2}}{\left(1+\boldsymbol{\delta}^{\prime} \boldsymbol{\delta}\right)^{2}}\right] .
$$

Suppose that $\boldsymbol{\beta}^{\prime} \boldsymbol{\beta}=\sigma^{2} \boldsymbol{\delta}^{\prime} \boldsymbol{\delta}=\Theta\left(N^{\alpha}\right)$, with $\alpha=1$, signifying $f_{t}$ to be strong. Then, $\left\|\mathbf{k}_{i}\right\|^{2}=$ $\frac{1}{\sigma^{4}} \delta_{i}^{2} \frac{\delta \boldsymbol{\delta}^{\prime}}{\left(1+\boldsymbol{\delta}^{\prime} \boldsymbol{\delta}\right)^{2}}$, and hence, as $N \rightarrow \infty$,

$$
\lim _{N \rightarrow \infty} \frac{\left\|\mathbf{k}_{i}\right\|}{\left\|\mathbf{k}_{s}\right\|}=\frac{\left|\delta_{i}\right|}{\left|\delta_{s}\right|}=\frac{\left|\beta_{i}\right|}{\left|\beta_{s}\right|}
$$


and $\left\|\mathbf{k}_{i}\right\|$ is maximized for the unit with the largest factor loading in absolute value.

The same result holds if we allow the variance of $u_{i t}$ to vary over $i$. In such a case the relevant measure is $\left|\beta_{i}\right| / \sigma_{i}^{2}$, where $\sigma_{i}^{2}=V\left(u_{i t}\right)$, with $0<\sigma_{i}^{2}<K$. Thus, the column norms of the concentration matrix measure the relative importance of the common factors for the units in the panel, and is not informative about the importance of the unit for the rest of the units in the panel (network).

\section{S2.2 Parker and Sul (PS) procedure}

The dominant leader framework of (Parker and Sul, 2016, henceforth PS) is primarily aimed at investigating whether a time series external to the dataset at hand is one of the latent factors driving the observed data. However, this framework can be represented in terms of the model (67)-(68) by simply including the potential dominant unit(s) into the dataset (see also Parker and Sul, 2016, p.229). The dominant leader framework also deals with approximate dominant leaders which will not be considered here.

The key idea of PS is whether a known potential dominant unit can replace one of the factor estimates obtained from the factor model representation of the dominant unit model. If so, then this candidate unit is identified as dominant.

PS assume a priori knowledge of a fixed number $r$ of potential dominant units, denoted as $\mathbf{G}=\left(\mathbf{g}_{1}, \ldots, \mathbf{g}_{r}\right)$. Each time series in the dataset is standardized and the true number of factors in the data is determined. In order to avoid a subjective choice, we let $p_{\max }=\hat{\#}(\mathbf{X})$ where $\hat{\#}(\mathbf{X})$ denotes the number of factors in $\mathbf{X}$ minimizing the the $I C_{p 2}$ criterion of Bai and $\mathrm{Ng}$ (2002). ${ }^{20}$ Subsequently, the factor estimates $\hat{\mathbf{F}}$ are obtained as $\sqrt{T}$ times the eigenvectors corresponding to the $p_{\max }$ largest eigenvalues of $N^{-1} \mathbf{X} \mathbf{X}^{\prime}$. Now, for each potential dominant unit $\mathbf{g}_{\ell}, \ell=1, \ldots, p_{\max }$, Parker and Sul consider the $p_{\max }$ regression models

$$
\begin{aligned}
x_{i t} & =\gamma_{i, 1} g_{t, \ell}+\alpha_{i, 2} \hat{f}_{t, 2}+\ldots+\alpha_{i, p_{\max }} \hat{f}_{t, p_{\max }}+\eta_{i t}^{(1)}, \\
x_{i t} & =\alpha_{i, 1} \hat{f}_{t, 1}+\gamma_{i, 2} g_{t, \ell}+\ldots+\alpha_{i, p_{\max }} \hat{f}_{t, p_{\max }}+\eta_{i t}^{(2)}, \\
& \vdots \\
x_{i t} & =\alpha_{i, 1} \hat{f}_{t, 1}+\alpha_{i, 2} \hat{f}_{t, 2}+\ldots+\gamma_{i, p_{\max }} g_{t, \ell}+\eta_{i t}^{\left(p_{\max }\right)},
\end{aligned}
$$

for $i=1,2, \ldots, N$. Let $\hat{\mathbf{H}}^{(1)}=\left(\hat{\eta}_{1 t}^{(1)}, \ldots, \hat{\eta}_{N t}^{(1)}\right), \ldots, \hat{\mathbf{H}}^{\left(p_{\max }\right)}=\left(\hat{\eta}_{1 t}^{\left(\hat{m}_{0}\right)}, \ldots, \hat{\eta}_{N t}^{\left(p_{\max }\right)}\right)$ denote the OLS residuals of the $p_{\max }$ regression models above. If at least one among $\hat{\#}\left(\hat{\mathbf{H}}^{(1)}\right), \ldots, \hat{\#}\left(\hat{\mathbf{H}}^{\left(p_{\max }\right)}\right)$ is equal to zero then $\mathbf{g}_{\ell}$ is considered as a dominant unit.

PS suggest a further step if any of the units in the dataset is selected as dominant. For each unit $\mathbf{x}_{i}$, the authors consider the $p_{\max }$ regression models

$$
\begin{aligned}
\hat{f}_{t, 1} & =c_{1, i}^{(1)} x_{i t}+c_{2, i}^{(1)} \hat{f}_{t, 2}+\ldots+c_{p_{\max }, i}^{(1)} \hat{f}_{t, p_{\max }}+\zeta_{t}^{(1)}, \\
\hat{f}_{t, 2} & =c_{1, i}^{(2)} \hat{f}_{t, 1}+c_{2, i}^{(2)} x_{i t}+\ldots+c_{p_{\max }, i}^{(2)} \hat{f}_{t, p_{\max }}+\zeta_{t}^{(2)}, \\
\vdots & \\
\hat{f}_{t, \hat{m}_{0}} & =c_{1, i}^{\left(p_{\max }\right)} \hat{f}_{t, 1}+c_{2, i}^{\left(p_{\max }\right)} \hat{f}_{t, 2}+\ldots+c_{p_{\max , i}}^{\left(p_{\max }\right)} x_{i t}+\zeta_{t}^{\left(p_{\max }\right)} .
\end{aligned}
$$

\footnotetext{
${ }^{20}$ In application of the Bai-Ng selection procedure, we set the maximum number of factors to 10.
} 
The coefficients of determination $R_{1, i}^{2}, \ldots, R_{p_{\max , i}}^{2}$ for these $p_{\max }$ regression equations are obtained. Having done this for every $i=1,2, \ldots, N$, the $R^{2}$ values for the first model above, denoted by $R_{1,1}^{2}, \ldots, R_{1, N}^{2}$, are ordered in a descending manner. The units with the coefficient

of determination $R_{1,(1)}^{2}, \ldots, R_{1,\left(r^{*}\right)}^{2}$ are chosen as $r^{*}$ potential dominant units. This procedure is repeated for the remaining $p_{\max }-1$ models as set out above, providing in total $r=r^{*} p_{\max }$ potential dominant units (duplicates included). A guideline for the choice of $r^{*}$ is "[...] around $10 \%$ of the size of $N . "$ (Parker and Sul, 2016, p.232).

The PS procedure is subject to two limitations. First, Parker and Sul (2016, p.230) acknowledge that treating all units in the sample as potential dominant units may lead to a non-negligible probability of making a Type I error. However, this problem is not solved by restricting the number of potential dominant units to $10 \%$ of the number of cross-sections. Second, the performance of the procedure depends crucially on the choice of $m$, the number of factors, and how well it is estimated. If $m$ is underestimated not all true dominant units may be chosen. If it is overestimated, non-dominant units may falsely be identified as dominant.

\section{S3 Finite sample performance of alternative $\sigma^{2}$ thresh- olding methods}

As discussed in Section 5 of the paper, it is possible to apply certain refinements to the $\sigma^{2}$ thresholding method in order to improve its finite sample properties. Our preference for the sequential-MT $\sigma^{2}$ thresholding is based on its finite sample performance relative to a number of other modified versions of the basic method. This section provides simulation results to support our choice.

The $\sigma^{2}$ thresholding variations considered are as follows:

1. $\sigma^{2}$ thresholding, as described by Algorithm 1 in the paper.

2. $\mathrm{S}-\sigma^{2}$ thresholding, as described by Algorithm 4 given above, or Algorithm 2 in the paper.

3. Sequential-MT $\sigma^{2}$ thresholding with an alternative threshold. This method coincides with Algorithm 5 except for the application of the threshold specified in Section A.3 for the $\sigma^{2}$ thresholding step.

We conduct simulation experiments identical to those in Section 6 of the paper, and report the performance of $\sigma^{2}$ thresholding, as discussed in Section 4.2, as well as $\mathrm{S}-\sigma^{2}$ thresholding, and the SMT $-\sigma^{2}$ thresholding with an alternative scaling, as set out above. As before, our performance measures are (a) the percent probability of correctly determining only the true dominant units, and (b) the average number of units falsely selected as dominant.

Tables S3.1 and S3.2 report results for the case where there is no dominant unit. The performance of the four measures considered differs only with respect to whether they involve a multiple testing hurdle or not. Algorithms that include this extra step perform better, especially when the DGP includes an external factor. This observation suggests that the multiple testing hurdle makes a noticeable contribution to minimizing the probability of falsely discovering a dominant unit.

Noticeable differences between all four algorithms begin to emerge when the number of dominant units is at least equal to one. As reported in Table S3.3, the performance of $\sigma^{2}$ 
thresholding declines considerably when the total number of factors, both dominant units and external factors, is larger than one. This problem is somewhat mitigated if one considers $\mathrm{S}-\sigma^{2}$ thresholding. However, this method often fails to correctly detect the true dominant units when $T>N$, and there are external factors affecting the observations. The multiple testing hurdle in SMT $-\sigma^{2}$ thresholding addresses this problem and leads to substantial performance gains, thus making it our method of choice. Finally, considering the alternative scaling of the threshold value (variant 3 above) leads to ambiguous results: improved performance is obtained when $N$ is much larger than $T$, in the case where there are two dominant units and at least one external factor. However, the opposite result is obtained if $N$ is only twice as large as $T$. For this reason, we discard the alternative thresholding even though it certainly has benefits in samples where $N-T$ is sufficiently large.

Summary results for the number of units falsely detected as dominant are reported in Table S3.4, and suggest that all the four methods generally perform well in this respect and do not severely overestimate the number of dominant units. However, there is some evidence of false discovery when $k_{0}=2$, and $N$ and $T$ are relatively small.

Qualitatively similar results are obtained when we consider Monte Carlo designs with weakly dominant units. Table S3.5 summarizes the results when $\alpha=0.8$. As can be seen these results are comparable to those reported in S3.3 for $\alpha=1$, the main difference being that with weakly dominant units the probability of correctly determining the true dominant units is lower. Additionally, all $\sigma^{2}$ thresholding versions suffer from performance losses if $N$ is too large relative to $T$. This is to be expected since the fraction of cross section units that are unaffected by dominant units increases in $N$. Finally, Table S3.6 reports the empirical frequency of false discoveries in the case of weakly dominant units. Once again the results are similar to those obtained for $\alpha=1$. 
Table S3.1: Empirical frequency of correctly identifying the absence of a dominant unit

\begin{tabular}{|c|c|c|c|c|c|c|c|c|c|}
\hline \multirow{2}{*}{\multicolumn{6}{|c|}{$\begin{array}{c}\sigma^{2} \text { thresholding } \\
k_{0}=0\end{array}$}} & \multicolumn{4}{|c|}{$\mathrm{S}-\sigma^{2}$} \\
\hline & & & & & & \multicolumn{4}{|c|}{$k_{0}=0$} \\
\hline $\mathrm{N} \backslash \mathrm{T}$ & 60 & 110 & 210 & 250 & $\mathrm{~N} \backslash \mathrm{T}$ & 60 & 110 & 210 & 250 \\
\hline 50 & 96.1 & 93.5 & 93.6 & 92.6 & 50 & 96.1 & 93.5 & 93.6 & 92.6 \\
\hline 100 & 99.0 & 98.1 & 96.3 & 96.6 & 100 & 99.0 & 98.1 & 96.3 & 96.6 \\
\hline 200 & 99.8 & 99.6 & 99.5 & 98.9 & 200 & 99.8 & 99.6 & 99.5 & 98.9 \\
\hline 500 & 100 & 100 & 100 & 100 & 500 & 100 & 100 & 100 & 100 \\
\hline \multicolumn{6}{|c|}{$k_{0}=1$} & \multicolumn{4}{|c|}{$k_{0}=1$} \\
\hline $\mathrm{N} \backslash \mathrm{T}$ & 60 & 110 & 210 & 250 & $\mathrm{~N} \backslash \mathrm{T}$ & 60 & 110 & 210 & 250 \\
\hline 50 & 60.9 & 47.8 & 41.5 & 40.4 & 50 & 60.9 & 47.8 & 41.5 & 40.4 \\
\hline 100 & 89.8 & 80.8 & 71.4 & 68.9 & 100 & 89.8 & 80.8 & 71.4 & 68.9 \\
\hline 200 & 99.3 & 98.6 & 97.6 & 97.0 & 200 & 99.3 & 98.6 & 97.6 & 97.0 \\
\hline 500 & 100 & 100 & 100 & 99.9 & 500 & 100 & 100 & 100 & 99.9 \\
\hline \multicolumn{6}{|c|}{$k_{0}=2$} & \multicolumn{4}{|c|}{$k_{0}=2$} \\
\hline $\mathrm{N} \backslash \mathrm{T}$ & 60 & 110 & 210 & 250 & $\mathrm{~N} \backslash \mathrm{T}$ & 60 & 110 & 210 & 250 \\
\hline 50 & 35.1 & 24.0 & 17.1 & 15.8 & 50 & 35.1 & 24.0 & 17.1 & 15.8 \\
\hline 100 & 77.3 & 60.4 & 43.9 & 39.7 & 100 & 77.3 & 60.4 & 43.9 & 39.7 \\
\hline 200 & 98.3 & 95.7 & 90.9 & 89.3 & 200 & 98.3 & 95.7 & 90.9 & 89.3 \\
\hline \multirow[t]{2}{*}{500} & 100 & 100 & 100 & 99.8 & 500 & 100 & 100 & 100 & 99.8 \\
\hline & \multicolumn{4}{|c|}{$\begin{array}{l}\mathrm{SMT}-\sigma^{2} \\
k_{0}=0\end{array}$} & \multicolumn{5}{|c|}{$\begin{array}{c}\text { SMT }-\sigma^{2}, \text { alternative scaling } \\
k_{0}=0\end{array}$} \\
\hline $\mathrm{N} \backslash \mathrm{T}$ & 60 & $\begin{array}{r}\kappa_{0} \\
110\end{array}$ & $\begin{array}{l}=0 \\
210\end{array}$ & 250 & $\mathrm{~N} \backslash \mathrm{T}$ & 60 & $\begin{array}{r}\kappa_{0} \\
110\end{array}$ & 210 & 250 \\
\hline 50 & 100 & 100 & 100 & 100 & 50 & 100 & 100 & 100 & 100 \\
\hline 100 & 100 & 100 & 100 & 100 & 100 & 100 & 100 & 100 & 100 \\
\hline 200 & 100 & 100 & 100 & 100 & 200 & 100 & 100 & 100 & 100 \\
\hline 500 & 100 & 100 & 100 & 100 & 500 & 100 & 100 & 100 & 100 \\
\hline \multicolumn{6}{|c|}{$k_{0}=1$} & \multicolumn{4}{|c|}{$k_{0}=1$} \\
\hline $\mathrm{N} \backslash \mathrm{T}$ & 60 & 110 & 210 & 250 & $\mathrm{~N} \backslash \mathrm{T}$ & 60 & 110 & 210 & 250 \\
\hline 50 & 88.4 & 86.4 & 82.7 & 80.3 & 50 & 88.4 & 86.4 & 82.7 & 80.3 \\
\hline 100 & 94.1 & 92.3 & 90.7 & 88.9 & 100 & 94.1 & 92.3 & 90.7 & 88.9 \\
\hline 200 & 99.8 & 99.2 & 99.4 & 99.2 & 200 & 99.8 & 99.2 & 99.4 & 99.2 \\
\hline 500 & 100 & 100 & 100 & 100 & 500 & 100 & 100 & 100 & 100 \\
\hline \multicolumn{6}{|c|}{$k_{0}=2$} & \multicolumn{4}{|c|}{$k_{0}=2$} \\
\hline $\mathrm{N} \backslash \mathrm{T}$ & 60 & 110 & 210 & 250 & $\mathrm{~N} \backslash \mathrm{T}$ & 60 & 110 & 210 & 250 \\
\hline 50 & 61.6 & 55.9 & 47.7 & 44.3 & 50 & 61.6 & 55.9 & 47.7 & 44.3 \\
\hline 100 & 84.0 & 74.5 & 64.2 & 60.9 & 100 & 84.0 & 74.5 & 64.2 & 60.9 \\
\hline 200 & 98.6 & 97.7 & 94.2 & 94.1 & 200 & 98.6 & 97.7 & 94.2 & 94.1 \\
\hline 500 & 100 & 100 & 100 & 99.9 & 500 & 100 & 100 & 100 & 99.9 \\
\hline \multicolumn{10}{|c|}{$\begin{array}{l}\text { Notes: } \sigma^{2} \text { thresholding is implemented using Algorithm } 1 \text { in the main article, with } \\
p_{\max }=m_{0}+k_{0}+1 \text {, where } m_{0} \text { is the true number of dominant units (if any) and } \\
k_{0} \text { is the number of external factors. S }-\sigma^{2} \text { and SMT }-\sigma^{2} \text { refer to Sequential } \sigma^{2} \\
\text { thresholding and Sequential-MT } \sigma^{2} \text { thresholding, as implemented using Algorithms } \\
2 \text { and } 3 \text { in the main article, respectively. Threshold in the } \sigma^{2} \text { thresholding step of all } \\
\text { three algorithms is given by } \hat{\sigma}_{i T}^{2} \leq 2 \hat{\eta}_{i N}^{2} N^{-1} \log (T) \text {. The threshold chosen for } N>T \\
\text { in the alternative version of SMT }-\sigma^{2} \text { is given by } \hat{\sigma}_{i T}^{2} \leq 2 \hat{\sigma}_{u i}^{2} T^{-1} \log (T) \text {. See Section } \\
\text { A . } 3 \text { for further details }\end{array}$} \\
\hline
\end{tabular}


Table S3.2: Average number of non-dominant units falsely selected as dominant $\left(m_{0}=0\right)$

\begin{tabular}{|c|c|c|c|c|c|c|c|c|c|}
\hline \multicolumn{6}{|c|}{$\begin{array}{c}\sigma^{2} \text { thresholding } \\
k_{0}=0\end{array}$} & \multicolumn{4}{|c|}{$\begin{array}{l}\mathrm{S}-\sigma^{2} \\
k_{0}=0\end{array}$} \\
\hline $\mathrm{N} \backslash \mathrm{T}$ & 60 & 110 & 210 & 250 & $\mathrm{~N} \backslash \mathrm{T}$ & 60 & 110 & 210 & 250 \\
\hline 50 & 0 & 0.1 & 0.1 & 0.1 & 50 & 0 & 0.1 & 0.1 & 0.1 \\
\hline 100 & 0 & 0 & 0 & 0 & 100 & 0 & 0 & 0 & 0 \\
\hline 200 & 0 & 0 & 0 & 0 & 200 & 0 & 0 & 0 & 0 \\
\hline 500 & 0 & 0 & 0 & 0 & 500 & 0 & 0 & 0 & 0 \\
\hline \multicolumn{6}{|c|}{$k_{0}=1$} & \multicolumn{4}{|c|}{$k_{0}=1$} \\
\hline $\mathrm{N} \backslash \mathrm{T}$ & 60 & 110 & 210 & 250 & $\mathrm{~N} \backslash \mathrm{T}$ & 60 & 110 & 210 & 250 \\
\hline 50 & 0.4 & 0.6 & 0.7 & 0.7 & 50 & 0.5 & 0.7 & 0.8 & 0.8 \\
\hline 100 & 0.1 & 0.2 & 0.3 & 0.3 & 100 & 0.1 & 0.2 & 0.3 & 0.4 \\
\hline 200 & 0 & 0 & 0 & 0 & 200 & 0 & 0 & 0 & 0 \\
\hline 500 & 0 & 0 & 0 & 0 & 500 & 0 & 0 & 0 & 0 \\
\hline \multicolumn{6}{|c|}{$k_{0}=2$} & \multicolumn{4}{|c|}{$k_{0}=2$} \\
\hline $\mathrm{N} \backslash \mathrm{T}$ & 60 & 110 & 210 & 250 & $\mathrm{~N} \backslash \mathrm{T}$ & 60 & 110 & 210 & 250 \\
\hline 50 & 0.9 & 1.1 & 1.4 & 1.4 & 50 & 0.9 & 1.2 & 1.5 & 1.5 \\
\hline 100 & 0.2 & 0.5 & 0.7 & 0.8 & 100 & 0.3 & 0.5 & 0.8 & 0.9 \\
\hline 200 & 0 & 0 & 0.1 & 0.1 & 200 & 0 & 0 & 0.1 & 0.1 \\
\hline 500 & 0 & 0 & 0 & 0 & 500 & 0 & 0 & 0 & 0 \\
\hline \multicolumn{5}{|c|}{$\begin{array}{c}\mathrm{SMT}-\sigma^{2} \\
k_{0}=0\end{array}$} & \multicolumn{5}{|c|}{$\begin{array}{c}\mathrm{SMT}-\sigma^{2}, \text { alternative scaling } \\
k_{0}=0\end{array}$} \\
\hline $\mathrm{N} \backslash \mathrm{T}$ & 60 & 110 & 210 & 250 & $\mathrm{~N} \backslash \mathrm{T}$ & 60 & 110 & 210 & 250 \\
\hline 50 & 0 & 0 & 0 & 0 & 50 & 0 & 0 & 0 & 0 \\
\hline 100 & 0 & 0 & 0 & 0 & 100 & 0 & 0 & 0 & 0 \\
\hline 200 & 0 & 0 & 0 & 0 & 200 & 0 & 0 & 0 & 0 \\
\hline 500 & 0 & 0 & 0 & 0 & 500 & 0 & 0 & 0 & 0 \\
\hline \multicolumn{6}{|c|}{$k_{0}=1$} & \multicolumn{4}{|c|}{$k_{0}=1$} \\
\hline $\mathrm{N} \backslash \mathrm{T}$ & 60 & 110 & 210 & 250 & $\mathrm{~N} \backslash \mathrm{T}$ & 60 & 110 & 210 & 250 \\
\hline 50 & 0.1 & 0.2 & 0.2 & 0.2 & 50 & 0.1 & 0.2 & 0.2 & 0.2 \\
\hline 100 & 0.1 & 0.1 & 0.1 & 0.1 & 100 & 0.1 & 0.1 & 0.1 & 0.1 \\
\hline 200 & 0 & 0 & 0 & 0 & 200 & 0 & 0 & 0 & 0 \\
\hline 500 & 0 & 0 & 0 & 0 & 500 & 0 & 0 & 0 & 0 \\
\hline \multicolumn{6}{|c|}{$k_{0}=2$} & \multicolumn{4}{|c|}{$k_{0}=2$} \\
\hline $\mathrm{N} \backslash \mathrm{T}$ & 60 & 110 & 210 & 250 & $\mathrm{~N} \backslash \mathrm{T}$ & 60 & 110 & 210 & 250 \\
\hline 50 & 0.4 & 0.5 & 0.6 & 0.7 & 50 & 0.4 & 0.5 & 0.6 & 0.7 \\
\hline 100 & 0.2 & 0.3 & 0.4 & 0.4 & 100 & 0.2 & 0.3 & 0.4 & 0.4 \\
\hline 200 & 0 & 0 & 0.1 & 0.1 & 200 & 0 & 0 & 0.1 & 0.1 \\
\hline 500 & 0 & 0 & 0 & 0 & 500 & 0 & 0 & 0 & 0 \\
\hline
\end{tabular}

Notes: See the notes to Table S3.1. 


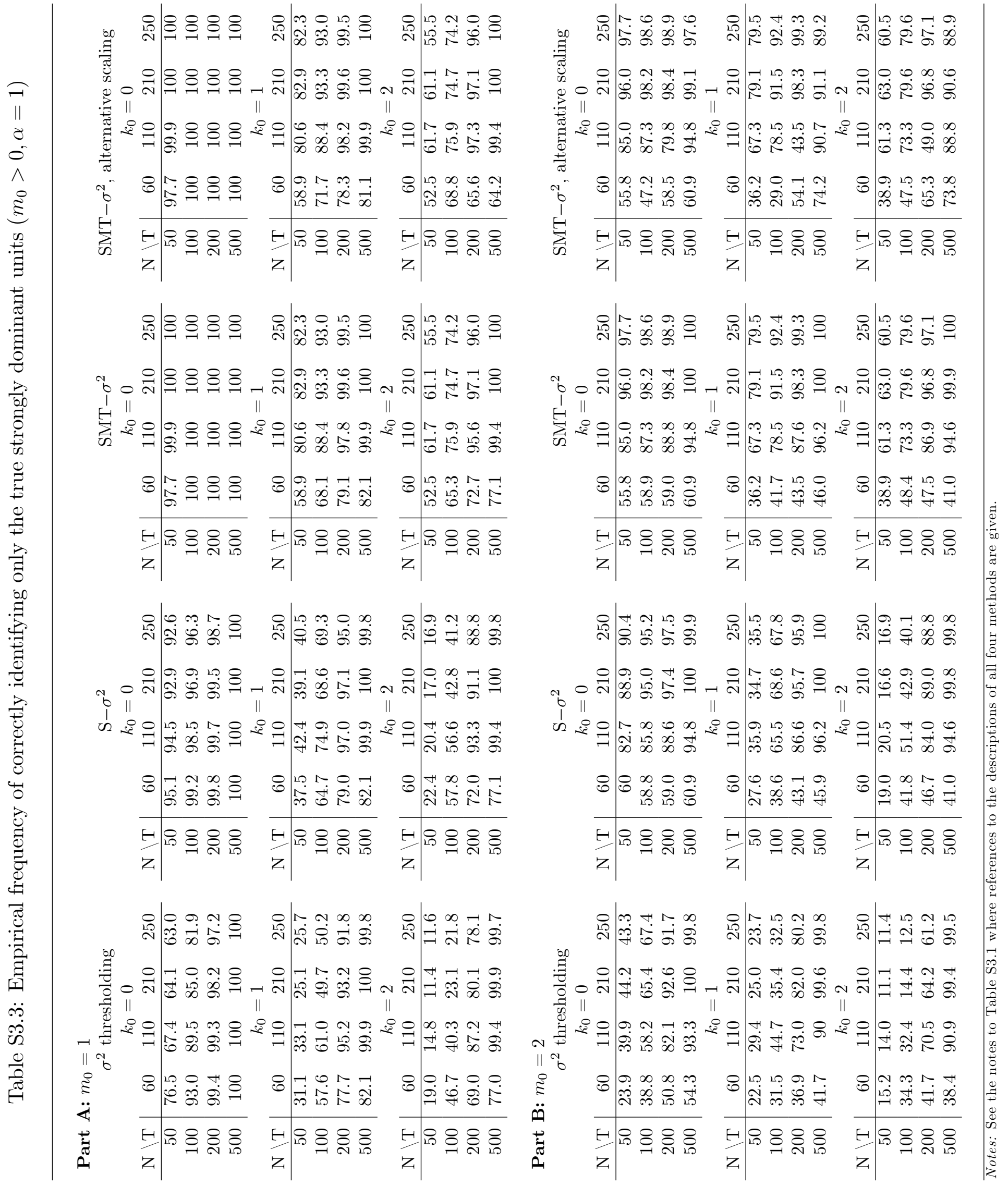



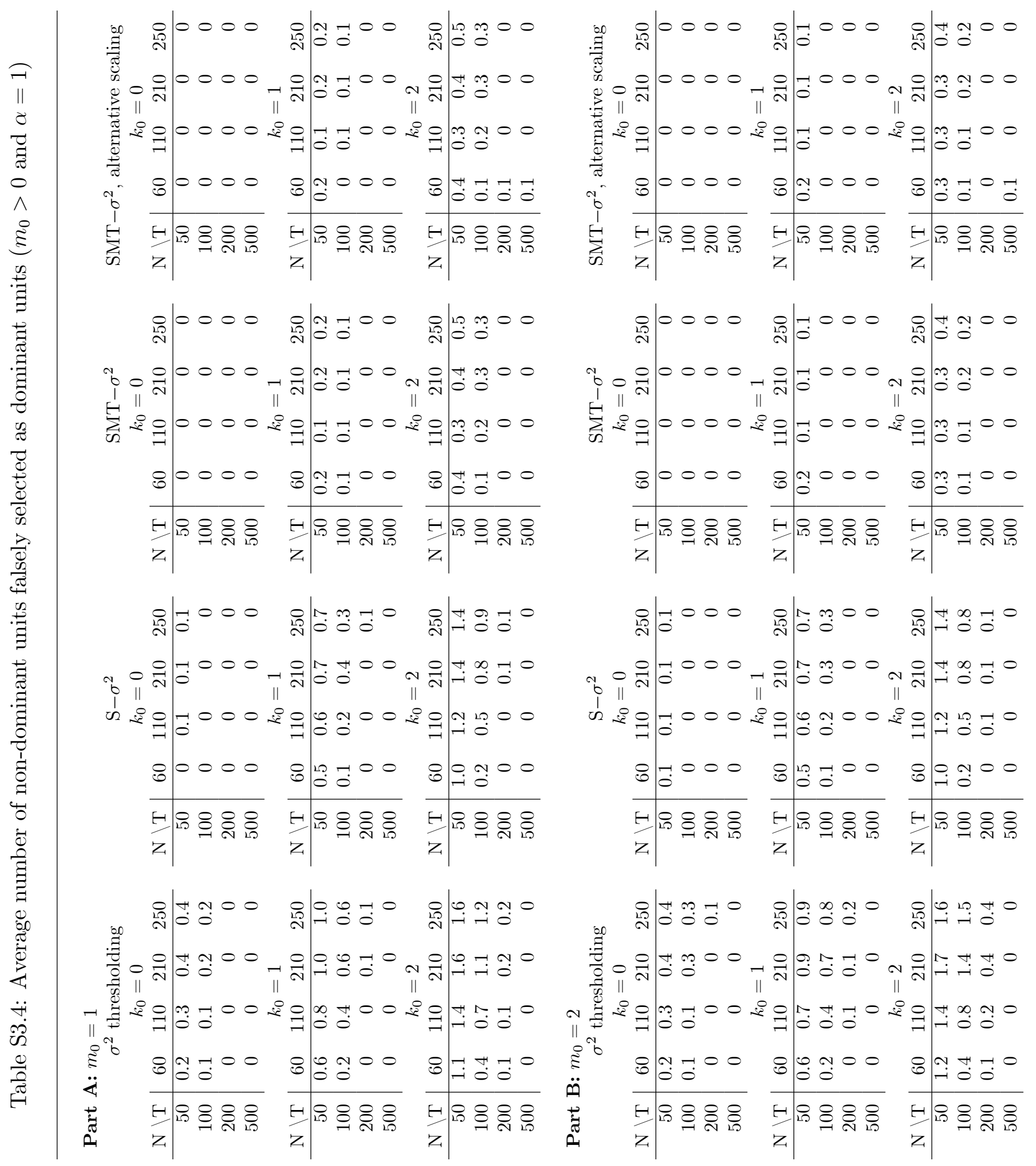

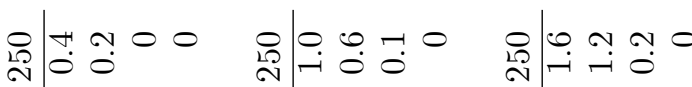

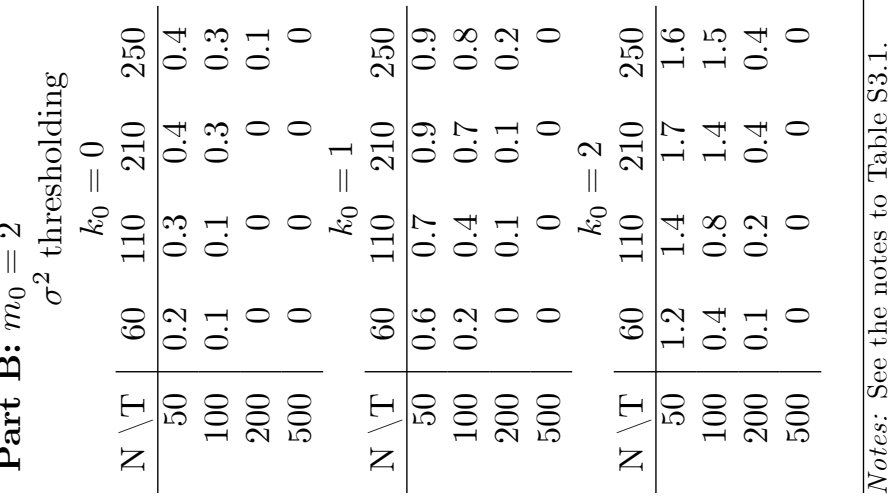




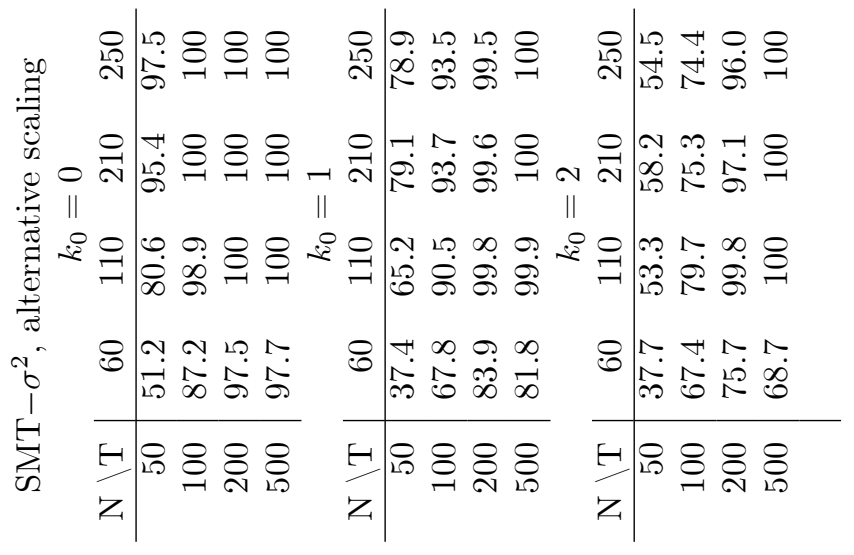

络|

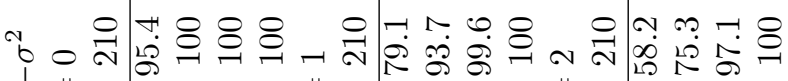

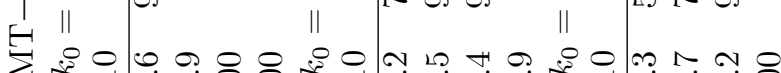
苔

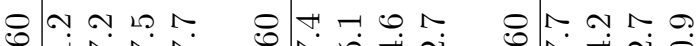

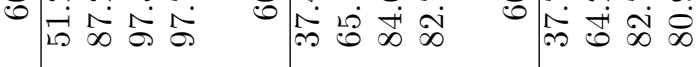

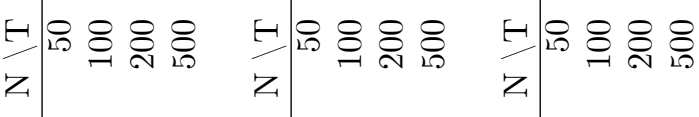

虫| • O u

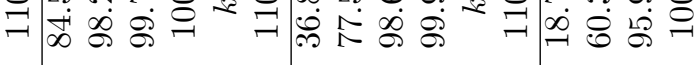
8 政r

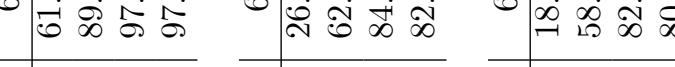

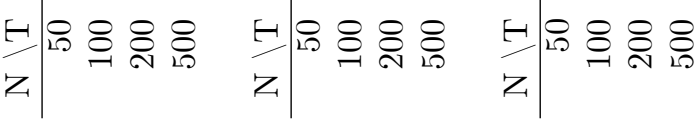

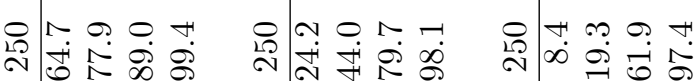
承

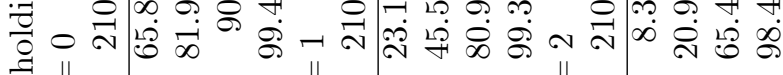
फु

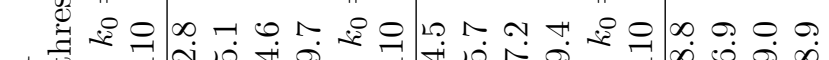

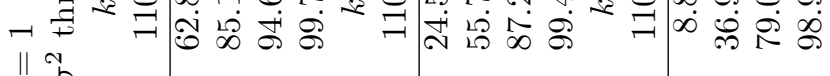

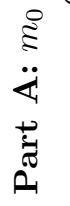

$$
\frac{8}{\mathrm{~B}}
$$

ㅂ.

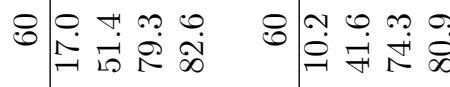

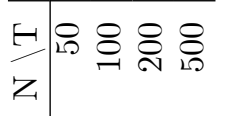

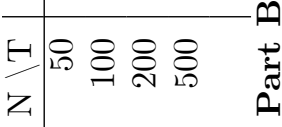

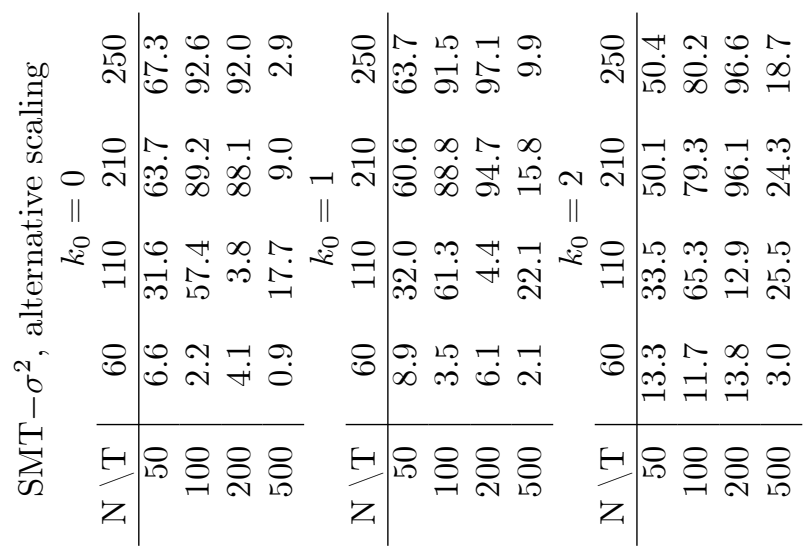

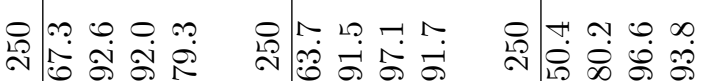
。

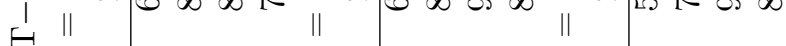

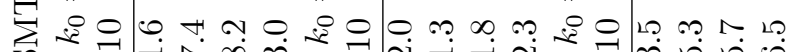

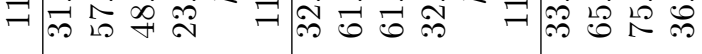

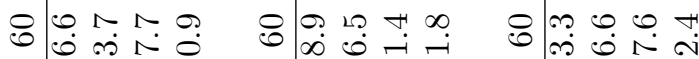
苝|

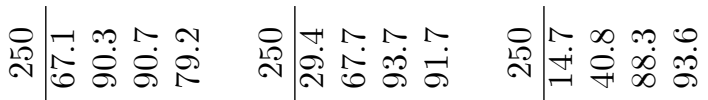

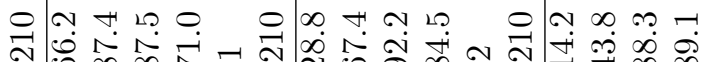

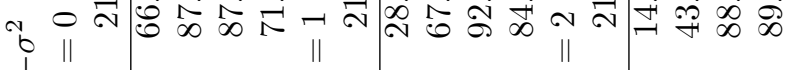
我

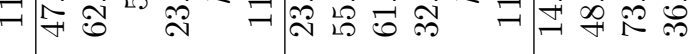

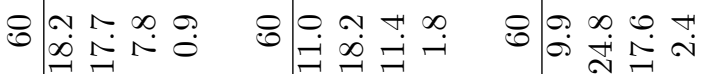

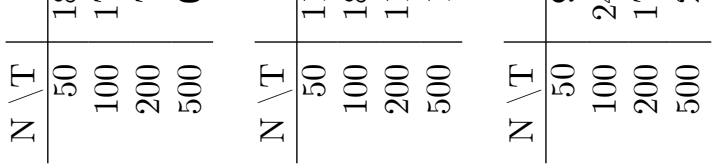

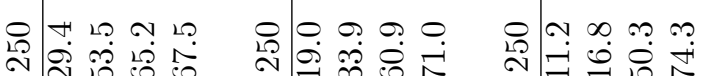

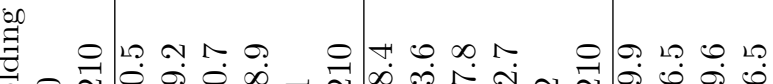
O 皮 ค च

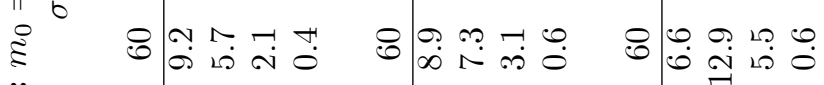

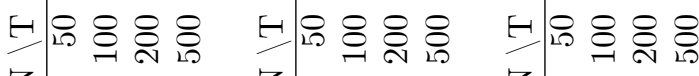

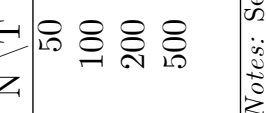




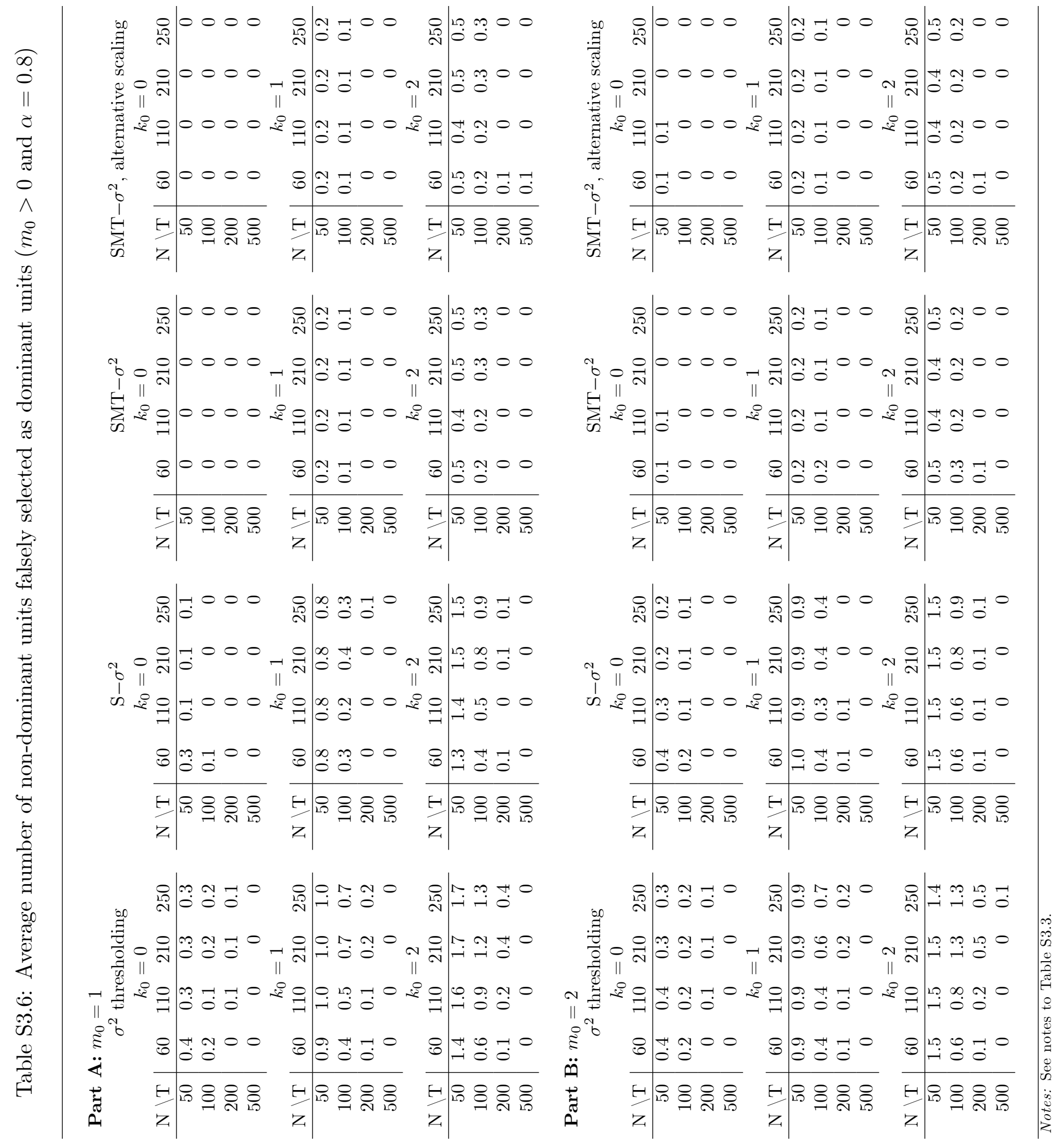




\section{S4 Maximum difference and maximum ratio threshold- ing}

The idea of considering the maximum difference or the maximum ratio between two ordered statistics, has been recently suggested by Ahn and Horenstein (2013) and used by Brownlees and Mesters (2018) in the context of detecting dominant units, can also be applied to $\sigma^{2}$ thresholding. Denote by $\hat{\sigma}_{(1) T}^{2}, \hat{\sigma}_{(2) T}^{2}, \ldots, \hat{\sigma}_{(N) T}^{2}$ the ordered estimated error variances in ascending order for a dataset with $N$ cross section units and $T$ time periods. Then the following two simple algorithms can be considered:

\section{Algorithm 6 (Max $\sigma^{2}$-difference algorithm)}

1. Conduct $\sigma^{2}$ thresholding using $p_{\max }$ estimated factors. If the estimated number of dominant units, denoted by $\widetilde{m}$, is zero, stop and conclude that there is no dominant unit. Otherwise, proceed with step 2.

2. Let the estimated number of dominant units be given by

$$
\hat{m}=\underset{j=1,2, \ldots, p_{\max }}{\arg \max }\left(\hat{\sigma}_{(j+1) T}^{2}-\hat{\sigma}_{(j) T}^{2}\right)
$$

and the estimated identities by the indices of the units whose estimated error variances are $\hat{\sigma}_{(1) T}^{2}, \hat{\sigma}_{(2) T}^{2}, \ldots, \hat{\sigma}_{(\hat{m}) T}^{2}$.

\section{Algorithm 7 (Max $\sigma^{2}$-ratio algorithm)}

1. Conduct $\sigma^{2}$ thresholding using $p_{\max }$ estimated factors. If $\widetilde{m}=0$, stop and conclude that there is no dominant unit. Otherwise, proceed to step 2.

2. Let the estimated number of dominant units be given by

$$
\hat{m}=\underset{j=1, \ldots, p_{\max }}{\arg \max }\left(\frac{\hat{\sigma}_{(j+1) T}^{2}}{\hat{\sigma}_{(j) T}^{2}}\right),
$$

and the estimated identities by the indices of the units whose estimated error variances are $\hat{\sigma}_{(1) T}^{2}, \hat{\sigma}_{(2) T}^{2}, \ldots, \hat{\sigma}_{(\hat{m}) T}^{2}$.

In Table S4.1 we report the performance of the two approaches described above using the Monte Carlo set up described in Section 6 of the paper. The case $m=0$ is left out since the probability of correctly detecting the absence of dominant units is entirely determined by the initial $\sigma^{2}$ thresholding step of max difference and max ratio thresholding methods. Results for models with at least one dominant unit show that the two algorithms, based on either the maximum difference or the maximum ratio, perform quite similarly to the SMT $-\sigma^{2}$ thresholding. However, the former two methods exhibit inferior performance in samples where $N$ is small. This comparative disadvantage is compensated by a superior performance in cases where there are both external common factors and more than one dominant units. However, empirical evidence for the presence of at least one dominant unit in the existing applied literature is rather 
limited. ${ }^{21}$ Furthermore, the relative advantage of max difference or max ratio thresholding disappears when weakly dominant units are considered. As reported in Table S4.3, SMT $-\sigma^{2}$ thresholding has a performance comparable to that of the two new algorithms considered here, even when $N$ is large and the number of dominant units is larger than 1.

Tables S4.2 and S4.4 report the average numbers of falsely selected dominant units, and show that the max difference and max ratio thresholding procedures perform reasonably well. But as compared to SMT $-\sigma^{2}$ thresholding, the max thresholding approaches tend to show a higher proportion of false discoveries, and overall we are led to favor SMT $-\sigma^{2}$ thresholding over the max difference and the max ratio thresholding.

\footnotetext{
${ }^{21}$ see e.g. Pesaran and Yang (2016) or Dungey and Volkov (2018) who find that the degree of dominance of the most influential unit in their datasets is quite far from the value of 1 that would indicate a dominant unit in the sense of a factor common to all cross-section units.
} 
Table S4.1: Empirical frequency of correctly identifying only the true strongly dominant units $(m>0, \alpha=1)$

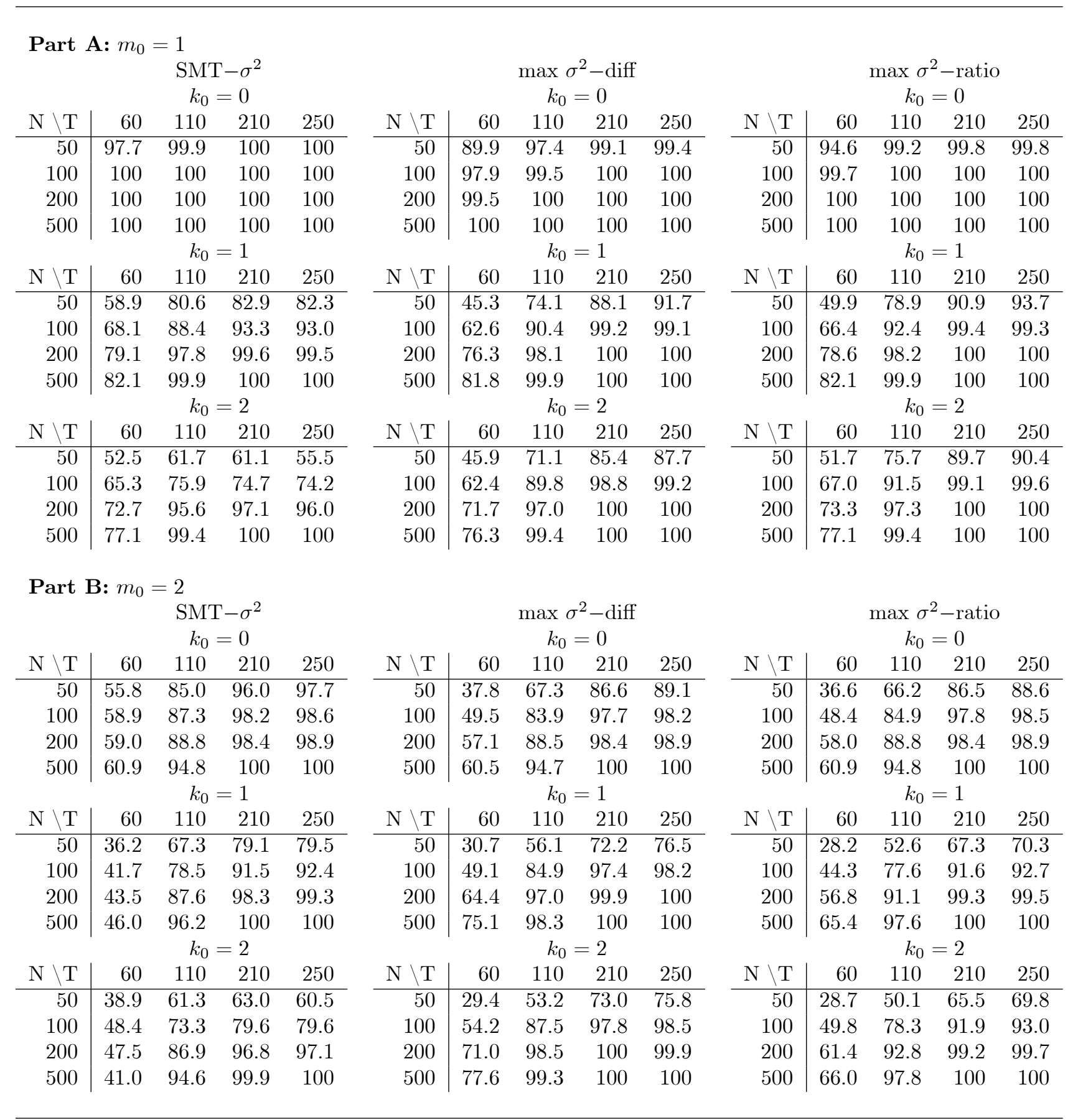

Notes: SMT $-\sigma^{2}$ refers to SMT $-\sigma^{2}$ thresholding, implemented with pmax $=m_{0}+k_{0}+1$ as described in Algorithm 5 . max $\sigma^{2}-\operatorname{diff}$ and $\max \sigma^{2}$-ratio denote detection of dominant units via algorithms 6 and 7 , conducted with $\operatorname{pmax}=m_{0}+k_{0}+1$. 
Table S4.2: Average number of non-dominant units falsely selected as dominant units $\left(m_{0}>0\right.$ and $\alpha=1$ )

\begin{tabular}{|c|c|c|c|c|c|c|c|c|c|c|c|c|c|c|}
\hline \multicolumn{15}{|c|}{ Part A: $m_{0}=1$} \\
\hline \multirow[b]{2}{*}{$\mathrm{N} \backslash \mathrm{T}$} & \multicolumn{4}{|c|}{$\begin{array}{c}\mathrm{SMT}-\sigma^{2} \\
k_{0}=0\end{array}$} & \multicolumn{6}{|c|}{$\begin{array}{c}\max \sigma^{2}-\operatorname{diff} \\
k_{0}=0\end{array}$} & \multicolumn{4}{|c|}{$\begin{array}{c}\max \sigma^{2} \text {-ratio } \\
k_{0}=0\end{array}$} \\
\hline & 60 & 110 & 210 & 250 & $\mathrm{~N} \backslash \mathrm{T}$ & 60 & 110 & 210 & 250 & $\mathrm{~N} \backslash \mathrm{T}$ & 60 & 110 & 210 & 250 \\
\hline 50 & 0 & 0 & 0 & 0 & 50 & 0.1 & 0 & 0 & 0 & 50 & 0.1 & 0 & 0 & 0 \\
\hline 100 & 0 & 0 & 0 & 0 & 100 & 0 & 0 & 0 & 0 & 100 & 0 & 0 & 0 & 0 \\
\hline 200 & 0 & 0 & 0 & 0 & 200 & 0 & 0 & 0 & 0 & 200 & 0 & 0 & 0 & 0 \\
\hline 500 & 0 & 0 & 0 & 0 & 500 & 0 & 0 & 0 & 0 & 500 & 0 & 0 & 0 & 0 \\
\hline & \multicolumn{4}{|c|}{$k_{0}=1$} & \multicolumn{6}{|c|}{$k_{0}=1$} & \multicolumn{4}{|c|}{$k_{0}=1$} \\
\hline $\mathrm{N} \backslash \mathrm{T}$ & 60 & 110 & 210 & 250 & $\mathrm{~N} \backslash \mathrm{T}$ & 60 & 110 & 210 & 250 & $\mathrm{~N} \backslash \mathrm{T}$ & 60 & 110 & 210 & 250 \\
\hline 50 & 0.2 & 0.1 & 0.2 & 0.2 & 50 & 0.5 & 0.2 & 0.1 & 0.1 & 50 & 0.4 & 0.2 & 0.1 & 0 \\
\hline 100 & 0.1 & 0.1 & 0.1 & 0.1 & 100 & 0.1 & 0 & 0 & 0 & 100 & 0.1 & 0 & 0 & 0 \\
\hline 200 & 0 & 0 & 0 & 0 & 200 & 0 & 0 & 0 & 0 & 200 & 0 & 0 & 0 & 0 \\
\hline 500 & 0 & 0 & 0 & 0 & 500 & 0 & 0 & 0 & 0 & 500 & 0 & 0 & 0 & 0 \\
\hline & \multicolumn{4}{|c|}{$k_{0}=2$} & \multicolumn{6}{|c|}{$k_{0}=2$} & \multicolumn{4}{|c|}{$k_{0}=2$} \\
\hline $\mathrm{N} \backslash \mathrm{T}$ & 60 & 110 & 210 & 250 & $\mathrm{~N} \backslash \mathrm{T}$ & 60 & 110 & 210 & 250 & $\mathrm{~N} \backslash \mathrm{T}$ & 60 & 110 & 210 & 250 \\
\hline 50 & 0.4 & 0.3 & 0.4 & 0.5 & 50 & 0.7 & 0.4 & 0.2 & 0.1 & 50 & 0.6 & 0.3 & 0.1 & 0.1 \\
\hline 100 & 0.1 & 0.2 & 0.3 & 0.3 & 100 & 0.2 & 0.1 & 0 & 0 & 100 & 0.2 & 0 & 0 & 0 \\
\hline 200 & 0 & 0 & 0 & 0 & 200 & 0 & 0 & 0 & 0 & 200 & 0 & 0 & 0 & 0 \\
\hline 500 & 0 & 0 & 0 & 0 & 500 & 0 & 0 & 0 & 0 & 500 & 0 & 0 & 0 & 0 \\
\hline \multicolumn{15}{|c|}{ Part B: $m_{0}=2$} \\
\hline & \multirow{2}{*}{\multicolumn{4}{|c|}{$\begin{array}{c}\mathrm{SMT}-\sigma^{2} \\
k_{0}=0\end{array}$}} & \multirow{2}{*}{\multicolumn{6}{|c|}{$\begin{array}{c}\max \sigma^{2}-\operatorname{diff} \\
\quad k_{0}=0\end{array}$}} & \multirow{2}{*}{\multicolumn{4}{|c|}{$\begin{array}{c}\max \sigma^{2} \text {-ratio } \\
k_{0}=0\end{array}$}} \\
\hline & & & & & & & & & & & & & & \\
\hline $\mathrm{N} \backslash \mathrm{T}$ & 60 & 110 & 210 & 250 & $\mathrm{~N} \backslash \mathrm{T}$ & 60 & 110 & 210 & 250 & $\mathrm{~N} \backslash \mathrm{T}$ & 60 & 110 & 210 & 250 \\
\hline 50 & 0 & 0 & 0 & 0 & 50 & 0.2 & 0.1 & 0.1 & 0.1 & 50 & 0.2 & 0.1 & 0 & 0 \\
\hline 100 & 0 & 0 & 0 & 0 & 100 & 0.1 & 0 & 0 & 0 & 100 & 0.1 & 0 & 0 & 0 \\
\hline 200 & 0 & 0 & 0 & 0 & 200 & 0 & 0 & 0 & 0 & 200 & 0 & 0 & 0 & 0 \\
\hline 500 & 0 & 0 & 0 & 0 & 500 & 0 & 0 & 0 & 0 & 500 & 0 & 0 & 0 & 0 \\
\hline & \multicolumn{4}{|c|}{$k_{0}=1$} & \multicolumn{6}{|c|}{$k_{0}=1$} & \multicolumn{4}{|c|}{$k_{0}=1$} \\
\hline $\mathrm{N} \backslash \mathrm{T}$ & 60 & 110 & 210 & 250 & $\mathrm{~N} \backslash \mathrm{T}$ & 60 & 110 & 210 & 250 & $\mathrm{~N} \backslash \mathrm{T}$ & 60 & 110 & 210 & 250 \\
\hline 50 & 0.2 & 0.1 & 0.1 & 0.1 & 50 & 0.4 & 0.2 & 0.1 & 0.1 & 50 & 0.3 & 0.1 & 0.1 & 0 \\
\hline 100 & 0 & 0 & 0 & 0 & 100 & 0.2 & 0.1 & 0 & 0 & 100 & 0.1 & 0 & 0 & 0 \\
\hline 200 & 0 & 0 & 0 & 0 & 200 & 0.1 & 0 & 0 & 0 & 200 & 0 & 0 & 0 & 0 \\
\hline 500 & 0 & 0 & 0 & 0 & 500 & 0 & 0 & 0 & 0 & 500 & 0 & 0 & 0 & 0 \\
\hline & \multicolumn{4}{|c|}{$k_{0}=2$} & \multicolumn{6}{|c|}{$k_{0}=2$} & \multicolumn{4}{|c|}{$k_{0}=2$} \\
\hline $\mathrm{N} \backslash \mathrm{T}$ & 60 & 110 & 210 & 250 & $\mathrm{~N} \backslash \mathrm{T}$ & 60 & 110 & 210 & 250 & $\mathrm{~N} \backslash \mathrm{T}$ & 60 & 110 & 210 & 250 \\
\hline 50 & 0.3 & 0.3 & 0.3 & 0.4 & 50 & 0.6 & 0.3 & 0.1 & 0.1 & 50 & 0.4 & 0.2 & 0.1 & 0.1 \\
\hline 100 & 0.1 & 0.1 & 0.2 & 0.2 & 100 & 0.2 & 0.1 & 0 & 0 & 100 & 0.1 & 0 & 0 & 0 \\
\hline 200 & 0 & 0 & 0 & 0 & 200 & 0.1 & 0 & 0 & 0 & 200 & 0 & 0 & 0 & 0 \\
\hline 500 & 0 & 0 & 0 & 0 & 500 & 0 & 0 & 0 & 0 & 500 & 0 & 0 & 0 & 0 \\
\hline
\end{tabular}

Notes: See the notes to Table S4.1. 
Table S4.3: Empirical frequency of correctly identifying only the true weakly dominant (influential) units $(m>0, \alpha=0.8)$

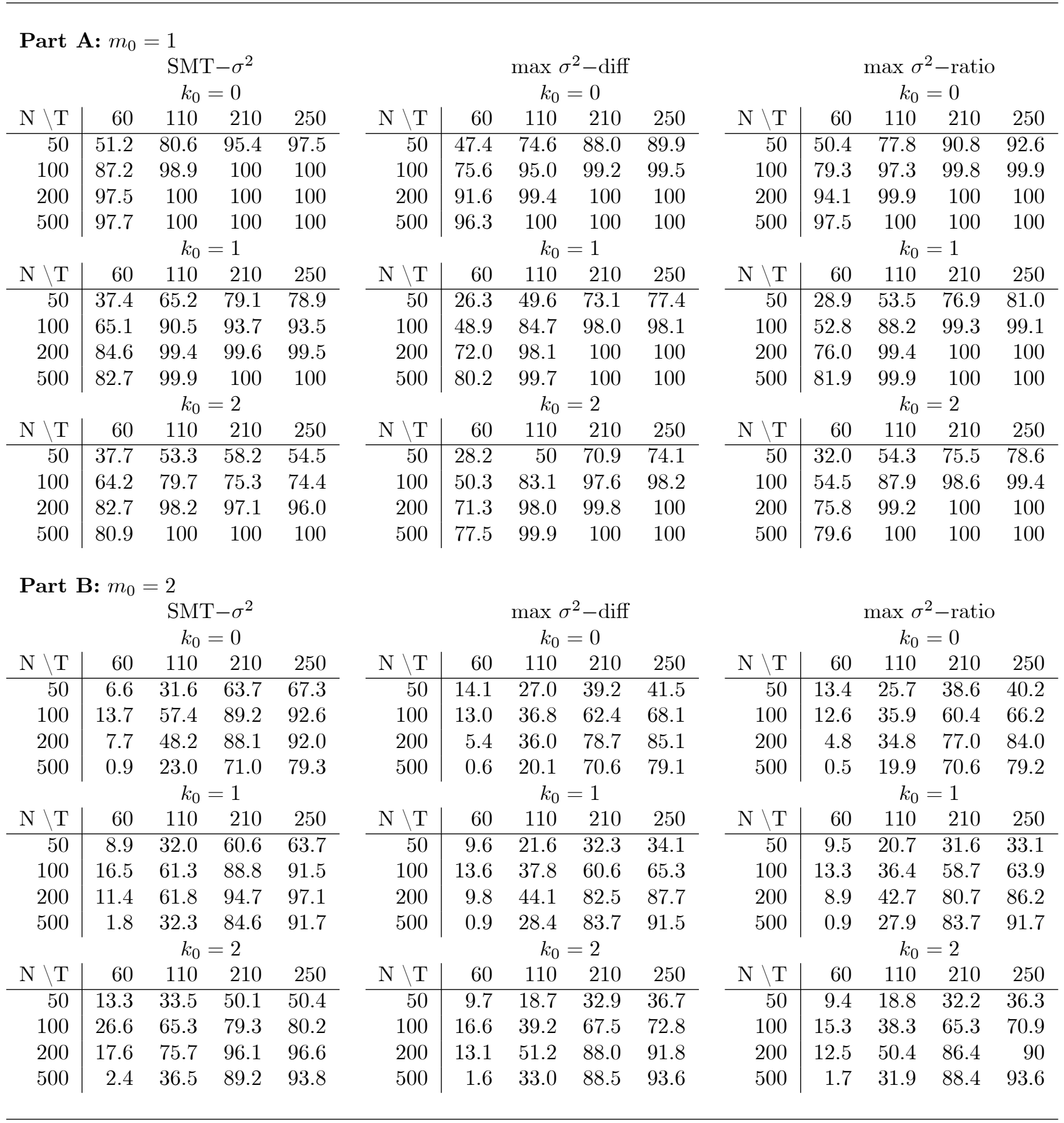

Notes: See the notes to Table S4.1. 
Table S4.4: Average number of non-dominant units falsely selected as dominant units $\left(m_{0}>0\right.$ and $\alpha=0.8$ )

Part A: $m_{0}=1$

\begin{tabular}{|c|c|c|c|c|c|c|c|c|c|c|c|c|c|c|}
\hline \multirow[b]{2}{*}{$\mathrm{N} \backslash \mathrm{T}$} & \multicolumn{4}{|c|}{$\begin{array}{c}\mathrm{SMT}-\sigma^{2} \\
k_{0}=0\end{array}$} & \multicolumn{6}{|c|}{$\begin{array}{c}\max \sigma^{2}-\operatorname{diff} \\
k_{0}=0\end{array}$} & \multicolumn{4}{|c|}{$\begin{array}{c}\max \sigma^{2} \text {-ratio } \\
k_{0}=0\end{array}$} \\
\hline & 60 & 110 & 210 & 250 & $\mathrm{~N} \backslash \mathrm{T}$ & 60 & 110 & 210 & 250 & $\mathrm{~N} \backslash \mathrm{T}$ & 60 & 110 & 210 & 250 \\
\hline 50 & 0 & 0 & 0 & 0 & 50 & 0.4 & 0.2 & 0.1 & 0.1 & 50 & 0.4 & 0.2 & 0.1 & 0.1 \\
\hline 100 & 0 & 0 & 0 & 0 & 100 & 0.2 & 0.1 & 0 & 0 & 100 & 0.2 & 0 & 0 & 0 \\
\hline 200 & 0 & 0 & 0 & 0 & 200 & 0.1 & 0 & 0 & 0 & 200 & 0 & 0 & 0 & 0 \\
\hline 500 & 0 & 0 & 0 & 0 & 500 & 0 & 0 & 0 & 0 & 500 & 0 & 0 & 0 & 0 \\
\hline & \multicolumn{4}{|c|}{$k_{0}=1$} & \multicolumn{6}{|c|}{$k_{0}=1$} & \multicolumn{4}{|c|}{$k_{0}=1$} \\
\hline $\mathrm{N} \backslash \mathrm{T}$ & 60 & 110 & 210 & 250 & $\mathrm{~N} \backslash \mathrm{T}$ & 60 & 110 & 210 & 250 & $\mathrm{~N} \backslash \mathrm{T}$ & 60 & 110 & 210 & 250 \\
\hline 50 & 0.2 & 0.2 & 0.2 & 0.2 & 50 & 0.9 & 0.7 & 0.3 & 0.3 & 50 & 0.8 & 0.6 & 0.3 & 0.2 \\
\hline 100 & 0.1 & 0.1 & 0.1 & 0.1 & 100 & 0.5 & 0.2 & 0 & 0 & 100 & 0.4 & 0.1 & 0 & 0 \\
\hline 200 & 0 & 0 & 0 & 0 & 200 & 0.2 & 0 & 0 & 0 & 200 & 0.1 & 0 & 0 & 0 \\
\hline 500 & 0 & 0 & 0 & 0 & 500 & 0 & 0 & 0 & 0 & 500 & 0 & 0 & 0 & 0 \\
\hline & \multicolumn{4}{|c|}{$k_{0}=2$} & \multicolumn{6}{|c|}{$k_{0}=2$} & \multicolumn{4}{|c|}{$k_{0}=2$} \\
\hline $\mathrm{N} \backslash \mathrm{T}$ & 60 & 110 & 210 & 250 & $\mathrm{~N} \backslash \mathrm{T}$ & 60 & 110 & 210 & 250 & $\mathrm{~N} \backslash \mathrm{T}$ & 60 & 110 & 210 & 250 \\
\hline 50 & 0.5 & 0.4 & 0.5 & 0.5 & 50 & 1.1 & 0.8 & 0.4 & 0.4 & 50 & 1.0 & 0.7 & 0.4 & 0.3 \\
\hline 100 & 0.2 & 0.2 & 0.3 & 0.3 & 100 & 0.6 & 0.2 & 0 & 0 & 100 & 0.5 & 0.2 & 0 & 0 \\
\hline 200 & 0 & 0 & 0 & 0 & 200 & 0.2 & 0 & 0 & 0 & 200 & 0.2 & 0 & 0 & 0 \\
\hline 500 & 0 & 0 & 0 & 0 & 500 & 0 & 0 & 0 & 0 & 500 & 0 & 0 & 0 & 0 \\
\hline
\end{tabular}

Part B: $m_{0}=2$

\begin{tabular}{|c|c|c|c|c|c|c|c|c|c|c|c|c|c|c|}
\hline \multirow[b]{2}{*}{$\mathrm{N} \backslash \mathrm{T}$} & \multicolumn{4}{|c|}{$\begin{array}{l}\mathrm{SMT}-\sigma^{2} \\
\quad k_{0}=0\end{array}$} & \multicolumn{6}{|c|}{$\begin{array}{c}\max \sigma^{2}-\operatorname{diff} \\
\quad k_{0}=0\end{array}$} & \multicolumn{4}{|c|}{$\begin{array}{c}\max \sigma^{2}-\text { ratio } \\
k_{0}=0\end{array}$} \\
\hline & 60 & 110 & 210 & 250 & $\mathrm{~N} \backslash \mathrm{T}$ & 60 & 110 & 210 & 250 & $\mathrm{~N} \backslash \mathrm{T}$ & 60 & 110 & 210 & 250 \\
\hline 50 & 0.1 & 0.1 & 0 & 0 & 50 & 0.5 & 0.4 & 0.3 & 0.3 & 50 & 0.5 & 0.4 & 0.2 & 0.2 \\
\hline 100 & 0 & 0 & 0 & 0 & 100 & 0.3 & 0.2 & 0.1 & 0.1 & 100 & 0.2 & 0.2 & 0.1 & 0.1 \\
\hline 200 & 0 & 0 & 0 & 0 & 200 & 0.1 & 0.1 & 0 & 0 & 200 & 0.1 & 0.1 & 0 & 0 \\
\hline 500 & 0 & 0 & 0 & 0 & 500 & 0 & 0 & 0 & 0 & 500 & 0 & 0 & 0 & 0 \\
\hline & \multicolumn{4}{|c|}{$k_{0}=1$} & \multicolumn{6}{|c|}{$k_{0}=1$} & \multicolumn{4}{|c|}{$k_{0}=1$} \\
\hline $\mathrm{N} \backslash \mathrm{T}$ & 60 & 110 & 210 & 250 & $\mathrm{~N} \backslash \mathrm{T}$ & 60 & 110 & 210 & 250 & $\mathrm{~N} \backslash \mathrm{T}$ & 60 & 110 & 210 & 250 \\
\hline 50 & 0.2 & 0.2 & 0.2 & 0.2 & 50 & 0.9 & 0.7 & 0.5 & 0.5 & 50 & 0.8 & 0.6 & 0.4 & 0.4 \\
\hline 100 & 0.2 & 0.1 & 0.1 & 0.1 & 100 & 0.6 & 0.4 & 0.2 & 0.2 & 100 & 0.5 & 0.4 & 0.2 & 0.2 \\
\hline 200 & 0 & 0 & 0 & 0 & 200 & 0.2 & 0.2 & 0.1 & 0 & 200 & 0.2 & 0.1 & 0 & 0 \\
\hline 500 & 0 & 0 & 0 & 0 & 500 & 0 & 0 & 0 & 0 & 500 & 0 & 0 & 0 & 0 \\
\hline \multicolumn{5}{|c|}{$k_{0}=2$} & \multicolumn{6}{|c|}{$k_{0}=2$} & \multicolumn{4}{|c|}{$k_{0}=2$} \\
\hline $\mathrm{N} \backslash \mathrm{T}$ & 60 & 110 & 210 & 250 & $\mathrm{~N} \backslash \mathrm{T}$ & 60 & 110 & 210 & 250 & $\mathrm{~N} \backslash \mathrm{T}$ & 60 & 110 & 210 & 250 \\
\hline 50 & 0.5 & 0.4 & 0.4 & 0.5 & 50 & 1.2 & 0.9 & 0.6 & 0.6 & 50 & 1.0 & 0.7 & 0.5 & 0.5 \\
\hline 100 & 0.3 & 0.2 & 0.2 & 0.2 & 100 & 0.8 & 0.5 & 0.2 & 0.2 & 100 & 0.7 & 0.4 & 0.1 & 0.1 \\
\hline 200 & 0.1 & 0 & 0 & 0 & 200 & 0.3 & 0.3 & 0.1 & 0 & 200 & 0.2 & 0.2 & 0 & 0 \\
\hline 500 & 0 & 0 & 0 & 0 & 500 & 0 & 0 & 0 & 0 & 500 & 0 & 0 & 0 & 0 \\
\hline
\end{tabular}




\section{S5 Simulation results for unmodified BM}

In the paper we have used a modified version of BM's detection method discussed in Section 6 of Brownlees and Mesters (2018), whereby only the $N / 2$ most connected cross-section units are considered when determining the number of dominant units. This section complements the simulations in Section 6 of the paper and report results for BM without this modification (henceforth unmodified BM). When implementing this procedure, the number of dominant units is determined from all $N$ cross section units in the dataset. All other details of the simulation exercise are as described in Section 6 of the paper.

Results on the probability of correctly determining the absence of dominant units from the data are left out since BM selects at least one unit as dominant by construction. The results for experiments with $m_{0}>0$ are summarized in Table S5.1. As can be seen the average number of units detected as dominant turns out to be much larger as compared to the modified BM. In fact, more than half of the cross section units in the sample are, on average, found to be dominant. In some cases, standardization of the data leads to a considerable decrease in the number of detected units. However, the set of cross section units falsely identified as dominant continues to be sizeable.

In cases where the data are driven by at least one dominant unit, unmodified BM method exhibits a reasonable performance if $T-N$ is large enough, and if the data is not standardized (see Table S5.2). By contrast, standardizing individual-specific time series has severe consequences for the probability of correctly detecting the true dominant units, especially in the presence of external factors. As can be seen from Table S5.4, the same results obtain if the true dominant units are weakly dominant. The average number of units falsely detected as dominant can be substantial. See Table S5.3 and Table S5.5). 
Table S5.1: Average number of non-dominant units falsely selected as dominant $\left(m_{0}=0\right)$

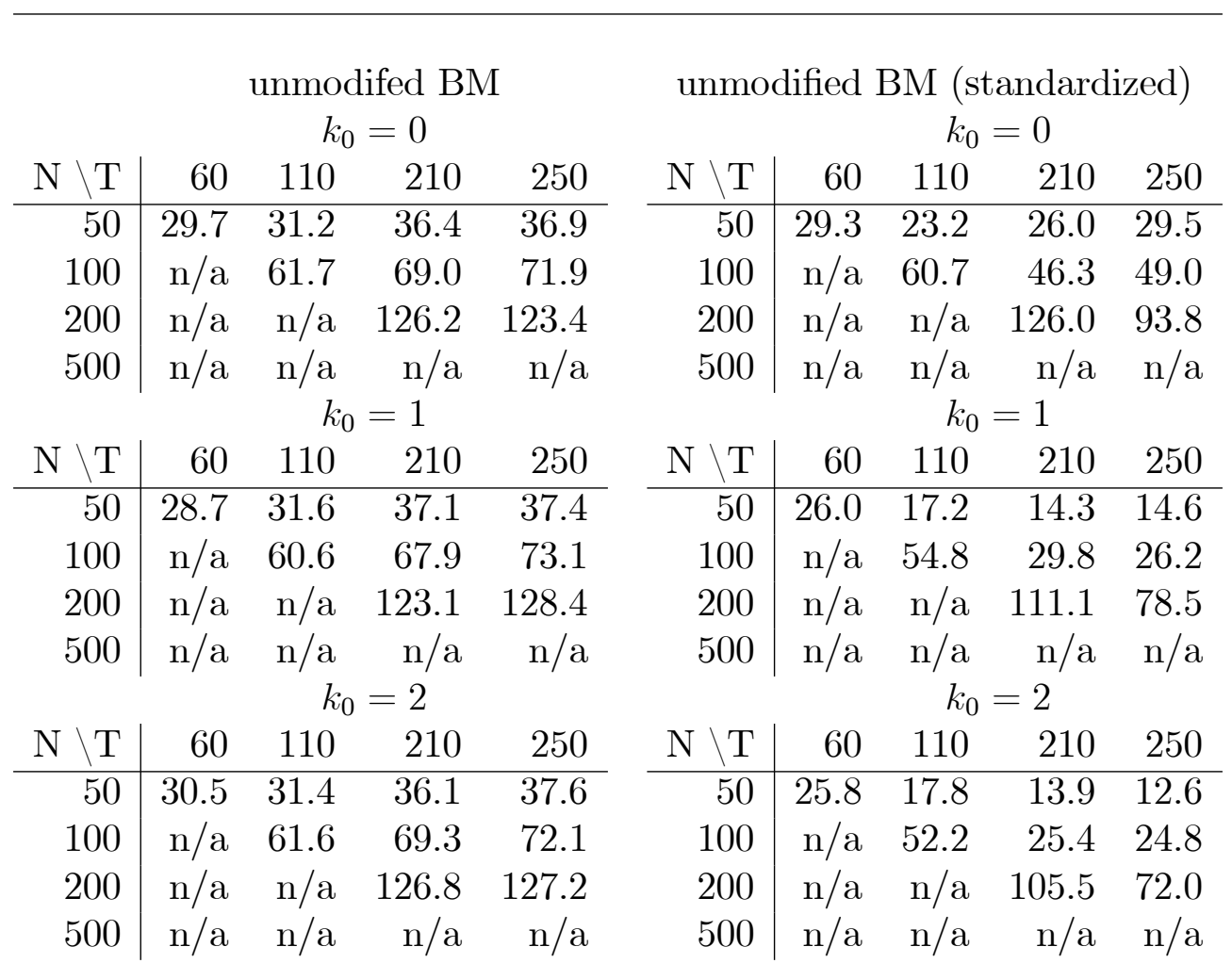

Notes: unmodified BM refers to the detection method of Brownlees and Mesters (2018) as introduced formally in Section 3 of their paper. unmodified BM (standardized) stands for application of unmodified BM to data that have been recentered and rescaled so that each cross-section specific time-series has an average of zero and a variance of one. BM methods are not applicable (n/a) if $T<N$. 
Table S5.2: Empirical frequency of correctly identifying only the true strongly dominant units $\left(m_{0}>0\right.$, and $\left.\alpha=1\right)$

Part A: $m_{0}=1$

unmodifed BM

$$
k_{0}=0
$$

\begin{tabular}{r|rrrr}
$\mathrm{N} \backslash \mathrm{T}$ & 60 & 110 & 210 & 250 \\
\hline 50 & 48.4 & 98.3 & 100.0 & 100.0 \\
100 & $\mathrm{n} / \mathrm{a}$ & 73.6 & 100.0 & 100.0 \\
200 & $\mathrm{n} / \mathrm{a}$ & $\mathrm{n} / \mathrm{a}$ & 89.6 & 100.0 \\
500 & $\mathrm{n} / \mathrm{a}$ & $\mathrm{n} / \mathrm{a}$ & $\mathrm{n} / \mathrm{a}$ & $\mathrm{n} / \mathrm{a}$
\end{tabular}

$$
k_{0}=1
$$

\begin{tabular}{r|rrrr}
$\mathrm{N} \backslash \mathrm{T}$ & 60 & 110 & 210 & 250 \\
\hline 50 & 42.1 & 96.7 & 99.9 & 99.9 \\
100 & $\mathrm{n} / \mathrm{a}$ & 67.3 & 100.0 & 100.0 \\
200 & $\mathrm{n} / \mathrm{a}$ & $\mathrm{n} / \mathrm{a}$ & 85.0 & 100.0 \\
500 & $\mathrm{n} / \mathrm{a}$ & $\mathrm{n} / \mathrm{a}$ & $\mathrm{n} / \mathrm{a}$ & $\mathrm{n} / \mathrm{a}$
\end{tabular}

$$
k_{0}=2
$$

\begin{tabular}{|c|c|c|c|c|c|c|c|c|c|}
\hline & & $\begin{array}{r}\mathrm{inmo} \\
k_{\mathrm{c}}\end{array}$ & $\begin{array}{l}\text { fed BI } \\
=0\end{array}$ & & unm & lified & BM & $\begin{array}{l}\text { indar } \\
=0\end{array}$ & \\
\hline $\mathrm{N} \backslash \mathrm{T}$ & 60 & 110 & 210 & 250 & $\mathrm{~N} \backslash \mathrm{T}$ & 60 & 110 & 210 & 250 \\
\hline 50 & 23.6 & 91.7 & 99.4 & 99.7 & 50 & 5.4 & 40.8 & 68.7 & 72.4 \\
\hline 100 & $\mathrm{n} / \mathrm{a}$ & 46.0 & 100.0 & 100.0 & 100 & $\mathrm{n} / \mathrm{a}$ & 16.7 & 94.9 & 98.4 \\
\hline 200 & $\mathrm{n} / \mathrm{a}$ & $\mathrm{n} / \mathrm{a}$ & 66.5 & 100.0 & 200 & $\mathrm{n} / \mathrm{a}$ & $\mathrm{n} / \mathrm{a}$ & 38.1 & 97.8 \\
\hline 500 & $\mathrm{n} / \mathrm{a}$ & $\mathrm{n} / \mathrm{a}$ & $\mathrm{n} / \mathrm{a}$ & $\mathrm{n} / \mathrm{a}$ & 500 & $\mathrm{n} / \mathrm{a}$ & $\mathrm{n} / \mathrm{a}$ & $\mathrm{n} / \mathrm{a}$ & $\mathrm{n} / \mathrm{a}$ \\
\hline & & & $=1$ & & & & & & \\
\hline $\mathrm{N} \backslash \mathrm{T}$ & 60 & 110 & 210 & 250 & $\mathrm{~N} \backslash \mathrm{T}$ & 60 & 110 & 210 & 250 \\
\hline 50 & 17.1 & 85.1 & 97.9 & 98.5 & 50 & 1.4 & 7.5 & 16.5 & 17.7 \\
\hline 100 & $\mathrm{n} / \mathrm{a}$ & 36.9 & 99.9 & 100.0 & 100 & $\mathrm{n} / \mathrm{a}$ & 5.6 & 58.5 & 63.1 \\
\hline 200 & $\mathrm{n} / \mathrm{a}$ & $\mathrm{n} / \mathrm{a}$ & 55.7 & 99.9 & 200 & $\mathrm{n} / \mathrm{a}$ & $\mathrm{n} / \mathrm{a}$ & 14.8 & 75.6 \\
\hline 500 & $\mathrm{n} / \mathrm{a}$ & $\mathrm{n} / \mathrm{a}$ & $\mathrm{n} / \mathrm{a}$ & $\mathrm{n} / \mathrm{a}$ & 500 & $\mathrm{n} / \mathrm{a}$ & $\mathrm{n} / \mathrm{a}$ & $\mathrm{n} / \mathrm{a}$ & $\mathrm{n} / \mathrm{a}$ \\
\hline & & & $=2$ & & & & & $=2$ & \\
\hline $\mathrm{N} \backslash \mathrm{T}$ & 60 & 110 & 210 & 250 & $\mathrm{~N} \backslash \mathrm{T}$ & 60 & 110 & 210 & 250 \\
\hline 50 & 15.55 & 79.5 & 96.3 & 97.35 & 50 & 0.2 & 1.0 & 2.2 & 1.6 \\
\hline 100 & $\mathrm{n} / \mathrm{a}$ & 33.1 & 99.95 & 99.85 & 100 & $\mathrm{n} / \mathrm{a}$ & 1.5 & 22.8 & 28.7 \\
\hline 200 & $\mathrm{n} / \mathrm{a}$ & $\mathrm{n} / \mathrm{a}$ & 50.35 & 99.65 & 200 & $\mathrm{n} / \mathrm{a}$ & $\mathrm{n} / \mathrm{a}$ & 5.8 & 46.0 \\
\hline 500 & $\mathrm{n} / \mathrm{a}$ & $\mathrm{n} / \mathrm{a}$ & $\mathrm{n} / \mathrm{a}$ & $\mathrm{n} / \mathrm{a}$ & 500 & $\mathrm{n} / \mathrm{a}$ & $\mathrm{n} / \mathrm{a}$ & $\mathrm{n} / \mathrm{a}$ & $\mathrm{n} / \mathrm{a}$ \\
\hline
\end{tabular}

\begin{tabular}{r|rrrr}
$\mathrm{N} \backslash \mathrm{T}$ & 60 & 110 & 210 & 250 \\
\hline 50 & 36.7 & 95.4 & 99.6 & 99.8 \\
100 & $\mathrm{n} / \mathrm{a}$ & 63.6 & 100 & 100 \\
200 & $\mathrm{n} / \mathrm{a}$ & $\mathrm{n} / \mathrm{a}$ & 83.7 & 100 \\
500 & $\mathrm{n} / \mathrm{a}$ & $\mathrm{n} / \mathrm{a}$ & $\mathrm{n} / \mathrm{a}$ & $\mathrm{n} / \mathrm{a}$
\end{tabular}

Part B: $m_{0}=2$ unmodified BM (standardized)

$$
k_{0}=0
$$

\begin{tabular}{r|rrrr}
$\mathrm{N} \backslash \mathrm{T}$ & 60 & 110 & 210 & 250 \\
\hline 50 & 47.0 & 98.2 & 99.7 & 99.9 \\
100 & n/a & 69.3 & 100.0 & 100.0 \\
200 & n/a & n/a & 87.4 & 100.0 \\
500 & n/a & n/a & n/a & n/a \\
& \multicolumn{4}{c}{$k_{0}=1$}
\end{tabular}

$100 \quad \mathrm{n} / \mathrm{a} \quad 47.6 \quad 99.0 \quad 99.8$

$200 \quad \mathrm{n} / \mathrm{a} \quad \mathrm{n} / \mathrm{a} \quad 69.4 \quad 99.9$

$500 \mathrm{n} / \mathrm{a} \quad \mathrm{n} / \mathrm{a} \quad \mathrm{n} / \mathrm{a} \quad \mathrm{n} / \mathrm{a}$

$$
k_{0}=2
$$

\begin{tabular}{r|rrrr}
$\mathrm{N} \backslash \mathrm{T}$ & 60 & 110 & 210 & 250 \\
\hline 50 & 12.8 & 45.1 & 62.7 & 65.4 \\
100 & $\mathrm{n} / \mathrm{a}$ & 29.6 & 94.4 & 96.0 \\
200 & $\mathrm{n} / \mathrm{a}$ & $\mathrm{n} / \mathrm{a}$ & 53.3 & 98.3 \\
500 & $\mathrm{n} / \mathrm{a}$ & $\mathrm{n} / \mathrm{a}$ & $\mathrm{n} / \mathrm{a}$ & $\mathrm{n} / \mathrm{a}$
\end{tabular}

unmodified BM (standardized)

\begin{tabular}{r|rrrr}
$\mathrm{N} \backslash \mathrm{T}$ & 60 & 110 & 210 & 250 \\
\hline 50 & 5.4 & 40.8 & 68.7 & 72.4
\end{tabular}

$\begin{array}{lllll}100 & \mathrm{n} / \mathrm{a} & 16.7 & 94.9 & 98.4\end{array}$

$200 \quad \mathrm{n} / \mathrm{a} \quad \mathrm{n} / \mathrm{a} \quad 38.1 \quad 97.8$

$k_{0}=1$

$\begin{array}{lllll}50 & 1.4 & 7.5 & 16.5 & 17.7\end{array}$

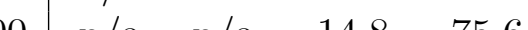

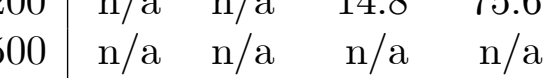$$
\begin{array}{llll}
n / a & n / a & n / a & n / a
\end{array}
$$

\begin{tabular}{r|rrrr}
$\mathrm{N} \backslash \mathrm{T}$ & 60 & 110 & 210 & 250 \\
\hline 50 & 25.6 & 74.7 & 90.7 & 93.1
\end{tabular}


Table S5.3: Average number of non-dominant units falsely selected as dominant $\left(m_{0}>0\right.$, and $\alpha=1$ )

Part A: $m_{0}=1$

unmodifed BM

\begin{tabular}{r|rrrr}
\multicolumn{5}{c}{$k_{0}=0$} \\
$\mathrm{~N} \backslash \mathrm{T}$ & 60 & 110 & 210 & 250 \\
\hline 50 & 14.7 & 0.3 & 0.0 & 0.0 \\
100 & $\mathrm{n} / \mathrm{a}$ & 16.0 & 0.0 & 0.0 \\
200 & $\mathrm{n} / \mathrm{a}$ & $\mathrm{n} / \mathrm{a}$ & 12.1 & 0.0 \\
500 & $\mathrm{n} / \mathrm{a}$ & $\mathrm{n} / \mathrm{a}$ & $\mathrm{n} / \mathrm{a}$ & $\mathrm{n} / \mathrm{a}$ \\
& & $k_{0}=1$
\end{tabular}

\begin{tabular}{r|rrrr}
$\mathrm{N} \backslash \mathrm{T}$ & 60 & 110 & 210 & 250 \\
\hline 50 & 17.6 & 0.6 & 0.0 & 0.0
\end{tabular}

$100 \mathrm{n} / \mathrm{a} \quad 19.6 \quad 0.0 \quad 0.0$

$200 \mathrm{n} / \mathrm{a} \quad \mathrm{n} / \mathrm{a} \quad 19.6 \quad 0.0$

$500 \mathrm{n} / \mathrm{a} \quad \mathrm{n} / \mathrm{a} \quad \mathrm{n} / \mathrm{a} \quad \mathrm{n} / \mathrm{a}$

$$
k_{0}=2
$$

\begin{tabular}{r|rrrr}
$\mathrm{N} \backslash \mathrm{T}$ & 60 & 110 & 210 & 250 \\
\hline 50 & 18.5 & 1.1 & 0.1 & 0.1 \\
100 & n/a & 21.6 & 0.0 & 0.0 \\
200 & n/a & n/a & 19.4 & 0.0 \\
500 & n/a & n/a & n/a & n/a
\end{tabular}

Part B: $m_{0}=2$

\begin{tabular}{|c|c|c|c|c|c|c|c|c|c|}
\hline \multicolumn{5}{|c|}{$\begin{array}{c}\text { unmodifed BM } \\
k_{0}=0\end{array}$} & \multicolumn{5}{|c|}{$\begin{array}{c}\text { unmodified BM (standardized) } \\
\qquad k_{0}=0\end{array}$} \\
\hline $\mathrm{N} \backslash \mathrm{T}$ & 60 & 110 & 210 & 250 & $\mathrm{~N} \backslash \mathrm{T}$ & 60 & 110 & 210 & 250 \\
\hline 50 & 11.7 & 0.4 & 0.0 & 0.0 & 50 & 16.9 & 2.2 & 0.4 & 0.5 \\
\hline 100 & $\mathrm{n} / \mathrm{a}$ & 8.2 & 0.0 & 0.0 & 100 & $\mathrm{n} / \mathrm{a}$ & 19.6 & 0.0 & 0.0 \\
\hline 200 & $\mathrm{n} / \mathrm{a}$ & $\mathrm{n} / \mathrm{a}$ & 6.2 & 0.0 & 200 & $\mathrm{n} / \mathrm{a}$ & $\mathrm{n} / \mathrm{a}$ & 18.2 & 0.0 \\
\hline 500 & $\mathrm{n} / \mathrm{a}$ & $\begin{array}{r}\mathrm{n} / \mathrm{a} \\
k_{0}\end{array}$ & $\begin{array}{l}\mathrm{n} / \mathrm{a} \\
1\end{array}$ & $\mathrm{n} / \mathrm{a}$ & 500 & $\mathrm{n} / \mathrm{a}$ & $\begin{array}{r}\mathrm{n} / \mathrm{a} \\
k_{0}\end{array}$ & $\begin{aligned} & \mathrm{n} / \mathrm{a} \\
= & 1\end{aligned}$ & $\mathrm{n} / \mathrm{a}$ \\
\hline $\mathrm{N} \backslash \mathrm{T}$ & 60 & 110 & 210 & 250 & $\mathrm{~N} \backslash \mathrm{T}$ & 60 & 110 & 210 & 250 \\
\hline 50 & 13.0 & 1.1 & 0.1 & 0.0 & 50 & 21.4 & 10.0 & 6.8 & 6.3 \\
\hline 100 & $\mathrm{n} / \mathrm{a}$ & 13.7 & 0.0 & 0.0 & 100 & $\mathrm{n} / \mathrm{a}$ & 33.7 & 1.0 & 0.7 \\
\hline 200 & $\mathrm{n} / \mathrm{a}$ & $\mathrm{n} / \mathrm{a}$ & 10.0 & 0.0 & 200 & $\mathrm{n} / \mathrm{a}$ & $\mathrm{n} / \mathrm{a}$ & 42.5 & 0.6 \\
\hline 500 & $\mathrm{n} / \mathrm{a}$ & $\begin{array}{r}\mathrm{n} / \mathrm{a} \\
k_{0}\end{array}$ & $\begin{aligned} & \mathrm{n} / \mathrm{a} \\
= & 2\end{aligned}$ & $\mathrm{n} / \mathrm{a}$ & 500 & $\mathrm{n} / \mathrm{a}$ & $\begin{array}{r}\mathrm{n} / \mathrm{a} \\
k_{0}\end{array}$ & $\begin{aligned} & \mathrm{n} / \mathrm{a} \\
= & 2\end{aligned}$ & $\mathrm{n} / \mathrm{a}$ \\
\hline $\mathrm{N} \backslash \mathrm{T}$ & 60 & 110 & 210 & 250 & $\mathrm{~N} \backslash \mathrm{T}$ & 60 & 110 & 210 & 250 \\
\hline 50 & 13.8 & 1.1 & 0.2 & 0.2 & 50 & 26.0 & 19.2 & 17.8 & 18.2 \\
\hline 100 & $\mathrm{n} / \mathrm{a}$ & 13.7 & 0.0 & 0.0 & 100 & $\mathrm{n} / \mathrm{a}$ & 44.0 & 8.3 & 6.3 \\
\hline 200 & $\mathrm{n} / \mathrm{a}$ & $\mathrm{n} / \mathrm{a}$ & 12.7 & 0.0 & 200 & $\mathrm{n} / \mathrm{a}$ & $\mathrm{n} / \mathrm{a}$ & 67.3 & 4.9 \\
\hline 500 & $\mathrm{n} / \mathrm{a}$ & $\mathrm{n} / \mathrm{a}$ & $\mathrm{n} / \mathrm{a}$ & $\mathrm{n} / \mathrm{a}$ & 500 & $\mathrm{n} / \mathrm{a}$ & $\mathrm{n} / \mathrm{a}$ & $\mathrm{n} / \mathrm{a}$ & $\mathrm{n} / \mathrm{a}$ \\
\hline
\end{tabular}

unmodified BM (standardized)

$$
k_{0}=0
$$

\begin{tabular}{r|rrrr}
$\mathrm{N} \backslash \mathrm{T}$ & 60 & 110 & 210 & 250 \\
\hline 50 & 12.5 & 0.0 & 0.0 & 0.0
\end{tabular}

$100 \mathrm{n} / \mathrm{a} \quad 15.4 \quad 0.0 \quad 0.0$

$200 \quad \mathrm{n} / \mathrm{a} \quad \mathrm{n} / \mathrm{a} \quad 12.3 \quad 0.0$

$500 \mathrm{n} / \mathrm{a} \quad \mathrm{n} / \mathrm{a} \quad \mathrm{n} / \mathrm{a} \quad \mathrm{n} / \mathrm{a}$

$k_{0}=1$

\begin{tabular}{r|rrrr}
$\mathrm{N} \backslash \mathrm{T}$ & 60 & 110 & 210 & 250 \\
\hline 50 & 19.1 & 2.9 & 0.5 & 0.3
\end{tabular}

$100 \mathrm{n} / \mathrm{a} \quad 26.0 \quad 0.0 \quad 0.0$

$200 \mathrm{n} / \mathrm{a} \quad \mathrm{n} / \mathrm{a} \quad 30.0 \quad 0.0$

$500 \mathrm{n} / \mathrm{a} \quad \mathrm{n} / \mathrm{a} \quad \mathrm{n} / \mathrm{a} \quad \mathrm{n} / \mathrm{a}$

\begin{tabular}{r|rrrr} 
& \multicolumn{4}{|c}{$k_{0}=2$} \\
$\mathrm{~N} \backslash \mathrm{T}$ & 60 & 110 & 210 & 250 \\
\hline 50 & 23.7 & 10.0 & 5.3 & 4.7 \\
100 & $\mathrm{n} / \mathrm{a}$ & 37.6 & 0.7 & 0.3 \\
200 & $\mathrm{n} / \mathrm{a}$ & $\mathrm{n} / \mathrm{a}$ & 49.1 & 0.4 \\
500 & $\mathrm{n} / \mathrm{a}$ & $\mathrm{n} / \mathrm{a}$ & $\mathrm{n} / \mathrm{a}$ & $\mathrm{n} / \mathrm{a}$
\end{tabular}

unmodified BM (standardized)




Table S5.4: Empirical frequency of correctly identifying only the true weakly dominant (influential) units $\left(m_{0}>0\right.$, and $\left.\alpha=1\right)$

Part A: $m_{0}=1$

unmodifed BM

$$
k_{0}=0
$$

\begin{tabular}{r|rrrr}
$\mathrm{N} \backslash \mathrm{T}$ & 60 & 110 & 210 & 250 \\
\hline 50 & 15.8 & 63.2 & 87.6 & 90.4 \\
100 & $\mathrm{n} / \mathrm{a}$ & 30.1 & 99.0 & 100.0 \\
200 & $\mathrm{n} / \mathrm{a}$ & $\mathrm{n} / \mathrm{a}$ & 44.8 & 99.3 \\
500 & $\mathrm{n} / \mathrm{a}$ & $\mathrm{n} / \mathrm{a}$ & $\mathrm{n} / \mathrm{a}$ & $\mathrm{n} / \mathrm{a}$ \\
& $m_{0}=1, k=1$
\end{tabular}

\begin{tabular}{r|rrrr}
$\mathrm{N} \backslash \mathrm{T}$ & 60 & 110 & 210 & 250 \\
\hline 50 & 13.3 & 59.8 & 87.1 & 89.3
\end{tabular}

$100 \mathrm{n} / \mathrm{a} \quad 27.5 \quad 98.8 \quad 99.6$

$200 \quad \mathrm{n} / \mathrm{a} \quad \mathrm{n} / \mathrm{a} \quad 44.3 \quad 98.7$

$500 \mathrm{n} / \mathrm{a} \quad \mathrm{n} / \mathrm{a} \quad \mathrm{n} / \mathrm{a} \quad \mathrm{n} / \mathrm{a}$

$$
m_{0}=1, k=2
$$

\begin{tabular}{r|rrrr}
$\mathrm{N} \backslash \mathrm{T}$ & 60 & 110 & 210 & 250 \\
\hline 50 & 13.3 & 59.0 & 84.6 & 88.7 \\
100 & $\mathrm{n} / \mathrm{a}$ & 25.6 & 98.8 & 99.5 \\
200 & $\mathrm{n} / \mathrm{a}$ & $\mathrm{n} / \mathrm{a}$ & 44.6 & 98.6 \\
500 & $\mathrm{n} / \mathrm{a}$ & $\mathrm{n} / \mathrm{a}$ & $\mathrm{n} / \mathrm{a}$ & $\mathrm{n} / \mathrm{a}$
\end{tabular}

Part B: $m_{0}=2$

unmodifed BM

$$
k_{0}=0
$$

\begin{tabular}{r|rrrr}
$\mathrm{N} \backslash \mathrm{T}$ & 60 & 110 & 210 & 250 \\
\hline 50 & 4.6 & 43.8 & 74.9 & 79.4 \\
100 & $\mathrm{n} / \mathrm{a}$ & 10.8 & 94.3 & 98.0 \\
200 & $\mathrm{n} / \mathrm{a}$ & $\mathrm{n} / \mathrm{a}$ & 20.7 & 94.2 \\
500 & $\mathrm{n} / \mathrm{a}$ & $\mathrm{n} / \mathrm{a}$ & $\mathrm{n} / \mathrm{a}$ & $\mathrm{n} / \mathrm{a}$ \\
& $m_{0}=2, k=1$
\end{tabular}

\begin{tabular}{r|rrrr}
$\mathrm{N} \backslash \mathrm{T}$ & 60 & 110 & 210 & 250 \\
\hline 50 & 4.2 & 41.6 & 70.2 & 76.5
\end{tabular}

$100 \quad \mathrm{n} / \mathrm{a} \quad 10.2 \quad 94.3 \quad 96.4$

$200 \mathrm{n} / \mathrm{a} \quad \mathrm{n} / \mathrm{a} \quad 17.1 \quad 92.2$

$500 \mathrm{n} / \mathrm{a} \quad \mathrm{n} / \mathrm{a} \quad \mathrm{n} / \mathrm{a} \quad \mathrm{n} / \mathrm{a}$

$$
m_{0}=2, k=2
$$

\begin{tabular}{r|rrrr}
$\mathrm{N} \backslash \mathrm{T}$ & 60 & 110 & 210 & 250 \\
\hline 50 & 3.2 & 39.1 & 68.8 & 73.5 \\
100 & $\mathrm{n} / \mathrm{a}$ & 9.1 & 92.1 & 95.9 \\
200 & $\mathrm{n} / \mathrm{a}$ & $\mathrm{n} / \mathrm{a}$ & 17.0 & 91.2 \\
500 & $\mathrm{n} / \mathrm{a}$ & $\mathrm{n} / \mathrm{a}$ & $\mathrm{n} / \mathrm{a}$ & $\mathrm{n} / \mathrm{a}$
\end{tabular}

unmodified BM (standardized)

$$
k_{0}=0
$$

\begin{tabular}{r|rrrr}
$\mathrm{N} \backslash \mathrm{T}$ & 60 & 110 & 210 & 250 \\
\hline 50 & 21.9 & 69.3 & 85.3 & 87.8 \\
100 & $\mathrm{n} / \mathrm{a}$ & 37.8 & 98.4 & 99.4 \\
200 & $\mathrm{n} / \mathrm{a}$ & $\mathrm{n} / \mathrm{a}$ & 54.9 & 99.0 \\
500 & $\mathrm{n} / \mathrm{a}$ & $\mathrm{n} / \mathrm{a}$ & $\mathrm{n} / \mathrm{a}$ & $\mathrm{n} / \mathrm{a}$ \\
& \multicolumn{4}{c}{$k_{0}=1$}
\end{tabular}

\begin{tabular}{r|rrrr}
$\mathrm{N} \backslash \mathrm{T}$ & 60 & 110 & 210 & 250 \\
\hline 50 & 9.7 & 31.1 & 45.4 & 45.1 \\
100 & $\mathrm{n} / \mathrm{a}$ & 21.7 & 82.0 & 84.9 \\
200 & $\mathrm{n} / \mathrm{a}$ & $\mathrm{n} / \mathrm{a}$ & 37.5 & 89.8 \\
500 & $\mathrm{n} / \mathrm{a}$ & $\mathrm{n} / \mathrm{a}$ & $\mathrm{n} / \mathrm{a}$ & $\mathrm{n} / \mathrm{a}$ \\
& \multicolumn{4}{c}{$k_{0}=2$}
\end{tabular}

\begin{tabular}{r|rrrr}
$\mathrm{N} \backslash \mathrm{T}$ & 60 & 110 & 210 & 250 \\
\hline 50 & 5.9 & 13.5 & 15.9 & 17.4 \\
100 & $\mathrm{n} / \mathrm{a}$ & 12.9 & 52.8 & 59.7 \\
200 & $\mathrm{n} / \mathrm{a}$ & $\mathrm{n} / \mathrm{a}$ & 24.3 & 75.3 \\
500 & $\mathrm{n} / \mathrm{a}$ & $\mathrm{n} / \mathrm{a}$ & $\mathrm{n} / \mathrm{a}$ & $\mathrm{n} / \mathrm{a}$
\end{tabular}

unmodified BM (standardized)

$$
k_{0}=0
$$

\begin{tabular}{r|rrrr}
$\mathrm{N} \backslash \mathrm{T}$ & 60 & 110 & 210 & 250 \\
\hline 50 & 1.4 & 4.9 & 9.0 & 11.5
\end{tabular}

$100 \mathrm{n} / \mathrm{a} \quad 3.1 \quad 43.7 \quad 50.9$

$200 \mathrm{n} / \mathrm{a} \quad \mathrm{n} / \mathrm{a} \quad 10.3 \quad 64.5$

$500 \mathrm{n} / \mathrm{a} \quad \mathrm{n} / \mathrm{a} \quad \mathrm{n} / \mathrm{a} \quad \mathrm{n} / \mathrm{a}$

$k_{0}=1$

\begin{tabular}{r|rrrr}
$\mathrm{N} \backslash \mathrm{T}$ & 60 & 110 & 210 & 250 \\
\hline 50 & 0.2 & 0.6 & 0.7 & 0.7
\end{tabular}

$100 \quad \mathrm{n} / \mathrm{a} \quad 1.3 \quad 11.9 \quad 11.8$

$200 \quad \mathrm{n} / \mathrm{a} \quad \mathrm{n} / \mathrm{a} \quad 3.3 \quad 29.3$

$500 \mathrm{n} / \mathrm{a} \quad \mathrm{n} / \mathrm{a} \quad \mathrm{n} / \mathrm{a} \quad \mathrm{n} / \mathrm{a}$

$k_{0}=2$

\begin{tabular}{r|rrrr}
$\mathrm{N} \backslash \mathrm{T}$ & 60 & 110 & 210 & 250 \\
\hline 50 & 0.0 & 0.1 & 0.0 & 0.0 \\
100 & $\mathrm{n} / \mathrm{a}$ & 0.4 & 1.5 & 1.4 \\
200 & $\mathrm{n} / \mathrm{a}$ & $\mathrm{n} / \mathrm{a}$ & 1.4 & 9.4 \\
500 & $\mathrm{n} / \mathrm{a}$ & $\mathrm{n} / \mathrm{a}$ & $\mathrm{n} / \mathrm{a}$ & $\mathrm{n} / \mathrm{a}$
\end{tabular}


Table S5.5: Average number of non-dominant units falsely selected as dominant $\left(m_{0}>0\right.$, and $\alpha=0.8)$

Part A: $m_{0}=1$

unmodifed BM

$$
k_{0}=0
$$

\begin{tabular}{r|rrrr}
$\mathrm{N} \backslash \mathrm{T}$ & 60 & 110 & 210 & 250 \\
\hline 50 & 25.1 & 10.1 & 4.4 & 3.3 \\
100 & $\mathrm{n} / \mathrm{a}$ & 43.5 & 0.3 & 0.0 \\
200 & $\mathrm{n} / \mathrm{a}$ & $\mathrm{n} / \mathrm{a}$ & 72.9 & 0.6 \\
500 & $\mathrm{n} / \mathrm{a}$ & $\mathrm{n} / \mathrm{a}$ & $\mathrm{n} / \mathrm{a}$ & $\mathrm{n} / \mathrm{a}$ \\
& & $k_{0}=1$ &
\end{tabular}

\begin{tabular}{r|rrrr}
$\mathrm{N} \backslash \mathrm{T}$ & 60 & 110 & 210 & 250 \\
\hline 50 & 26.8 & 12.0 & 4.3 & 3.5
\end{tabular}

$100 \mathrm{n} / \mathrm{a} \quad 44.1 \quad 0.4 \quad 0.3$

$200 \quad \mathrm{n} / \mathrm{a} \quad \mathrm{n} / \mathrm{a} \quad 72.6 \quad 1.3$

$500 \mathrm{n} / \mathrm{a} \quad \mathrm{n} / \mathrm{a} \quad \mathrm{n} / \mathrm{a} \quad \mathrm{n} / \mathrm{a}$

\begin{tabular}{r|rrrr} 
& \multicolumn{4}{|c}{$k_{0}=2$} \\
$\mathrm{~N} \backslash \mathrm{T}$ & 60 & 110 & 210 & 250 \\
\hline 50 & 25.5 & 12.2 & 5.2 & 3.9 \\
100 & $\mathrm{n} / \mathrm{a}$ & 46.8 & 0.7 & 0.3 \\
200 & $\mathrm{n} / \mathrm{a}$ & $\mathrm{n} / \mathrm{a}$ & 69.4 & 1.5 \\
500 & $\mathrm{n} / \mathrm{a}$ & $\mathrm{n} / \mathrm{a}$ & $\mathrm{n} / \mathrm{a}$ & $\mathrm{n} / \mathrm{a}$
\end{tabular}

Part B: $m_{0}=2$

\begin{tabular}{|c|c|c|c|c|c|c|c|c|c|}
\hline \multicolumn{5}{|c|}{$\begin{array}{l}\text { unmodifed BM } \\
k_{0}=0\end{array}$} & \multicolumn{5}{|c|}{$\begin{array}{c}\text { unmodified BM (standardized) } \\
k_{0}=0\end{array}$} \\
\hline $\mathrm{N} \backslash \mathrm{T}$ & 60 & 110 & 210 & 250 & $\mathrm{~N} \backslash \mathrm{T}$ & 60 & 110 & 210 & 250 \\
\hline 50 & 22.5 & 7.1 & 2.7 & 2.2 & 50 & 18.6 & 5.2 & 3.3 & 3.2 \\
\hline 100 & $\mathrm{n} / \mathrm{a}$ & 33.7 & 0.2 & 0.1 & 100 & $\mathrm{n} / \mathrm{a}$ & 29.8 & 0.9 & 0.6 \\
\hline 200 & $\mathrm{n} / \mathrm{a}$ & $\mathrm{n} / \mathrm{a}$ & 48.2 & 0.4 & 200 & $\mathrm{n} / \mathrm{a}$ & $\mathrm{n} / \mathrm{a}$ & 43.0 & 0.9 \\
\hline 500 & $\mathrm{n} / \mathrm{a}$ & $\begin{array}{r}\mathrm{n} / \mathrm{a} \\
k_{0}\end{array}$ & $\begin{aligned} & \mathrm{n} / \mathrm{a} \\
= & 1\end{aligned}$ & $\mathrm{n} / \mathrm{a}$ & 500 & $\mathrm{n} / \mathrm{a}$ & $\begin{array}{r}\mathrm{n} / \mathrm{a} \\
k_{0}\end{array}$ & $\begin{aligned} & \mathrm{n} / \mathrm{a} \\
= & 1\end{aligned}$ & $\mathrm{n} / \mathrm{a}$ \\
\hline $\mathrm{N} \backslash \mathrm{T}$ & 60 & 110 & 210 & 250 & $\mathrm{~N} \backslash \mathrm{T}$ & 60 & 110 & 210 & 250 \\
\hline 50 & 22.1 & 8.5 & 3.3 & 2.5 & 50 & 20.2 & 9.5 & 6.5 & 6.6 \\
\hline 100 & $\mathrm{n} / \mathrm{a}$ & 35.4 & 0.4 & 0.1 & 100 & $\mathrm{n} / \mathrm{a}$ & 35.5 & 4.6 & 3.5 \\
\hline 200 & $\mathrm{n} / \mathrm{a}$ & $\mathrm{n} / \mathrm{a}$ & 52.3 & 0.7 & 200 & $\mathrm{n} / \mathrm{a}$ & $\mathrm{n} / \mathrm{a}$ & 56.8 & 5.8 \\
\hline 500 & $\mathrm{n} / \mathrm{a}$ & $\mathrm{n} / \mathrm{a}$ & $\begin{array}{l}\mathrm{n} / \mathrm{a} \\
2\end{array}$ & $\mathrm{n} / \mathrm{a}$ & 500 & $\mathrm{n} / \mathrm{a}$ & $\begin{array}{r}\mathrm{n} / \mathrm{a} \\
k_{0}\end{array}$ & $\begin{aligned} & \mathrm{n} / \mathrm{a} \\
= & 2\end{aligned}$ & $\mathrm{n} / \mathrm{a}$ \\
\hline $\mathrm{N} \backslash \mathrm{T}$ & 60 & 110 & 210 & 250 & $\mathrm{~N} \backslash \mathrm{T}$ & 60 & 110 & 210 & 250 \\
\hline 50 & 23.2 & 7.9 & 3.5 & 2.9 & 50 & 21.6 & 12.8 & 10.4 & 10.0 \\
\hline 100 & $\mathrm{n} / \mathrm{a}$ & 36.9 & 0.6 & 0.1 & 100 & $\mathrm{n} / \mathrm{a}$ & 41.3 & 12.6 & 10.2 \\
\hline 200 & $\mathrm{n} / \mathrm{a}$ & $\mathrm{n} / \mathrm{a}$ & 56.3 & 0.7 & 200 & $\mathrm{n} / \mathrm{a}$ & $\mathrm{n} / \mathrm{a}$ & 75.1 & 18.5 \\
\hline 500 & $\mathrm{n} / \mathrm{a}$ & $\mathrm{n} / \mathrm{a}$ & $\mathrm{n} / \mathrm{a}$ & $\mathrm{n} / \mathrm{a}$ & 500 & $\mathrm{n} / \mathrm{a}$ & $\mathrm{n} / \mathrm{a}$ & $\mathrm{n} / \mathrm{a}$ & $\mathrm{n} / \mathrm{a}$ \\
\hline
\end{tabular}
$k_{0}=0$

\begin{tabular}{r|rrrr}
$\mathrm{N} \backslash \mathrm{T}$ & 60 & 110 & 210 & 250 \\
\hline 50 & 19.2 & 2.7 & 0.3 & 0.2 \\
100 & $\mathrm{n} / \mathrm{a}$ & 32.7 & 0.0 & 0.0 \\
200 & $\mathrm{n} / \mathrm{a}$ & $\mathrm{n} / \mathrm{a}$ & 46.5 & 0.1 \\
500 & $\mathrm{n} / \mathrm{a}$ & $\mathrm{n} / \mathrm{a}$ & $\mathrm{n} / \mathrm{a}$ & $\mathrm{n} / \mathrm{a}$ \\
& \multicolumn{4}{c}{$k_{0}=1$}
\end{tabular}

\begin{tabular}{r|rrrr}
$\mathrm{N} \backslash \mathrm{T}$ & 60 & 110 & 210 & 250 \\
\hline 50 & 21.2 & 7.3 & 3.3 & 2.8
\end{tabular}

$100 \quad \mathrm{n} / \mathrm{a} \quad 38.7 \quad 1.1 \quad 0.4$

$200 \mathrm{n} / \mathrm{a} \quad \mathrm{n} / \mathrm{a} \quad 59.4 \quad 2.2$

$500 \mathrm{n} / \mathrm{a} \quad \mathrm{n} / \mathrm{a} \quad \mathrm{n} / \mathrm{a} \quad \mathrm{n} / \mathrm{a}$

\begin{tabular}{r|rrrr} 
& \multicolumn{4}{|c}{$k_{0}=2$} \\
$\mathrm{~N} \backslash \mathrm{T}$ & 60 & 110 & 210 & 250 \\
\hline 50 & 23.1 & 11.7 & 8.8 & 8.1 \\
100 & $\mathrm{n} / \mathrm{a}$ & 43.6 & 7.0 & 4.4 \\
200 & $\mathrm{n} / \mathrm{a}$ & $\mathrm{n} / \mathrm{a}$ & 72.1 & 8.6 \\
500 & $\mathrm{n} / \mathrm{a}$ & $\mathrm{n} / \mathrm{a}$ & $\mathrm{n} / \mathrm{a}$ & $\mathrm{n} / \mathrm{a}$
\end{tabular}

unmodified BM (standardized)

Notes: See the notes to Table S5.1. 
Table S6.1: Dominant units in sector-wise industrial production in the U.S.

\begin{tabular}{lcc} 
Approach: & unmodified BM & unmodified BM (standardized) \\
\hline Number of domi- & 137 & 2 \\
nant units: & & \\
Identities: & all except & Automobiles and Light Duty Motor Vehicles \\
& Iron Ore Mining & Motor Vehicle Parts \\
\hline Notes: Data taken from Foerster, Sarte, and Watson (2011). &
\end{tabular}

Table S6.2: Dominant countries in terms of quarterly macroeconomic indicators

\begin{tabular}{|c|c|c|c|c|}
\hline \multirow{2}{*}{$\begin{array}{l}\text { Approach: } \\
\text { Variable: }\end{array}$} & \multicolumn{4}{|c|}{ unmodified BM } \\
\hline & $\log$ real GDP & log real equity prices & real GDP growth & real equity price growth \\
\hline $\begin{array}{l}\text { Number of domi- } \\
\text { nant units: }\end{array}$ & 31 & 25 & 2 & 25 \\
\hline Identities: & $\begin{array}{l}\text { all except } \\
\text { Peru } \\
\text { Turkey }\end{array}$ & $\begin{array}{l}\text { all except } \\
\text { Argentina }\end{array}$ & $\begin{array}{l}\text { France } \\
\text { Spain }\end{array}$ & $\begin{array}{l}\text { all except } \\
\text { Argentina }\end{array}$ \\
\hline Approach: & \multicolumn{4}{|c|}{ unmodified BM (standardized) } \\
\hline Variable: & $\overline{l o g}$ real GDP & log real equity prices & real GDP growth & real equity price growth \\
\hline $\begin{array}{l}\text { Number of domi- } \\
\text { nant units: }\end{array}$ & 27 & 24 & 11 & 1 \\
\hline Identities: & $\begin{array}{c}\text { all except } \\
\text { Brazil } \\
\text { Japan } \\
\text { Peru } \\
\text { Saudi Arabia } \\
\text { Turkey }\end{array}$ & $\begin{array}{c}\text { all except } \\
\text { Argentina } \\
\text { New Zealand }\end{array}$ & * & Netherlands \\
\hline
\end{tabular}

\section{S6 Empirical results for unmodified BM}

In this section we provide results obtained if the unmodified BM procedure is used in our empirical applications. The data sources and transformations are as described in Section 7 of the paper. Again, unmodified BM method is applied to the data with and without standardization. The results are summarized in Tables S6.1-S6.3, and suggest that unmodified BM grossly overestimates the number of dominant units in almost all applications, regularly detecting all but one or two cross section units as dominant. The use of standardized data leads in all but one case to a lower detected number of dominant. However, while the reduction can be quite substantial, in a number of applications the number of dominant units detected using standardized data turn out to be quite large (6 or more in many of the applications). 
Table S6.3: Estimated U.S. states with dominant housing market

\begin{tabular}{lcrl} 
Approach: & unmodified BM & unmodified BM (standardized) \\
\hline Number of domi- & 47 & 6 & \\
nant units: & & & \\
Identities: & all except & Connecticut & Maryland \\
& Nevada & New Hampshire & Virginia \\
& & Massachusetts & Rhode Island \\
\hline Notes: Data taken from Yang (2018) and Freddie Mac House Price Indexes. &
\end{tabular}

